\title{
Epigenetic Regulation of Replication-Dependent Histone mRNA 3' End Processing
}

\section{Dissertation}

\author{
for the award of the degree \\ "Doctor rerum naturalium (Dr. rer. nat.)" \\ Division of Mathematics and Natural Sciences \\ of the Georg-August-Universität Göttingen
}

submitted by

Judith Pirngruber

born in

Oldenburg, Germany

Göttingen, 2010 
Thesis Supervisor:

Prof. Dr. Steven A. Johnsen

Doctoral Committee:

Prof. Dr. Steven A. Johnsen

( ${ }^{\text {st }}$ Referee $)$

Molecular Oncology,

University of Göttingen Medical School, Göttingen

Prof. Dr. Heidi Hahn

Molecular Developmental Genetics,

$\left(2^{\text {nd }}\right.$ Referee $)$

University of Göttingen Medical School, Göttingen

Prof. Dr. Holger Reichardt

Cellular and Molecular Immunology,

University of Göttingen Medical School, Göttingen

Date of the oral examination: $28^{\text {th }}$ April 2010 


\section{Affidavit}

I hereby declare that the $\mathrm{PhD}$ thesis entitled "Epigenetic Regulation of ReplicationDependent Histone mRNA 3' End Processing" has been written independently and with no other sources and aids than quoted.

Judith Pirngruber

March, 2010

Göttingen, Germany 
Für Opa Bruno

„Opa, ich denk' an Dich, wenn ich das Gänseliesel hochkletter'. “ 


\section{Table of contents}

List of Abbreviations ........................................................................................

List of Figures .................................................................................................................... V

List of Tables ..................................................................................................... VII

Abstract..............................................................................................

1 General Introduction .................................................................................

1.1 RNA Polymerase II-dependent transcription and mRNA processing ....... 1

1.1.1 Regulation of transcription by promoter-proximal pausing ................. 1

1.1.2 The positive transcription elongation factor $\mathrm{b}(\mathrm{P}-\mathrm{TEFb})$...................... 3

1.1.3 The "CTD-code" of RNAPII ................................................................ 4

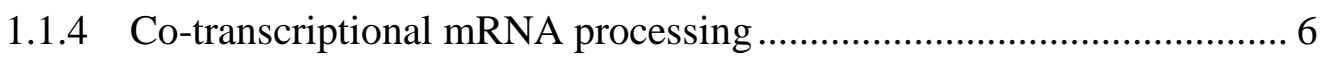

1.1.4.1 Mammalian 3' end processing of polyadenylated mRNA ..... 8

1.1.4.2 The importance of 3 ' end processing .................................... 9

1.2 Chromatin structure and histone modifications ........................................... 9

1.2.1 Histone modifications and the "histone code" .................................. 10

1.2.1.1 Histone H2B monoubiquitination (H2Bub1) ....................... 11

1.2.1.2 Histone $\mathrm{H} 3$ lysine 4 trimethylation (H3K4me3) .................. 13

1.2.1.3 Histone $\mathrm{H} 3$ lysine 36 trimethylation (H3K36me3)............. 14

1.2.1.4 Histone modification crosstalk.......................................... 15

1.2.2 Movement through chromatin is coupled

to posttranslational histone modifications.......................................... 16

1.2.3 Poised promoters show hallmarks of transcription initiation.............. 17

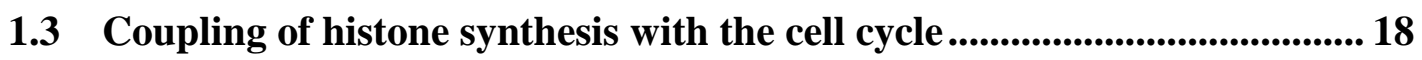

1.3.1 Proteins that regulate the progression of the cell cycle...................... 18

1.3.1.1 Cyclins and cyclin-dependent kinases (CDKs) .................... 19

1.3.1.2 The retinoblastoma protein $(\mathrm{pRB})$.................................. 21

1.3.1.3 The E2F transcription factor family members .................... 21

1.3.2 Histone synthesis is strictly coupled to the cell cycle ........................ 23

1.3.2.1 Replication-dependent histone genes: organization and localization ............................................. 23

1.3.2.2 Replication-dependent histone mRNA 3' end processing ... 24

1.3.2.3 Transport, translation and degradation of histone mRNA ... 26

1.3.2.4 Regulation of replication-dependent histone gene transcription ....

1.3.2.5 The existence of polyadenylated replication-dependent histone mRNAs

1.3.3 The tumor suppressor $\mathrm{p} 53$ regulates

cell cycle progression through $\mathrm{p} 21$

1.3.3.1 The p53 regulator MDM2 and its inhibition by Nutlin-3a... 30

1.3.3.2 Does p53 regulation play a role in the expression of polyadenylated replication-dependent histone mRNAs? 


\subsection{Publication I:}

CDK9 directs $\mathrm{H} 2 \mathrm{~B}$ monoubiquitination and controls

replication-dependent histone mRNA 3' end processing

2.1.1 Supplementary Material 46

\subsection{Publication II:}

Insights into the function of the human $\mathrm{P}-\mathrm{TEFb}$ component CDK9 in the regulation of chromatin modifications and co-transcriptional mRNA processing

\subsection{Publication III:}

Induced $\mathrm{G}_{1}$ cell cycle arrest controls replication-dependent

histone mRNA 3' end processing through p21, NPAT and CDK9

2.3.1 Supplementary Material

3 General Discussion

3.1 Chromatin structure, histone modifications and the CTD of RNAPII....95

3.1.1 A CTD-independent way for monoubiquitination of human histone $\mathrm{H} 2 \mathrm{~B}$ ?

3.1.2 The role of H2Bub1 in chromatin assembly and disassembly ............ 98

3.1.3 Chromatin structure versus CTD-code:

What gives the signal for productive transcription? ........................ 100

3.1.4 A role for pausing in mRNA 3' end formation .............................. 101

\subsection{Polyadenylated replication-dependent histone transcripts:}

formation and possible functions.

3.2.1 Is polyadenylation of replication-dependent histone transcripts a result of splicing?...

3.2.2 Function and regulation of NPAT in replication-dependent histone mRNA 3' end processing

3.2.3 $\mathrm{G}_{0} / \mathrm{G}_{1}$ cell cycle arrest but not a $\mathrm{G}_{2}$ arrest seems to be relevant for the production of polyadenylated histone mRNAs

3.2.4 The diverse mechanisms by which CDK9 controls histone mRNA 3' end processing

3.2.5 What is the molecular mechanism which controls the mode of replication-dependent histone mRNA 3' end processing?

3.2.6 Possible physiological functions of polyadenylated replication-dependent histone mRNA transcripts

4 Summary and Conclusions

5 Reference List

6 Acknowledgements. 


\section{List of Abbreviations}

\begin{tabular}{|c|c|}
\hline$\left(\mathrm{NH}_{4}\right)_{2} \mathrm{SO}_{4}$ & ammonium sulphate \\
\hline $36 B 4$ & mouse homolog of human $R P L P O$ gene \\
\hline $5-\mathrm{FU}$ & 5-Fluorouracil \\
\hline 7-AAD & 7-amino-actinomycin D \\
\hline 7SK snRNA & 7SK small nuclear RNA \\
\hline ActD & Actinomycin D \\
\hline ADP & adenosine diphosphate \\
\hline $\mathrm{APC} / \mathrm{C}$ & anaphase-promoting complex/cyclosome \\
\hline Asf1 & anti-silencing factor 1 \\
\hline ASH2L & absent, small, or homeotic-like protein \\
\hline ATM & ataxia telangiectasia mutated \\
\hline ATP & adenosine triphosphate \\
\hline BGP & beta-glycerophosphate \\
\hline $\mathrm{bp}$ & base pair \\
\hline BRD4 & bromodomain containing 4 protein \\
\hline BrdU & 5-bromodeoxyuridine \\
\hline Bre1p & yeast brefeldin A sensitive protein 1 \\
\hline Bur1, 2 & bypass upstream activating sequence requirement protein 1,2 \\
\hline CAK & cyclin-dependent kinase activating kinase \\
\hline $\mathrm{CB}$ & cajal body \\
\hline CBC & cap binding complex \\
\hline CBP80 & cap binding protein 80 \\
\hline CCND1 & cyclin D1 \\
\hline $\mathrm{CDC}$ & cell division cycle \\
\hline CDK & cyclin-dependent kinase \\
\hline cDNA & complementary DNA \\
\hline $\mathrm{CE}$ & capping enzyme \\
\hline $\mathrm{CF}$ & cleavage factor \\
\hline CHD1 & chromodomain protein 1 \\
\hline ChIP & cromatin immunoprecipitation \\
\hline Cip & cyclin inhibitor protein \\
\hline CKI & cyclin-dependent kinase inhibitor \\
\hline COMPASS & complex of proteins associated with Set1 \\
\hline Cont. & control \\
\hline CPSF & cleavage and polyadenylation specifity factor \\
\hline CSTF & cleavage stimulating factor \\
\hline CTD & carboxy-terminal domain \\
\hline Ctr9p & yeast cyclin three requiring 9 protein \\
\hline CUL1 & cullin 1 \\
\hline DCP1, 2 & decapping protein 1,2 \\
\hline DHFR & dihydrofolate reductase \\
\hline DISC & death-inducing signaling complex \\
\hline dMes-4 & Drosophila maternal effect sterile family member 4 \\
\hline DMSO & dimethyl sulfoxide \\
\hline DNA & deoxyribonuleic acid \\
\hline DOT1L & disruptor of telomeric silencing 1-like protein \\
\hline $\mathrm{DP} 1,2$ & dimerization partner 1,2 \\
\hline DRB & 5,6-dichloro-1- $\beta$-D-ribofuranosyl-benzimidazole \\
\hline DSE & downstream sequence element \\
\hline DSIF & DRB sensitivity-inducing factor \\
\hline $\mathrm{E}(\mathrm{z})$ & enhancer of zeste \\
\hline E1 & ubiquitin activating enzyme \\
\hline E2 & ubiquitin conjugating enzyme \\
\hline $\mathrm{E} 2 \mathrm{~F}$ & E2 promoter binding factor \\
\hline E3 & ubiquitin-protein-isopeptide ligase \\
\hline EDTA & ethylenediaminetetraacetic acid \\
\hline EIF4G & eukaryotic translation initiation factor $4-\gamma$ \\
\hline $\mathrm{ER} \alpha$ & estrogen receptor $\alpha$ \\
\hline
\end{tabular}




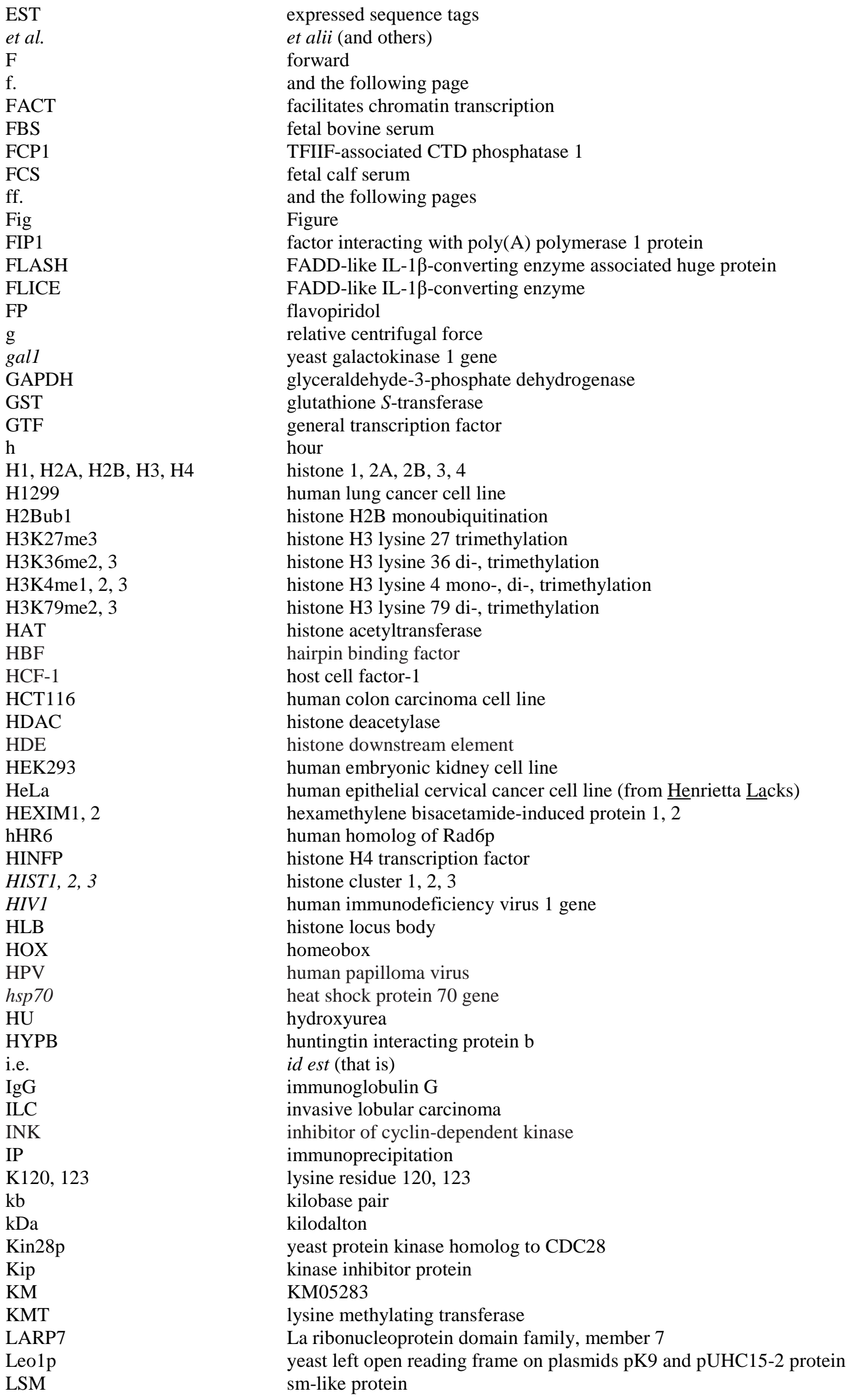




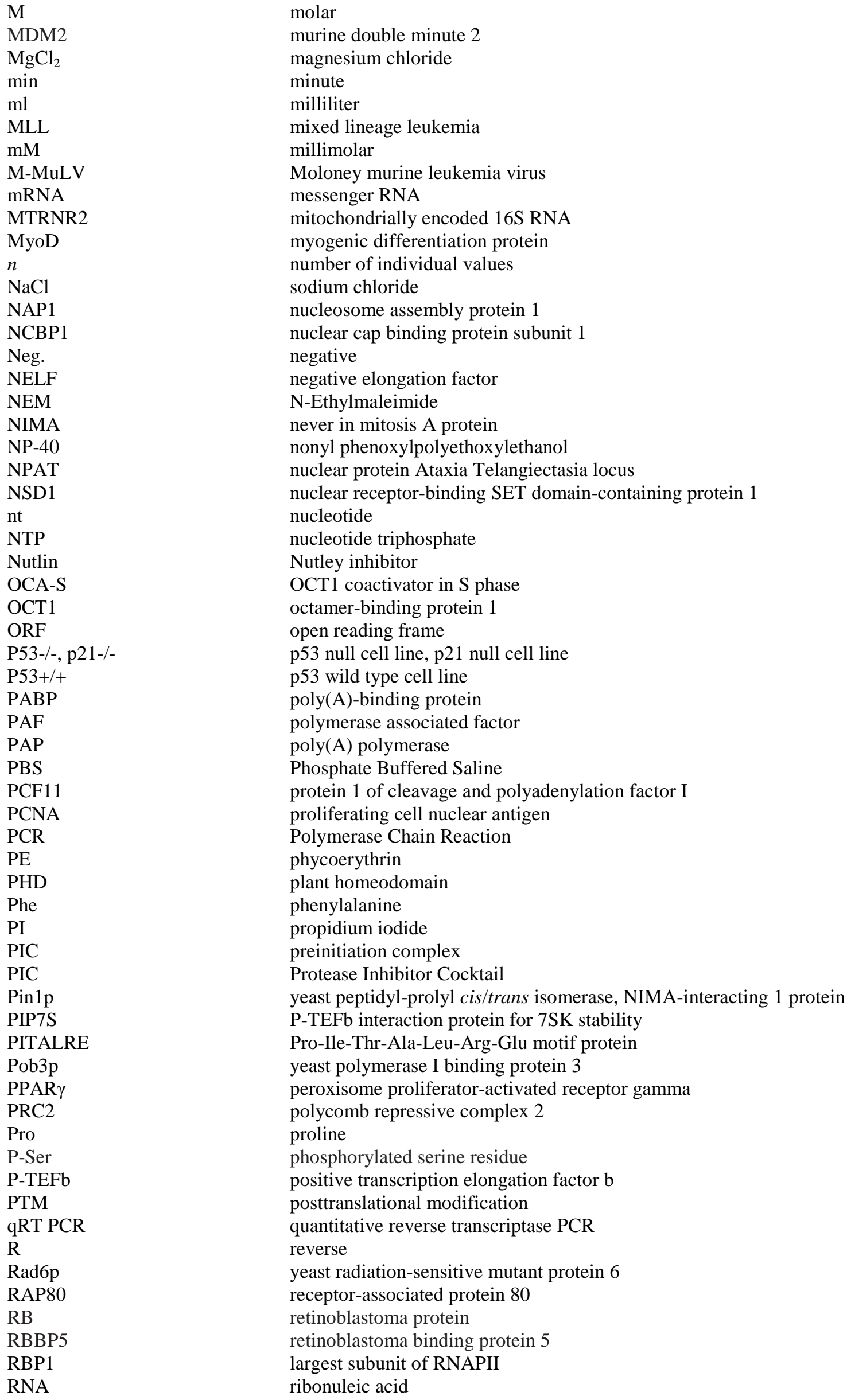




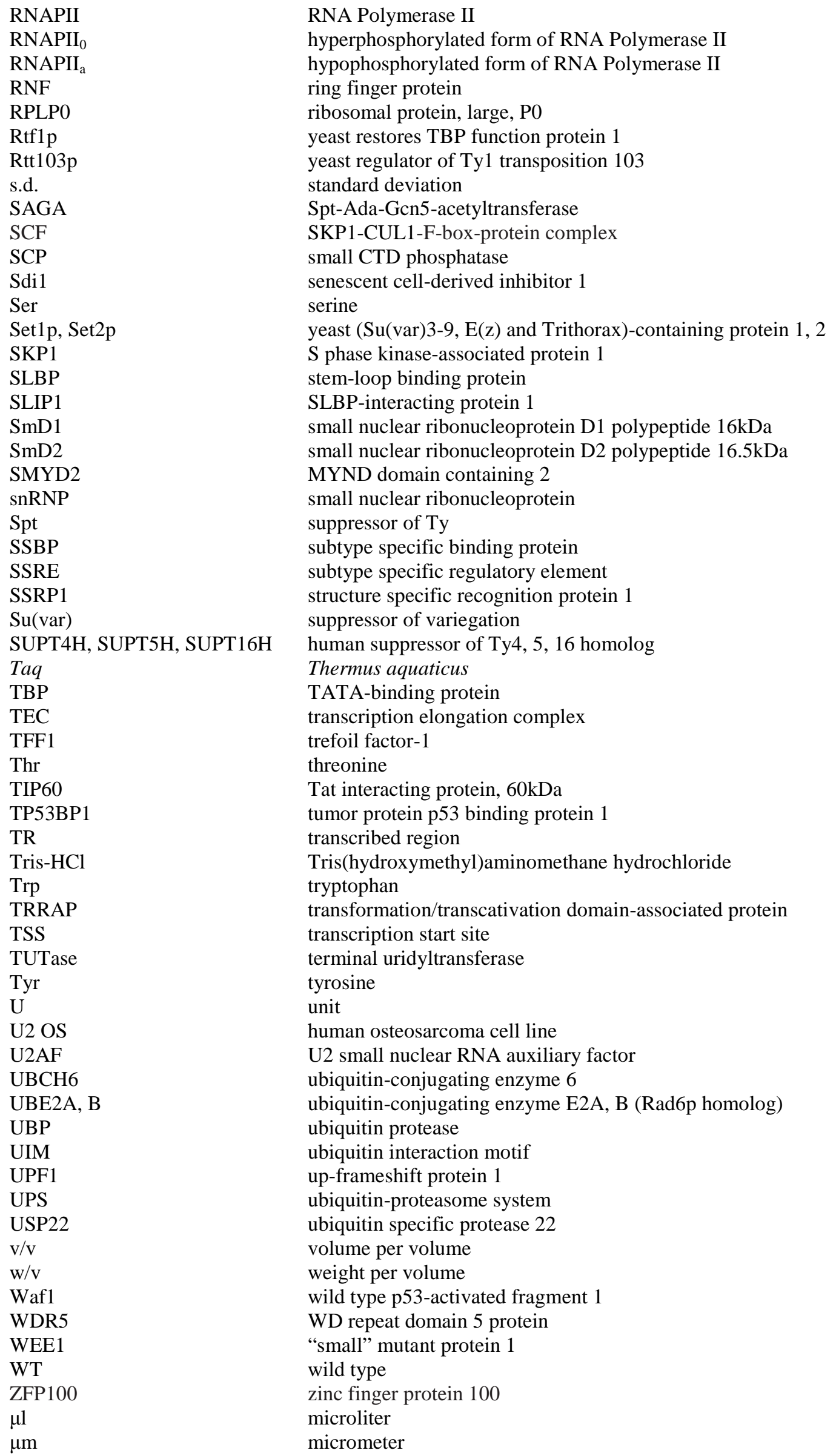




\section{List of Figures}

\section{General Introduction, General Discussion, Summary and Conlusions}

Fig 1

Fig 2

Fig 3

Fig 4

Fig 5

Fig 6

Fig 7

Fig 8

\section{Publication I}

Fig I.1

Fig I.2

Fig I.3

Fig I.4

Supp Fig I.S1

Supp Fig I.S2

\section{Publication II}

Fig II.1

Fig II.1

Fig II.2

\section{Publication III}

Fig III.1

Fig III.2

Fig III.3

Fig III.4

Fig III.5

Fig III.6

Supp Fig III.S1

Supp Fig III.S2

Supp Fig III.S3

Supp Fig III.S4

Supp Fig III.S5
RNAPII-dependent transcription and the CTD phosphorylation.......................... 6

Posttranslational modifications of the core histones and their positions ............... 11

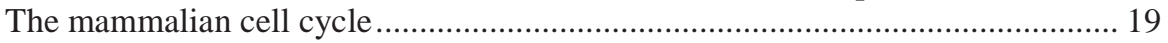

Formation of the processing complex on canonical histone pre-mRNAs............. 26

Model for the function of NPAT in activating histone genes ............................ 28

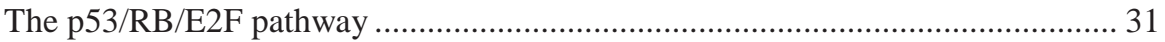

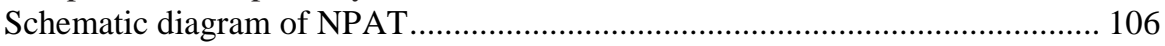

Schematic representation for the role of $\mathrm{G}_{1} / \mathrm{G}_{0}$ arrest in controlling

the mode of replication-dependent histone 3 ' end formation

$116 f$.

H2Bub1 depends on CDK9 activity rather than transcriptional activation per se 37

CDK9 activity is essential for maintaining global levels of H2Bub1 .................... 38

CDK9 and H2Bub1 direct replication-dependent histone

messenger RNA 3'-end processing but not transcription .................................. 41

CDK9 activity recruits proteins involved in $\mathrm{H} 2 \mathrm{~B}$ ubiquitination and histone messenger RNA 3'-end formation, and decreases RNAPII read-through ............. 42 CDK9, RNF20 and RNF40 are essential for maintaining global levels of $\mathrm{H} 2 \mathrm{~B}$ monoubiquitination. CDK9, RNF20/40 and associated proteins and chromatin modifying enzymes specifically affect replication-dependent histone mRNA polyadenylation

CDK9 activity is essential for $\mathrm{H} 3 \mathrm{~K} 4 \mathrm{me} 3$ and $\mathrm{H} 3 \mathrm{~K} 36 \mathrm{me} 3$ 56 CDK9 and RNF20/40 knockdown increase the formation of spliced HIST1H2BD (A) and HIST1H2AC (B) transcripts Schematic representation of the role of CDK9 in controlling replication-dependent histone mRNA 3' end processing.

P53 accumulation reciprocally regulates replication-dependent histone gene expression and mRNA polyadenylation in a p21-dependent manner ........... 70 Accumulation of p53 decreases the recruitment of CDK9, PAF1 and NPAT ....... 72 Accumulation of $\mathrm{p} 53$ and induction of $\mathrm{p} 21$ precede cell cycle arrest, decreases in replication-dependent histone and NPAT gene expression and ultimately accumulation of polyadenylated histone transcripts

Accumulation of polyadenylated replication-dependent histone mRNAs is induced following a $\mathrm{G}_{1} / \mathrm{G}_{0}$ cell-cycle arrest ..................................... 77

NPAT expression is essential for optimal levels of replication-dependent gene transcription and proper pre-mRNA 3' end processing . NPAT knockdown mimics p53-induced $\mathrm{G}_{1}$ cell-cycle arrest and is required for optimal recruitment of CDK9 and PAF1..... Nutlin-3a treatment does not affect cell cycle progression in the absence of $\mathrm{p} 53$ or $\mathrm{p} 21$

Nutlin-3a treatment does not significantly induce apoptosis

in HCT116 cells irrespective of the p53 and p21 status ..................................... 88

Total H2B protein levels do not change following Nutlin-3a treatment................ 88

Nutlin-3a time-dependently decreases the fraction of HCT116 cells in S phase... 89 NPAT downregulation depends upon an intact p53-p21 axis 90 
Supp Fig III.S6

Accumulation of polyadenylated replication-dependent histone mRNAs is induced following a $\mathrm{G}_{1} / \mathrm{G}_{0}$ cell-cycle arrest

Supp Fig III.S7

Supp Fig III.S8

Supp Fig III.S9
The effect of Cyclin E1 on replication-dependent histone gene expression and pre-mRNA 3' end processing is independent of p53 status... NPAT expression is essential for optimal levels of replication-dependent gene transcription and proper mRNA 3' end processing. Decreased CDK9, PAF1 and NPAT recruitment to replication-dependent histone genes is a specific effect of NPAT knockdown and not due to non-specific effects of siRNA transfection. 92 


\section{List of Tables}

\section{$\underline{\text { Publication I }}$}

Supp Table I.S1

Supp Table I.S2

Supp Table I.S3

Supp Table I.S4

\section{$\underline{\text { Publication III }}$}

Supp Table III.S1

Supp Table III.S2

Supp Table III.S3

Supp Table III.S4
Antibodies used for ChIP and western blot analyses and the respective dilutions 48 Primers utilized in ChIP in 5' to 3' orientation ................................................... 49

Primers utilized in RT-PCR in 5' to 3' orientation.............................................. 49

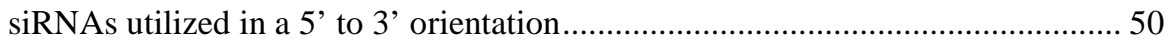

siRNAs utilized for knockdown studies in a 5' to 3' orientation.......................... 85 Antibodies used for ChIP, IP, western blot analyses and Immunofluorescence and the respective dilutions.... $85 f$. Primers utilized for chromatin immunoprecipitation analyses in 5' to 3' orientation 86 Primers utilized for gene expression analyses in 5' to 3' orientation $86 f$. 


\section{Abstract}

Cyclin-dependent kinase 9 (CDK9) was shown to be the Ser/Thr kinase that phosphorylates the C-terminal domain (CTD) of the largest subunit of RNA Polymerase II (RNAPII) at serine residue 2 in mammalian thus regulating its activity following transcriptional inititiation. Furthermore, CDK9 was suggested to also regulate cotranscriptional histone modifications and mRNA processing events. Our studies show that CDK9 functions in maintaining global levels of histone H2B lysine 120 monoubiquitination (H2Bub1) at lysine 120 and guides a complex network for additional histone modifications, including histone $\mathrm{H} 3$ lysine 4 trimethylation (H3K4me3) and H3K36me3. However, these modifications seem to not only be dependent upon phosphorylation of the CTD but include other CDK9 targets like suppressor of Ty 5 homolog (SUPT5H), negative elongation factor-E (NELF-E) and probably the ubiquitin-conjugating enzyme E2A (UBE2A). Interestingly, we found that CDK9 activity and the mentioned histone modifications are necessary for maintaining proper replication-dependent histone mRNA 3' end processing since CDK9 knockdown resulted in an inefficient recognition of the correct cleavage site and led to read-through of RNAPII to an alternative downstream polyadenylation signal. Furthermore, induction of a $G_{1}$ cell cycle arrest by accumulation of $\mathrm{p} 53$ resulted in an increase in polyadenylated replication-dependent histone mRNA transcripts as well via reduced expression of the E2F-dependent histone-specific transcription regulator nuclear protein Ataxia Telangiectasia locus (NPAT). We could show that NPAT can directly interact with the positive transcription elongation factor $\mathrm{b}(\mathrm{P}-\mathrm{TEFb})$ and thus recruits $\mathrm{CDK} 9$ to replication-dependent histone genes playing a role in proper replication-dependent histone mRNA 3' end processing. These latest results uncover a mechanism of a regulated switch form processed replication-dependent histone mRNA transcripts to polyadenylated ones during a normal cellular process. 


\section{General Introduction}

\subsection{RNA Polymerase II-dependent transcription and mRNA processing}

In eukaryotes protein coding genes are transcribed by RNA Polymerase II (RNAPII) from DNA into messenger RNA (mRNA) in the nucleus. Subsequently, the mRNA is transported into the cytoplasm and translated into proteins. The transcript generation by RNAPII takes place in a so-called transcription cycle that includes the three main phases initiation, elongation, and termination. Before the first step takes place, general transcription factors (GTFs) build a platform at the promoter of the respective gene and recruit RNAPII to form the preinitiation complex (PIC). In detail, the PIC includes RNAPII, the general factors TFIID, IIB, IIE, IIF, IIH and additional cofactors (Orphanides et al., 1996). TFIIH, the last initiation factor that binds to the PIC, has ATPase, helicase and kinase activity (Svejstrup et al., 1996). After formation of an open complex between RNAPII and the melted double-stranded DNA, which is an ATP-dependent process, the first phosphodiester bond is built and the presence of nucleotide triphosphates (NTPs) allows RNAPII to clear the promoter. The kinase activity of TFIIH finally leads to the phoshorylation of the carboxy-terminal domain (CTD) of the largest subunit of RNAPII (RBP1), at serine residue 5 (Ser5) being an important part of transcriptional regulation (Lee and Young, 2000). This event will be described in detail in section 1.1 .3 on page $4 \mathrm{ff}$. In the early stage of elongation the transcription elongation complex (TEC) is instable and has the tendency to release the transcribed mRNA, a process which is called abortive initiation (Sims III et al., 2004). It was shown that after synthesis of a 23 nucleotide long transcript the slippage becomes undetectable and a stabilized TEC is formed (Pal and Luse, 2003). The promoter escape may be followed by an additional regulatory step, promoter-proximal pausing, which may be subsequently followed by productive elongation (Saunders et al., 2006). The transcription cycle finally ends with the termination phase in which the mRNA is cleaved and polyadenylated and RNAPII is removed from the gene (Proudfoot et al., 2002).

\subsubsection{Regulation of transcription by promoter-proximal pausing}

Promoter-proximal pausing, a rate limiting and regulatory step, occurs when the transcript reaches a size of 20-50 bases (Bentley, 2005). This phenomenon was first 
demonstrated for the heat-shock gene hsp70 of Drosophila melanogaster (Gilmour and Lis, 1986). Before RNAPII commits into productive elongation, promoter-proximal pausing functions as a checkpoint and is controlled by specific negative elongation factors that will now be introduced (Saunders et al., 2006). The entry of RNAPII into productive elongation is repressed by two important negative factors the DRB sensitivity-inducing factor (DSIF) consisting of the subunits suppressor of Ty4 (Spt4p) in yeast or suppressor of Ty4 homolog (SUPT4H) in humans, Spt5p or SUPT5H respectively and the negative elongation factor (NELF) which comprises four subunits NELF-A, B, C/D and E (Narita et al., 2003; Wada et al., 1998a; Yamaguchi et al., 1999). DSIF can bind to the hypophosphorylated form of RNAPII (RNAPII ${ }_{a}$ ) and NELF then binds to the preformed binary DSIF/RNAPII complex and the nascent RNA which leads to an increased pausing time and a decreased transcription rate of RNAPII (Renner et al., 2001; Yamaguchi et al., 2002).

It is postulated that the pausing of RNAPII mediated by DSIF and NELF is needed to allow the recruitment of the capping enzyme (CE) and hence the addition of a 7-methyl guanosine cap to the 5' end of the nascent transcript (Sims III et al., 2004). The CE associates with the Ser5-phosphorylated form of the CTD of RNAPII and with SUPT5H (Rodriguez et al., 2000; Wen and Shatkin, 1999). The efficient release of paused RNAPII into the productive elongation phase is largely controlled by the positive transcription elongation factor $\mathrm{b}(\mathrm{P}-\mathrm{TEFb})$. $\mathrm{P}-\mathrm{TEFb}$ phosphorylates the CTD of RNAPII at serine residue 2 (Ser2) as well as the human DSIF subunit SUPT5H. SUPT5H is phosphorylated at a C-terminal repeat similar to the RNAPII-CTD and this modification leads to a conversion from a negative to a positive function of DSIF in transcriptional elongation (Peterlin and Price, 2006; Yamada et al., 2006). In addition, $\mathrm{P}-\mathrm{TEFb}$ phosphorylates NELF-E, thereby releasing RNAPII from the repressive complex (Fujinaga et al., 2004; Peterlin and Price, 2006). Furthermore, the chromatin specific transcription elongation factor FACT (facilitates chromatin transcription) that was originally identified for its role in allowing elongation through chromatin, cooperates with $\mathrm{P}-\mathrm{TEFb}$ to remove the DSIF/NELF-mediated negative regulation of transcription (Wada et al., 2000). The combined effects of different P-TEFb phosphorylation events relieves the repressing action of these negative elongation factors on RNAPII which allows the change from promoter-proximal pausing to productive elongation (Fujinaga et al., 2004; Ivanov et al., 2000; Kim and Sharp, 2001; Renner et al., 2001; Wada et al., 1998b; Yamaguchi et al., 1999). P-TEFb thus has 
separate functions to promote transcription. On the one hand it blocks the activity of repressors in the early stage of elongation and on the other hand it phosphorylates the CTD during productive elongation (Wood and Shilatifard, 2006). The fact that productive elongation requires a variety of additional elongation factors shows that this is a highly regulated process.

\subsubsection{The positive transcription elongation factor $b$ (P-TEFb)}

The positive transcription elongation factor $\mathrm{b}$ was identified as a factor that can stimulate 5,6-dichloro-1- $\beta$-D-ribofuranosyl-benzimidazole (DRB) sensitive transcription and is required for RNAPII to synthesize long transcripts (Marshall and Price, 1992). The complex is a heterodimer composed of the cyclin-dependent kinase 9 (CDK9), a cell division cycle protein 2 (CDC2)-like Serine/Threonine kinase, and one of four cyclin partners, Cyclin T1, T2a, T2b or Cyclin K (Fu et al., 1999; Peng et al., 1998b; Peng et al., 1998a). CDK9 was identified in 1994 and first named PITALRE, based on a conserved sequence found in CDC2 and related kinases, due to its unknown function and interaction partners (Grana et al., 1994). In addition to the well known 42 $\mathrm{kDa}$ form of the CDK9 protein a second, larger form, CDK9(55), was identified that is translated from an mRNA that is generated by using a second upstream promoter of the CDK9 gene (Shore et al., 2003). As a general transcription factor P-TEFb is required for the efficient transcription of most genes. Its main function is the Ser2-phosphorylation of the CTD of RNAPII thereby promoting transcriptional elongation and other cotranscriptional processes (Marshall et al., 1996).

Compared to the functions of P-TEFb in mammals there are two homologs that combine its activities in Saccharomyces cerevisiae which include the Bur1p/Bur2p and the Ctk kinase complexes (consisting of Ctk1p, Ctk2p and Ctk3p) that may both function in the phosphorylation of the CTD (Wood and Shilatifard, 2006).

$\mathrm{P}-\mathrm{TEFb}$ can be found either in an active or in an inactive complex. The core active complex comprises CDK9, Cyclin T1 or T2 and the bromodomain containing 4 protein (BRD4) (Jang et al., 2005). BRD4 was shown to recruit $\mathrm{P}-\mathrm{TEFb}$ to transcriptional templates in vitro and in vivo and since BRD4 binds acetylated residues on histones it may facilitate $\mathrm{P}-\mathrm{TEFb}$ recruitment to active genes (Bres et al., 2008; Wu and Chiang, 2007; Yang et al., 2005).

Besides the core active P-TEFb complex an inactive "large" P-TEFb complex exists and contains at least $50 \%$ of CDK9 in HeLa cells. In addition to CDK9 and 
Cyclin T1 or T2 the large complex comprises the 7SK small nuclear RNA (7SK snRNA) and the hexamethylene bisacetamide-induced protein 1 or 2 (HEXIM1,2) (Michels et al., 2003; Michels et al., 2004; Nguyen et al., 2001; Yang et al., 2001; Yik et al., 2003). It is postulated that a single molecule of 7SK snRNA binds a dimer of HEXIM1 or HEXIM2 thus changing its conformation so that it exposes a binding domain for P-TEFb. Phosphorylation of T186 of the T-loop of CDK9 allows the binding of two complexes to the HEXIM proteins resulting in an inactive P-TEFb complex (Li et al., 2005b; Marshall and Grana, 2006). Another component of the inactive P-TEFb complex is La ribonucleoprotein domain family, member 7 (LARP7) also designated as PIP7S (P-TEFb interaction protein for 7SK stability) (He et al., 2008; Krueger et al., 2008; Markert et al., 2008). As a stable component of the 7SK snRNP it stays bound to 7SK snRNP when P-TEFb is activated and released and its expression is essential for maintaining 7SK stability and the integrity of the large complex (Krueger et al., 2008).

A further possibility of regulating P-TEFb activity is the acetylation of CDK9 with the major acetylation site being at lysine 44. This posttranslational modification was shown to enhance the ability of $\mathrm{P}-\mathrm{TEFb}$ to phosphorylate the serine 2 residue of the CTD of RNAPII (Fu et al., 2007).

\subsubsection{The "CTD-code" of RNAPII}

In addition to its catalytical core, the largest subunit of eukaryotic RNAPII (RBP1) contains an unusual carboxy-terminal domain (CTD) that comprises 52 repeated heptapeptides in humans and mice with the consensus sequence Tyr-Ser-ProThr-Ser-Pro-Ser $\left(\mathrm{Y}_{1} \mathrm{~S}_{2} \mathrm{P}_{3} \mathrm{~T}_{4} \mathrm{~S}_{5} \mathrm{P}_{6} \mathrm{~S}_{7}\right)$ (Corden et al., 1985; Corden, 1990). This sequence is evolutionary conserved but the number of repeats varies from 26 in yeast, 32 in nematodes, 45 in Drosophila melanogaster and 52 in mammals (Egloff and Murphy, 2008). It is a target for phosphorylation which can act as a platform for proteins that are implicated in different co-transcriptional processes (Brookes and Pombo, 2009; Dahmus, 1995).

Two forms of RNAPII either containing a hypo- or a hyperphosphorylated CTD (RNAPII $_{a}$ and RNAPII $_{0}$ respectively) exist (Dahmus, 1981). The non- or hypophosphorylated RNAPII is associated with the PIC at the promoter whereas the hyperphosphorylated form is part of the elongating complex. As already mentioned in section 1.1 on page 1 and section 1.1 .2 on page 3 f. phosphorylation mainly occurs on 
Ser2 and Ser5 of the heptapeptide but was recently found on Ser7 (Chapman et al., 2007; Egloff et al., 2007). Phosphorylation of Thr4 and Tyr1 have also been postulated, as has glycosylation of Thr4. Ser5 phosphorylation occurs near the 5' end of genes and is carried out by the cyclin-dependent kinase 7 (CDK7) a component of the general transcription factor TFHII that binds to the hypophosphorylated form of RNAPII (Komarnitsky et al., 2000). This modification allows the binding of the guanyltransferase that is responsible for addition of the 7-methyl guanosine cap to the 5' end of the newly synthesized mRNA (Cho et al., 1998). When promoter-proximal pausing occurs after promoter escape CDK9 as part of $\mathrm{P}-\mathrm{TEFb}$ phosphorylates the CTD at Ser2 allowing RNAPII for productive elongation (Marshall et al., 1996). 3' end processing factors like the protein 1 of cleavage and polyadenylation factor I (PCF11) were shown to directly bind to P-Ser2 repeats containing CTDs (Meinhart and Cramer, 2004). Chromatin Immunoprecipitation (ChIP) results showed that P-Ser5 of the CTD predominates near the transcription start site of a gene, whereas in the middle of a gene both Ser2 and Ser5 are phosphorylated and near the 3' end Ser2 is extensively phosphorylated (Phatnani and Greenleaf, 2006). Phosphorylation of Ser7 appears to play a gene-specific role being required for the proper expression of snRNA genes but not protein-coding genes. Furthermore, it seems to be critical for recruiting the Integrator complex which is necessary for the 3' end processing of snRNAs (Egloff et al., 2007). Recent findings indicated that the kinase homolog to CDC28 (Kin28p) in yeast and its homolog CDK7 in humans are the kinases responsible for phosphorylation of Ser7 (Akhtar et al., 2009; Boeing et al., 2010).

Since the phosphorylation of the CTD is a dynamic process, also dephosphorylation must be performed by specific phosphatases. The TFIIF-associated CTD phosphatase 1 (FCP1) and the small CTD phosphatase 1 (SCP1) were reported to dephosphorylate Ser2 and Ser5 respectively of the heptapeptide sequence (Egloff and Murphy, 2008; Meinhart et al., 2005; Yeo et al., 2003; Yeo et al., 2005).

Phosphorylated RNAPII is associated with pre-mRNA processing factors like the 5' end capping enzyme, splicing factors, cleavage and polyadenylation factors (Bentley, 2005). Up to now, 8 different phosphorylation patterns within the repeat are possible (unphosphorylated CTD, combinations of P-Ser2, P-Ser5 and P-Ser7 CTD). These different modification patterns appear to play a role in the recruitment of specific regulatory factors that control the activity of transcribing RNAPII (Corden, 2007). Thus, differential posttranslational modifications of the CTD residues within the 
heptapeptide sequence generate a so-called "CTD code" that serves as a platform for a variety of proteins that are timely recruited during the transcription cycle (Egloff and Murphy, 2008). In addition to the phosphorylation pattern the cis/trans isomerization of the proline residues Pro3 and Pro6 by the peptidyl-prolyl cis/trans isomerase, NIMAinteracting 1 protein (Pin1p) which binds to Ser2- and Ser5-phosphorylated CTD and change the structure of the CTD likely also add to the complexity of the "CTD code" (Buratowski, 2003).

Each of these different possible conformation patterns of the CTD is thought to play its own role in the regulation of RNAPII-dependent transcription by being responsible for specific transcriptional steps and co-transcriptional pre-mRNA processing events. Theoretically, every residue is a potential target for some kind of posttranslational modification. Thus, multiple CTD modifications help to recruit specific proteins which play important roles in the regulation of transcription (illustrated in Fig 1 on page 6).

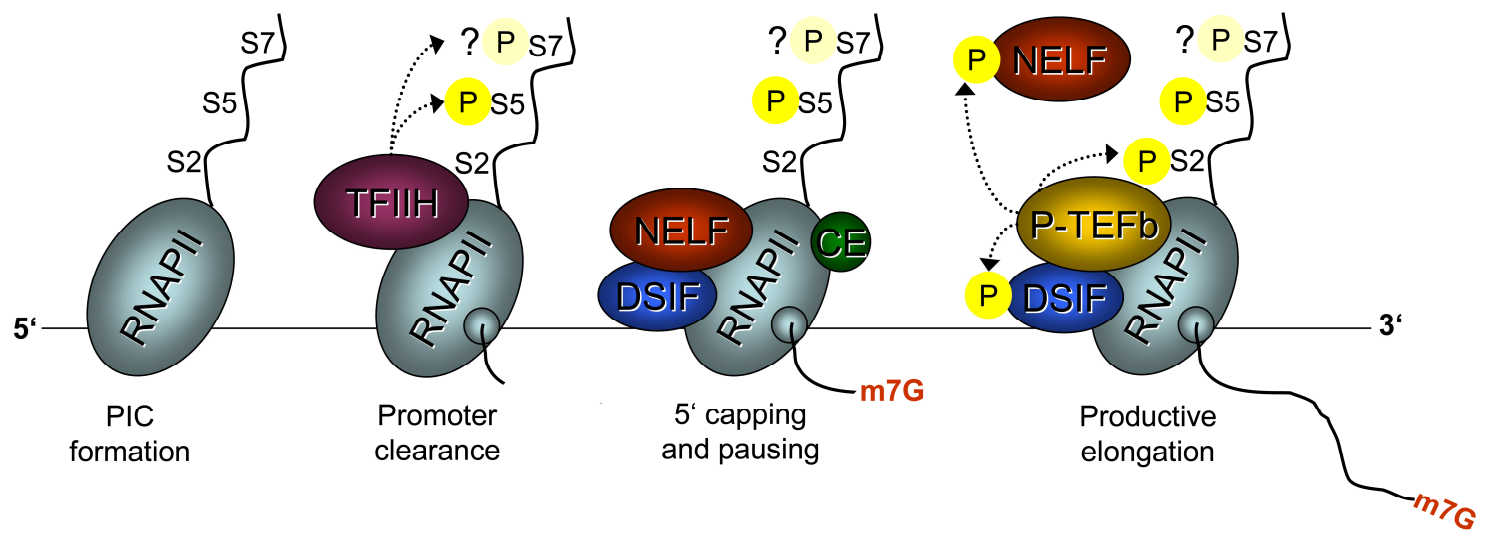

Fig 1: RNAPII-dependent transcription and the CTD phosphorylation patterns. When the PIC is built RNAPII is present in a hypophosphorylated form. TFIIH then phosphorylates Ser5 and in some genes Ser7 which leads to promoter clearance. Pausing takes place upon binding of the negative elongation factors NELF and DSIF, the capping enzyme (CE) is recruited and 5' capping takes place. Upon recruitment of P-TEFb, Ser2, DSIF and NELF are phosphorylated, leading to the dissociation of NELF, the conversion of DSIF into a positive transcription elongation factor and consequently to productive elongation (modified from Peterlin and Price, 2006; Phatnani and Greenleaf, 2006).

\subsubsection{Co-transcriptional mRNA processing}

Several pre-mRNA processing events including 5' end capping, splicing and 3' end formation by cleavage and polyadenylation occur co-transcriptionally (Bentley, 2005; Sisodia et al., 1987). The CTD of RNAPII provides a landing pad for mRNA processing factors and plays a central role in the coupling of processing and 
transcription since the truncation of the CTD was shown to result in defects in capping, cleavage/polyadenylation and splicing (McCracken et al., 1997).

The first event of co-transcriptional processing during the transcription cycle takes place at the step of transcriptional initiation and requires Ser5-phosphorylated CTD. As already mentioned in section 1.1.3 on page 4ff. this modification is recognized by the capping machinery implicating the three different enzymes RNA triphosphatase, guanylyltransferase and 7-methyltransferase in yeast or rather two enzymes, the capping enzyme, that implicates triphosphatase and guanylyltransferase activities, and the 7methyltransferase in mammalian (Ho and Shuman, 1999; Schroeder et al., 2000; Shuman, 2001). It was shown that the human capping enzyme can stimulate promoter escape of RNAPII by relieving transcriptional repression by NELF and that its recruitment is enhanced by directly binding to SUPT5H (Mandal et al., 2004). Since human capping enzymes were found at 5' ends as well as throughout genes and even in 3' regions downstream of the poly(A) site, they seem to stay bound to the RNAPII platform after capping has taken place and may therefore even influence elongation, termination and 3' end processing (Glover-Cutter et al., 2008; Perales and Bentley, 2009).

Splicing factors are recruited to the nascent transcript as well and either remove most of the introns co-transcriptionally or mark others for post-transcriptional splicing (Wetterberg et al., 2001). For splicing U1 snRNP first binds to the GU sequence at the 5 ' splice site of the intron and U2 small nuclear RNA auxiliary factor (U2AF) to the AG 3' splice site. U2 snRNP then base pairs with the branch site, the triple snRNP U4$\mathrm{U} 6 / \mathrm{U} 5$ is recruited and rearranged to assume the catalytically active conformation as U1 and U4 are discarded (Perales and Bentley, 2009). U1 snRNP and other splicing regulatory proteins were shown to be recruited to genes and to interact with the CTD of RNAPII resulting in efficient splicing (Das et al., 2007; Listerman et al., 2006). Additionally, the knockdown of the chromodomain protein 1 (CHD1) and the decrease in H3K4me3 levels leads to a diminished association of U2 snRNP with chromatin and results in less efficient pre-mRNA splicing in vivo showing that $\mathrm{H} 3 \mathrm{~K} 4 \mathrm{me} 3$ is crucial for efficient recruitment of spliceosomal proteins via the CHD1 protein (Sims III et al., 2007).

Cleavage and polyadenylation of the transcript also occurs co-transcriptionally and is performed by a large cleavage/poly(A) complex. In yeast this complex is recruited to elongating RNAPII in part by binding of the components polyadenylation 
and cleavage factor subunit 11 (Pcf11p) which is a subunit of the cleavage factor 1A (CF1A) and the termination factor regulator of Ty1 transposition 103 (Rtt103p) to the Ser2-phosphorylated CTD (Kim et al., 2004; Licatalosi et al., 2002; Meinhart and Cramer, 2004; Proudfoot, 2004). In mammals the cleavage factor CPSF73 was found at both the 5' and 3' ends of genes and is thought to first bind with TFIID to the promoter and is then handed off to RNAPII and travels with it (Glover-Cutter et al., 2008).

Recently, purification of the human 3' end processing complex identified approximately 85 proteins including the polymerase associated factor (PAF) complex subunit cell division cycle 73 (CDC73) and the FACT subunit suppressor of Ty16 homolog (SUPT16H) implicating a connection between 3' end processing and transcriptional elongation in human cells (Shi et al., 2009). The cleavage and 3' end formation events in mammals will be explained in detail in the next sections.

\subsubsection{Mammalian 3' end processing of polyadenylated mRNAs}

In higher eukaryotes the canonical polyadenylation signal AAUAAA is recognized and bound by the cleavage and polyadenylation specificity factor (CPSF) that consists of at least five subunits (CPSF160, CPSF100, CPSF73, CPSF30 and FIP1). Another canonical sequence, the downstream sequence element (DSE) that is rich in $\mathrm{G} / \mathrm{U}$ or $\mathrm{U}$ residues is located up to $30 \mathrm{nt}$ downstream of the cleavage site and is bound by the cleavage stimulating factor (CSTF). After assembly of the basal 3' end processing machinery that consists of the cleavage factors I and II (CFI and CFII), CPSF73 catalyses the endonucleolytic cleavage at the cleavage site, 10-30 nt downstream of the poly(A) signal (Danckwardt et al., 2008; Shi et al., 2009; Weiner, 2005). Afterwards, the poly(A) polymerase (PAP) adds approximately 250 Anucleotides to the 3' end of the cleaved transcript (Danckwardt et al., 2008). Additional upstream or downstream signals can facilitate the cleavage that often occurs 3 ' to the dinucleotide CA (Weiner, 2005). CPSF was shown to directly interact with the transcription factor TFIID already being associated with transcription in the preinitiation complex (Dantonel et al., 1997).

In contrast to all other known eukaryotic mRNAs, replication-dependent histone mRNAs are not polyadenylated and their processing requires a different set of proteins. The details of histone mRNA 3' end processing and the functions of important factors being involved will be explained in section 1.3.2 on page $23 \mathrm{ff}$. 


\subsubsection{The importance of 3' end processing}

Eukaryotic pre-mRNA 3' end processing is a very important process that has several functions crucial for cell growth and viability. Polyadenylation of the 3' end of the pre-mRNA is important for its transport from the nucleus into the cytoplasm (Vinciguerra and Stutz, 2004). Furthermore, it functions in promoting stability of the mRNA by preventing degradation by 3' exonucleases which is a principle mechanism controlling mRNA stability (Wickens et al., 1997). Both, the addition of a poly(A) tail and the binding of the poly(A)-binding protein (PABP) has been shown to prevent degradation of mRNAs in the cytoplasm (Ford et al., 1997). The translation of mRNAs into proteins is enhanced by the interaction of the poly(A) tail and the PABP with the 5' cap (Wilusz et al., 2001). This interaction was shown to optimize the efficiency of translation in yeast (Preiss and Hentze, 1998). The 3' end processing machinery interacts with transcription factors of the preinitiation complex and with the CTD of RNAPII thus potentially playing a role in controlling transcriptional initiation. In addition, the poly(A) signal is crucial for proper transcriptional termination (Bentley, 2002; Proudfoot, 2004). Conversely, defects in 3' end processing can negatively influence transcription (Manley, 2002).

\subsection{Chromatin structure and histone modifications}

The genomic DNA of all eukaryotic cells is highly condensed and wrapped around core histone proteins to produce a condensed structure called the chromatin (Kornberg, 1974). One nucleosome, which is the fundamental unit of the chromatin structure, comprises a core of eight histone proteins: two each of histone $2 \mathrm{~A}(\mathrm{H} 2 \mathrm{~A})$ and 2B (H2B), histone $3(\mathrm{H} 3)$ and histone $4(\mathrm{H} 4)$ whereby two H2A-H2B dimers interact with an H3-H4 tetramer. Around these nucleosomes 147 base pairs of DNA are twined (Kornberg, 1977; Luger et al., 1997). The H1 histone serves as a linker that associates with DNA between single nucleosomes which leads to a high folded $30 \mathrm{~nm}$ fiber (Allan et al., 1980; Thoma et al., 1979). Histones are small basic proteins that consist of a flexible positively charged N-terminus the so-called "histone tail" and a globular $\mathrm{COOH}$-terminal domain that make up the nucleosome scaffold (Fischle et al., 2003; Jenuwein and Allis, 2001). The N-termini protrude away from the DNA and are thus exposed on the nucleosome surface (Luger et al., 1997). The majority of chromatin is found in a condensed, compact form called heterochromatin that contains only a few 
genes and is generally transcriptionally inactive. In comparison, the uncondensed regions of the genome that contain a high density of genes is referred to as euchromatin and generally more transcriptionally active (Henikoff, 2000; Richards and Elgin, 2002). Today it is known that not only the structure of chromatin but also posttranslational modifications of the histone tails are linked to transcriptional regulation.

\subsubsection{Histone modifications and the "histone code"}

Given their position of the surface of the nucleosomes the histone tails function as acceptors for a variety of enzyme-catalyzed posttranslational modifications taking place at specific amino acid side chains including acetylation, methylation, ubiquitination and sumoylation of lysine residues, phosphorylation of serine and threonine residues, and methylation of arginine residues (Fischle et al., 2003; Turner, 2007) (illustrated in Fig 2 on page 11). There are two possible mechanisms how posttranslational modifications of histones can regulate transcriptional activity. The first model predicts that the different chromatin modifications result in changes in electrostatic charge i.e. acetylation that neutralizes positive charge or phosphorylation that adds a negative charge or from modified interactions between nucleosomes (Strahl and Allis, 2000). This change would then affect the ability of nucleosomes to bind to DNA and thereby alter the accessibility for DNA binding proteins such as transcription factors. The second and probably most important model is the change of the nucleosome surface by the attached chemical poststranslational modifications that serves as a platform for specific chromatin-binding proteins (Berger, 2007). Two classes of domains that interact with specific modified residues are the bromodomain which binds to acetylated lysine residues and the chromodomain that interacts with methylated lysine residues (Berger, 2002). The hypothesis that a network of posttranslational modifications including the number, type, combination and localization within the genome controls the status of a gene thereby controlling the biological outcome is termed the "histone code" (Berger, 2002; Jenuwein and Allis, 2001; Strahl and Allis, 2000). In addition, histone modifications can influence each other in a synergistic or antagonistic way leading to a complex and diverse imprint pattern (Jenuwein and Allis, 2001). There are histone modifications that are linked to transcriptional activation and others for transcriptional repression. The most studied example, histone actetylation, is balanced by histone acetyltransferases (HATs) and histone deacetylases (HDACs) and is generally associated with the promoters of actively transcribed genes (Li et al., 2007). 
In the next section some specific other posttranslational modifications are pointed out and described in detail.

H2A

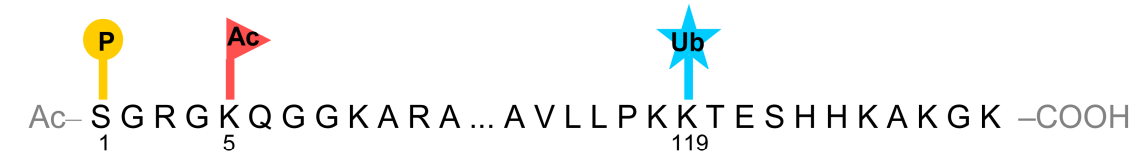

H2B

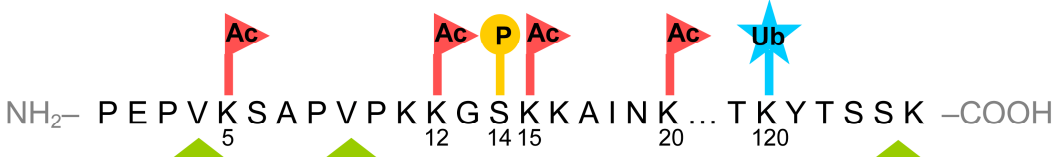

H3

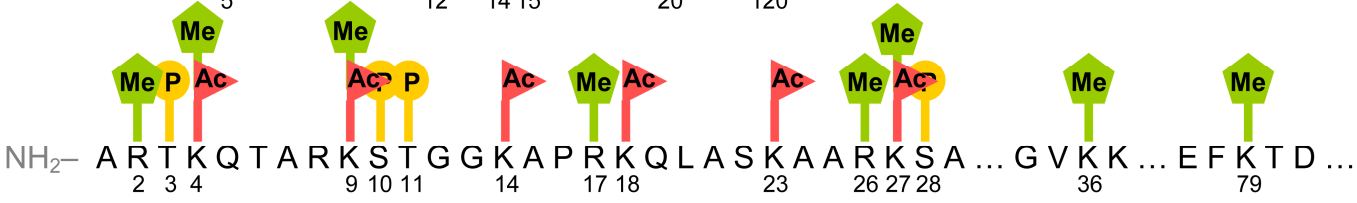

H4

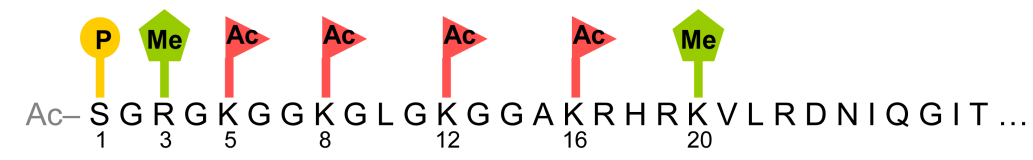

Fig 2: Posttranslational modifications of the core histones and their positioning. The histone tails can be methylated at lysines and arginines (green pentagons), phosphorylated at serines or threonines (yellow circles), acetylated at lysines (red triangles), or ubiquitinated at lysines (blue stars) (modified from Peterson and Laniel, 2004).

\subsubsection{Histone H2B monoubiquitination (H2Bub1)}

Monoubiquitination of histone $\mathrm{H} 2 \mathrm{~B}$ (H2Bub1) was shown to be associated with transcriptionally active DNA and appears to occur primarily co-transcriptionally (Davie and Murphy, 1990; Osley, 2006). In humans H2Bub1 is preferentially found in the transcribed regions of highly expressed genes and was reported in yeast to be required for efficient reassembly of nucleosomes during elongation. Therefore H2Bub1 was suggested to be linked to transcriptional elongation in both yeast and humans (Fleming et al., 2008; Minsky et al., 2008).

The ubiquitination process requires three separate enzymatic activities (Hochstrasser, 1996). First, ubiquitin, a 76 amino acid protein, is activated by an ubiquitin activating enzyme (E1) in an ATP-dependent manner. Secondly, it is conjugated to a cysteine residue of a ubiquitin conjugating enzyme (E2) via a thioester bond and finally transferred to a target lysine residue by a ubiquitin-protein-isopeptide ligase (E3) (Weake and Workman, 2008). Whereas polyubiquitination generally targets a protein for degradation via the $26 \mathrm{~S}$ proteasome, monoubiquitination is generally associated with a change in protein function (Pickart, 2001). 
In yeast (Saccharomyces cerevisiae) H2Bub1 takes place at lysine residue 123 (K123) whereas in humans it occurs at lysine residue 120 (K120) (Osley, 2006). Less than $10 \%$ of the total histone $\mathrm{H} 2 \mathrm{~B}$ are monoubiquitinated and this modification is rapidly removed by ubiquitin proteases (UBPs) and is thus a highly dynamic process (Osley, 2004; Zhang, 2003). In yeast, Ubp8p, a component of the Spt-Ada-Gen5acetyltransferase (SAGA) complex deubiquitinates $\mathrm{H} 2 \mathrm{~B}$ in vitro and in vivo and in humans ubiquitin specific protease 22 (USP22), a subunit of the human SAGA complex was shown to be the ortholog of yeast Ubp8p (Henry et al., 2003; Zhang et al., 2008). Interestingly, both ubiquitination and deubiquitination of histone $\mathrm{H} 2 \mathrm{~B}$ appear to be involved in transcriptional activation (Henry et al., 2003).

In yeast, the E2 enzyme for H2Bub1 in vivo was shown to be radiation-sensitive mutant protein $6(\operatorname{Rad} 6 \mathrm{p})$ and the interacting E3 ligase was identified to be a RING finger protein called brefeldin A sensitive protein 1 (Bre1p) (Hwang et al., 2003; Robzyk et al., 2000; Wood et al., 2003a). Based on sequence alignment two homologs for the yeast Rad6p protein have been found in humans, namely hHR6A and hHR6B or also called UBE2A and UBE2B according to the new nomenclature (Koken et al., 1991). Up to now it is debated if these are the E2 enzymes responsible for H2Bub1 in vivo or if rather another ubiqutin conjugase, UBCH6, could be implicated in this process (Pavri et al., 2006; Zhu et al., 2005b). Concerning the E3 ligase two RING finger proteins, RNF20 and RNF40, that share sequence homology with yeast Bre1p were shown to both be essential for monoubiquitination of histone H2B (Kim et al., 2009; Zhu et al., 2005b).

Rad6p can associate with the elongating form of RNAPII and this interaction is dependent upon Bre1p and the Paf1p complex in yeast (Xiao et al., 2005). The yeast Paf1p complex, consisting of five different proteins (Paf1p, Rtf1p, Ctr9p, Cdc73p and Leo1p) was identified to bind to Ser5-P CTD of RNAPII and to interact with Bre1p to recruit Rad6p to the promoter region of actively transcribed genes (Laribee et al., 2007). Rad6p is also phosphorylated and thereby activated by the Bur1p/Bur2p kinase complex which in this way is implicated in controlling monoubiquitination of H2B (Wood et al., 2005; Wood and Shilatifard, 2006). These observations show that H2Bub1 is a histone mark that is coupled to RNAPII transcriptional elongation in both yeast and humans. 


\subsubsection{Histone $\mathrm{H3}$ lysine 4 trimethylation (H3K4me3)}

Methylation of histones occurs either on arginine and/or lysine residues, whereas arginine methylation of histones is involved in gene activation but lysine methylation instead can influence either activation or repression (Berger, 2002). The lysine residues of histone $\mathrm{H} 3$ or $\mathrm{H} 4$ are methylated by lysine methylating transferases (KMTs) and can be mono- di- or trimethylated. Each level of modification can have a different biological outcome showing the complexity of this posttranslational modification (Berger, 2007).

Trimethylation of histone $\mathrm{H} 3$ (H3K4me3) is associated with highly transcriptionally active genes whereas dimethylation of histone $\mathrm{H} 3$ (H3K4me2) occurs at both inactive and active genes (Berger, 2007; Bernstein et al., 2002; Santos-Rosa et $a l$., 2002). Concerning the localization of this posttranslational modification, it was shown that $\mathrm{H} 3 \mathrm{~K} 4 \mathrm{me} 3$ occurs near the transcription start site (TSS) and the 5' end of the open reading frame $(\mathrm{ORF}), \mathrm{H} 3 \mathrm{~K} 4 \mathrm{me} 2$ is found throughout the whole body of the gene but peaks in the middle and H3K4me1 is enriched at the 3' end of genes (Li et al., 2007; Pokholok et al., 2005). Mono-, di- and trimethylation of histone $\mathrm{H} 3$ is performed by the complex of proteins associated with Set1p (COMPASS) in yeast, a homolog of the human methyltransferases SET1 and the mixed lineage leukemia (MLL) protein family (Schneider et al., 2005). Human MLL complexes contain the subunits absent, small, or homeotic-like protein (ASH2L), retinoblastoma binding protein 5 (RBBP5) and WD repeat domain 5 protein (WDR5) and knockdown of ASH2L results in a global reduction of H3K4me3 (Steward et al., 2006). The MLL1 gene is involved in numerous translocations that are found in several human acute leukemias (Rowley, 1998; Tenney and Shilatifard, 2005). The fusion proteins of such translocated $M L L$ genes loose their H3K4 methyltransferase domain but are still able to bind to homeobox $(H O X)$ genes and are associated with an increase in their expression and thus dysregulation (Guenther et al., 2005; Krivtsov et al., 2008; Rozovskaia et al., 2001).

Since H3K4 methylation does not affect transcription elongation in vitro per se and in addition the methyltransferase Set1p does not affect the processivity of RNAPII in yeast in vivo, the precise function of $\mathrm{H} 3 \mathrm{~K} 4$ methylation in transcription regulation is not yet known (Mason and Struhl, 2003; Pavri et al., 2006). The importance of H3K4 methylation thus seems to lie primarily in a signalling function (Li et al., 2007). This is supported by the fact that chromatin-remodeling factors and histone modification complexes that contain plant homeodomain (PHD) fingers can directly recognize and 
bind to H3K4me3 (Zhang, 2006) and recruit complexes to activate or repress transcription.

The methylation status of $\mathrm{H} 3 \mathrm{~K} 4$ plays a role in either recruiting, activating or repressing effectors. $\mathrm{H} 3 \mathrm{~K} 4 \mathrm{me} 2$ and $\mathrm{H} 3 \mathrm{~K} 4 \mathrm{me} 3$ both recruit acetyltransferases directly or via binding of Chd1p, an elongation-related chromatin-remodeling factor, and the methyltransferase Set1p as part of COMPASS which leads to an increase in other histone acetylations and trimethylated H3K4 respectively (Berger, 2007; Pray-Grant et al., 2005; Sims III and Reinberg, 2006). In humans CHD1 associated with H3K4me3 assists in recruiting pre-spliceosomal components like U2 snRNP to chromatin resulting in facilitating pre-mRNA maturation (Sims III et al., 2007; Sims III and Reinberg, 2009). Importantly, via this posttranslational modification the rate but not the catalysis of splicing in human cells is affected. Thus, the role of $\mathrm{H} 3 \mathrm{~K} 4 \mathrm{me} 3$ seems to lie mainly in serving as a mark for proteins that are implemented in the recruitment of complexes that influence transcription or pre-mRNA maturation.

In contrast, only $\mathrm{H} 3 \mathrm{~K} 4 \mathrm{me} 3$ serves as a signal to recruit repressing effectors like deacetylases and demethylases leading to repression of gene activity (Berger, 2007; Shi et al., 2006; Huang et al., 2006). Like this, the posttranslational modification of one residue on a histone can have multiple biologic outcomes.

\subsubsection{Histone H3 lysine 36 trimethylation (H3K36me3)}

Di- and trimethylation of lysine 36 in histone H3 (H3K36me2 and H3K36me3) are both coupled to transcriptional elongation.

In Saccharomyces cerevisiae the histone methyltransferase Set2p was found to be the specific enzyme for methylating histone $\mathrm{H} 3$ on lysine residue 36 (di- and trimethylation) (Strahl et al., 2002). It is recruited to the CTD phosphorylated by the Ctk complex (Wood and Shilatifard, 2006). Set2p was shown to directly interact with elongating RNAPII by binding to dual P-Ser2/P-Ser5 phosphorylated CTD but not to unphosphorylated RNAPII in vitro (Li et al., 2003; Xiao et al., 2003). In addition, ChIP analysis revealed the association of Set2p and H3K36 methylation with the coding region of genes and the correlation with active transcription (Krogan et al., 2003).

In Drosophila melanogaster H3K36me2 peaks near the promoter region of genes whereas H3K36me3 is accumulated towards the 3' end of genes. Interestingly, these methylation events are performed by two differents KMTs, namely maternal effect sterile family member 4 (dMes-4) and huntingtin interacting protein b (dHypb) 
respectively (Bell et al., 2007). In humans the methyltransferases HYPB, SET and MYND domain containing 2 (SMYD2) and nuclear receptor-binding SET domaincontaining protein 1 (NSD1) appear to take over these roles since they can methylate H3K36 in vitro (Brown et al., 2006; Edmunds et al., 2008; Rayasam et al., 2003; Sun et al., 2005). The enzyme that is relevant in vivo and if it catalyses mono-, di- or trimethylation of H3K36 still remains to be elucidated.

Concordantly, these studies show that in both yeast and mammals, P-Ser2 of the CTD is necessary to recruit the histone methyltransferase responsible for the trimethylation of H3K36 (Krogan et al., 2003; Li et al., 2005a).

The role of H3K36me3 during transcriptional elongation was shown in yeast to recruit histone deacetylases specifically to the transcribed region in order to suppress improper internal initiation (Carrozza et al., 2005). Otherwise transcription initiation on cryptic promoters in the body of genes would lead to 5' truncated transcripts and thus aberrant protein production.

\subsubsection{Histone modification crosstalk}

Histone modifications can influence one another. For example, one modification can function to recruit an enzyme or activate an enzymatic activity that is necessary to generate another histone modification (Suganuma and Workman, 2008). This so-called trans-histone effect is well known for $\mathrm{H} 2 \mathrm{Bub} 1$ in being a prerequisite for $\mathrm{H} 3 \mathrm{~K} 4 \mathrm{me} 3$ and H3K79me3 and is highly conserved from yeast to human (Dover et al., 2002; Fischle et al., 2003; Ng et al., 2003; Sun and Allis, 2002; Wood et al., 2005). Rad6p was shown to be essential for $\mathrm{H} 3 \mathrm{~K} 4 \mathrm{me} 3$ through $\mathrm{H} 2 \mathrm{Bub} 1$ on lysine 123 in yeast in a unidirectional regulatory pathway (Sun and Allis, 2002). Not only H3K4me3 by the Set1p-containing COMPASS complex but also H3K79me3 by a methyltransferase called disruptor of telomeric silencing 1 (Dot1p) was shown to be dependent upon H2Bub1 (Briggs et al., 2002; Ng et al., 2002; Singer et al., 1998). Interestingly, H2Bub1 specifically affects diand trimethylation of $\mathrm{H} 3 \mathrm{~K} 4$ and $\mathrm{H} 3 \mathrm{~K} 79$ but not monomethylation in yeast. Thus, it is thought that not the enzyme recruitment is affected but in fact the processivity of the histone metyltransferase (Dehe et al., 2005; Shahbazian et al., 2005). By using chemically ubiquitinated $\mathrm{H} 2 \mathrm{~B}$, McGinty et al. recently showed that H2Bub1 directly activates DOT1L-mediated intranucleosomal methylation of H3K79 in humans and that this posttranslational modification is carried out by the catalytic domain of DOT1L (McGinty et al., 2008). However, the recruitment of DOT1L was not dependent upon 
monoubiquitination of $\mathrm{H} 2 \mathrm{~B}$ in their studies. In contrast to $\mathrm{H} 3 \mathrm{~K} 4 \mathrm{me} 3$ and $\mathrm{H} 3 \mathrm{~K} 79 \mathrm{me} 3$, H3K36 methylation was shown not to be dependent on the H2Bub1 driven transhistone pathway (Briggs et al., 2002).

\subsubsection{Movement through chromatin is coupled to posttranslational histone modifications}

For efficient transcriptional elongation through chromatin the physical barrier caused by the nucleosomes has to be removed. This process is done by nucleosome assembly/disassembly factors called histone chaperones which are defined as factors that have a histone binding activity and a histone-dependent, ATP-independent nucleosome assembly activity (Eitoku et al., 2008). Most histone chaperones have a preference for either binding $\mathrm{H} 2 \mathrm{~A} / \mathrm{H} 2 \mathrm{~B}$ or $\mathrm{H} 3 / \mathrm{H} 4$ dimers. These escort proteins transfer histones from one chaperone to another, to enzymes, onto or off DNA and are thus crucial in fundamental processes like transcription, replication and DNA repair (De Koning et al., 2007).

The FACT complex is a heterodimeric complex consisting of the structure specific recognition protein 1 (SSRP1) and the SUPT16H protein in humans and polymerase I binding protein 3 (Pob3p) and Spt16p in yeast and is a histone chaperone implicated in transient transcription-coupled nucleosome disassembly (Belotserkovskaya and Reinberg, 2004; Orphanides et al., 1998; Orphanides et al., 1999). During transcriptional elongation it removes a single $\mathrm{H} 2 \mathrm{~A} / \mathrm{H} 2 \mathrm{~B}$ dimer from the nucleosomes thus decreasing the barrier to elongating RNAPII (Reinberg and Sims III, 2006). This process was shown to be stimulated by monoubiquitination of H2B (Pavri et al., 2006).

Additionally, by travelling with elongating RNAPII, FACT is also involved in the reassembly of nucleosomes during transcriptional elongation (Mason and Struhl, 2003). Interestingly, the FACT and the PAF complex have been shown to genetically and physically interact in yeast (Krogan et al., 2002). The following model for the role of H2Bub1 in transcription elongation was created by Pavri and co-workers based on in vitro transcription elongation assays. When RNAPII halts at the first nucleosome, FACT is recruited and further recruits the PAF complex and the ubiquitination machinery (UBCH6 and RNF20/40) leading to H2Bub1. This histone modification then facilitates the function of FACT resulting in an increased $\mathrm{H} 2 \mathrm{~A} / \mathrm{H} 2 \mathrm{~B}$ dimer removal thus leading to a more accessible DNA template and hence more efficient transcriptional 
elongation by RNAPII (Pavri et al., 2006). In contrast, in vivo it was shown that the FACT subunit Spt16p and $\mathrm{H} 2 \mathrm{Bub1}$ regulate histone deposition at the yeast galactokinase 1 gene (gall) during elongation and prevent internal initiation from cryptic promoters in gene-coding regions (Fleming et al., 2008). Thus, although it is debated whether FACT is involved in adding or removing $\mathrm{H} 2 \mathrm{~A} / \mathrm{H} 2 \mathrm{~B}$ dimers, it clearly plays an important role in altering the cohesion of the chromatin.

\subsubsection{Poised promoters show hallmarks of transcription initiation}

Studies on mammalian promoters revealed that preassembled PICs can be found at many inactive genes and that these genes display histone marks that are generally associated with transcription initiation (Kim et al., 2005b; Lee et al., 2006). Additionally, comparison of transcript levels with the location of RNAPII and histone marks in differentiated mammalian cells, embryonic stem cells and Drosophila cells showed that hallmarks of initiation can be found in many promoters regardless of transcriptional output (Guenther et al., 2007; Muse et al., 2007; Zeitlinger et al., 2007). These marks include RNAPII occupancy, H3K4me3 and acetylation of H3K9 and $\mathrm{H} 3 \mathrm{~K} 14$ and are found on approximately $75 \%$ of all protein-coding genes (Agalioti et al., 2002; Margaritis and Holstege, 2008). However, only a subset of these genes produce full-length transcripts and possess hallmarks of elongation like H3K36me3 and H3K79me2 (Guenther et al., 2007). This suggests that not only active or inactive genes exist but an additional third class, so-called "poised" genes, which produce no detectable transcripts but experience transcription initiation (Price, 2008). The phosphorylation profile of the CTD and the histone modification profile in active and "poised" genes could be revealed by ChIP analysis using specific antibodies. They showed that active genes possess both P-Ser2 and P-Ser5 CTD whereas only P-Ser5 was detected in "poised" genes. The histone modification profile showed H3K4me3 and $\mathrm{H} 3 \mathrm{~K} 36 \mathrm{me}$ in active genes and H3K4me3 and H3K27me3, implemented by the polycomb repressive complex 2 (PRC2), in “poised” genes (Brookes and Pombo, 2009).

Many developmentally regulated promoters in mammalian embryonic stem cells were shown to possess bivalently marked chromatin i.e. both the activating mark H3K4me3 and the repressing mark H3K27me3 (Bernstein et al., 2006; Mikkelsen et al., 2007). In this "bivalent domain" model the repressive mark is dominant over the activating so that the genes are held in a "poised" state for activation during development leading to very low transcription levels (Herz et al., 2009). However, the 
presence of the activating marks allow for rapid activation of gene expression following removal of the repressive marks. In differentiating neurons from these stem cells $\mathrm{H} 3 \mathrm{~K} 4 \mathrm{me} 3$ persists but $\mathrm{H} 3 \mathrm{~K} 27 \mathrm{me} 3$ gets lost in genes that get avtivated whereas in genes that remain inactive, $\mathrm{H} 3 \mathrm{~K} 27 \mathrm{me} 3$ stays constant and $\mathrm{H} 3 \mathrm{~K} 4 \mathrm{me} 3$ is removed (Berger, 2007).

In summary, the observations depicted here link the transcriptional status of a gene to a specific phosphorylation pattern of the RNAPII CTD and a distinct histone modification pattern that connects transcriptional regulation with posttranslational histone modifications.

\subsection{Coupling of histone synthesis with the cell cycle}

The mammalian cell cycle consists of two major stages, mitosis ( $\mathrm{M}$ phase) in which nuclear division takes place including pro-, meta-, ana-, and telophase and the interphase between two $M$ phases which is divided into $G_{1}, S$ and $G_{2}$ phases (Norbury and Nurse, 1992). $G_{1}$ and $G_{2}$ are the gap phases before and after $S$ phase in which DNA replication occurs (Mitchison, 1971). After cytokinesis, cells may enter into a resting state that is known as "quiescence" or $\mathrm{G}_{0}$ phase and thus stop proliferating (Malumbres and Barbacid, 2005; Vermeulen et al., 2003). The cell cycle requires three major transitions at the beginning of $S$ phase $\left(G_{1} / S\right)$, entry of mitosis $\left(G_{2} / M\right)$ and at the exit of mitosis (metaphase/anaphase). The $\mathrm{G}_{1} / \mathrm{S}$ transition is marked by the onset of DNA replication, $\mathrm{G}_{2} / \mathrm{M}$ transition is accompanied by breakdown of the nuclear envelope and chromatin condensation and the metaphase/anaphase transition is marked by the separation of the sister chromatids (Hochegger et al., 2008).

\subsubsection{Proteins that regulate the progression of the cell cycle}

Each transition from one cell cycle phase to another is highly regulated by the interplay of different molecules. Among these the cyclins are the key regulators that together with cyclin-dependent kinases (CDKs) form activated Serine/Threoninekinases that phosphorylate target proteins leading to progression through the cell cycle. Importantly, cyclins are degraded in a specific timely regulated manner with half-lives of hours or rather seconds (Kobayashi et al., 1994). In addition, kinases, phosphatases, CDK inhibitors and specific ubiquitin-proteasome complexes are necessary to directly regulate the activity of the Cyclin/CDK complexes. 


\subsubsection{Cyclins and cyclin-dependent kinases (CDKs)}

Two of the most important proteins that are involved in the cell cycle regulation are cyclins and cyclin-dependent kinases (CDKs). Complexes of cyclins and CDKs are formed during specific cell cycle phases (illustrated in Fig 3 on page 19) and phosphorylate important cell cycle regulating target proteins. The mammalian cyclins are the $\mathrm{G}_{1}$ cyclins Cyclin D (D1, D2 and D3) and Cyclin E (E1 and E2), and the mitotic cyclins Cyclin A (A1 and A2) and Cyclin B (B1 and B2) (Giacinti and Giordano, 2006). The first cyclins produced in the cell cycle in response to extracellular signals, the Dtype cyclins, preferentially bind to CDK4 or CDK6 and are then crucial for the $G_{1} / S$ transition by regulating early $\mathrm{G}_{1}$ phase whereas the Cyclin E/CDK2 complex is necessary for trigerring $S$ phase i.e. initiation of DNA replication (Hitomi and Stacey, 1999). Cyclin A/CDK2 and Cyclin A/CDK1 are needed in the final steps of $S$ phase and in $G_{2}$ phase and $C y c l i n B / C D K 1$ regulates several events during the $G_{2} / M$ transition and progression through mitosis (Giacinti and Giordano, 2006; Hochegger et al., 2008; Malumbres and Barbacid, 2005; Nigg, 2001; Nurse, 2000).

A

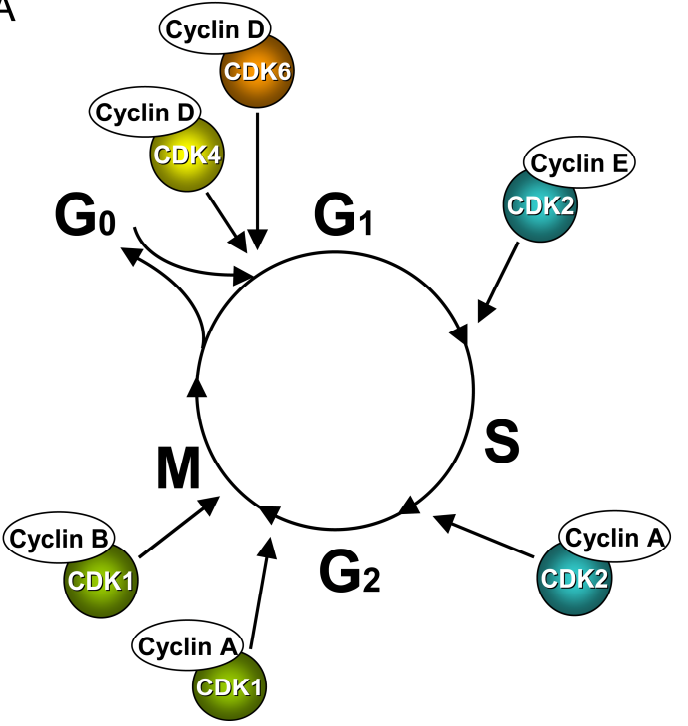

B

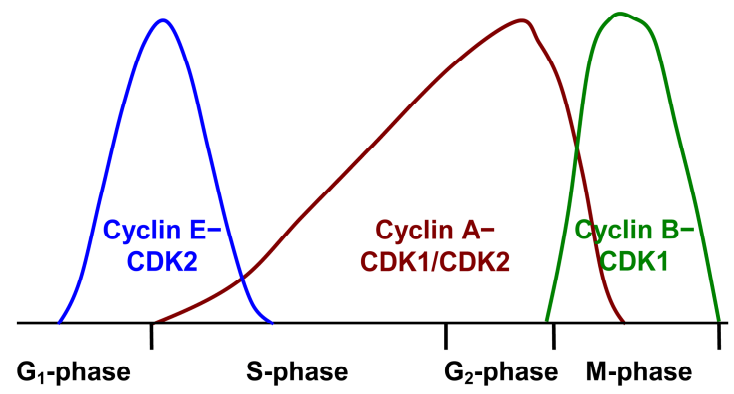

Fig 3: The mammalian cell cycle. A circular (A) and a linear (B) model illustrate the different Cyclin/CDK complexes and their function throughout the mammalian cell cycle. D-type cyclins and CDK4 or CDK6 regulate events in early $G_{1}$ phase, Cyclin $E / C D K 2$ is important for $G_{1} / S$ transition, Cyclin A/CDK2 and Cyclin A/CDK1 play a role during $S$ and $\mathrm{G}_{2}$ phase and Cyclin B/CDK1 is responsible for mitosis (modified from Hochegger et al., 2008; Vermeulen et al., 2003).

In order that they are only active during the cell cycle phase they are needed, the cyclins are synthesized and degraded during each cell cycle and their levels are finely modulated by the ubiquitin-proteasome system (UPS) (Besson et al., 2008). More 
precisely, they are ubiquitinated by E3 ubiquitin ligases, the SKP1-CUL1-F-box-protein complex (SCF) and the anaphase-promoting complex/cyclosome (APC/C) leading to their degradation by the UPS (Fasanaro et al., 2010). The SCF complex is involved in all cell cycle stages and targets many key regulators of the cell cycle like Cyclin D1, Cyclin E1, the pocket protein family member p130 and the transcription factor E2F1 as well as cyclin-dependent kinase inhibitors (CKIs) but plays a prominent role in $\mathrm{G}_{1} / \mathrm{S}$ transition. In contrast, the APC/C complex orchestrates progression through mitosis into $\mathrm{G}_{1}$ phase by inducing the degradation of Cyclin B and the segregation of chromosomes during anaphase (Fasanaro et al., 2010; Hochegger et al., 2008; Wickliffe et al., 2009).

Another level of regulation of Cyclin/CDK complexes is their inactivation through binding to CKIs. In metazoans two CKI families exist which include the inhibitors of CDK4 (INK4) family and the Cip/Kip family (Besson et al., 2008). The INK4 family consists of $\mathrm{p} 16^{\mathrm{INK} 4 \mathrm{a}}, \mathrm{p} 15^{\mathrm{INK} 4 \mathrm{~b}}, \mathrm{p} 18^{\mathrm{INK} 4 \mathrm{c}}$ and $\mathrm{p} 19^{\mathrm{INK} 4 \mathrm{~d}}$ that all bind to CDK4 and CDK6 thus preventing their association with D-type cyclins. Members of the Cip/Kip family, p2 $1^{\mathrm{Cip} 1 / \mathrm{Waf} 1 / \mathrm{Sdi} 1}, \mathrm{p} 27^{\mathrm{Kip} 1}$ and $\mathrm{p} 57^{\mathrm{Kip} 2}$, instead bind to both cyclins and CDK subunits and regulate the activity of Cyclin D-, E-, A- and B/CDK complexes (Sherr and Roberts, 1999).

Moreover, the activity of Cyclin/CDK complexes is further regulated by kinases and phosphatases. For their complete activation they have to be phosphorylated at a conserved threonine residue by a CDK activating kinase (CAK). In contrast, the "small" mutant protein 1 (WEE1) exists that adds an inhibitory phosphate to a specific tyrosine residue (Malumbres and Barbacid, 2005). Finally, phosphatases can either deactivate or activate CDKs by removing either an activating or an inhibitor phosphate respectively (Morgan, 1995). The CDC25 phosphatase family consists of CDC25A, CDC25B and CDC25C. CDC25A plays an important role in the $\mathrm{G}_{1} / \mathrm{S}$ transition and CDC25B is activated during $\mathrm{S}$ phase and activates the mitotic Cyclin B/CDK1 by removing an inhibitory phosphate. Active Cyclin B/CDK1 then phosphorylates and activates CDC25C leading to a positive feedback mechanism and to entry into mitosis (Aressy and Ducommun, 2008; Nilsson and Hoffmann, 2000; Vermeulen et al., 2003).

In addition to being highly regulated by the depicted mechanisms, Cyclin/CDK complexes themselves regulate a large number of target proteins in the cell by phosphorylation of Ser/Thr residues of the respective proteins. In the next section the retinoblastoma protein $(\mathrm{RB})$ will be described as an important target protein and its pathway will be presented in more detail. 


\subsubsection{The retinoblastoma protein $(\mathrm{pRB})$}

During $\mathrm{G}_{1}$ phase Cyclin D binds to CDK4 or CDK6 and forms a complex that phosphorylates the hypophosphorylated retinoblastoma protein (RB) leading to its hyperphosphorylation and thus inactivation (Weinberg, 1995).

The retinoblastoma gene was the first identified tumor suppressor gene and was isolated from retinoblastoma tumors (Lee et al., 1987). The encoded retinoblastoma protein is a nuclear phosphoprotein that belongs to the pocket protein family consisting of three proteins RB, p107 and p130 that all contain a highly conserved sequence in their pocket domain (Poznic, 2009). RB represses transcription of genes that are necessary for the $G_{1} / S$ transition and thus arrests cells in the $G_{1}$ phase of the cell cycle (Weinberg, 1995). RB can be phosphorylated by Cyclin D together with CDK4 or CDK6 or Cyclin E/CDK2 and there is evidence that both of these complexes are needed to completely inactivate RB (Harbour and Dean, 2000; Lundberg and Weinberg, 1998). Dependent upon its phosphorylation status, RB regulates the activity of the E2F family of transcription factors which are crucial for the expression of target genes that play a role in cell cycle progression.

\subsubsection{The E2F transcription factor family members}

The E2F family consists of at least eight family members, E2F1-E2F8 that have functional and structural differences (DeGregori and Johnson, 2006). Most of them can interact with members of the retinoblastoma pocket protein family. There are for example transcriptional activators (E2F1, E2F2, E2F3) that interact with $\mathrm{pRB}$ and transcriptional repressors (E2F4 and E2F5) that mostly bind to p130 and p107 (Dyson, 1998; Gaubatz et al., 2000). E2F6, E2F7 and E2F8 instead are transcriptional repressors that function through pRB-independent mechanisms (Polager and Ginsberg, 2009). In the cell free E2Fs and complexed E2Fs exist that are either in an activated or repressed state respectively. Free E2Fs are heteromeric consisting of a E2F family member subunit and a dimerization partner (DP) protein subunit (DP1 or DP2) that potentiates the activity of the E2F factor and stabilizes its interaction with the hypophosphorylated RB protein (Bandara et al., 1993; Helin et al., 1993; Krek et al., 1993; Ormondroyd et al., 1995). The complexed forms instead contain E2F/DP heterodimers that are stably bound to the respective RB family member (pRB, p130, p107) and thus kept in an 
inactive state (Cao et al., 1992; Chellappan et al., 1991; Cobrinik et al., 1993; Shirodkar et al., 1992).

As already mentioned, the activity of the E2F transcription factor depends on the phosphorylation status of its putative RB binding partner. In its active, hypophosphorylated form, $\mathrm{RB}$ is bound to E2F/DP and thus keeps it in an inactive complex. If $\mathrm{RB}$ is phosphorylated the $\mathrm{E} 2 \mathrm{~F} / \mathrm{DP} / \mathrm{RB}$ complex dissociates allowing E2F to activate target genes that encode for cell cycle regulators like Cyclin E, Cyclin A, CDK2 and factors necessary for entering S phase like DNA Polymerase $\alpha$, thymidine kinase, etc. (Geng et al., 1996; Huet et al., 1996; Ogris et al., 1993; Ohtani et al., 1995; Pearson et al., 1991; Schulze et al., 1995).

Considering many studies, three general types of E2F complexes seem to exist: activator E2F complexes (E2F/DP), inhibited E2F complexes (E2F/DP/RB) and repressor E2F complexes (E2F/DP/RB). In the activator complexes the activation domain of E2F promotes transcription, in the inhibited complexes the bound RB masks the activator domain leading to an inert complex and in the repressor complexes the bound RBs recruited to promoters have a repressor activity (Dyson, 1998).

During the cell cycle different E2Fs are expressed in different phases of the cell cycle. The E2F activators are highly expressed from $G_{1}$ to $S$ phase with E2F3 expression increasing in early $G_{1}$ to mid $G_{1}$ phase and E2F1 and E2F2 being most highly expressed at the $G_{1} / S$ boundary (Johnson and Schneider-Broussard, 1998). In contrast, the E2F repressors E2F4 and E2F5 are expressed throughout the cell cycle but in $G_{0}$ and early $G_{1}$ they are kept in repressive complexes with p107 and p130 and are recruited to the nucleus (De la Luna et al., 1996). There the repressor complexes are thought to bind to promoters and to recruit HDACs resulting in transcriptional repression of $S$ phase genes (Wilson, 2007). When cells enter late $G_{1}$ phase the repressor complexes are rapidly replaced by the activator complexes containing the subunit E2F1, E2F2 or E2F3 which then leads to the expression of genes important for the $\mathrm{G}_{1} / \mathrm{S}$ transition (Takahashi et al., 2000). Recently, it was shown that in late $\mathrm{G}_{1}$ phase the activator E2F1 recruits the cofactor host cell factor-1 (HCF-1) that associates with the H3K4 methyltransferase complex SET1/MLL leading to a facilitated activation of $S$ phase genes (Tyagi et al., 2007). 


\subsubsection{Histone synthesis is strictly coupled to the cell cycle}

During the cell cycle the correct packaging of DNA into chromatin has to be sustained and thus the histone proteins have to be rapidly produced during $\mathrm{S}$ phase of a replicating cell to package the newly synthesized DNA into nucleosomes before the cell divides (Osley, 1991). Replication-dependent histone mRNA levels increase up to 35fold in the beginning of $\mathrm{S}$ phase and drop to normal levels in the end of $\mathrm{S}$ phase (Harris et al., 1991). This S phase-dependent accumulation of histone transcripts is due to an increase in the rate of transcription and in the processing efficiency and the reduction to basal levels in the end of S phase is explained by a rapid degradation of the transcripts and inactivated processing (Marzluff et al., 2008). The factors involved in the coupling of histone mRNA transcription to $\mathrm{S}$ phase of the cell cycle will be introduced in the next sections of this chapter.

\subsubsection{Replication-dependent histone genes: organization and localization}

In the genome of all studied metazoans the five canonical histone genes are organized in clusters that contain multiple copies of each (Marzluff et al., 2008). In mammals there are three clusters, the largest one termed HISTI which is located on chromosome 6 (6p21-6p22) in humans and two smaller clusters HIST2 located on chromosome 1 (1q21) and HIST3 located at 1q42 in humans (Marzluff et al., 2002). These clusters are further separated into either tandemly repeated or jumbled clusters (Marzluff et al., 2008). The first one contains one copy of each of the five histone gene whereas there is no gene order and also no identical genes for each protein in the jumbled cluster. While tandemly repeated clusters are found in Drosophila melanogaster and frogs, jumbled clusters are found in mammals and birds (Marzluff et $a l ., 2008)$. Histone gene loci were observed to be frequently associated with Cajal bodies (CBs), subnuclear organelles first reported by Ramón y Cajal in 1903 that contain factors involved in histone mRNA 3' end processing (Cajal, 1903; Frey and Matera, 1995; Wu and Gall, 1993). However, recent publications have revealed that the principal regulatory proteins important for histone gene expression as well as 3' end processing and histone genes are concentrated at specific subnuclear compartments called histone locus bodies (HLBs) that were first described in Drosophila melanogaster and that in comparison the mentioned factors are less frequently present in coilin-containing CBs (Ghule et al., 2008; Liu et al., 2006; Matera, 2006). Moreover, 
HLBs are associated with replication-dependent histone gene clusters on chromosomes 1 and 6 and the degree of their colocalization with CBs depends on the cell line and the cell cycle phase (Bongiorno-Borbone et al., 2010).

The two histone genes, HIST1H2BD and HIST1H2AC, addressed most extensively in this thesis, are both located at the major histone gene locus on chromosome 6. There are 15 HIST1H2B and 12 HIST1H2A genes that are named alphabetically with the HISTIH2BA gene being closest to the telomere and the $15^{\text {th }}$ gene of this cluster lying closest to the centromer (Marzluff et al., 2002). The expression of the histone genes HISTIH2BD and HISTIH2AC was shown to be downregulated by knockdown of RNF20, the E3 ubiquitin ligase of histone H2B, in HeLa cells, with HIST1H2BD being the histone gene that was affected to the highest extent (Shema et $a l .$, 2008).

\subsubsection{Replication-dependent histone mRNA 3' end processing}

Unlike all other known mRNAs in eukaryotic cells, the mRNAs transcribed from canonical replication-dependent histone genes do not contain introns and are not polyadenylated (Dominski and Marzluff, 1999; Marzluff et al., 2008). Constitutively expressed histone mRNAs which encode the replacement variant histones are not cell cycle regulated but are instead polyadenylated and some of them even contain introns (Brush et al., 1985; Wells and Kedes, 1985).

Instead of a poly(A) tail the replication-dependent histone mRNAs end in a highly conserved 16 nucleotide long 3' end stem-loop structure, which is located 4-5 nucleotides upstream of the cleavage site. This cleavage site is followed by a purine-rich element the so-called histone downstream element (HDE) with the consensus sequence AAAGAG in vertebrates (Birnstiel et al., 1985; Dominski and Marzluff, 1999; Marzluff, 1992; Marzluff et al., 2008).

The formation of the histone mRNA 3'end requires the binding of the stem-loop binding protein (SLBP) to the conserved stem-loop structure and the interaction of the U7 snRNA, a component of the U7 small nuclear ribonucleoprotein (snRNP) with the HDE (Dominski and Marzluff, 1999; Mowry and Steitz, 1987) (illustrated in Fig 4 on page 26). The cleavage occurs 4-5 nucleotides after the stem-loop, 3' to the dinucleotide CA and is performed by CPSF73 which also cleaves polyadenyaletd mRNAs (Dominski et al., 2005; Gick et al., 1986; Weiner, 2005). 
SLBP was identified in 1996 by the group of W.F. Marzluff as a RNA binding protein that is required for histone pre-mRNA processing (Wang et al., 1996). Already in 1989 a factor that recognizes the stem-loop was described and termed the hairpin binding factor (HBF) by that time (Vasserot et al., 1989). For its high affinity binding the entire sequence of the stem-loop structure and the five flanking nucleotides are required (Williams and Marzluff, 1995). Its role during processing is to stabilize the binding of the U7 snRNP to the HDE and to recruit the cleavage factor to the cleavage site (Dominski and Marzluff, 2007).

The U7 snRNP was the first trans-acting factor identified in replicationdependent histone 3' end processing (Birnstiel and Schaufele, 1988). Its unstructured 5' terminus base pairs with the HDE while its 3' end is folded into a stem-loop. The central portion of the U7 snRNA contains an Sm site around which the Sm proteins assembly and form a ring. This ring consists of seven Sm proteins in which the Sm-like proteins LSM10 and LSM11 replace SmD1 and D2 in the canonical heptameric Sm protein ring that binds spliceosomal snRNAs.

In addition to SLBP and the U7 snRNP, further proteins are crucial to stabilize the processing complex. The heat-labile processing factor Symplekin for example is thought to function as a platform that recruits the cleavage and polyadenylation specificity factors CPSF73 and CPSF100 (Takagaki and Manley, 2000). Recently, Symplekin was identified as a component of the heat-sensitive protein complex that includes all five subunits of CPSF and two subunits of CSTF (Kolev and Steitz, 2005). A further component of the processing machinery of replication-dependent histone mRNAs is the zinc finger protein ZFP100 that is part of the active U7 snRNP and binds to LSM11 and interacts with the SLBP/pre-mRNA complex to help stabilize the binding of the U7 snRNP to the histone pre-mRNA (Azzouz et al., 2005; Dominski et al., 2002; McGinty et al., 2008; Pillai et al., 2003; Wagner and Marzluff, 2006).

Surprisingly, in addition to proteins like SLBP, U7 snRNP and ZFP100 that are unique for the 3' end processing of canonical histone mRNAs, there are also proteins that are common with the processing machinery of polyadenylated mRNAs like Symplekin, CPSF100, CPSF73 and factor interacting with poly(A) polymerase 1 (FIP1) suggesting an evolutionary conservation of the mechanisms for formation of 3 ' ends of all RNAPII transcripts (Marzluff et al., 2008). 


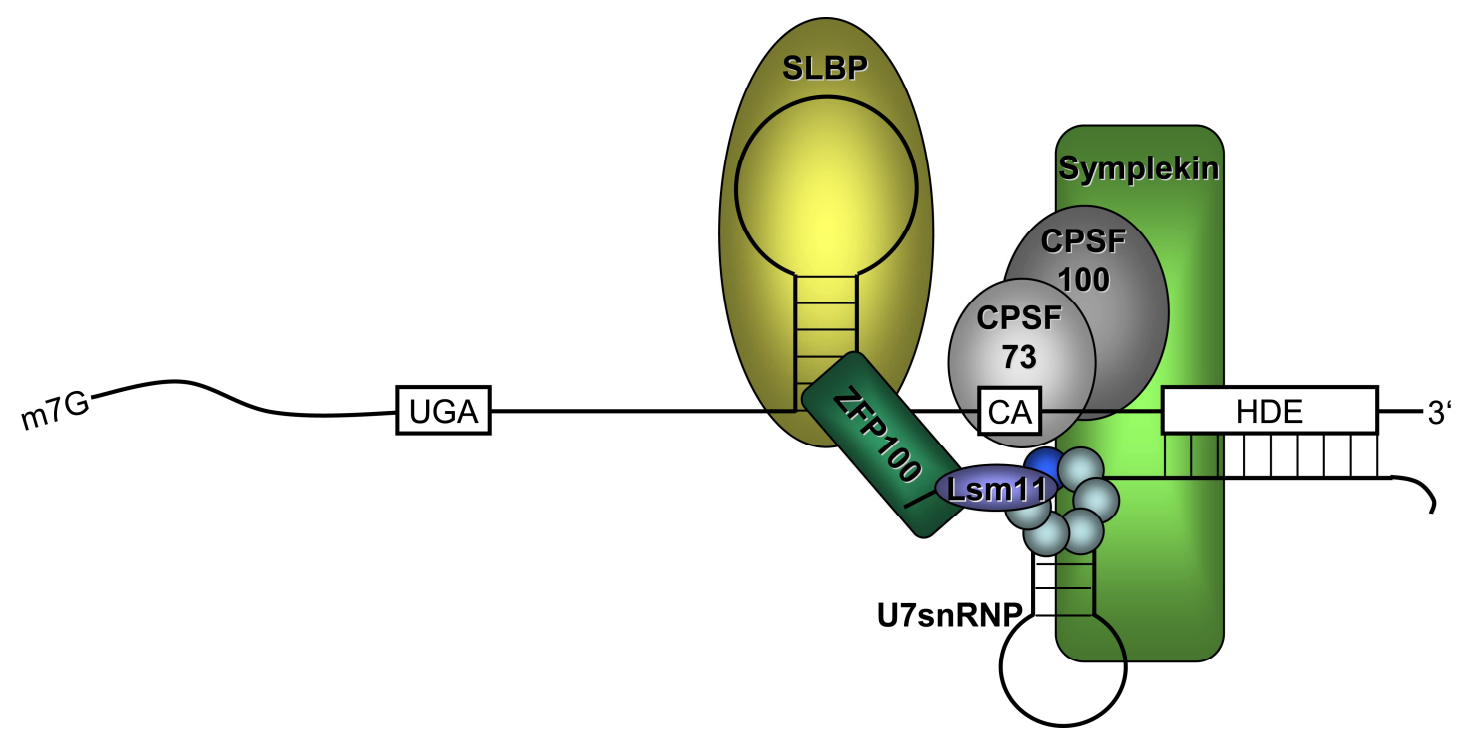

Fig 4: Formation of the processing complex on canonical histone pre-mRNAs. SLBP binds to the stem-loop and U7 snRNA as part of the U7 snRNP base pairs with the HDE. Together these factors recruit a cleavage complex consisting of the endonucleases CPSF73 and CPSF100, Symplekin and other molecules not shown. ZFP100 stabilizes the binding of U7 snRNP by interacting with SLBP and LSM11 (modified from Marzluff et al., 2008).

\subsubsection{Transport, translation and degradation of histone mRNA}

After transcription and cleavage of the histone pre-mRNA 3' end SLBP stays bound to the stem-loop structure and accompanies the mature histone mRNA to the cytoplasm (Whitfield et al., 2004). There it stimulates its translation by acting together with the SLBP-interacting protein 1 (SLIP1) and the eukaryotic translation initiation factor 4- $\gamma$ (EIF4G) to circularize the mature histone mRNA (Cakmakci et al., 2008; Marzluff et al., 2008). Like that the 3' end of the histone mRNA comes into proximity to the 5' cap which increases efficient translation. At the end of S phase or when DNA replication is inhibited histone mRNAs are rapidly degraded in a process that requires SLBP as a cis-element (Pandey and Marzluff, 1987). SLBP recruits the proteins that are crucial for adding a short oligo(U) tail to the histone mRNA like the up-frameshift protein 1 (UPF1) which is a RNA helicase and the terminal uridyltransferase (TUTase) (Kaygun and Marzluff, 2005). The oligo(U) tail then serves as a binding site for the LSM1-7 ring which plays a role in the recruitment of the decapping complex (DCP1DCP2) and the exosome (Parker and Song, 2004; Sullivan et al., 2009). Finally, the histone mRNA is degraded from both the 5' and 3' ends by exonucleases.

SLBP expression and phosphorylation, leading to its degradation, is cell cycledependent and its expression pattern reflects that of histones (Narita et al., 2007; Zheng et al., 2003a). SLBP accumulates just before entry into S phase, is rapidly degraded in 
the end of S phase and is bound to histone mRNA throughout its lifetime (Marzluff et al., 2008). However, there are different molecular signals that regulate both since degradation of histone mRNAs by DNA synthesis inhibitors does not effect SLBP levels nor does stabilization of SLBP at the end of S phase affect histone mRNA degradation (Whitfield et al., 2004; Zheng et al., 2003a) in Marzluff et al., 2008).

\subsubsection{Regulation of replication-dependent histone gene transcription}

The nuclear protein Ataxia Telangiectasia locus (NPAT) was shown to be essential for the entry of cells into $S$ phase and to stimulate histone gene transcription (Ma et al., 2000; Wei et al., 2003; Ye et al., 2003). NPAT was first identified and described in 1996 as being produced from a housekeeping gene positioned close to the Ataxia Telangiectasia locus ( $0.5 \mathrm{~kb}$ from the 5 ' end of ATM) on human chromosome 11q22-q23 (Imai et al., 1996). The protein consists of 1427 amino acids, has an apparent molecular mass of $220 \mathrm{kD}$ and contains a nuclear localization signal and five target sites for phosphorylation by cyclin-dependent protein kinases (Imai et al., 1996; Zhao et al., 1998). NPAT is associated with the histone gene clusters on chromosome $1 \mathrm{q} 21$ and $6 \mathrm{p} 21$ in vivo during $\mathrm{S}$ phase when these are actively expressed and overexpression of NPAT leads to activation of histone H2B and histone H4 transcription (Ma et al., 2000; Zhao et al., 2000; Zheng et al., 2003b). In contrast, inhibition of NPAT expression was shown to lead to a considerable decrease in replication-dependent histone transcripts in human somatic cells (Gao et al., 2003; Ye et al., 2003). The expression of NPAT is cell cycle regulated by the transcription factor E2F whose family members (activator E2F1 and repressor E2F4) can bind to E2F binding sites in the promoter of the NPAT gene resulting in a maximal expression of NPAT at the $\mathrm{G}_{1} / \mathrm{S}$ phase boundary (Gao et al., 2003; Zhao et al., 1998). Its activity is also dependent upon post-translational modifications since it interacts with Cyclin E/CDK2 in vivo through a cyclin-binding RXL motif and is a phosphorylation target of this CDK (Zhao et al., 1998). This phosphorylation is crucial for the colocalization of NPAT with Cyclin E to Cajal bodies during S phase and regulates the ability of NPAT to activate histone gene expression (Bongiorno-Borbone et al., 2008; Ma et al., 2000). Thus, NPAT activity is increased when cells enter $\mathrm{S}$ phase and this in turn leads to a coordinated upregulation of histone gene expression at this stage of the cell cycle (Zhao, 2004). NPAT functions together with proteins that regulate the expression of histone H2B and histone H4 (Mitra et al., 2003; Zheng et al., 2003b). The octamer-binding 
protein 1 (OCT1) coactivator in $\mathrm{S}$ phase (OCA-S) was identified to be essential for $\mathrm{S}$ phase-dependent histone $\mathrm{H} 2 \mathrm{~B}$ transcription. By directly binding to OCT1 it is recruited to histone $\mathrm{H} 2 \mathrm{~B}$ promoters in vitro and in vivo during $\mathrm{S}$ phase (Zheng et al., 2003b). Furthermore, the histone H4 transcription factor (HINFP) was shown to regulate histone $\mathrm{H} 4$ gene expression by interacting with conserved $\mathrm{H} 4$ cell cycle regulatory sequences in vivo utilizing NPAT as a key coactivator to enhance the transcription of the histone $\mathrm{H} 4$ genes (Mitra et al., 2003).

Taking these factors into account, the following model of histone gene regulation by NPAT was recently built. At the $\mathrm{G}_{1} / \mathrm{S}$ boundary NPAT is phosphorylated by Cyclin E/CDK2 which leads to the association of NPAT with histone promoters. This is suggested to occur through its interaction with histone subtype specific binding proteins (SSBPs) like OCT1/OCA-S and HINFP that bind to subtype specific regulatory elements (SSREs) of the respective histone promoters. NPAT then recruits a histone acetyltransferase (HAT) like Tat interacting protein, $60 \mathrm{kDa}$ (TIP60) by binding to a transformation/transcativation domain-associated protein (TRRAP) which interacts with TIP60 resulting in the acetylation and thus activation of the histone promoter (DeRan et al., 2008) (illustrated in Fig 5 on page 28).

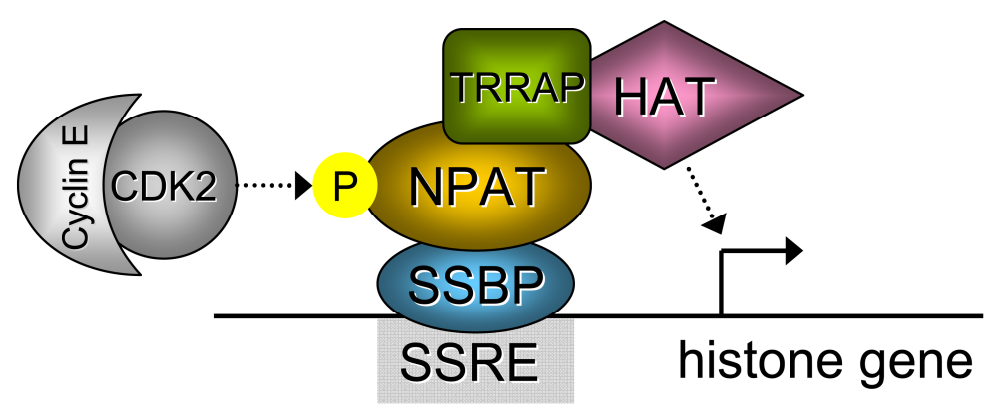

Fig 5: Model for the function of NPAT in activating histone genes. Subtype specific binding proteins (SSBP) bind to subtype specific regulatory elements (SSRE) and associate with NPAT which is then phosphorylated by Cyclin E/CDK2. This leads to the recruitment of a transformation/transcativation domain-associated protein (TRRAP) that interacts with a histone acetyltransferase (HAT) resulting in acetylation and thus activation of histone promoters (modified from DeRan et al., 2008).

\subsubsection{The existence of polyadenylated replication-dependent histone mRNAs}

Polyadenylated mRNAs produced from replication-dependent histone genes were identified that in many cases still contain the characteristic sequences for canonical histone 3' end processing (Davila and Samuelsson, 2008). This suggests that under certain circumstances transcripts produced from some histone genes may be either processed by polyadenylation or by the histone-specific mechanism. Interestingly, the 
loss of normal histone mRNA processing by depletion of SLBP or U7 snRNP can result in the production of polyadenylated histone mRNAs (Sullivan et al., 2001; Wagner et al., 2007). The same effect was observed by knocking down the CDK9 target NELF-E or a component of the cap binding complex (CBC), the cap binding protein 80 (CBP80), giving evidence that some histone mRNAs can be processed in both ways and thus two processing pathways exist (Narita et al., 2007).

The histone genes mainly used in our studies, HIST1H2BD and HISTIH2AC, produce besides normally processed transcripts also polyadenylated transcripts resulting from the splicing of a longer primary transcript. Interestingly, polyadenylated histone transcripts are found in the human EST database for a number of replication-dependent histone genes. Since both genes, HIST1H2BD and HIST1H2AC, can be spliced, as an example of a non-spliced replication-dependent histone transcript that can be polyadenylated, the HIST2H2AA gene was chosen, that is part of the HIST2 cluster located on chromosome 1 (Marzluff et al., 2002).

Studies have shown that the levels of polyadenylated canonical histone transcripts are very low in proliferating cells but may increase during differentiation or tumorigenesis (Abba et al., 2005; Collart et al., 1991; Kirsh et al., 1989; Martinez et al., 2007; Narita et al., 2007; Zhao et al., 2004). These observations lead to the speculation that these polyadenylated histone transcripts produced from (normally) replicationdependent histone genes may serve a physiological role.

\subsubsection{The tumor suppressor p53 regulates cell cycle progression through p21}

P53 was first described in 1979 as a $53 \mathrm{kDa}$ protein which was shown to have tumor suppressor function (Baker et al., 1989; DeLeo et al., 1979; Finlay et al., 1989; Lane and Crawford, 1979; Linzer and Levine, 1979). P53 is a transcription factor that is activated upon various stress signals resulting in several cellular responses including cell cycle arrest, senescence and apoptosis (Polager and Ginsberg, 2009). The p53 gene is mutated in approximately $50 \%$ of all human tumors pointing out the importance of proper p53 function for normal cell growth (Hollstein et al., 1991). Upon activation p53 can directly bind as a tetramer to specific sequences called p53 responsive elements in the promoter region of target genes and activate their transcription. For example, p53 can induce a $G_{1}$ cell cycle arrest by directly stimulating the expression of the $p 21$ cyclin-dependent kinase inhibitor gene (DelSal et al., 1996). 
The $p 21$ gene is localized to chromosome 6p21.2 and was identified in 1993 as being directly induced by p53 (El-Deiry et al., 1992). The encoded protein belongs to the Cip and Kip family of CDK inhibitors including p21, p27 and p57 which decrease the cell cycle-regulated activity of CDKs, where p21 primarily inhibits Cyclin E/CDK2 activity (Abbas and Dutta, 2009). As already mentioned in section 1.3.1.1 on page 19ff. Cyclin E/CDK2 activity is crucial for inhibition of the retinoblastoma protein via its phosphorylation, meaning that decreased Cyclin E/CDK2 activity following p53induced p21 gene expression results in decreased phosphorylation of $\mathrm{RB}$ and consequently decreased activity of the E2F transcription factor (illustrated in Fig 6 on page 31$)$.

\subsubsection{The p53 regulator MDM2 and its inhibition by Nutlin-3a}

The p53 tumor suppressor is negatively regulated by the MDM2 protein encoded by the murine double minute 2 (MDM2) gene (Iwakuma and Lozano, 2003). Via direct association, MDM2 alters the subcellular organization of p53, its stability and its ability to function as a transcriptional activator (Freedman et al., 1999; Onel and CordonCardo, 2004). Being a RING finger-containing ubiquitin protein ligase, the $\mathrm{N}$-terminus of MDM2 can bind to p53 and target it for ubiquitination and thus degradation by the proteasome (Fang et al., 2000; Iwakuma and Lozano, 2003). The MDM2 gene itself is a target gene of 553 and by binding to its promoter, p53 upregulates its expression. Thus, accumulated MDM2 can then bind to p53 and thus serve as a negative feedback mechanism. A decrease in p53 levels in turn leads to decreased levels of MDM2 expression. Thus, a tightly regulated feedback loop is formed that controls the function of p53 and MDM2. Furthermore, MDM2 can also ubiquitinate itself which leads to self degradation and is another example for regulating p53 activity (Fang et al., 2000 and Iwakuma and Lozano, 2003). Thus, in cancer cells and tumors that are wild type for p53, the inhibition of MDM2 by chemical compounds has become a popular strategy to reactivate p53 activity (Vassilev, 2007).

In 2004, Vassilev et al. developed a cis-imidazoline analog that inhibits the interaction of p53 and MDM2, named Nutlin (for Nutley inhibitor). This inhibitor binds to a deep hydrophobic pocket of the MDM2 protein which is normally occupied by three side chains of the helical region of $\mathrm{p} 53, \mathrm{Phe}^{19}, \operatorname{Trp}^{23}$ and Leu ${ }^{26}$ (Kussie et al., 1996; Vassilev et al., 2004). This interaction surface is essential for p53-MDM2 interactions and the ubiquitination and subsequent degradation of p53 by MDM2. By 
mimicking these three amino acids and their orientation Nutlin can impair the binding of p53 to MDM2. Thus, p53 is accumulated and stabilized in the cell and its transcriptional activity is increased which results in cell cycle arrest or apoptosis (Eischen and Lozano, 2009). Besides Nutlin and further agents that impede the association of p53 with MDM2, compounds that inhibit the ubiquitin ligase activity of MDM2 are in preclinical development as well (Vazquez et al., 2008).

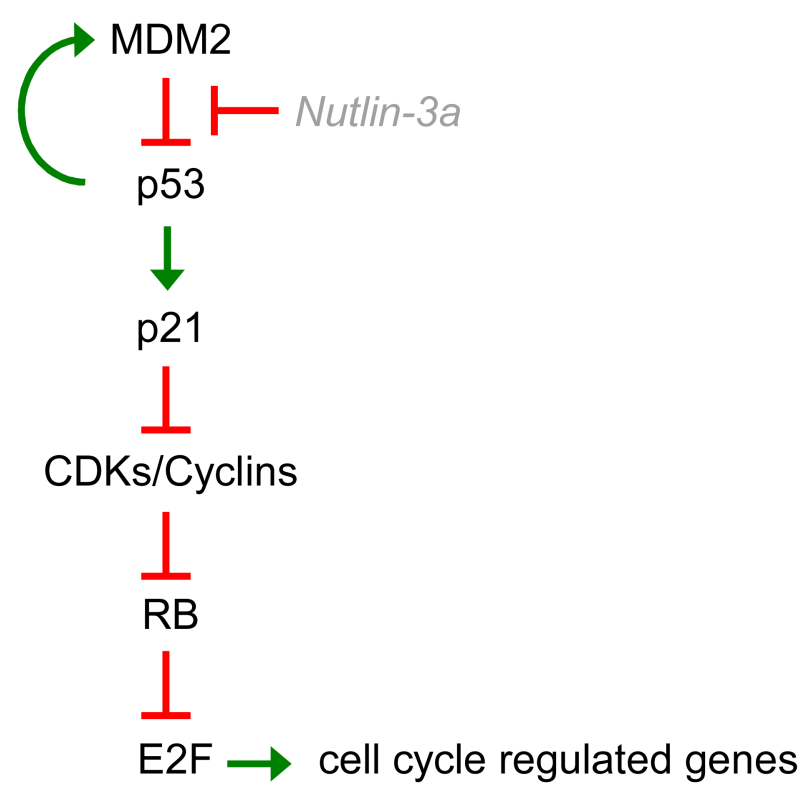

Fig 6: The p53/RB/E2F pathway. The accumulation of p53 (e.g., by inhibiting MDM2 activity with Nutlin-3a) increases $p 21$ gene transcription, thereby decreasing the activity of CDKs/Cyclins. Thus, RB phosphorylation is impeded which leads to its activity and complex formation with E2F transcription factors. Finally, the inhibition of E2Fs results in inhibition of the transcription of cell cycle regulated genes.

\subsubsection{Does p53 regulation play a role in the expression of polyadenylated replication-dependent histone mRNAs?}

Microarray studies, that are typically based on an initial polyT-primed reverse transcription step and thus specifically label polyadenylated transcripts, identified changes in the expression of replication-dependent histone genes. Interestingly, a correlation between replication-dependent histone mRNA polyadenylation and p53 status in head and neck squamous cell carcinomas could be observed (Yan et al., 2007). In this study, the expression of polyadenylated replication-dependent histone mRNAs were increased in tumors that contain wild type p53 in comparison to normal human keratinocytes and they were expressed in an even higher level in tumors that contain mutant p53. Similarly, another study identified a change in the expression of 
polyadenylated replication-dependent histone mRNAs in human papilloma virus positive $(\mathrm{HPV}+)$ squamous head and neck cell carcinomas compared to HPV-tumors (Martinez et al., 2007). The HPV E6 oncoprotein is a primary target of p53 meaning that the observations could be due to regulation of p53 function (zur Hausen and de Villiers, 1994). 


\section{Publications}

This thesis is based on the following original publications.

\section{Publication I:}

Pirngruber, J., Shchebet, A., Schreiber, L., Shema, E., Minsky, N., Chapman, R.D., Eick, D., Aylon, Y., Oren, M., and Johnsen, S.A. (2009). CDK9 directs H2B monoubiquitination and controls replication-dependent histone mRNA 3' end processing. EMBO Rep. 10, 894-900.

Publication II:

Pirngruber, J., Shchebet, A., and Johnsen, S.A. (2009). Insights into the function of the human $\mathrm{P}-\mathrm{TEFb}$ component $\mathrm{CDK} 9$ in the regulation of chromatin modifications and co-transcriptional mRNA processing. Cell Cycle 8, 3636-3642.

\section{Publication III:}

Pirngruber, J. and Johnsen, S.A. (2010). Induced $\mathrm{G}_{1}$ cell cycle arrest controls replication-dependent histone mRNA 3' end processing through p21, NPAT and CDK9. Oncogene, Epub ahead of print.

The spelling of the publication part refers to the original articles and may thus differ from the spelling used in the other parts of this thesis. 


\subsection{Publication I}

\section{CDK9 directs H2B monoubiquitination and controls replication-dependent histone mRNA 3' end processing}

\section{Citation}

Judith Pirngruber, Andrei Shchebet, Lisa Schreiber, Efrat Shema, Neri Minsky, Rob D. Chapman, Dirk Eick, Yael Aylon, Moshe Oren and Steven A. Johnsen

CDK9 directs H2B monoubiquitination and controls replication-dependent histone mRNA 3' end processing

EMBO Reports (2009), 10(8): 894-900

doi:10.1038/embor.2009.108

\section{Own contribution}

Preparation and accomplishment of the biological experiments presented in Fig I.1A, Fig I.2 (except for D), Fig I.3, Fig I.4, Supp Fig I.S1 (except for E) and Supp Fig I.2, i.e. inhibitor treatments, transfection of described siRNAs and constructs, western blot analysis, gene expression analysis, chromatin immunoprecipitation (ChIP), real-time PCR; complete figure layout, tables, supplemental materials and methods; contribution to the writing of the manuscript (parts of introduction, methods and results section). 


\begin{abstract}
Post-translational histone modifications have essential roles in controlling nuclear processes; however, the specific mechanisms regulating these modifications and their combinatorial activities remain elusive. Cyclin-dependent kinase 9 (CDK9) regulates gene expression by phosphorylating transcriptional regulatory proteins, including the RNA polymerase II carboxy-terminal domain. Here, we show that CDK9 activity is essential for maintaining global and gene-associated levels of histone H2B monoubiquitination (H2Bub1). Furthermore, CDK9 activity and H2Bub1 help to maintaining proper replication-dependent histone messenger RNA (mRNA) 3'-end processing. CDK9 knockdown consistently resulted in inefficient recognition of the correct mRNA 3'-end cleavage site and led to increased read-through of RNA polymerase II to an alternative downstream polyadenylation signal. Thus, CDK9 acts to integrate phosphorylation during transcription with chromatin modifications to control co-transcriptional histone mRNA processing.
\end{abstract}

\title{
Introduction
}

Covalent histone modifications regulate the chromatin environment and are essential for correct transcriptional regulation (Berger, 2007). Histone H3 Lys4 trimethylation ( $\mathrm{H} 3 \mathrm{~K} 4 \mathrm{me} 3)$ is associated with active gene promoters, whereas histone $\mathrm{H} 2 \mathrm{~B}$ monoubiquitination ( $\mathrm{H} 2 \mathrm{Bub} 1)$ is found in the transcribed regions of active genes (Berger, 2007; Weake and Workman, 2008). Genome-wide analyses of histone modifications have shown that the regulation of gene expression is more complex than initially thought, and revealed extensive cross talk between chromatin modifications and the transcriptional machinery (Egloff and Murphy, 2008; Suganuma and Workman, 2008).

$\mathrm{H} 2 \mathrm{Bub} 1$ is carried out by the E3 ubiquitin ligase complex RNF (ring finger) 20/40 in humans (Kim et al., 2005a; Zhu et al., 2005b). RNF20 has tumor suppressor activity as loss of its expression results in a tumorigenic and more invasive phenotype (Shema et al., 2008). Surprisingly, although H2Bub1 is present on most active genes in humans, only a subset of genes actually requires H2Bub1 for expression (Minsky et al., 2008; Shema et al., 2008). Thus, further nuclear functions might require H2Bub1 for their activity.

The RNA polymerase II (RNAPII) carboxy-terminal domain (CTD) contains a conserved heptapeptide repeat that occurs 52 times in humans (Egloff and Murphy, 
2008). Ser5 of the repeat is phosphorylated at the promoter, and is associated with messenger RNA (mRNA) capping and the onset of transcription elongation. Ser2 (PSer2) is phosphorylated by cyclin-dependent kinase 9 (CDK9) in the transcribed region and is required for post-initiation transcription events such as elongation, splicing and mRNA processing. CDK9 further stimulates elongation by phosphorylating negative elongation factor-E (NELF-E) and suppressor of Ty homologue 5 (SUPT5H). Similar to $\mathrm{P}-\mathrm{Ser} 2, \mathrm{H} 2 \mathrm{Bub} 1$ is enriched in the transcribed region of actively transcribed genes (Minsky et al., 2008). Although P-Ser2 and H2Bub1 are disconnected in yeast, a link in humans has not been investigated (Wood et al., 2007; Xiao et al., 2005).

Unlike most mRNAs, replication-dependent histone transcripts end in a conserved stem-loop recognized by the stem-loop binding protein (SLBP) and cleaved by the U7 small nuclear ribonucleoprotein (snRNP; (Marzluff et al., 2008). Loss of correct histone mRNA processing by the depletion of SLBP or U7 increases the formation of polyadenylated histone transcripts (Sullivan et al., 2001; Wagner et al., 2007). Replication-dependent histone genes have been identified as targets of both H2Bub1 (RNF20) and the CDK9 target NELF-E (Narita et al., 2007; Shema et al., 2008). Although CDK9 activity has been linked to the transcription and processing of polyadenylated mRNAs, its role in histone mRNA 3'-end processing remains unknown. Furthermore, the contribution of specific histone modifications to mRNA processing is also unknown. Here, we report that CDK9 regulates H2Bub1 through a CTD-dependent mechanism involving the RNA polymerase II associated factor (PAF)-RNF20/40 complex. Furthermore, CDK9 and H2Bub1 are required for correct replicationdependent histone mRNA 3'-end processing, and together reveal a new role for CTD phosphorylation and chromatin modifications in controlling this process.

\section{Results and Discussion}

\section{Transcription is not sufficient for maintaining H2Bub1}

As H2Bub1 is associated with the transcribed regions of active genes, we tested whether transcription is essential for maintaining global levels of H2Bub1. Consistent with previous studies (Davie and Murphy, 1990; Minsky et al., 2008), actinomycin D rapidly decreases global levels of $\mathrm{H} 2 \mathrm{Bub} 1$, confirming that active transcription is necessary for maintaining $\mathrm{H} 2 \mathrm{Bub} 1$ and showing the rapid dynamics of $\mathrm{H} 2 \mathrm{~B}$ deubiquitination (Fig I.1A). 

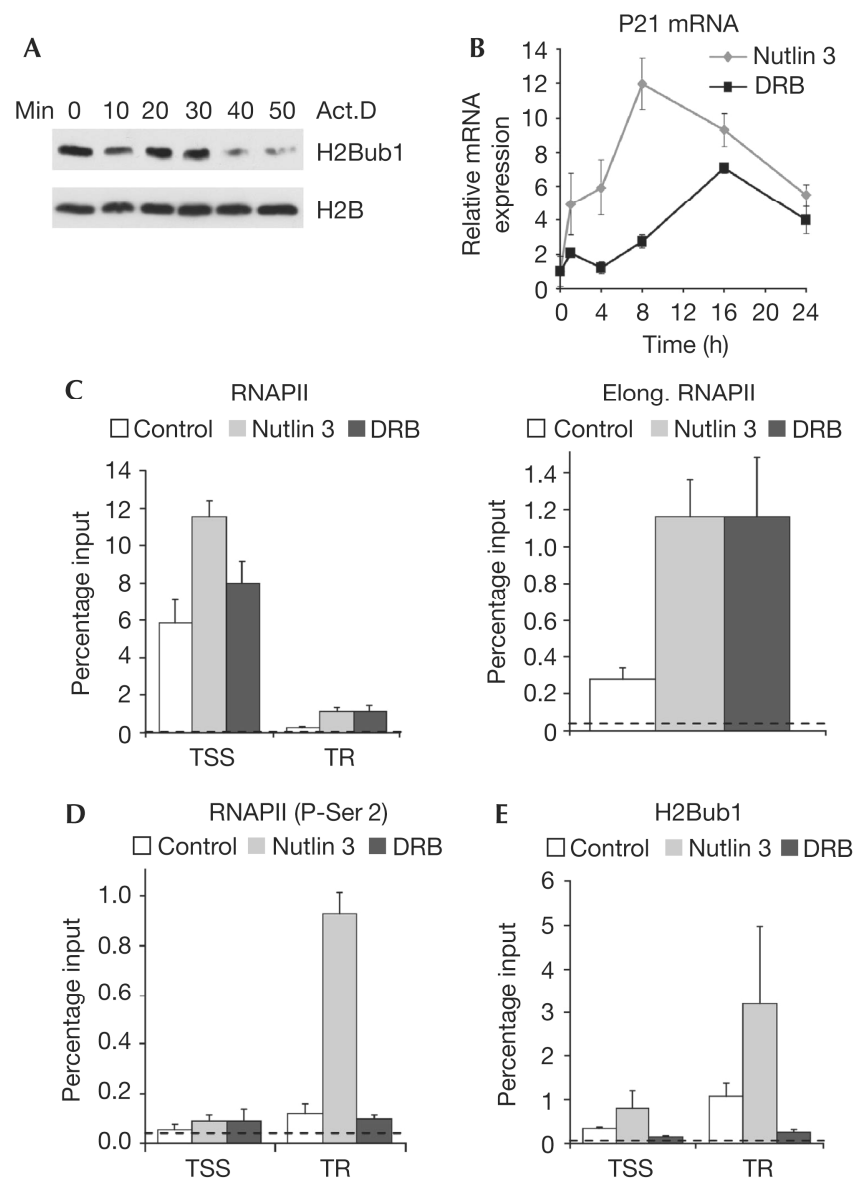

Fig I.1: H2Bub1 depends on CDK9 activity rather than transcriptional activation per se. (A) H1299 cells were treated with actinomycin $\mathrm{D}$ for the indicated times to inhibit transcription, and protein lysates were analysed by Western blot for H2Bub1 and H2B protein levels. (B) Nutlin 3 and DRB both increase levels of p21 mRNA. U-2 OS cells were treated with $8 \mu \mathrm{M}$ nutlin 3 or $50 \mu \mathrm{M}$ DRB for the indicated times before RT-PCR analyses. Transcript levels were normalized to mitochondrial 16S ribosomal RNA and expressed relative to the control treatment; mean values + s.d., $n=3$. (C-E) P-Ser 2, rather than elongation by RNAPII, is essential for maintaining gene-associated H2Bub1. U-2 OS cells were treated with nutlin 3 or DRB for $8 \mathrm{~h}$ and analysed by ChIP using antibodies against (C) total RNAPII, (D) P-Ser2 or (E) H2Bub1. qChIP values were normalized to their respective DNA inputs and expressed as DNA recovery (percentage input); mean values + s.d., $n=3$. The experimental background is shown as a dotted line. Act.D, actinomycin D; CDK9, cyclin-dependent kinase 9; ChIP, chromatin immunoprecipitation; DRB, 5,6-dichloro- $\beta$-D-ribofuranosyl benzimidazole; H2Bub1, histone H2B monoubiquitination; mRNA, messenger RNA; qChIP, quantitative chromatin immunoprecipitation; RNAPII, RNA polymerase II; RTPCR, reverse transcription PCR; TR, transcribed region; TSS, transcriptional start site.

To test whether active transcription is sufficient for H2Bub1 accumulation, we exploited the $\mathrm{p} 53$ response gene $p 21$, the transcription of which is uniquely induced by 5,6-dichloro- $\beta$-D-ribofuranosyl benzimidazole (DRB) despite a loss of P-Ser2 (Gomes et al., 2006). p21 mRNA levels significantly increased following treatment with either DRB or the specific mouse double minute 2 (MDM2) inhibitor nutlin 3 (Fig I.1B) and correlated with an increase in elongation of RNAPII on the $p 21$ gene (Fig I.1C), as detected by chromatin immunoprecipitation (ChIP). As reported previously, the levels of RNAPII at the transcriptional start site (TSS) were only modestly affected by either 
treatment, whereas both P-Ser2 (Gomes et al., 2006) and H2Bub1 (Minsky et al., 2008) specifically increased in the transcribed region (Fig I.1D,E) following nutlin 3 treatment. In contrast to total RNAPII, P-Ser2 and H2Bub1 levels did not increase in the transcribed region after DRB treatment (Fig I.1D,E).

\section{CDK9 activity maintains global H2Bub1 levels}

As $\mathrm{H} 2 \mathrm{Bub} 1$ and P-Ser2 are primarily present in the transcribed regions of active genes, we hypothesized that CDK9 is essential for H2Bub1. Therefore, we treated cells with four chemical CDK9 inhibitors and analysed total levels of H2Bub1. Each significantly decreased both H2Bub1 and P-Ser2 (Fig I.2A). These effects were dosedependent (Supp Fig I.S1A; data not shown) and specific, as the levels of H2B and PSer5 were unaltered (Fig I.2A); RNF20/40 protein levels were also unaffected (Supp Fig I.S1B).

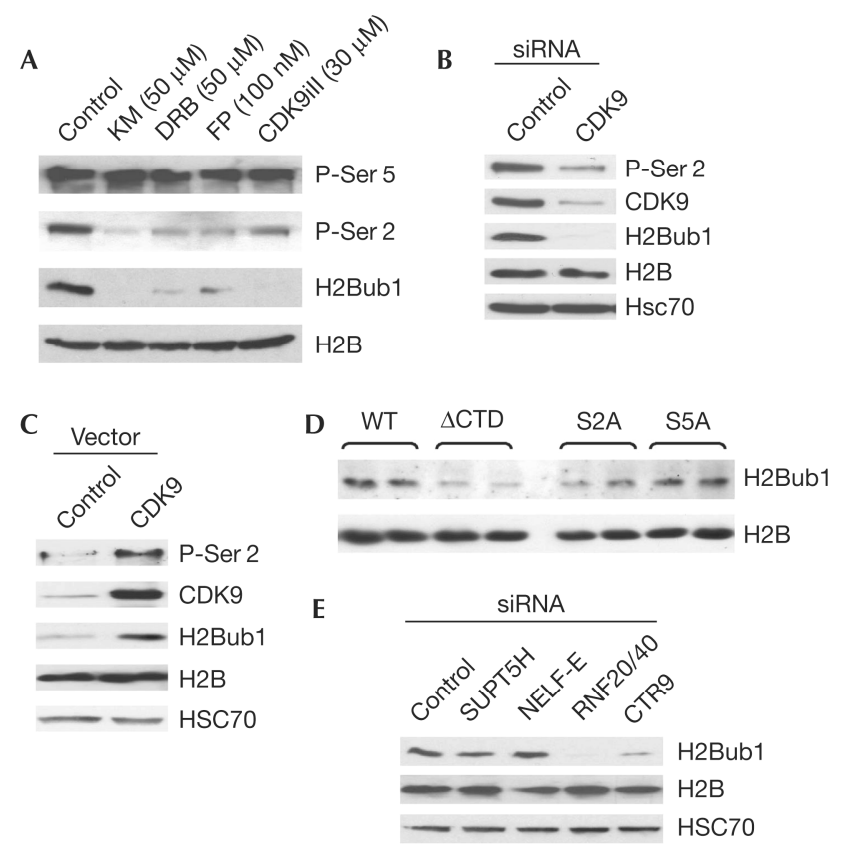

Fig I.2: CDK9 activity is essential for maintaining global levels of H2Bub1. (A) H1299 cells were treated with the specific CDK9 inhibitors KM, DRB, FP or CDK9 inhibitor II (CDK9iII) at the indicated concentrations for $4 \mathrm{~h}$ and analysed by Western blot analysis. (B,C) H1299 cells were transfected with (B) control or CDK9 siRNAs or (C) expression plasmids and analysed by Western blot with the indicated antibodies. (D) The CTD and Ser 2 are necessary for maintaining H2Bub1 levels. HEK293 cells were transfected with $\alpha$-amanitin-resistant RNAPII large subunit expression constructs containing wild-type (WT), deletion or mutant CTD. (E) Regulation of H2Bub1 by SUPT5H, NELF-E, RNF20/40 and PAF1. H1299 cells were transfected and analysed by Western blot using the indicated siRNAs and antibodies. H2B and HSC70 are shown as loading controls. CDK9, cyclin-dependent kinase 9; CTD, carboxyterminal domain; DRB, 5,6-dichloro- $\beta$-D-ribofuranosyl benzimidazole; FP, flavopiridol; H2Bub1, histone H2B monoubiquitination; HEK, human embryonic kidney; HSC70, heat shock cognate protein 70; KM, KM05283; NELF-E, negative elongation factor E; PAF, polymerase II associated factor; RNAPII, RNA polymerase II; RNF, ring finger protein; siRNA, small interfering RNA; SUPT5H, suppressor of Ty 5 homologue. 
To verify further the role of CDK9, we transfected cells with specific small interfering RNAs (siRNAs) against CDK9. These decreased the level of both P-Ser2 and H2Bub1 (Fig I.2B; Supp Fig I.S1C). This effect was rescued by re-expressing the mouse Cdk9 (Supp Fig I.S1D) and the overexpression of CDK9 alone increased the level of both P-Ser2 and H2Bub1 (Fig I.2C).

To determine whether CTD phosphorylation is required for $\mathrm{H} 2 \mathrm{Bub} 1$, we transfected cells with $\alpha$-amanitin-resistant RNAPII constructs with wild-type CTD, no CTD, or CTD with specific mutations at Ser2 or Ser5. Both an intact CTD and Ser2, but not Ser5, were essential for H2Bub1 (Fig I.2D; Supp Fig I.S1E). Knockdown of SUPT5H or NELF-E had little effect on global levels of H2Bub1 (Fig I.2E). Thus, CDK9 affects H2Bub1 primarily in a CTD-dependent manner. Consistent with a previous report (Zhu et al., 2005b), we also observed a decrease in H2Bub1 after knockdown of the PAF component CTR9 (Fig I.2E).

\section{RNF40 is essential for maintaining global H2Bub1 levels}

Conflicting reports have left the role of RNF40 in H2Bub1 unclear (Kim et al., 2005a; Weake and Workman, 2008; Zhu et al., 2005b). Thus, we tested whether both RNF20 and RNF40 regulated H2Bub1. RNF40 knockdown decreased H2Bub1, and this effect was rescued by overexpression of mouse Rnf40 (Supp Fig I.S1F). Similarly, the knockdown of either RNF20 or RNF40 using multiple siRNAs decreased global levels of H2Bub1 (Supp Fig I.S1G,H).

\section{CDK9 and RNF20/40 control histone mRNA processing}

The CDK9 dependence of H2Bub1 suggested that these molecules might influence the same nuclear processes. As histone genes are targets of both H2Bub1 (RNF20) and NELF-E (Narita et al., 2007; Shema et al., 2008), we tested whether CDK9 or RNF20/40 control replication-dependent histone gene transcription. However, none of these molecules significantly affected the mRNA levels of HIST1H2BD, HIST1H2AC or HIST2H2AA (Fig I.3B and Supp Fig I.S2A).

Replication-dependent histone mRNAs are not usually polyadenylated, but instead end in a conserved stem-loop structure. As knockdown of the CDK9-target NELF-E increased replication-dependent histone mRNA polyadenylation (Narita et al., 2007), we tested whether CDK9, RNF20 or RNF40 also influences replicationdependent histone mRNA 3'-end processing. The HIST1H2BD and HIST1H2AC genes 
produce not only mRNAs containing 3'-end stem-loop structures but also the polyadenylated forms (Fig I.3A); their size - 13 and $15 \mathrm{~kb}$, respectively - also make them amenable to ChIP analyses. Indeed, CDK9 knockdown increased read-through of the HISTIH2BD gene approximately 15-fold (Fig I.3C); the increased read-through transcript was also polyadenylated (Fig I.3D). The magnitudes of these effects were similar to those observed after NELF-E or SLBP knockdown (Narita et al., 2007). The knockdown of RNF20, RNF40, CTR9, cyclinT1, SUPT5H or NELF-E also resulted in increased HIST1H2BD polyadenylation (Fig I.3D and Supp Fig I.S2B). The modest effects of RNF20 and RNF40 knockdown probably reflect the pleiotropic effects of CDK9 knockdown on histone mRNA processing, which probably affects not only the CTD-dependent regulation of H2Bub1 but also the functions of SUPT5H and NELF-E. Similar results were obtained for the HIST1H2AC and HIST2H2AA genes (Fig I.3E and Supp Fig I.S2A). Importantly, these effects were not due to changes in cell-cycle progression, as CDK9, RNF20 or RNF40 knockdown did not affect cell-cycle distribution (data not shown). These effects were specific for replication-dependent histone mRNAs as the levels of polyadenylated 36B4, $\beta$-actin and GAPDH were unaffected by the knockdowns (Supp Fig I.S2C; data not shown). Thus, CDK9 and H2Bub1 help to maintain the correct mode of 3'-end processing of replicationdependent histone mRNAs.

Fig I.3: CDK9 and H2Bub1 direct replication-dependent histone messenger RNA 3'-end processing but not transcription. (A) Schematic representation of the human HIST1H2BD and HIST1H2AC genes showing both normally processed and polyadenylated transcripts resulting from the splicing of a longer transcript. (B) Total HIST1H2BD and HIST1H2AC expression is not significantly affected by CDK9, RNF20 or RNF40 knockdown. Random-primed cDNAs were analysed for total HIST1H2BD or HIST1H2AC expression after transfection of HEK293 cells with control, CDK9, RNF20 or RNF40 siRNAs. The expression of total histone transcripts was normalized to a control gene, 36B4, and is represented as relative mRNA expression; mean values + s.d., $n=3$. (C) CDK9 knockdown increases the formation of read-through HIST1H2BD transcripts. Total RNA from HEK293 cells transfected with control or CDK9 siRNA was analysed by random-primed qRT-PCR using specific primers for total and read-through HIST1H2BD transcripts. The expression of processed (left panel) and read-through transcripts (right panel) was normalized to $36 B 4$ and is shown as the percentage total transcript; mean values + s.d., $n=3$. (D,E) CDK9 and RNF40/20 knockdown increase the formation of (D) polyadenylated HIST1H2BD and (E) HIST1H2AC transcripts. Total mRNA from cells transfected with control, CDK9, RNF40 or RNF20 siRNA was analysed by oligo-dT-primed qRT-PCR. Expression levels were normalized and expressed as in (A); mean values + s.d., $n=3$. CDK9, cyclin-dependent kinase 9; cDNA, complementary DNA; ChIP, chromatin immunoprecipitation; H2Bub1, histone H2B monoubiquitination; HEK, human embryonic kidney; mRNA, messenger RNA; RNF, ring finger protein; siRNAs, small interfering RNAs, qRT-PCR, quantitative reverse transcription PCR; RNF, ring finger protein; siRNAs, small interfering RNAs. 

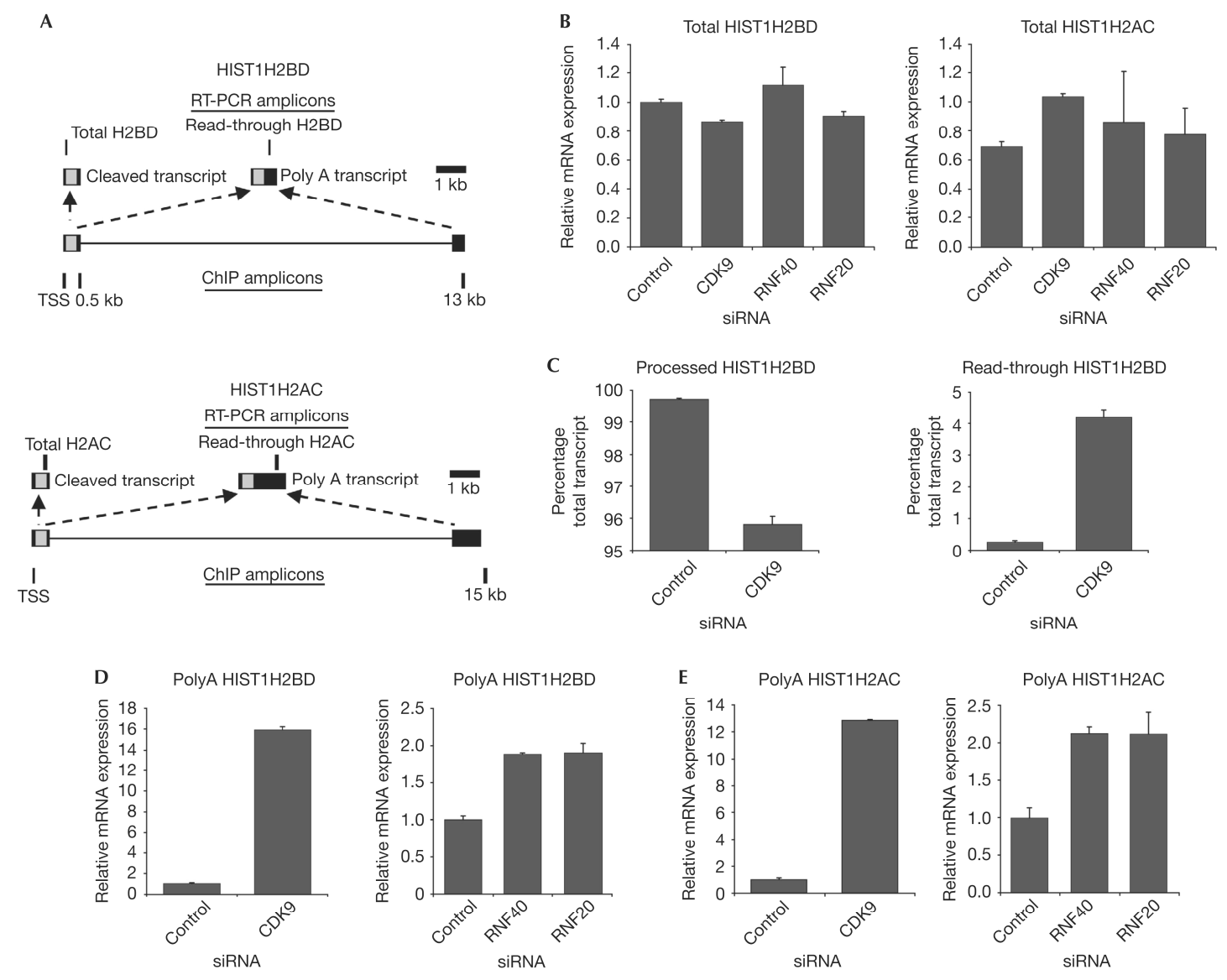

\section{CDK9 regulates RNAPII read-through genes}

Next, we carried out ChIP analyses to test whether CDK9 knockdown affected the recruitment of specific factors to the HISTIH2BD gene. TATA-binding protein (TBP) was recruited preferentially to the TSS and its levels were unchanged by CDK9 knockdown (Fig I.4A), suggesting that the basal transcriptional apparatus was not affected by CDK9 knockdown. The knockdown of CDK9 and its presence on the HIST1H2BD gene were also verified (Fig I.4A). P-Ser2 was consistently decreased to nearly undetectable levels after CDK9 knockdown (Fig I.4B). Surprisingly, the amount of total RNAPII at the TSS was decreased to about 50\%, whereas the levels at the 3'cleavage site $(0.5 \mathrm{~kb})$ remained unchanged (Fig I.4B). In comparison to P-Ser2, the fraction of P-Ser5 RNAPII was only moderately affected at both positions (Fig I.4B; Supp Fig I.2D). A similar effect was observed for the recruitment of NELF-E whereas the recruitment of cap binding protein 80 (CBP80) was unaffected by CDK9 knockdown (Supp Fig I.S2E). 


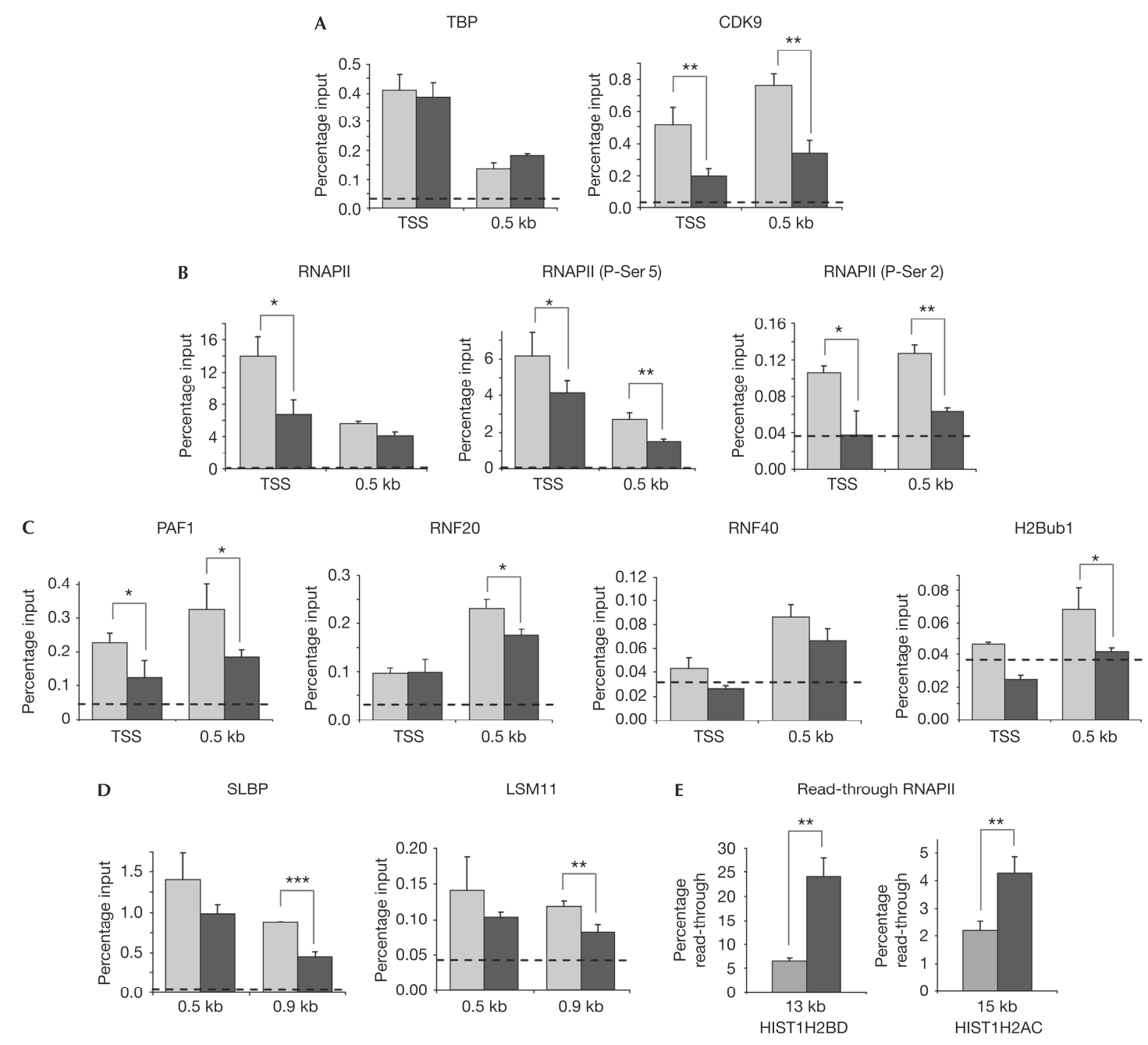

Fig I.4: CDK9 activity recruits proteins involved in H2B ubiquitination and histone messenger RNA 3'-end formation, and decreases RNAPII read-through. ChIP analysis of the HISTIH2BD (or HIST1H2AC in (E)) gene in HEK293 cells after transfection with control (grey bars) or CDK9 (black bars) siRNAs using the indicated antibodies. (A) TBP is specifically enriched at the TSS and is not affected by CDK9 knockdown, whereas CDK9 is significantly enriched both at the TSS and at $0.5 \mathrm{~kb}$ and these levels decrease on knockdown. (B) CDK9 knockdown similarly decreases RNAPII and P-Ser 5RNAPII levels at the TSS without affecting elongation to the 3'-end cleavage site. By contrast, P-Ser 2 is markedly decreased to near-background levels both at the TSS and at $0.5 \mathrm{~kb}$. (C) The H2B-ubiquitinating complex (PAF1, RNF20 and RNF40) is enriched at $0.5 \mathrm{~kb}$ of the HIST1H2BD gene together with H2Bub1, and their levels decrease following CDK9 knockdown. (D) ChIP analysis of SLBP and the U7 snRNP component LSM11 shows decreased recruitment to the 3'-cleavage site. (E) CDK9 knockdown increases RNAPII read-through past the normal 3'-end cleavage site of the HISTIH2BD and HISTIH2AC genes. ChIP analysis of total RNAPII levels at the polyadenylation site (13 kb for HISTIH2BD or $15 \mathrm{~kb}$ for $H I S T 1 H 2 A C$ ) were compared with the amount of total initiating RNAPII (at the TSS) and plotted as the percentage read-through of RNAPII. ChIP experiments are shown as percentage input as in Fig. I.1; mean values + s.d., $n=3$. Statistically significant differences are indicated: $* P \leq 0.05 ; * * P \leq 0.01 ; * * * P$ $\leq 0.001$. The experimental background is shown in each graph as a dotted line. CDK9, cyclin-dependent kinase 9; ChIP, chromatin immunoprecipitation; H2Bub1, histone H2B monoubiquitination; HEK, human embryonic kidney; LSM11, Sm-like protein 11; PAF, RNA polymerase II associated factor; RNAPII, RNA polymerase II; RNF, ring finger protein; SLBP, stem-loop (histone) binding protein; siRNAs, small interfering RNAs; snRNP, small nuclear ribonucleoprotein; TBP, TATA-binding protein; TSS, transcriptional start site, $0.5 \mathrm{~kb}$ is equal to 3 '-end cleavage site, $0.9 \mathrm{~kb}$ is equal to $400 \mathrm{bp} \mathrm{3}$ ' to the cleavage site, $13 \mathrm{~kb}$ or $15 \mathrm{~kb}$ is equal to polyadenylation site of the read-through transcripts for HIST1H2BD and HIST1H2AC, respectively. 
Given that CDK9 was essential for global levels of H2Bub1, and as both CDK9 and $\mathrm{H} 2 \mathrm{Bub} 1$ regulated replication-dependent histone mRNA processing, we tested whether H2Bub1 and the PAF1/RNF20/40 complex were also present on the HIST1H2BD gene. Indeed, RNF20, RNF40, and H2Bub1 showed a preferential enrichment to the 3'-cleavage site, with very low levels at the TSS (Fig I.4C). Importantly, the presence of PAF1 and all of the H2Bub1-associated factors decreased after CDK9 knockdown. This was most apparent for PAF1 and H2Bub1, as PAF1 enrichment at $0.5 \mathrm{~kb}$ was decreased to $50 \%$ above background levels and $\mathrm{H} 2 \mathrm{Bub} 1$ was nearly undetectable following CDK9 knockdown.

The increased polyadenylation of replication-dependent histone mRNAs might be attributable to incorrect recognition of the 3' stem-loop structure, which might result in read-through of RNAPII beyond the normal 3'-processing site. Thus, we carried out ChIP analyses of SLBP and the U7 snRNP component Lsm11 on the HIST1H2BD gene to test whether their recruitment is affected by CDK9 knockdown. Indeed, both SLBP and Lsm11 were enriched at the 3'-cleavage position, as well as $400 \mathrm{bp}$ downstream of the cleavage position, and decreased following CDK9 knockdown (Fig I.4D). Most notably, SLBP recruitment at $0.9 \mathrm{~kb}$ decreased nearly $50 \%$ following CDK9 knockdown.

The decrease in the recruitment of histone mRNA 3'-end processing factors also correlated with an increase in read-through of RNAPII beyond the normal 3'-end cleavage position on the HISTIH2BD gene (Fig I.4E); similar results were obtained for HISTIH2AC (Fig I.4E). This is consistent with the increase in polyadenylated transcripts detected by reverse transcription PCR (RT-PCR; Fig I.3D,E) and suggests that CDK9 is important for the correct positioning of 3 '-end processing proteins. This is the first report to show that the increase in the read-through and polyadenylation of replication-dependent histone gene transcripts is associated with an increased detection of RNAPII at the downstream polyadenylation site. The non-linear relationship between the increases in the read-through of RNAPII, as detected by ChIP analysis (Fig I.4E), and polyadenylated mRNA levels, as detected by RT-PCR (Fig I.3D,E), are common in transcriptional studies and might be due to factors such as mRNA stability, RNAPII processivity and differences in RNA processing.

Although CDK9 is known to regulate transcription-associated nuclear processes such as splicing and polyadenylation, the significance of CDK9-directed chromatin modifications in these or other nuclear processes have remained unexplored. We have 
shown that $\mathrm{H} 2 \mathrm{Bub} 1$ is a CDK9-dependent histone modification that directs replicationdependent histone mRNA 3'-end processing by the SLBP-U7 snRNP complex. We propose that CDK9 regulates this, at least in part, by directing $\mathrm{H} 2 \mathrm{Bub} 1$, which might influence the accessibility of the newly synthesized mRNA or the recruitment of 3'-end processing machinery by modifying the chromatin structure. Consistent with a role for chromatin structure in histone mRNA 3'-end processing, the histone variants H3.3 and $\mathrm{H} 2 \mathrm{Av}$ and the histone chaperone Anti-silencing function 1 (Asf1) are necessary for correct histone mRNA 3'-end formation in Drosophila (Wagner et al., 2007). However, the presence of these histone variants on the replication-dependent histone genes and their roles in mammals remain unknown. In our model, specific CDK9-dependent phosphorylations and changes in H2Bub1 would act as markers for the correct recognition of the histone mRNA 3'-end cleavage site. The presence of these markers might be regulated by altering CTD phosphorylation or H2Bub1. In the absence of PSer2 or H2Bub1, RNAPII would continue to transcribe until another termination site (that is, a downstream polyadenylation signal) is reached. Thus, we have revealed a new and unexpected role for CDK9, and a unique cross talk between CDK9 and the histone code in the regulation of histone mRNA 3'-end processing.

\section{Speculation}

In support of the hypothesis that $\mathrm{CDK} 9$ and/or $\mathrm{H} 2 \mathrm{Bub} 1$ regulation of histone mRNA processing might have a role in human disease, it is interesting to note that changes in the expression of polyA ${ }^{+}$replication-dependent histone mRNAs have been revealed by microarray analyses in various subtypes of breast cancer (Abba et al., 2005; Zhao et al., 2004) and oropharyngeal squamous-cell carcinoma (Martinez et al., 2007). Although the function of these transcripts is unclear, it is possible that they provide an advantage to rapidly dividing cells by supplying an additional source of histones outside of the S-phase owing to their cell-cycle-independent stability and translation (Kirsh et $a l ., 1989)$. Future experiments will specifically address this hypothesis.

\section{Methods}

\section{Plasmids and siRNAs}

Cdk9 and Rnf40 were cloned into pcDNA3.1-Hygro (Invitrogen, Heidelberg, Germany). The $\alpha$-amanitin-resistant RNAPII constructs were described previously (Chapman et al., 2007). The siRNAs used are listed in Supp Table I.S4. 


\section{Cell culture and transfection}

H1299, HEK293 and U-2 OS cells were grown in high-glucose DMEM containing 10\% fetal bovine serum $(\mathrm{FBS})$ and $1 \times$ penicillin-streptomycin. Cells were treated with KM05283 (Thermo Fisher Scientific International), DRB (Sigma, Munich, Germany), flavopiridol (Sigma), CDK9 inhibitor II (Merck KGaA, Darmstadt, Germany) or nutlin 3 (Sigma) as indicated. Transfections were carried out using Lipofectamine 2000 or Lipofectamine RNAiMAX (Invitrogen) according to the manufacturer's instructions. Studies using the $\alpha$-amanitin-resistant RNAPII constructs were carried out as described previously (Chapman et al., 2007).

\section{Western blot analysis}

Protein samples were analysed by Western blot analysis using the antibodies listed in Supp Table I.S1.

\section{ChIP and gene expression analysis}

ChIP analyses were carried out essentially as described previously (Johnsen et al., 2009; Nelson et al., 2006) using the antibodies and dilutions listed in Supp Table I.S1. RNA was harvested with TRIZOL (Invitrogen) according to the manufacturer's instructions and reverse transcribed using either oligo-dT or random nonamer primers as indicated in the respective figure legends. DNA samples (ChIP or cDNA) were analysed by using real-time PCR using the iQ SYBR Green Supermix (BioRad, Munich, Germany) with the primers listed in Supp Tables I.S2 and I.S3. Experimental background was determined by carrying out a control ChIP with either a non-specific IgG and/or no primary antibody. The averages of the background signals for all primer pairs were similar and are graphically represented by the dotted line (Fig I.1C,D; Fig I.4A-D). The percentage of total transcripts for the processed and read-through HIST1H2BD transcripts shown in Fig I.3C was determined by RT-PCR using random primed cDNA from control or CDK9-siRNA-transfected cells and dividing the signal for the read through' transcript by the total amount of transcript. The remaining fraction of total transcript was interpreted as the processed fraction. All quantitative RT-PCRs were normalized to $36 B 4$ or MTRNR2 before additional normalization and statistical analyses. 


\subsubsection{Supplementary Material}

\section{Supplementary Detailed Materials and Methods}

\section{Chromatin immunoprecipitation}

ChIP analyses were performed essentially as described (Nelson et al., 2006) using the antibodies and dilutions listed in Supp Table I.S1. Briefly, medium was removed from the cell culture plates and DNA binding complexes were cross-linked by adding PBS containing $1.42 \%$ formaldehyde to the cells for $15 \mathrm{~min}$ at room temperature. Cross-linking was quenched by adding $1.25 \mathrm{M}$ glycine to a final concentration of $125 \mathrm{mM}$ and incubated at room temperature for an additional $5 \mathrm{~min}$. Cross-linked cells were then washed twice with ice-cold PBS prior to adding $1 \mathrm{ml}$ IP Buffer (150 mM NaCl, $50 \mathrm{mM}$ Tris-HCl (pH 7.5), $5 \mathrm{mM}$ EDTA, 0.5\% (v/v) NP-40, $1.0 \%$ (v/v) Triton X-100 containing $1 \mathrm{mM}$ NEM, $10 \mathrm{mM}$ BGP and $1 \times$ PIC). Cells were scraped, transferred to $1.5 \mathrm{ml}$ tubes and pelleted by centrifugation $(12,000 \times \mathrm{g})$ at $4{ }^{\circ} \mathrm{C}$. The nuclear pellets were washed an additional time with IP buffer before resuspending in $300 \mu \mathrm{l}$ IP buffer. Samples were sonicated using a Bioruptor (Diagenode, Liège, Belgium) on " $\mathrm{H}$ " setting 3 times for 10 min each with alternating pulses and pauses for $10 \mathrm{~s}$ each. Sonicated chromatin was centrifuged at full speed and pre-cleared with 100 $\mu 1$ of 50\% Sepharose 4B (GE Healthcare, Piscataway, NJ) slurry in IP Buffer for $1 \mathrm{~h}$ at $4{ }^{\circ} \mathrm{C}$. After centrifugation, pre-cleared chromatin was diluted, aliquoted, shock frozen in liquid nitrogen, and stored at $-80^{\circ} \mathrm{C}$. For ChIP analysis, $50 \mu 1$ of chromatin was diluted to a final volume of $500 \mu \mathrm{l}$ in IP buffer containing NEM, BGP and PIC and incubated overnight with the indicated amounts of antibody (see Supp Table I.S1). Chromatin complexes were captured by adding $30 \mu \mathrm{l}$ of a $50 \%$ Protein-A or Protein-G Sepharose slurry (GE Healthcare) and incubated for an additional $2 \mathrm{~h}$ with tumbling at $4{ }^{\circ} \mathrm{C}$. Samples were centrifuged at $2000 \times \mathrm{g}$ for $2 \mathrm{~min}$ at $4{ }^{\circ} \mathrm{C}$ and washed 6 times with IP buffer. The immunoprecipitated chromatin complexes were reverse cross-linked by adding $100 \mu 1$ 10\% (w/v) Chelex 100 (BioRad, Hercules, CA) slurry and heating at 95 ${ }^{\circ} \mathrm{C}$ for $10 \mathrm{~min}$. Two $\mu \mathrm{l}$ proteinase $\mathrm{K}(20 \mu \mathrm{g} / \mu \mathrm{l}$, Invitrogen) was added to each sample and incubated at $55{ }^{\circ} \mathrm{C}$ for $30 \mathrm{~min}$. Proteinase $\mathrm{K}$ was then inactivated by incubating at $95{ }^{\circ} \mathrm{C}$ for $10 \mathrm{~min}$. Finally, samples were centrifuged at $12,000 \times \mathrm{g}$ for $1 \mathrm{~min}$ and the supernatant was transferred to a new tube. For normalization, input samples were prepared by adding $1 \mu \mathrm{l}$ GlycoBlue $(15 \mu \mathrm{g} / \mu \mathrm{l}$, Applied Biosystems, Austin, TX) to 50 $\mu \mathrm{l}$ of chromatin extract and precipitated by adding 2.5-3 volumes of ethanol and 
incubating overnight at $-20{ }^{\circ} \mathrm{C}$. Input samples were then centrifuged at $12,000 \times \mathrm{g}$ and washed twice with $70 \%$ ethanol before preparing DNA by the Chelex method described above.

\section{Gene expression analysis and real-time PCR}

RNA was harvested using the TRIZOL method (Invitrogen) according to the manufacturer's instructions. Reverse transcriptions were performed using either oligo$\mathrm{dT}\left(\mathrm{T}_{23} \mathrm{VN}\right)$ or random nonamer primers as indicated below or in the respective figure legends. One $\mu \mathrm{g}$ total RNA was mixed with $2 \mu \mathrm{l}$ random nonamer $(15 \mu \mathrm{M})$ or oligo-dT $(50 \mu \mathrm{M})$ primers (Metabion, Martinsried, Germany), $4 \mu \mathrm{l}$ dNTPs (2.5 mM, Promega), and brought to a volume of $16 \mu \mathrm{l}$ in RNAse-free water. Samples were heated at $70{ }^{\circ} \mathrm{C}$ for $5 \mathrm{~min}$ and placed on ice. A total volume of $4 \mu \mathrm{l}$ containing $2 \mu 110 \times$ reaction buffer, $0.25 \mu \mathrm{l}$ (10 U) RNAse inhibitor (New England Biolabs, Ipswich, MA), and $0.125 \mu 1$ (25 U) M-MuLV reverse transcriptase (New England Biolabs) was added to each sample and incubated at $42{ }^{\circ} \mathrm{C}$ for $1 \mathrm{~h}$. Finally, the reverse transcriptase was inactivated by heating at $95{ }^{\circ} \mathrm{C}$ for $5 \mathrm{~min}$ and samples were diluted to $50 \mu \mathrm{l}$ with water.

Two $\mu 1$ of each DNA (ChIP or cDNA) sample were used for quantitative realtime PCR analysis in a final volume of $25 \mu 1$. Real-time PCR analysis was performed using the iQ SYBR Green Supermix (BioRad) with the primers listed in Supp Tables I.S2 and I.S3. A two-step protocol $\left(10 \mathrm{~min}\right.$ at $95^{\circ} \mathrm{C}$; followed by 40 cycles of: $95^{\circ} \mathrm{C}$ for $15 \mathrm{~s}$ and $60{ }^{\circ} \mathrm{C}$ for $1 \mathrm{~min}$ ) followed by a melting curve analysis was used for each primer pair and performed on a Chromo4 Real-Time PCR System (BioRad). ChIP and input samples were quantified using a standard curve made from ChIP input DNA. ChIP samples were normalized to their appropriate input samples and expressed as "\% input". Experimental background was determined by performing a control ChIP with either a non-specific IgG (see Supp Table I.S1 for specific information) and/or no primary antibody (i.e., Protein-A Sepharose alone). The average of the background signals for all primer pairs were similar and are graphically represented by the dotted line in Fig I.1C-E, Fig I.4A-E and Supp Fig I.S2D. RT-PCR analysis was quantified using a mixture of cDNA (for p21 gene expression analysis) or a genomic DNA dilution curve (for determining the precise " $\%$ of total transcript" for processed and read-through HIST1H2BD transcripts). The percent of total transcript for the processed and readthrough HIST1H2BD transcripts shown in Fig I.3C were determined by RT-PCR using random primed cDNA from control or CDK9 siRNA transfected cells and dividing the 
signal for the "read-through" transcript by the total amount of transcript (for the "\% total transcript" for the read-through transcript) and the remaining fraction of total transcript was interpreted as the processed fraction. A schematic showing the position of the amplicons investigated is displayed in Fig I.3A. Polyadenylated HIST1H2BD and HIST1H2AC were analysed by oligo-dT primed reverse transcription followed by quantitative real-time PCR analysis using primers that lie in the first exon of the respective gene. All qRT-PCRs were normalized to $36 B 4$ or MTRNR2 prior to additional normalization and statistical analyses.

\section{Supplementary Tables}

Supp Table I.S1: Antibodies used for ChIP and western blot analyses and the respective dilutions.

\begin{tabular}{|c|c|c|c|c|c|}
\hline Target Protein & Clone & Cat. Nr. & ChIP & WB & Source \\
\hline CBP80 & - & - & $1 \mu l$ & - & (Izaurralde et al., 1994) \\
\hline CDK9 & $\mathrm{C}-20$ & sc-484 & $1 \mu \mathrm{g}$ & $1: 100$ & Santa Cruz \\
\hline Histone $\mathrm{H} 2 \mathrm{~B}$ & - & $07-731$ & - & $1: 3,000$ & Upstate \\
\hline Histone H2Bub1 & - & - & $75 \mu 1^{\dagger}$ & $1: 1,000^{\dagger}$ & (Minsky et al., 2008) \\
\hline Hsc70 & B6 & sc-7298 & - & $1: 25,000$ & Santa Cruz \\
\hline IgG (Non-specific) & - & ab46540 & $1 \mu \mathrm{g}$ & - & Abcam \\
\hline Lsm11 & - & - & $2 \mu 1$ & - & (Pillai et al., 2003) \\
\hline NELF-E & KM2479 & - & $1 \mu 1$ & - & (Narita et al., 2007) \\
\hline PAF1 & - & $\mathrm{pAb}-015-050$ & $1 \mu \mathrm{g}$ & - & Diagenode \\
\hline RNAPII (N-Term) & $\mathrm{N}-20$ & sc-899 & $1 \mu \mathrm{g}$ & - & Santa Cruz \\
\hline RNAPII (P-Ser2-CTD) & H5 & MMS-129R & $2 \mu 1$ & - & Covance \\
\hline RNAPII (P-Ser2-CTD) & $3 \mathrm{E} 10$ & - & - & $1: 10^{\dagger}$ & (Chapman et al., 2007) \\
\hline RNAPII (P-Ser5-CTD) & $\mathrm{H} 14$ & MMS-134R & $1 \mu 1$ & $1: 10,000$ & Covance \\
\hline RNF20 & - & NB100-2242 & $1 \mu \mathrm{g}$ & $1: 2,000$ & Novus Biologicals \\
\hline RNF20 & - & ab32629 & - & - & Abcam \\
\hline RNF40 & - & ab26082 & $1 \mu \mathrm{g}$ & $1: 1,000$ & Abcam \\
\hline SLBP & - & - & $2 \mu 1$ & - & (Arnold et al., 2008) \\
\hline TBP & SI-1 & sc-273 & $0.5 \mu \mathrm{g}$ & - & Santa Cruz \\
\hline Goat Anti-Mouse IgM & - & M 8644 & $3 \mu \mathrm{g}$ & - & Sigma \\
\hline $\begin{array}{l}\text { Donkey Anti-Mouse } \\
\text { IgG-HRP }\end{array}$ & - & $715-036-150$ & - & $1: 10,000$ & $\begin{array}{l}\text { Jackson } \\
\text { ImmunoResearch }\end{array}$ \\
\hline $\begin{array}{l}\text { Donkey Anti-Mouse } \\
\text { IgM-HRP }\end{array}$ & - & $115-035-044$ & - & $1: 3,000$ & $\begin{array}{l}\text { Jackson } \\
\text { ImmunoResearch }\end{array}$ \\
\hline $\begin{array}{l}\text { Donkey Anti-Rabbit } \\
\text { IgG-HRP }\end{array}$ & - & $711-036-152$ & - & $1: 10,000$ & $\begin{array}{l}\text { Jackson } \\
\text { ImmunoResearch }\end{array}$ \\
\hline $\begin{array}{l}\text { Goat Anti-Rat IgG + } \\
\text { IgM-HRP }\end{array}$ & - & $112-035-068$ & - & $1: 10,000$ & $\begin{array}{l}\text { Jackson } \\
\text { ImmunoResearch }\end{array}$ \\
\hline
\end{tabular}


Supp Table I.S2: Primers utilized in ChIP in 5' to 3' orientation. Primers not obtained from other previous studies were designed using the Primer3 program (http://frodo.wi.mit.edu/).

\begin{tabular}{lll} 
ChIP Primers & & Source \\
\hline HIST1H2BD TSS F & ACCTCATTTGAATACCGCATCT & This study \\
HIST1H2BD TSS R & TGGTAGGTTCAGGCATCGTAG & This study \\
HIST1H2BD +0.5kb F & TCTTTTAAGAGCCACGCATGT & This study \\
HIST1H2BD +0.5kb R & CGTAACATTAAAGATTGGTACTGTGG & This study \\
HIST1H2BD +13kb F & TGCCATGGACTACCTTTGCT & This study \\
HIST1H2BD +13kb R & AGGTTTGGATCAACAATGAAGG & This study \\
HIST1H2AC TSS F & AAAAGCGGCCATGTTTTACA & This study \\
HIST1H2AC TSS R & AAAAATCACCAAAACCAGCG & This study \\
HIST1H2AC +15kb F & AGATTTCCCAAATTCCAGCC & This study \\
HIST1H2AC +15kb R & AAGGAAGAAAGGAAATGGCAA & This study \\
p21 TSS F & GGGGCGGTTGTATATCAGG & This study \\
p21 TSS R & GGCTCCACAAGGAACTGACTTC & (Gomes et al., 2006) \\
p21 5.3kb F & CCAGGGCCTTCCTTGTATCTCT & (Gomes et al., 2006) \\
p21 5.3kb R & ACATCCCCAGCCGGTTCT & (Gomes et al., 2006)
\end{tabular}

Supp Table I.S3: Primers utilized in RT-PCR in 5' to 3' orientation. Primers not obtained from other previous studies were designed using the Primer3 program (http://frodo.wi.mit.edu/).

\begin{tabular}{lll} 
RT-PCR Primers & & Source \\
\hline 36B4 F & GATTGGCTACCCAACTGTTG & (Fritah et al., 2005) \\
36B4 R & CAGGGGCAGCAGCCACAAA & (Fritah et al., 2005) \\
HIST1H2BD Total F & ACGATGCCTGAACCTACCAA & This study \\
HIST1H2BD Total R & AGCCTTAGTCACCGCCTTCT & This study \\
HIST1H2BD PolyA F & CCAACTCATCCTGGTTTGCT & This study \\
HIST1H2BD PolyA R & TCCCCTCGGTAACCTTCTTT & This study \\
HIST2H2AA Total / PolyA F & AAGGCAAAGGGCAAGTGAG & This study \\
HIST2H2AA Total R & AAGAGCCTTTTGAGTTCACAGGT & This study \\
HIST2H2AA PolyA R & TGAAAACGTGGGTGGCTCT & This study \\
HIST1H2AC Total F & GACGAGGAGCTCAACAAACTG & This study \\
HIST1H2AC Total R & ACCTGTCAAATCACTTGCCC & This study \\
HIST1H2AC PolyA F & CCTGTCCACTGTTGGTAGGC & This study \\
HIST1H2AC PolyA R & TTCACTTACCACCATTCCAGC & This study \\
MTRNR2 F & CATAAGCCTGCGTCAGATCA & This study \\
MTRNR2 R & CCTGTGTTGGGTTGACAGTG & This study \\
p21 F & CCAGCTGGGCTCTGCAATT & (Gomes et al., 2006) \\
p21 R & GCTGAGAGGGTACTGAAGGGAAA & (Gomes et al., 2006)
\end{tabular}


Supp Table I.S4: siRNAs utilized in a 5' to 3' orientation.

\begin{tabular}{|c|c|c|c|}
\hline Target Gene & siRNA Target Sequence & Source & Reference \\
\hline CDK9 (\#1) & UAG GGA CAU GAA GGC UGC UAA & Qiagen & \\
\hline CDK9 (\#2) & GUC AAC UUG AUU GAG AUU UGU CGA A & Invitrogen & \\
\hline CDK9 (\#3) & GCA AGG GUA GUA UAU ACC UGG UGU U & Invitrogen & \\
\hline CTR9 (\#1) & UUC CGU UAC CAC GUC AUA UAA & Qiagen & \\
\hline CTR9 (\#2) & UAG CAA CCC UAU GGU AUU GAA & Qiagen & \\
\hline CyclinT1 & AAT CCC TTC CTG ATA CTA GAA & Qiagen & (Chiu et al., 2004) \\
\hline NELF-E & AAG UCA ACA UAG CCC GAA AUU & Ambion & \\
\hline RNF20 & $\begin{array}{l}\text { SmartPool@ containing: } \\
\text { CCA AUG AAA UCA AGU CUA AUU, } \\
\text { UAA GGA AAC UCC AGA AUA UUU, } \\
\text { GCA AAU GUC CCA AGU GUA AUU, } \\
\text { AGA AGA AGC UAC AUG AUU UUU }\end{array}$ & Dharmacon & \\
\hline RNF20 \#1 & CAG CGA CUC AAC CGA CAC UUA & Ambion & \\
\hline RNF20 \#2 & AGC UCU UAU CCC GGA AGC UAA & Ambion & \\
\hline RNF20 \#3 & CAG GUC CGC AAG GAG UAU GAA & Ambion & \\
\hline RNF40 & $\begin{array}{l}\text { SmartPool® containing: } \\
\text { GAG AUG CGC CAC CUG AUU AUU, } \\
\text { GAU GCC AAC UUU AAG CUA AUU, } \\
\text { GAU CAA GGC CAA CCA GAU UUU, } \\
\text { CAA CGA GUC UCU GCA AGU GUU }\end{array}$ & Dharmacon & \\
\hline RNF40 \#1 & AGA GAU GGA UGU GAC AGG UCA & Ambion & \\
\hline RNF40 \#2 & TGA GGA CAU GCA GGA ACA GAA & Ambion & \\
\hline RNF40 \#3 & AGG UAC UAC AGU UCA AGA ACA & Ambion & \\
\hline SUPT5H & AAC UGG GCG AGU AUU ACA UGA & Qiagen & (Ping et al., 2004) \\
\hline
\end{tabular}


Supplementary Figures

A

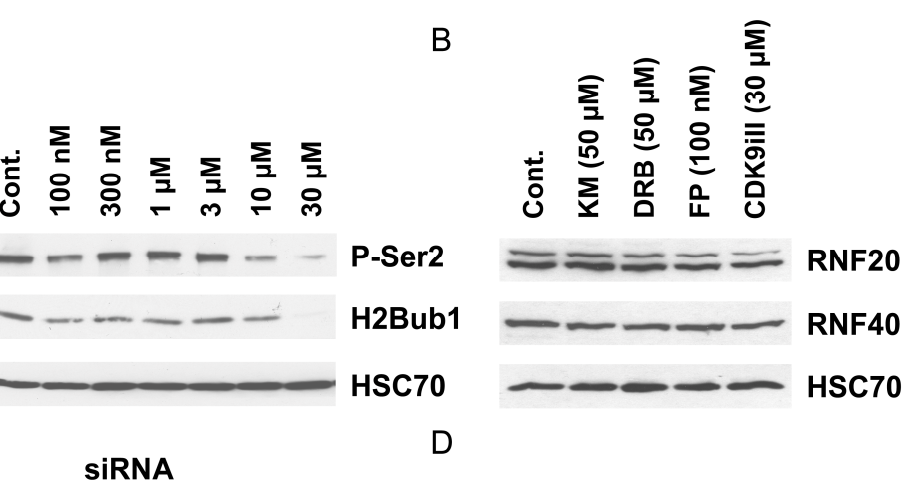

C
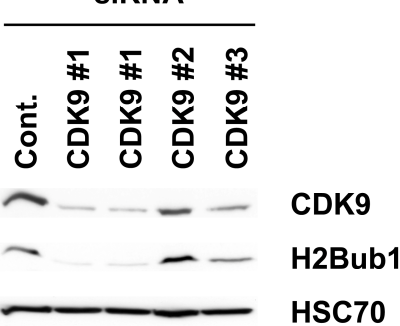

$\mathrm{E}$

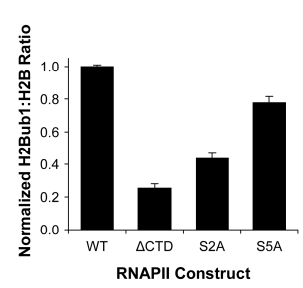

G

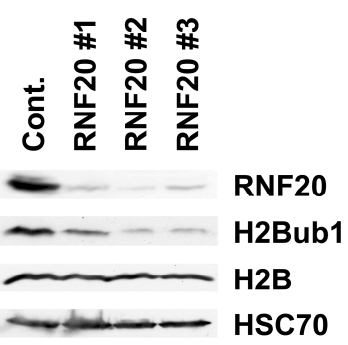

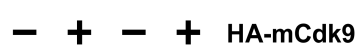

$-\quad++$ CDK9 siRnA
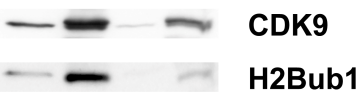

$\mathrm{F}$

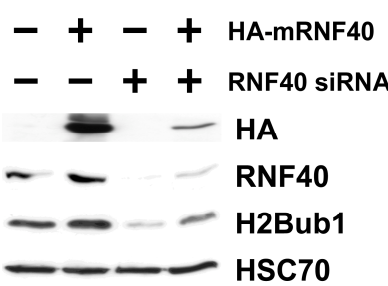

$\mathrm{H}$

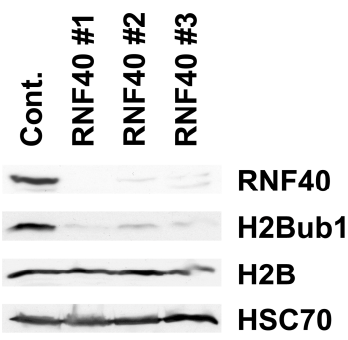

- - HSC70

Supp Fig I.S1: CDK9, RNF20 and RNF40 are essential for maintaining global levels of H2B monoubiquitination. (A) The CDK9 inhibitor II (CDK9iII) dose-dependently decreases P-Ser2-RNAPIICTD and H2Bub1 levels with similar effective concentrations. Cells were treated with different concentrations of CDK9iII and analysed by Western blot for the presence of P-Ser2, H2Bub1 and HSC70 (loading control). (B) RNF20 and -40 levels are unaffected by CDK9 inhibition. The same extracts from H1299 cells treated with CDK9 inhibitors in Fig I.2A were analysed by Western blot for the expression of RNF20, RNFr0 and HSC70. Note that RNF20 and RNF40 expression remain unaltered after $4 \mathrm{~h}$ treatment. (C,D) The effects of CDK9 knock-down are specific. H1299 cells were transfected as in Fig I. 2B with three individual siRNAs against hCDK9. (C) Both siRNAs which efficiently targeted CDK9 $(\# 1,3)$ also efficiently decreased H2Bub1 levels. (D) Over-expression of mCdk9 rescues the H2Bub1 phenotype caused by hCDK9 knock-down. H1299 cells were co-transfected with different combinations of mCdk9 or control expression vector together with a control or hCDK9 specific siRNA as indicated. HSC70 is shown as a loading control. (E) Densitometric analysis of Western blot data from Fig I.2D. The intensity of the specific H2Bub1 signal was normalized to its respective $\mathrm{H} 2 \mathrm{~B}$ control and displayed as "Normalized H2Bub1:H2B Ratio"; mean values, error bars show the range of the two values. (F-H) RNF20 and RNF40 are essential for H2B monoubiquitination. (F) siRNA knockdown and rescue was performed as in (D) by transfecting different combinations of specific siRNAs against human RNF40 (or control siRNA) together with an HA-tagged mRnf40 or control expression plasmid. Three distinct siRNAs targeting RNF20 (G) or RNF40 (H) each result in dramatically decreased levels of H2Bub1 and the respective targeted protein. HSC70 is shown as a loading control. 
A

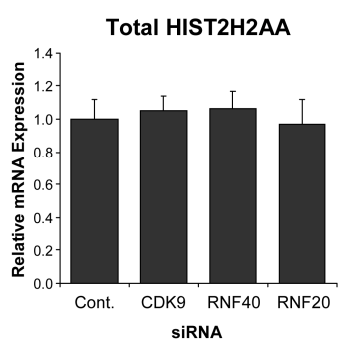

B

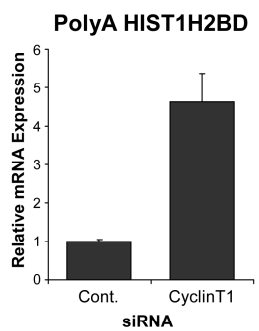

C

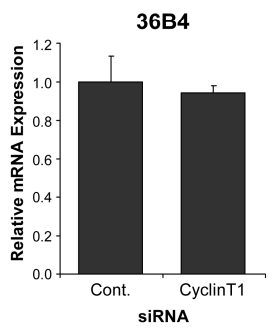

D

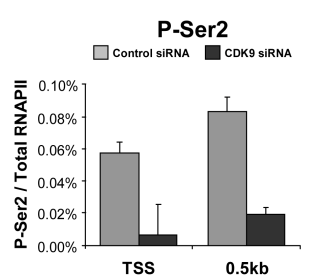

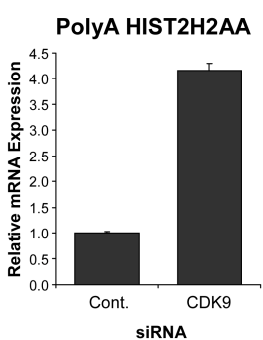
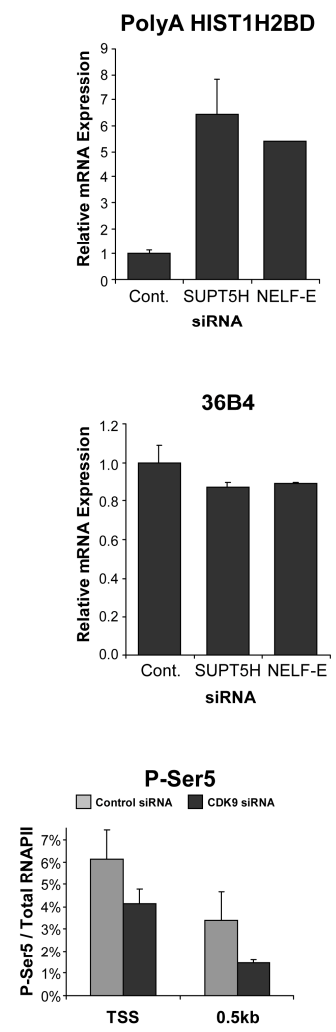

PolyA HIST2H2AA
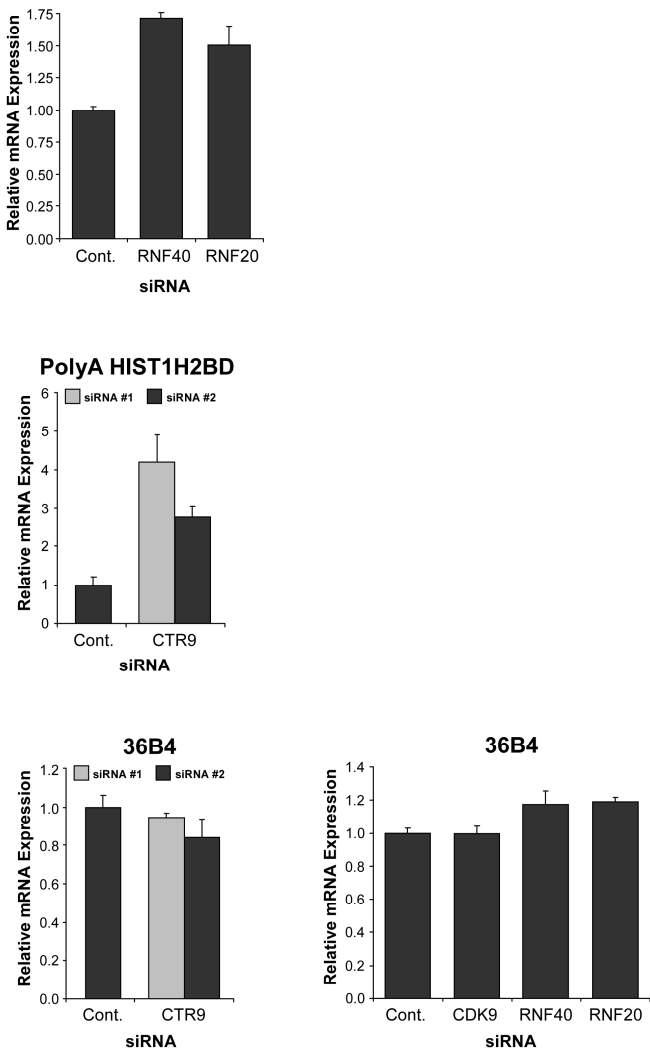

E

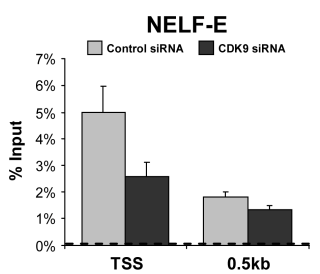

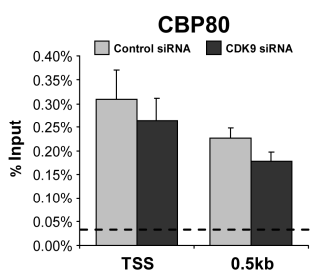

Supp Fig I.S2: CDK9, RNF20/40 and associated proteins and chromatin modifying enzymes specifically affect replication-dependent histone mRNA polyadenylation. (A) The cDNA samples analysed in Fig I.3 were additionally analysed for the expression of total and polyadenylated HIST2H2AA. (B) The knockdown of CyclinT1, SUPT5H, NELF-E, or CTR9 all increase HIST1H2BD polyadenylation. cDNA from $\mathrm{H} 1299$ cells that were transfected as in Fig I.3 with the indicated siRNAs were analysed for total and polyadenylated HIST1H2BD mRNA. The ratio of polyadenylated to total mRNA was normalized to the control and expressed as "relative mRNA expression"; mean values + s.d., $n=3$. (C) PolyT reverse transcribed cDNA samples from B and Fig I.3 were analysed for the expression of polyadenylated 36B4 mRNA. In order to avoid any non-specific effects due to general effects, samples were not normalized to an internal control. Values are graphed relative to the control sample and are expressed as "relative mRNA expression"; mean values + s.d., $n=3$. (D) Data for P-Ser2 and P-Ser5 shown in Fig I.4B was subtracted from the background values and normalized to the amount of total RNAPII. The data is expressed as relative \% of total RNAPII ("P-Ser2 or 5 / Total RNAPII"); mean values + s.d., $n=3$. (E) CDK9 knockdown affects NELF-E recruitment in a similar manner as RNAPII and has no effect on CBP80 recruitment to the HISTIH2BD gene. ChIP extracts analysed in Fig I.4 were examined for the effects on NELF-E and CBP80 recruitment to the HISTIH2BD gene. Note that NELF-E recruitment essentially follows RNAPII (see Fig I.4B), while CBP80 is unaffected. ChIP experiments are displayed as "\% input" as in Fig I.1 and I.4; mean values + s.d., $n=3$. 


\subsection{Publication II}

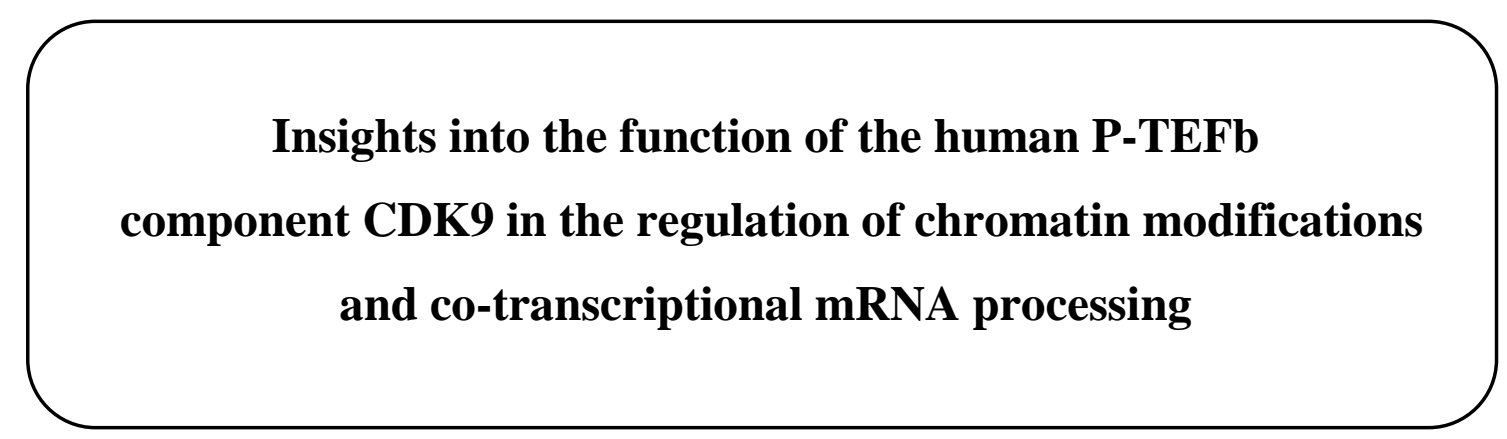

\section{Citation}

Judith Pirngruber, Andrei Shchebet and Steven A. Johnsen

Insights into the function of the human $\mathrm{P}-\mathrm{TEFb}$ component $\mathrm{CDK} 9$ in the regulation of chromatin modifications and co-transcriptional mRNA processing

Cell Cycle (2009), 8(22): 3636-3642

\section{Own contribution}

Preparation and accomplishment of all biological experiments, i.e. transfection of described siRNAs, western blot analysis, gene expression analysis, real-time PCR; complete figure layout, tables, supplemental materials and methods; contribution to the writing of the manuscript (parts of introduction, methods and results section). 


\begin{abstract}
Cyclin-dependent kinase-9 (CDK9) was originally characterized as a transcription elongation factor which regulates RNA Polymerase II (RNAPII) activity following transcriptional initiation. However, recent evidence from a number of studies have shown that CDK9 plays an important role in regulating not only RNAPII activity but also co-transcriptional histone modification and mRNA processing events such as splicing and 3' end processing. Importantly, our previous work and the work presented here demonstrate that CDK9 functions to guide a complex network of chromatin modifications including histone $\mathrm{H} 2 \mathrm{~B}$ monoubiquitination (H2Bub1), H3 lysine 4 trimethylation (H3K4me3) and H3K36me3. This function appears to be dependent upon not only the phosphorylation of the RNA Polymerase II C-terminal domain but also upon other CDK9 targets such as the Suppressor of Ty Homolog-5 (SUPT5H), Negative Elongation Factor-E (NELF-E) and probably the human Rad6 homolog UBE2A. We provide a working model by which CDK9 may control co-transcriptional replicationdependent histone mRNA 3' end processing in an H2Bub1 and H3K4me3-dependent manner and uncover new and important differences between the functions of human CDK9 and its yeast counterparts Ctk1 and Bur1.
\end{abstract}

\title{
Introduction
}

The active form of the human Positive Transcription Elongation Factor-b (P$\mathrm{TEFb}$ ) complex is composed of its kinase component Cyclin-Dependent Kinase-9 (CDK9) and a cyclin subunit which is most frequently CyclinT1, but may also be CyclinT2A, T2B or K (Romano and Giordano, 2008). The function of P-TEFb in humans has primarily been attributed to the phosphorylation of its substrate proteins which include Ser2 of the heptapeptide C-terminal repeat domain (CTD) of the RNA Polymerase II (RNAPII), the Suppressor of Ty Homolog-5 (SUPT5H) and Negative Elongation Factor-E (NELF-E). Phosphorylation of the RNAPII-CTD plays an important role in transcriptional regulation and co-transcriptional mRNA processing in vivo by serving as a binding platform for factors involved in mRNA 5' end capping, splicing, and 3' end processing as well as chromatin modifications (Buratowski, 2003; Egloff and Murphy, 2008). Phosphorylation of NELF-E removes a block to early transcriptional elongation induced by binding of the NELF complex to the nascent transcript (Yamaguchi et al., 1999; Yamaguchi et al., 2002). Interestingly, phosphorylation of a C-terminal repeat on the SUPT5H protein, which is reminiscent of 
the RNAPII-CTD, appears to convert it from a negative to a positive elongation factor (Yamada et al., 2006). Although additional interesting CDK9 targets such as MyoD (Simone et al., 2002b), PPAR $\gamma$ (Iankova et al., 2006), p53 (Claudio et al., 2006; Radhakrishnan and Gartel, 2006) and Rb (De Luca et al., 1997; Grana et al., 1994; Simone et al., 2002a) have been reported, the RNAPII-CTD, NELF-E and SUPT5H appear to be the major targets of CDK9 which are involved in general transcriptional and co-transcriptional processes in most cell types.

\section{Regulation of Histone Modifications by CDK9}

One of the important functions of RNAPII-CTD phosphorylation is the recruitment of chromatin modifying enzymes. In particular, both Ser5 phosphorylation (by the CDK7-containing TFIIH complex) and Ser2 phosphorylation affect the binding of chromatin modifying enzymes such as the histone $\mathrm{H} 3$ lysine 36 (H3K36) methylating enzyme Set2 in yeast and SETD2 in humans (Egloff and Murphy, 2008). Although several chromatin modifications have been identified and linked to processes such as transcription and DNA replication, the regulation of these modifications and their roles in additional nuclear processes such as RNA processing are relatively unknown. In our previous work, we showed that the inhibition or knockdown of CDK9 resulted in a nearly complete loss of H2Bub1 (Pirngruber et al., 2009b). Since H3K4me3 and H3K79me3 were shown to be dependent upon H2Bub1 in humans (Kim et al., 2005a; Zhu et al., 2005b) and since the binding of SETD2 and subsequent H3K36me3 depends upon RNAPII-CTD-Ser2 phosphorylation, we also tested whether CDK9 knockdown also influences H3K4me3 and H3K36me3. Indeed, not only histone H2B monoubiquitination (Pirngruber et al., 2009b) but also H3K4me3 (Fig II.1A) is a CDK9-dependent histone modifications. We also confirmed for the first time in humans that CDK9-mediated Ser2-RNAPII-CTD phosphorylation is also necessary for maintaining global H3K36me3 levels (Fig II.1A) as has been described in yeast (Kizer et al., 2005; Li et al., 2005a). These results are supported by an earlier study where the presence of both $\mathrm{H} 3 \mathrm{~K} 4 \mathrm{me} 3$ and H3K36me3 on the HIV1 gene was shown to decrease following CDK9 inhibition by flavopiridol (Zhou et al., 2004). 
A

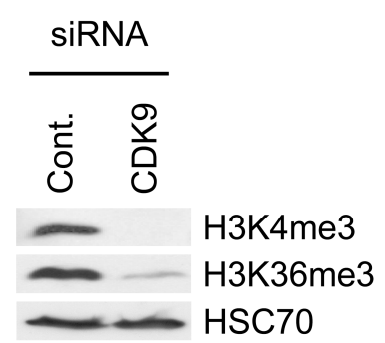

B

SiRNA

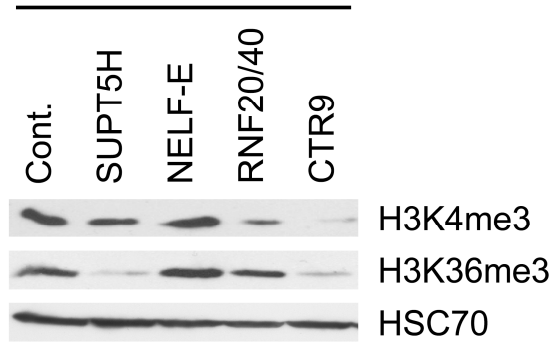

C

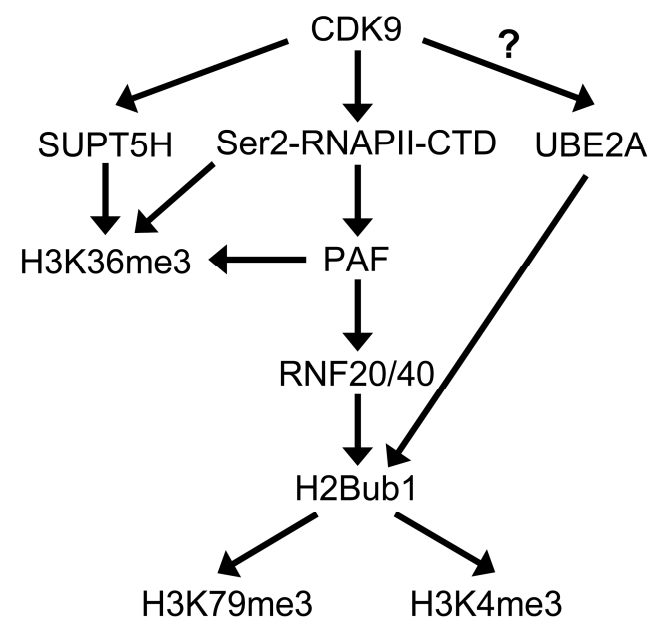

Fig II.1: CDK9 activity is essential for H3K4me3 and H3K36me3. (A and B) H1299 cells were transfected with control or CDK9 siRNAs (A) or siRNAs against SUPT5H, NELF-E, RNF20/40 and CTR9 (B). Histone modifications were analyzed by western blot using the indicated siRNAs and antibodies. HSC70 is shown as a loading control. (C) CDK9 regulates a hierarchy of histone modifications by CTD-dependent and independent mechanisms. Phosphorylation of Ser2 of the RNAPII CTD increases the recruitment of the PAF complex thereby increasing H3K36me3, H2Bub1 and the downstream modifications H3K4me3 and H3K79me3. Additionally, CDK9-dependent phosphorylation of SUPT5H and UBE2A may be necessary for maintaining H3K36me3 and H2Bub1, respectively. 


\section{The Role of Other CDK9 Substrates in Controlling Histone Modifications}

We have shown that CDK9-dependent phosphorylation at Ser2, rather than Ser5 or Ser7 of the RNAPII C-terminal domain, and not transcription per se, is necessary for H2B monoubiquitination (Pirngruber et al., 2009b). However, while these effects were dramatic, they were not complete and some H2Bub1 remained in the absence of P-Ser2RNAPII-CTD. Since SUPT5H and NELF-E are also phosphorylated by CDK9 we performed knockdown analyses of each of these proteins and examined their effects on H2Bub1, H3K4me3 and H3K36me3. Surprisingly, SUPT5H knockdown significantly decreased H3K36me3 (Fig II.1B) levels and moderately affected H2Bub1 (Pirngruber et al., 2009b) and H3K4me3 (Fig II.1B). In contrast, NELF-E knockdown did not appreciably affect any of the modifications. Thus CDK9 appears to regulate H2Bub1 primarily in a CTD-dependent manner through phosphorylation of Ser2 but may also utilize additional CTD-independent ways such as by phosphorylation of SUPT5H for the regulation of $\mathrm{H} 3 \mathrm{~K} 36 \mathrm{me} 3$.

Based on the established role of the PAF complex in regulating H2Bub1 together with RNF20/40 we verified its effects on H2Bub1, H3K4me3 and H3K36me3 by knocking down the PAF component CTR9. As previously reported (Zhu et al., 2005b), CTR9 knockdown decreased global H2Bub1 (Pirngruber et al., 2009b) and H3K4me3 levels (Fig II.1B). Also consistent with data from yeast (Chu et al., 2007), CTR9 knockdown additionally resulted in a decrease in H3K36me3 (Fig II.1B). Thus these three modifications show a similar profile of inhibition following knockdown of CDK9 or the PAF complex. The role of the PAF complex downstream of CDK9 was also supported by our ChIP studies in which PAF recruitment decreased following CDK9 knockdown (Pirngruber et al., 2009b). Also, consistent with previous reports (Kim et al., 2005a; Zhu et al., 2005b), both H2Bub1 and H3K4me3, but not H3K36me3 decreased following knockdown of RNF20 or 40 (Fig II.1B and ref. (Pirngruber et al., 2009b)).

Thus, in humans CDK9 regulates multiple chromatin modifications including H3K36me3, H3K4me3, H2Bub1 and probably also the H2Bub1-dependent H3K79me3 through a complicated network of phosphorylations and protein interactions. As indicated by our data and shown in Fig II.1C, CDK9 functions to regulate H3K36me3 through both SUPT5H and RNAPII-CTD phosphorylation. In contrast, H2Bub1 is regulated primarily through CDK9-dependent CTD phosphorylation which increases PAF and RNF20/40 recruitment. Similar to the yeast Rad6 protein whose H2B 
ubiquitination activity is controlled by Bur1-mediated phosphorylation of Ser120 (Wood et al., 2005), the activity of the human Rad6 homolog (UBE2A) is also regulated by phosphorylation of Ser120 (Sarcevic et al., 2002). It remains unclear whether UBE2A is also a target of CDK9. However, given the incomplete effects of Ser2RNAPII-CTD mutation on global H2Bub1 levels as compared with CDK9 knockdown or inhibition (Pirngruber et al., 2009b), it is likely that additional CDK9 targets such as UBE2A probably also influence $\mathrm{H} 2 \mathrm{~B}$ monoubiquitination. Further support for the hypothesis that UBE2A is also a CDK9 target is the high degree of homology between yeast Rad6 and UBE2A (69\% identity and 85\% similarity at the amino acid level) as well as the recent discovery that UBE2A controls $\mathrm{H} 2 \mathrm{~B}$ monoubiquitination and H3K4me3 (Kim et al., 2009). However, additional studies will be necessary to address whether UBE2A is indeed a CDK9 target in humans.

\section{CDK9 Controls Replication-Dependent Histone mRNA Processing}

In contrast to most RNAPII synthesized mRNAs, replication-dependent histone mRNAs are not polyadenylated, but instead end in a conserved stemloop structure which is recognized and processed by the stemloop binding protein (SLBP)/U7 snRNP complex (Marzluff et al., 2008). This results in the production of unpolyadenylated mRNAs with a defined 3' end. Interestingly, previous reports have described the production of polyadenylated mRNAs from replication-dependent histone genes in vertebrate cells under certain conditions (Collart et al., 1991; Kirsh et al., 1989; Narita et al., 2007). Furthermore, microarray studies have uncovered a potential connection between p53 activity and replication-dependent histone mRNA polyadenylation (Martinez et al., 2007; Yan et al., 2007). Although the physiological relevance of polyadenylated mRNAs produced from replication-dependent histone genes is not clear, the increased stability and cell cycle-independent translation of these transcripts may provide a certain advantage to both non-dividing normal cells or rapidly dividing tumor cells. Thus, changes in the mode of replication-dependent histone mRNA 3' end processing may represent an important regulatory step in tumorigenesis and normal cellular physiology.

In our recent work we uncovered a novel role for CDK9 in histone mRNA processing and demonstrated that not only CDK9, but also the enzyme complexes that catalyze the CDK9-dependent histone modifications, are necessary for suppressing replication-dependent histone mRNA polyadenylation (Pirngruber et al., 2009b). Our 
newest results demonstrate that the increase in the production of the polyadenylated transcripts following CDK9 knockdown was not due to defects in splicing or spurious intragenic transcriptional initiation since increases in a non-spliced histone transcript (HIST2H2AA) (Pirngruber et al., 2009b) were observed and since similar increases in spliced HIST1H2BD and HIST1H2AC transcripts were also observed using oligonucleotides that span the exon/exon junctions of each spliced transcript which is produced from a long primary transcript (Fig II.2A,B). Similar to the effects observed following the knockdown of RNF20 or 40, knockdown of either ASH2L or SETD2, essential components of the $\mathrm{H} 3 \mathrm{~K} 4$ and $\mathrm{H} 3 \mathrm{~K} 36$ lysine methyl transferase complexes (Edmunds et al., 2008; Steward et al., 2006), also resulted in increases in replicationdependent histone mRNA polyadenylation (Fig II.2C).

In order to test whether the increases in replication-dependent histone mRNA polyadenylation observed following CDK9 or RNF40 knockdown were due to a broader effect on global mRNA polyadenylation we also examined the polyadenylation of a control gene (36B4). None of the siRNAs tested had a significant effect on 36B4 mRNA polyadenylation (Fig II.2D and reviewed in ref. (Pirngruber et al., 2009b)). Given the importance of alternative mRNA cleavage and polyadenylation in controlling gene expression and oncogenic transformation (Mayr and Bartel, 2009) we also tested whether CDK9 or RNF40 knockdown affects alternative mRNA polyadenylation of the dihydrofolate reductase (DHFR) and Cyclin D1 (CCND1) genes which are known to exist in at least two different forms produced from alternative polyadenylation sites (Mayr and Bartel, 2009; Edwalds-Gilbert et al., 1997). Our results indicate that the selection of downstream polyadenylation sites was not increased following CDK9 or RNF40 knockdown (Fig II.2E,F). Thus, the increase in the polyadenylation of replication-dependent histone mRNAs is likely to be specific for this class of mRNAs and not a general defect in polyadenylation site selection. 
A
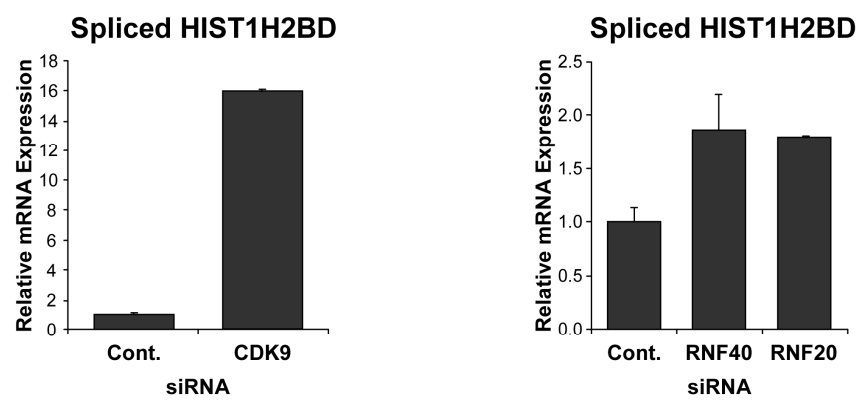

B

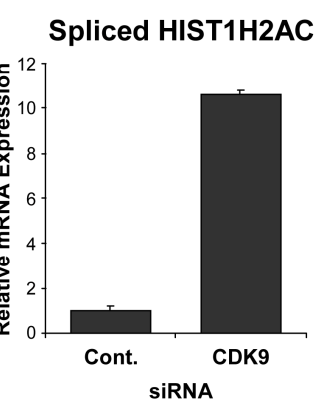

C
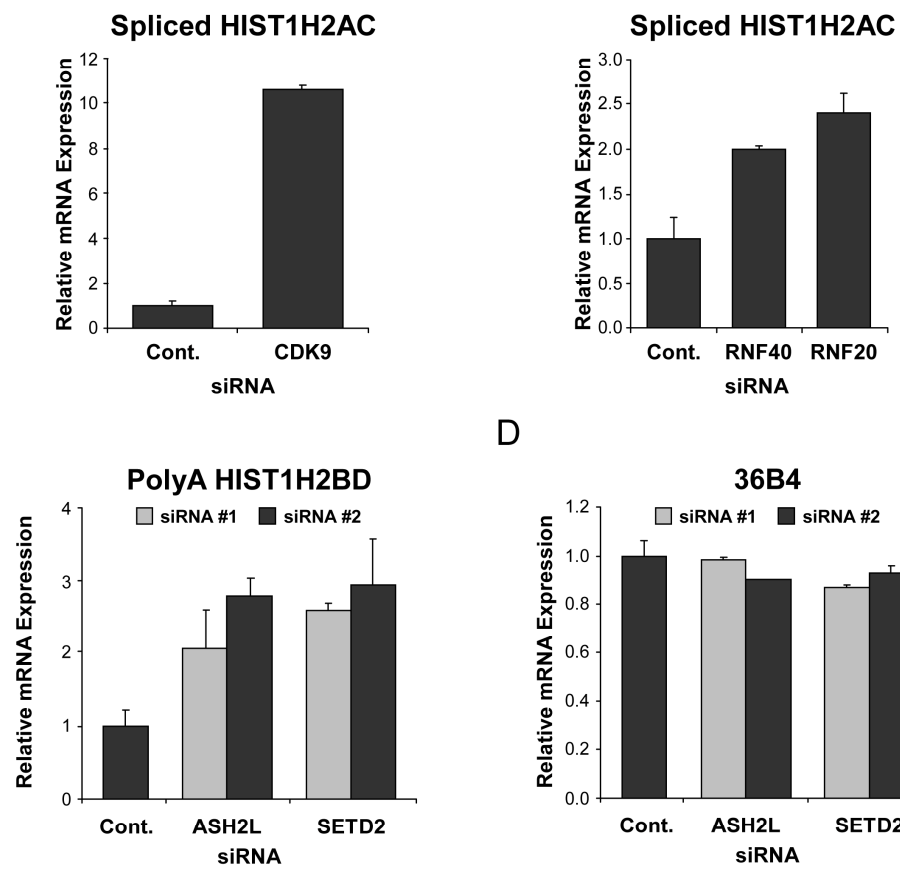

D

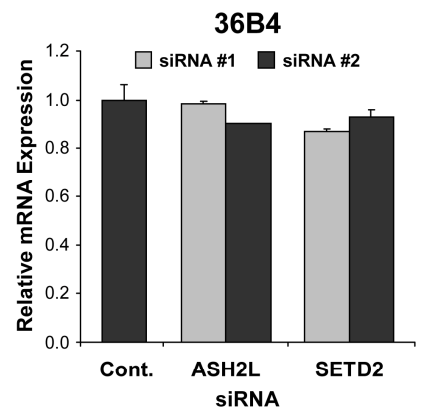

E
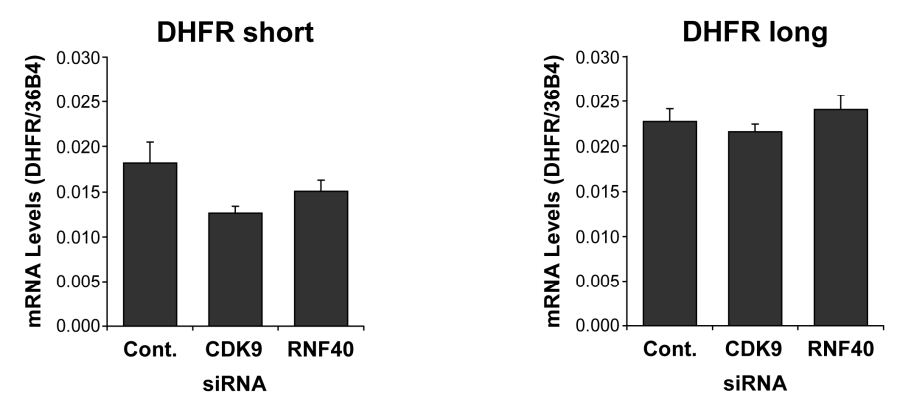

F
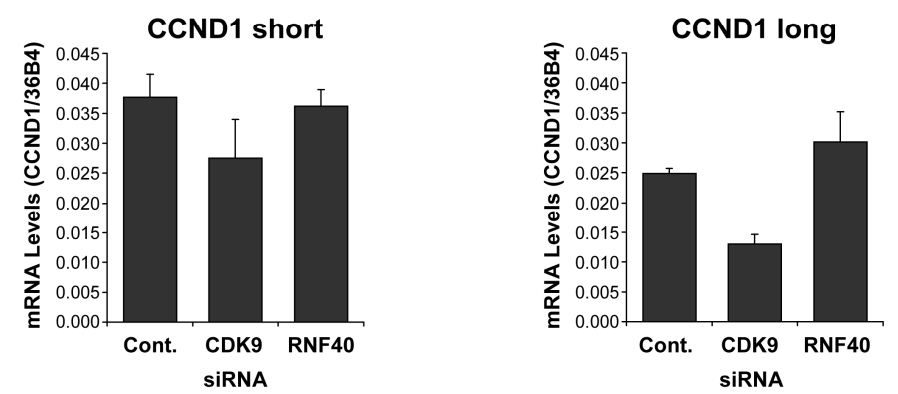
Fig II.2: CDK9 and RNF20/40 knockdown increase the formation of spliced HIST1H2BD (A) and HIST1H2AC (B) transcripts. Total mRNA from cells transfected with control, CDK9, RNF40 or RNF20 siRNA was analyzed by oligo-dT-primed qRT-PCR. Expression levels were normalized to 36B4 gene and expressed as "relative mRNA expression"; mean values + s.d., $n=2$. (C) The knockdown of chromatin modifying enzymes (ASH2L or SETD2) which catalyze modifications downstream of CDK9 increase HIST1H2BD polyadenylation. cDNA from H1299 cells that were transfected with two siRNAs each for ASH2L and SETD2 were analyzed for polyadenylated HIST1H2BD mRNA as previously (Pirngruber et al., 2009b). Expression levels were normalized to $36 B 4$ gene and expressed as "relative mRNA expression"; mean values + s.d., $n=2$. (D) PolyT reverse transcribed cDNA samples from (C) were analyzed for the expression of polyadenylated 36B4 mRNA. In order to avoid any non-specific effects due to general effects, samples were not normalized to an internal control. Values are graphed relative to the control sample and are expressed as "relative mRNA expression"; mean values + s.d., $n=$ 2. (E and F) CDK9 and H2Bub1 do not affect alternative polyadenylation site usage. Both genes, DHFR (E) and CCND1 (F), known to utilize two proximal and distal polyadenylation sites were analyzed for the expression of each transcript. In order to show the relative expression of the different forms to one another, gene expression was only normalized relative to the control gene (36B4) and is shown as "mRNA levels"; mean values + s.d., $n=2$.

\section{Similarities and Distinctions Between P-TEFb Function in Yeast and Humans}

Together these results have uncovered an unexpected connection between RNAPII phosphorylation and covalent histone modifications in controlling a cotranscriptional process distinct from RNAPII activity. Importantly, these results revealed similarities and differences in the functional roles of CDK9 and RNAPII CTD phosphorylation in yeast and humans. The existence of two yeast orthologs of CDK9, which serve at least partially overlapping roles with their human counterpart (Wood et al., 2007), combined with the common functions of these proteins in transcriptional elongation, has led to the general assumption that studies on the yeast Ctk1 and/or Bur1 mutants are directly applicable to the human system. However, as previously discussed by Wood and Shilatifard, there are both important similarities and distinctions between the yeast and human P-TEFb complexes (Wood and Shilatifard, 2006). Our results expand upon the many studies in yeast and demonstrate important distinctions between yeast and mammals. We have shown that CDK9 not only regulates H3K36me3, which is a characteristic of the yeast CTD kinase Ctk1, but also controls H2Bub1 and H3K4me3 which is a function of Bur1 in yeast (Laribee et al., 2005; Wood et al., 2005). A simplistic comparison of the roles of CDK9 with Ctk1 and Bur1 is further complicated by the fact that mutation of Ctk1 leads to an overall increase in H3K4me3 and concomitant decrease in $\mathrm{H} 3 \mathrm{~K} 4 \mathrm{me} 1$ due to increased 3' spreading of $\mathrm{H} 3 \mathrm{~K} 4 \mathrm{me} 3$ into active genes (Xiao et al., 2007). We have also shown that CDK9 regulates PAF recruitment and $\mathrm{H} 2 \mathrm{Bub} 1$, while the opposite seems to be true in yeast where mutation of PAF complex components results in a decrease of P-Ser2 (Mueller et al., 2004) and H2Bub1 acts as a barrier to block Ctk1 recruitment and Ser2 phosphorylation (Wyce et 
al., 2007). Another difference is that while H2Bub1 is dependent upon Bur1 in yeast, this effect appears to be independent of CTD phosphorylation since P-Ser2 is unaffected by the Bur1 mutation (Keogh et al., 2003). Instead, Bur1 phosphorylates and activates the E2 ubiquitin conjugating enzyme Rad6 which is essential for H2Bub1 in yeast (Wood et al., 2005). Since the activity of the human orthologue of Rad6 (UBE2A) is essential for H2Bub1 (Kim et al., 2009) and regulated by phosphorylation (Sarcevic et al., 2002) a possible role of CDK9 in regulating their activity should be further investigated.

Another difference between the functions of human CDK9 and its yeast orthologues may lie in its CTD-independent functions. For example, while H3K36me3 is independent of Spt5 in yeast (Chu et al., 2006) the knockdown of human SUPT5H dramatically decreased H3K36me3 to a similar extent as CDK9. This opens the possibility that H3K36me3 is regulated not only by the interaction of SETD2 with the phosphorylated CTD, but also through CDK9-mediated phosphorylation of SUPT5H. The regulation of NELF is another defining feature of the mammalian system which is not present in yeast. While CDK9 and NELF are generally considered to play opposing roles in transcription, it would seem at first glance that they cooperate in the regulation of replication-dependent histone mRNA processing (Narita et al., 2007; Pirngruber et al., 2009b). However, it is conceivable that these two proteins play distinct roles during different cell cycle phases. For example, in one scenario NELF may serve to repress transcription of the replication-dependent histone genes outside of S-phase whereas CDK9 may be necessary for proper processing during replication. In this way, either derepression of the histone genes in the absence of active SLBP or the deregulation of proper processing during S-phase would similarly give rise to increases in polyadenylated histone transcripts in asynchronous cell cultures. However, to definitively establish the cooperative or opposing roles of these proteins, additional studies using cell cycle synchronized cultures will be necessary. Nevertheless, based on our data, we suggest that CDK9 regulates H2Bub1 largely through phosphorylation of Ser2 of the RNAPII CTD but possibly also through phosphorylation of UBE2A. Our results further suggest that although there are many similarities between the activities of CDK9 in mammals and the actions of Bur1 and Ctk1 in yeast, there are also distinct differences between their functions. 


\section{Regulation of Co-transcriptional mRNA Processing}

The presence of the U7 snRNP and the unique mode of histone mRNA 3' end processing is another defining feature of higher eukaryotic systems. In contrast to yeast, where replication-dependent histone mRNAs are polyadenylated (Osley, 1991), the histone mRNAs of higher eukaryotic organisms contain a conserved stem-loop structure that guides 3' end cleavage by the U7 snRNP (Dominski and Marzluff, 2007). We have shown that CDK9 and its associated downstream histone modifications function to maintain proper 3' end processing of replication-dependent histone mRNAs. Although CDK9 has been connected with transcription-associated nuclear processes such as splicing and polyadenylation, the functional significance of CDK9-directed chromatin modifications in these or other nuclear processes have remained unexplored. We have demonstrated that CDK9 activity helps determine whether replication-dependent histone mRNAs are recognized by the SLBP/U7 snRNP complex or are polyadenylated. As demonstrated by a more modest effect of knocking down RNF20/40 or any of the other individual chromatin modifying enzymes investigated, the regulation of this processing appears to be complex and involves multiple modifications including H2Bub1, H3K4me3 and H3K36me3, and probably also the phosphorylation of SUPT5H and UBE2A.

Based on our results we propose that CDK9 plays a role in determining whether replication-dependent histone mRNAs are cleaved or polyadenylated by directing multiple chromatin modifications including $\mathrm{H} 2 \mathrm{~B}$ monoubiquitination. We hypothesize that these specific CDK9-dependent changes to the CTD and histone codes serve as landmarks for the recruitment of mRNA processing factors and the correct recognition of the histone mRNA 3' end cleavage site. In our model (Fig II.3), CDK9-dependent phosphorylation of Ser2 of the RNAPII CTD serves as a platform for the recruitment of PAF which stimulates the activity of the RNF20/40 complex thereby increasing H2Bub1 levels specifically near the 3' end cleavage site (Fig II.3A). The presence of $\mathrm{H} 3 \mathrm{~K} 4 \mathrm{me} 3$ is not only dependent upon H2Bub1, but as determined by genome-wide ChIP-on-chip and ChIP-seq (Barski et al., 2007; Guenther et al., 2007) it is strategically positioned a few hundred bp 3' to the TSS and can thus serve to recruit the histone mRNA 3' end processing complex in an H2Bub1-dependent manner. Indeed, in this scenario $\mathrm{H} 3 \mathrm{~K} 4 \mathrm{me} 3$ could then affect 3 ' end formation by serving as a binding site for the chromodomain protein CHD1 which subsequently recruits the U2 and U7 snRNP complexes. This is consistent with the identification of CHD1 as a H3K4me3 binding 
protein and both $\mathrm{H} 3 \mathrm{~K} 4 \mathrm{me} 3$ and CHD1 as being essential for the recruitment of the U2 snRNP to chromatin (Sims III et al., 2007). Furthermore, the U2 snRNP was separately shown to stimulate replication-dependent histone mRNA 3' end formation together with the U7 snRNP (Friend et al., 2007). Interestingly, CHD1 was also recently shown to be necessary for the deposition of the variant histone H3.3 (Konev et al., 2007) which is essential for proper histone mRNA 3' end cleavage in Drosophila (Wagner et al., 2007). Similarly, H2Bub1 is vital for nucleosome reassembly in yeast (Fleming et al., 2008) and may therefore also affect variant histone deposition. It is likely that H2Bub1 may function independent of $\mathrm{H} 3 \mathrm{~K} 4 \mathrm{me} 3$ and serve to recruit specific $\underline{\text { Ubiquitin }}$ Interaction Motif-(UIM)-containing proteins such as RAP80 which recognizes H2Bub1 (Wu et al., 2009). In the absence of these landmarks, RNAPII would continue to transcribe until an alternative termination site (i.e., downstream polyadenylation signal) is reached (Fig II.3B). Thus we have revealed a new and unexpected role for CDK9 and a unique cross-talk between CDK9 and the histone code in the regulation of histone mRNA 3' end processing.

Additional studies will be necessary to determine the role of these CDK9dependent histone modifications in other nuclear processes such as DNA replication and splicing. Interestingly, a number of recent studies have uncovered an enrichment of several histone modifications including H3K36me3 specifically on the exonic regions of active genes (Andersson et al., 2009; Kolasinska-Zwierz et al., 2009; Schwartz et al., 2009) providing further support that the regulation of chromatin modifications may play important roles in eliciting the effects of CDK9 activity in co-transcriptional processes. Additional studies will be necessary to examine whether H2Bub1 is also similarly enriched in exonic regions. Although changes in histone mRNA processing may be a common occurrence in human diseases as indicated by differences in polyadenylated replication-dependent histone mRNA expression in several microarray studies (Abba et al., 2005; Ehlers et al., 2008; Martinez et al., 2007; Zhao et al., 2004), the role and regulation of these transcripts remains unknown. The significance of polyadenylated replication-dependent histones must also be determined. Future studies in ours and other laboratories will aim to address each of these points and gain more insight into the significance of CDK9 and chromatin modifications in the regulation of nuclear processes. 

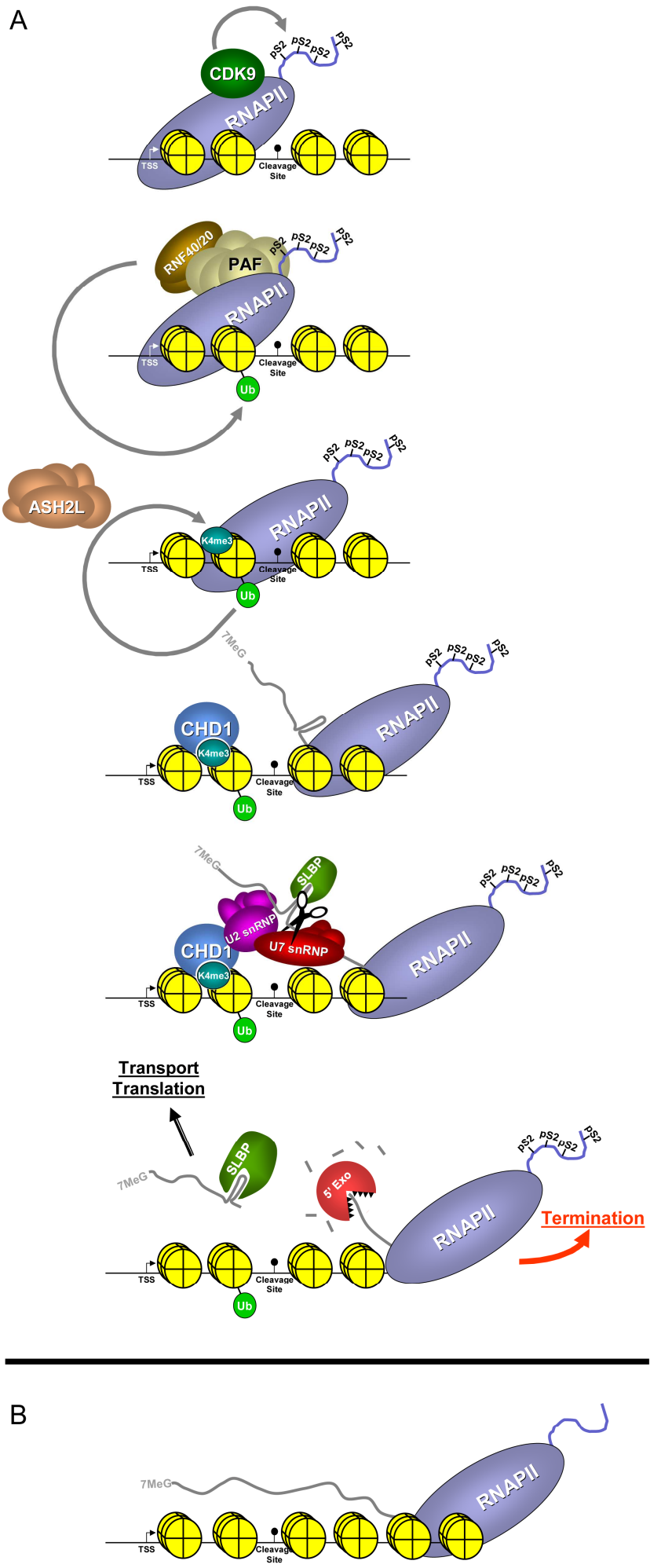

Fig II.3: Schematic representation of the role of CDK9 in controlling replication-dependent histone mRNA 3' end processing. (A) CDK9 recruitment to the HISTIH2BD gene increases the phosphorylation of Ser2 on the RNAPII-CTD which is essential for the recruitment of the PAF complex and histone H2B monoubiquitination by the RNF20/40 ubiquitin ligase complex. H2Bub1 further influences the recruitment of the ASH2L complex. Methylation of H3K4 by the ASH2L complex serves as a molecular landmark allowing for binding of CHD1 which further recruits the U2 snRNP which functions together with the U7 snRNP and SLBP 3' end processing complex to induce transcriptional termination and prevent transcriptional readthrough. (B) The loss of CDK9 activity results in a decrease in H2Bub1 presence on the HISTIH2BD gene. As a result of the loss of H2Bub1, the proper 3' end cleavage site is not recognized and RNAPII continues transcribing until it reaches a downstream polyadenylation signal. 


\subsection{Publication III}

\section{Induced $G_{1}$ cell-cycle arrest controls} replication-dependent histone mRNA 3' end processing through p21, NPAT and CDK9

\section{Citation}

Judith Pirngruber and Steven A. Johnsen

Induced $\mathrm{G}_{1}$ cell cycle arrest controls replication-dependent histone mRNA 3' end processing through $\mathrm{p} 21, \mathrm{NPAT}$ and CDK9

Oncogene (2010), Epub ahead of print

doi:10.1038/onc.2010.42

\section{Own contribution}

Preparation and accomplishment of all biological experiments, i.e. inhibitor treatments, transfection of described siRNAs and constructs, western blot analysis, gene expression analysis, chromatin immunoprecipitation (ChIP), real-time PCR, flow cytometry, apoptose assay, BrdU/PI staining and analysis, cloning of NPAT constructs, GSTpulldown, co-immunoprecipitaion, immunofluorescence and konfocal microscopy; complete figure layout, tables, supplemental materials and methods; contribution to the writing of the manuscript (parts of introduction, methods and results section). 


\begin{abstract}
Proper cell cycle-dependent expression of replication-dependent histones is essential for the packaging of DNA into chromatin during replication. We previously showed that cyclin-dependent kinase-9 (CDK9) controls histone H2B monoubiquitination (H2Bub1) to direct the recruitment of specific mRNA 3' end processing proteins to replication-dependent histone genes and promote proper premRNA 3' end processing. We now show that p53 decreases the expression of the histone-specific transcriptional regulator Nuclear Protein, Ataxia-Telangiectasia Locus (NPAT) by inducing a $\mathrm{G}_{1}$ cell-cycle arrest, thereby affecting E2F-dependent transcription of the NPAT gene. Furthermore, NPAT is essential for histone mRNA 3' end processing and recruits CDK9 to replication-dependent histone genes. Reduced NPAT expression following p53 activation or small interfering RNA knockdown decreases CDK9 recruitment and replication-dependent histone gene transcription but increases the polyadenylation of remaining histone mRNAs. Thus, we present evidence that the induction of a $G_{1}$ cell-cycle arrest (for example, following p53 accumulation) alters histone mRNA 3' end processing and uncover the first mechanism of a regulated switch in the mode of pre-mRNA 3' end processing during a normal cellular process, which may be altered during tumorigenesis.
\end{abstract}

\title{
Introduction
}

Histone protein synthesis is regulated both transcriptionally and posttranscriptionally and is essential for the proper packaging of newly synthesized DNA into chromatin during S phase (Marzluff et al., 2008). Unlike most RNA Polymerase II transcribed mRNAs, replication-dependent histone mRNAs are not polyadenylated but instead end in a conserved 3' stem-loop structure, which is recognized and cleaved by the stem-loop binding protein and the U7 snRNP complexes, respectively (Marzluff et $a l ., 2008)$. It is surprising that the loss of normal histone pre-mRNA processing can result in the production of polyadenylated mRNAs from the replication-dependent histone genes (Narita et al., 2007; Wagner et al., 2007). The levels of polyadenylated canonical histone mRNAs are very low in proliferating cells (Narita et al., 2007; Pirngruber et al., 2009b) but may increase during terminal differentiation (Collart et al., 1991; Kirsh et al., 1989) or during tumorigenesis (Abba et al., 2005; Martinez et al., 2007; Zhao et al., 2004), and opens the possibility that these transcripts may serve an 
important physiological role. However, the mechanisms regulating the switch from canonical histone pre-mRNA processing to polyadenylation remain elusive.

Nuclear Protein, Ataxia-Telangiectasia Locus (NPAT/p220) stimulates the transcription of all replication-dependent histone genes during $\mathrm{S}$ phase (Gao et al., 2003; Ye et al., 2003) in a phosphorylation-dependent manner (Ma et al., 2000; Zhao et al., 1998; Zhao et al., 2000). NPAT functions, in part, by recruiting coactivator proteins including histone-modifying enzymes (DeRan et al., 2008). Moreover, NPAT transcription is regulated in a cell cycle-dependent manner by the E2F family of transcription factors (Gao et al., 2003). However, no role for NPAT in histone mRNA 3 ' end processing has been reported.

Mutation or deletion of the tumor suppressor gene $p 53$ is one of the most common genetic aberrations during tumorigenesis. One of the essential roles in the tumor suppressor function of p53 is the regulation of cell-cycle progression. Most notably, accumulation of the p53 protein can induce a $\mathrm{G}_{1}$ cell-cycle arrest by directly binding and transcriptionally activating the expression of the $p 21$ cyclin-dependent kinase $(\mathrm{CDK})$ inhibitor gene. p21 functions by decreasing cell cycle-regulated CDK activity, which in turn decreases the phosphorylation of retinoblastoma protein (RB) family members and ultimately E2F transcriptional activity. Maintaining an intact p21/RB pathway has been shown to be essential for a significant amount of transcriptional repression elicited by p53 (Lohr et al., 2003). Although p53-induced cell-cycle arrest would be expected to affect replication-dependent histone gene expression, whether this regulation occurs through the $\mathrm{p} 21 / \mathrm{CDK} / \mathrm{RB} / \mathrm{E} 2 \mathrm{~F}$ pathway, whether NPAT gene expression is regulated by this pathway and whether these have functional consequences for replication-dependent histone pre-mRNA processing remain unknown.

Recent studies have uncovered a connection between chromatin and RNA processing. For example, the chromodomain protein CHD1 binds H3K4me3 and is essential for proper mRNA splicing (Sims III et al., 2007), while the variant histones $\mathrm{H} 2 \mathrm{Av}$ and H3.3 control replication-dependent histone pre-mRNA 3' end processing in Drosophila (Wagner et al., 2007). We uncovered a role for CDK9 and specific histone modifications including H2B monoubiquitination (H2Bub1) in controlling replicationdependent histone pre-mRNA 3' end processing in mammalian cells (Pirngruber et al., 2009b; Pirngruber et al., 2009a). In both Drosophila and human cells, interference with chromatin structure resulted in the production of polyadenylated histone mRNAs from 
the replication-dependent histone genes. However, how these processes are regulated and whether the observed shift in the mode of pre-mRNA 3' end processing occurs under normal cellular processes remains unknown. In this study, we identified a reciprocal regulation of replication-dependent histone gene transcription and mRNA polyadenylation in which the induction of a $G_{1}$ cell-cycle arrest following p53 accumulation resulted in the production of polyadenylated transcripts. This effect was recapitulated by the induction of quiescence following the removal of serum, after treatment with hydroxyurea or after knockdown of cyclin E1. This regulation was further investigated and found to be dependent on p53-induced expression of the CDK inhibitor p21, a decrease in NPAT gene expression caused by increased binding of the E2F4/p130 repressor complex to the NPAT promoter, and decreased recruitment of NPAT, CDK9 and polymerase associated factor-1 (PAF1) to endogenous replicationdependent histone genes.

\section{Results}

\section{P53 regulates histone pre-mRNA 3' end processing}

Gene expression microarray studies are typically based on an initial polyTprimed reverse transcription step that specifically labels polyadenylated mRNAs. A number of microarray studies identified changes in the expression of replicationdependent histone genes, thus uncovering potential regulatory pathways controlling histone pre-mRNA 3' end processing. One study identified a correlation between replication-dependent histone mRNA polyadenylation and p53 status in head and neck squamous cell carcinomas (Yan et al., 2007), while another study identified a downregulation in the expression of polyadenylated replication-dependent histone mRNAs in human papilloma virus-positive (HPV+) squamous cell carcinoma of the head and neck (Martinez et al., 2007). As p53 is a primary target of the HPV E6 oncoprotein (zur Hausen and de Villiers, 1994), it is possible that the changes observed in the second study were also due to regulation of p53 function. Therefore, we tested whether the accumulation of p53 affects replication-dependent histone pre-mRNA 3' end processing by treating wild-type (p53+/+), p53 null (p53-/-) or p21 null (p21-/-) HCT116 cells with Nutlin-3a, which increases endogenous p53 protein levels by blocking the interaction with its negative regulator murine double minute 2 (MDM2). Consistent with the role of p53 and its downstream target gene, the CDK inhibitor p21, 
in controlling cell-cycle progression, Nutlin-3a treatment increased the fraction of cells in the G1 cell-cycle phase (Fig III.1A), decreased RB phosphorylation (Fig III.1B) and decreased total replication-dependent histone gene expression (HIST1H2BD and HIST1H2AC) in a p53- and p21-dependent manner (Supp Fig III.S1 and Fig III.1C,D, left panel). As reported by others (Carvajal et al., 2005; Kranz and Dobbelstein, 2006),

A
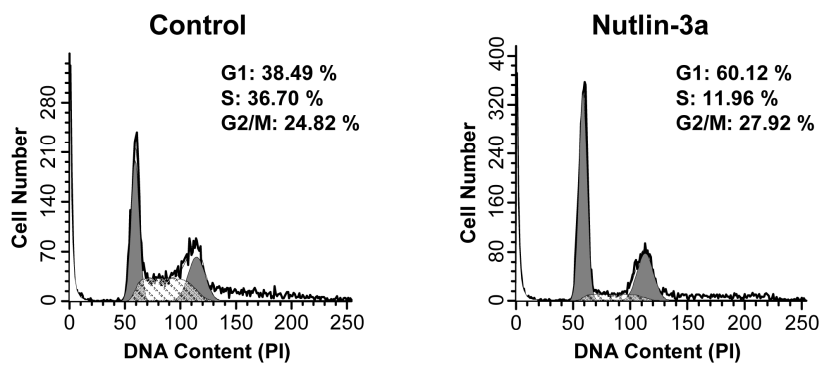

B

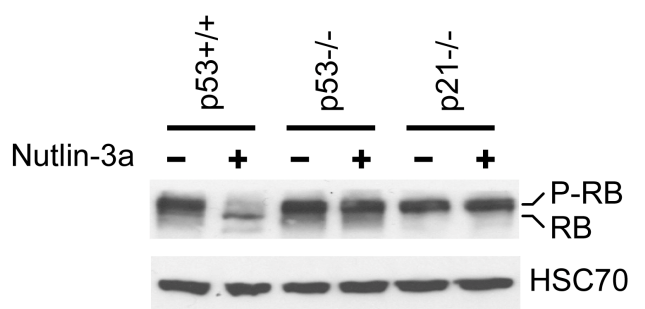

C

$\square$ Control

Nutlin-3a
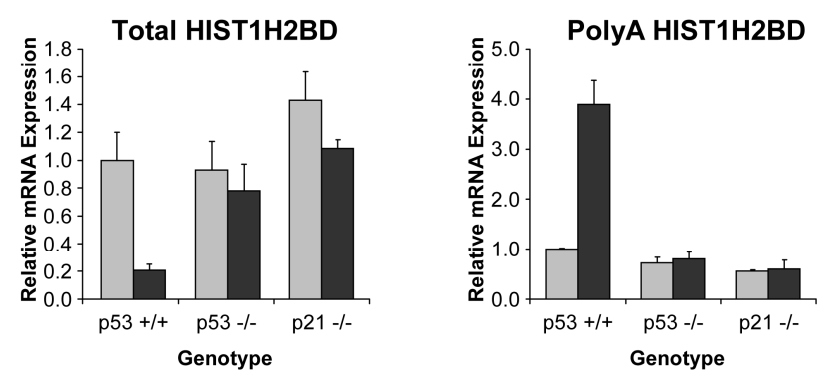

D
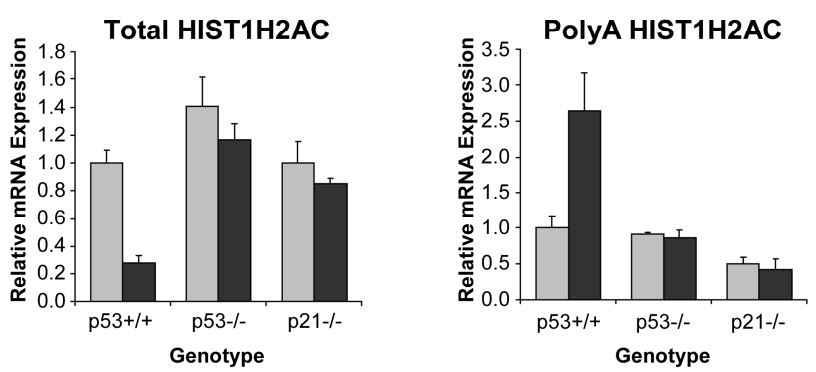

$\mathrm{E}$
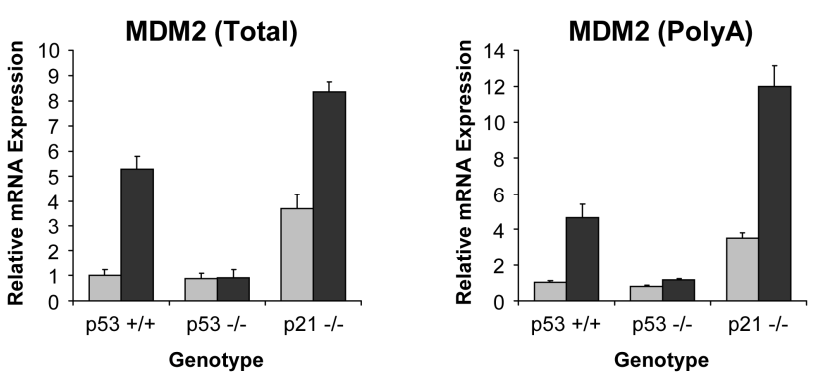
Fig III.1: p53 accumulation reciprocally regulates replication-dependent histone gene expression and mRNA polyadenylation in a p21-dependent manner. (A) HCT116 cells were treated with $8 \mu \mathrm{M}$ Nutlin-3a for $24 \mathrm{~h}$ and analyzed by flow cytometry for cell-cycle analyses. (B) HCT116 cells with the indicated p53 and p21 genotypes were treated as in (A) and analyzed by western blot for RB expression and phosphorylation using an antibody that detects total RB. HSC70 is shown as a loading control. (C-E) Total RNA from HCT1116 cells with the indicated genotypes that were either control (dimethyl sulfoxide) or Nutlin-3a treated were analyzed by random- (left panels) or oligo-dT-primed (right panels) quantitative reverse transcriptase PCR for the expression of total or polyadenylated HIST1H2BD (C), HIST1H2AC (D) and MDM2 (E). Gene expression was normalized to an unregulated gene, RPLP0, and the control condition, and is represented as relative mRNA expression; mean values $+\mathrm{s} . \mathrm{d}$., $n=3$.

we observed no significant increase in the percentage of apoptotic cells following Nutlin-3a treatment of HCT116 cells for 24 h (Supp Fig III.S2). In contrast to total replication-dependent histone gene expression, the levels of polyadenylated HIST1H2BD and HIST1H2AC increased following Nutlin-3a treatment (Fig III.1C,D, right panel). Taking the downregulation of total mRNA levels into account, these results reflect approximately 20 - and 10-fold increases in the polyadenylated fractions of HIST1H2BD and HIST1H2AC mRNAs, respectively. The p21 dependence was not due to a loss of p53 transcriptional activity, as the induction of the negative feedback regulator MDM2 was maintained in the absence of p21 (Fig III.1E). Furthermore, as expected, owing to a decreased need for new histone protein production in nonproliferating cells, no changes in overall histone protein levels were detected (Supp Fig III.S3).

\section{P53 accumulation affects CDK9 and PAF1 recruitment}

On the basis of our previous studies (Pirngruber et al., 2009b), we hypothesized that the effects of p53 accumulation on histone pre-mRNA 3' end processing may be due to changes in the recruitment of CDK9 and PAF1, which are both essential for $\mathrm{H} 2 \mathrm{~B}$ monoubiquitination and maintaining normal histone pre-mRNA 3' end processing. We therefore performed chromatin immunoprecipitation (ChIP) analyses using control or Nutlin-3a-treated wild-type HCT116 cells. Consistent with our hypothesis, CDK9 and PAF1 were preferentially localized to the 3 ' end cleavage site $(0.5 \mathrm{~kb})$, and their levels at both the transcriptional start site (TSS) and $0.5 \mathrm{~kb}$ decreased following Nutlin-3a treatment (Fig III.2A,B). All factors were absent from an unrelated intergenic region close to the trefoil factor-1 (TFF1) gene, which is silent in estrogen receptor-negative cell lines including HCT116 (Fig III.2 and data not shown). 
A

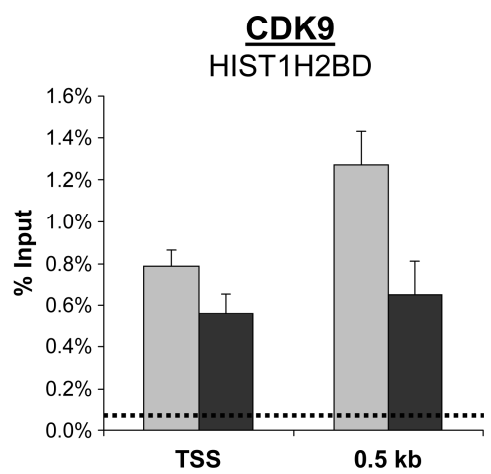

B

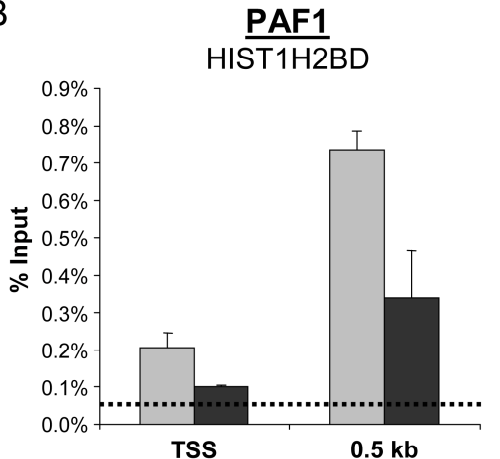

C

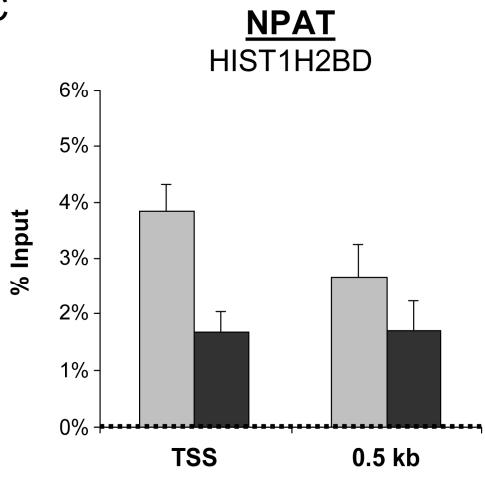

$\square$ Control

Nutlin-3a
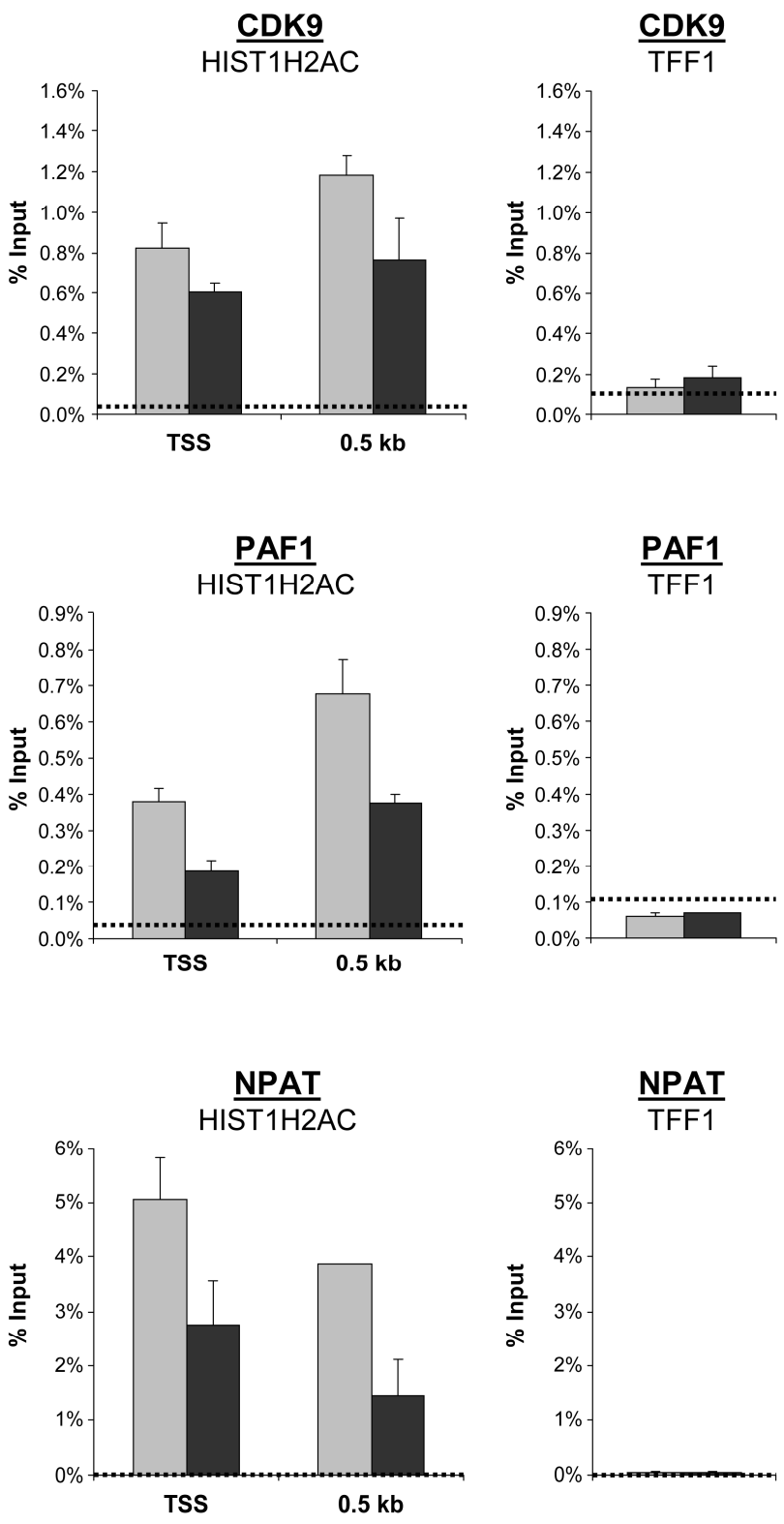

Fig III.2: Accumulation of p53 decreases the recruitment of CDK9, PAF1 and NPAT. ChIP analysis of the HISTIH2BD (left panels), HISTIH2AC (middle panels) genes or an intergenic locus close to the TFF1 gene (right panels) was performed using the indicated antibodies with extracts from control or Nutlin-3a treated HCT116 cells. CDK9 (A) and PAF1 (B) are preferentially recruited to the 3' end processing site and their recruitment to the TSS and $0.5 \mathrm{~kb}$ sites on both histone genes is decreased following Nutlin-3a treatment. (C) The histone-specific transcriptional cofactor NPAT is recruited to both the TSS and $0.5 \mathrm{~kb}$ of both histone genes and its levels decrease following Nutlin-3a treatment. All proteins were absent from the negative control (TFF1) locus (A-C, right panel). qChIP values were normalized to their respective DNA inputs and expressed as DNA recovery (percentage input); mean values + s.d., $n=3$. The experimental background (control $\operatorname{IgG}$ ) is shown as a dotted line. 


\section{Decreased NPAT recruitment to the TSS and $0.5 \mathrm{~kb}$ following $\mathrm{p53}$ accumulation}

Given the role for NPAT in controlling replication-dependent histone expression, we also examined its recruitment to two replication-dependent histone genes (HIST1H2BD and HIST1H2AC) following Nutlin-3a treatment. It is surprising that despite its reported role as a site-specific transcriptional cofactor, we detected NPAT at both the TSS and $0.5 \mathrm{~kb}$ regions of the histone genes, indicating a potential role in histone pre-mRNA 3' end processing (Fig III.2C). Importantly, NPAT recruitment to these genes was decreased at both positions following Nutlin-3a treatment.

\section{Cell-cycle and histone mRNA polyadenylation}

The correlation between p53-induced cell-cycle arrest and the changes in histone mRNA expression and polyadenylation prompted us to perform time course analyses following Nutlin-3a treatment. Total replication-dependent histone mRNA levels already dropped at $8 \mathrm{~h}$ after treatment, which preceded an increase in polyadenylated mRNAs (Fig III.3A). The time-dependent decrease in total histone mRNA levels correlated with decreases in NPAT mRNA levels (Fig III.3B) and the fraction of cells in S phase (Fig III.3D, Supp Fig III.S4). These effects were preceded by an increase in p21 mRNA levels (Fig III.3C), which also preceded an increase in the fraction of cells in $\mathrm{G}_{1}$ phase (Fig III.3D).

Additional protein expression analyses supported that p53 accumulation correlates with the induction of p21 mRNA levels $(4 \mathrm{~h})$ and precedes maximal p21 protein levels $(8 \mathrm{~h})$, which in turn precede a decrease in NPAT expression and phosphorylation $(16 \mathrm{~h})$ and ultimately a dramatic decrease in total NPAT protein levels (24 h) (Fig III.3E). In contrast, CDK9 and cyclin T1 levels were unaffected by Nutlin3a treatment. Importantly, and consistent with the role of p21 in controlling E2F and RB activity, the effects of Nutlin-3a on NPAT expression are dependent upon both p53 and p21 (Supp Fig III.S5A).

The induction of cell-cycle arrest results in a decrease in E2F transcriptional activity caused by changes in the complex formation between specific E2F and RB family members and their recruitment to E2F target genes (Takahashi et al., 2000). In particular, E2F4 and the RB family member p130 are specifically associated with $\mathrm{G}_{1}$ cell-cycle arrest and quiescence $\left(\mathrm{G}_{0}\right)$ (Vairo et al., 1995). Therefore, as NPAT is an E2F target gene, we tested whether p53-induced $\mathrm{G}_{1}$ cell-cycle arrest also resulted in an increase in E2F4 and p130 binding to the NPAT gene. Indeed, E2F1, E2F4 and p130 
were all detectable on both the NPAT and p107 genes, and the levels of E2F4 and p130 increased following Nutlin-3a treatment (Fig III.3F, Supp Fig III.S5B). All three proteins were not detectable on the TFF1 gene (Supp Fig III.S5B).

As NPAT expression was reported to regulate the formation of subnuclear coilin-containing Cajal bodies (Ye et al., 2003) and ionizing irradiation downregulated NPAT expression and caused a loss of focal nuclear NPAT staining (Su et al., 2004), we also tested whether p53 accumulation affected NPAT localization and Cajal body integrity. Consistent with the western blot results, the number of NPAT foci per cell dropped dramatically to nearly background levels following Nutlin-3a treatment, whereas Cajal body structure was maintained (Fig III.3G, Supp Fig III.S5C). However, the overall colocalization frequency of NPAT to Cajal bodies was not affected by Nutlin-3a treatment since 79.6 and $80.0 \%$ of NPAT foci colocalized with Coilin in the presence or absence of Nutlin-3a, respectively $(n=23)$. Thus, p53 accumulation specifically affects histone locus body formation by NPAT without affecting overall Cajal body structure.

\section{Increased histone $m R N A$ polyadenylation is a result of $G_{1}$ cell-cycle arrest}

To determine whether the effects of Nutlin-3a treatment on histone mRNA polyadenylation were a specific effect of p53 accumulation or a more general consequence of $\mathrm{G}_{1}$ cell-cycle arrest, we induced quiescence in HCT116 cells by serum starvation. As expected, serum starvation substantially increased the $\mathrm{G}_{0} / \mathrm{G}_{1}$ cell fraction (Fig III.4A). Similar to Nutlin-3a treatment, serum starvation also increased histone

Fig III.3: Accumulation of p53 and induction of p21 precede cell-cycle arrest, decreases in replication-dependent histone and NPAT gene expression, and ultimately accumulation of polyadenylated histone transcripts. (A) The expression of total and polyadenylated HIST1H2BD was analyzed as in Fig III.1C at the indicated times following Nutlin-3a treatment. The expression of NPAT (B) and p21 (C) mRNA was analyzed by random-primed quantitative reverse transcriptase PCR as performed for total HIST1H2BD in (A). Gene expression levels were normalized and expressed as in Fig III.1; mean values + s.d., $n=2$. (D) Combined propidium iodide/5-bromodeoxyuridine staining (left panel) or propidium iodide alone (right panel) were performed as in Fig III.1A to determine the kinetic induction of cell cycle-arrest following Nutlin-3a treatment. The percentage of cells in the $S$ and $G_{1}$ phases is indicated. (E) Western blot analysis was performed on protein samples prepared in parallel to the samples in (A-D) using the indicated antibodies. HSC70 is shown as a loading control. (F) E2F4 and p130 binding to the NPAT promoter increases following Nutlin-3a treatment. E2F1, E2F4 and p130 recruitment to the NPAT gene was investigated by ChIP analysis. qChIP values were normalized as in Fig III.2; mean values + s.d., $n=3$. The experimental background is shown as a dotted line. (G) Nutlin-3a treatment decreases the number of NPAT foci without affecting the number or intensity of Cajal bodies. HCT116 cells were treated with Nutlin-3a for $24 \mathrm{~h}$ and analyzed by confocal immunofluorescence microscopy using specific antibodies against NPAT (red) or Coilin (green). 4,6-Diamidino-2phenylindole staining (blue) indicates the nuclei of individual cells. The white line in the bottom right corner indicates a size of $10 \mu \mathrm{m}$. 
A

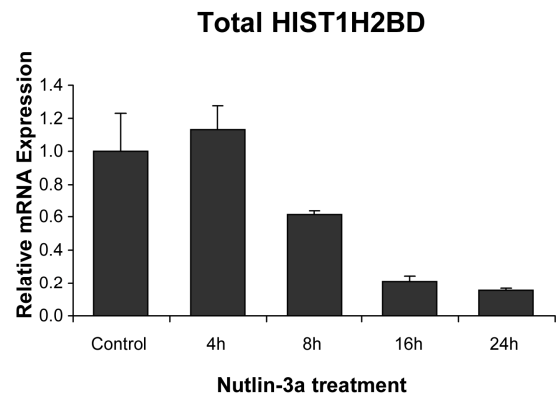

B

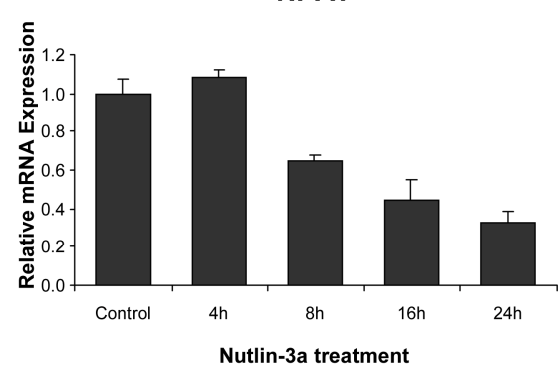

D

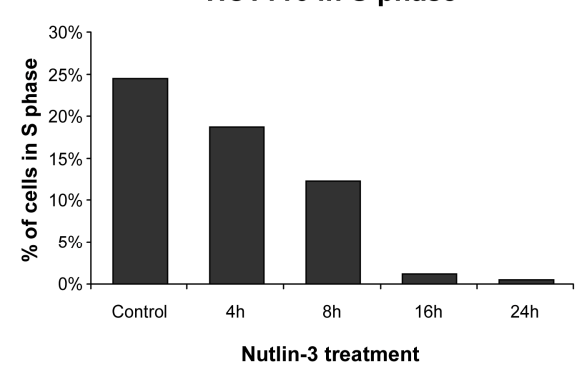

E

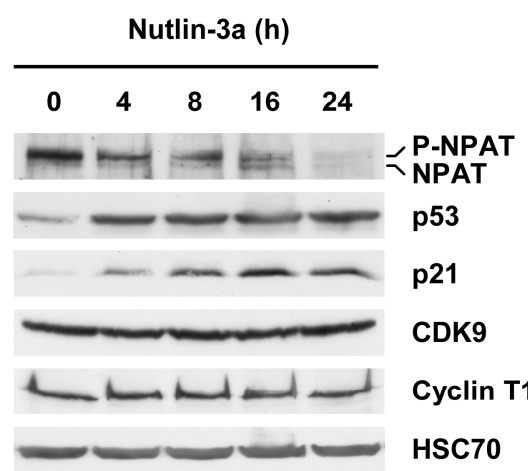

G

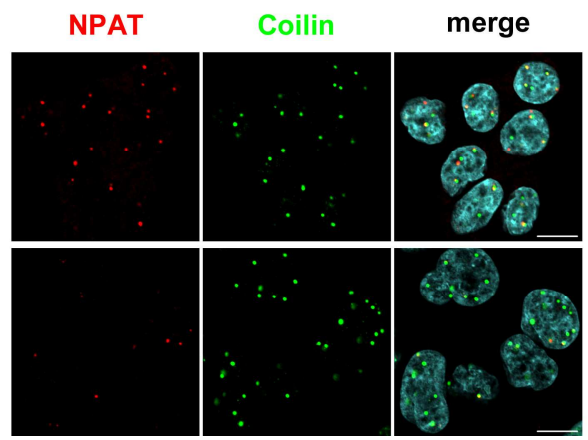

PolyA HIST1H2BD

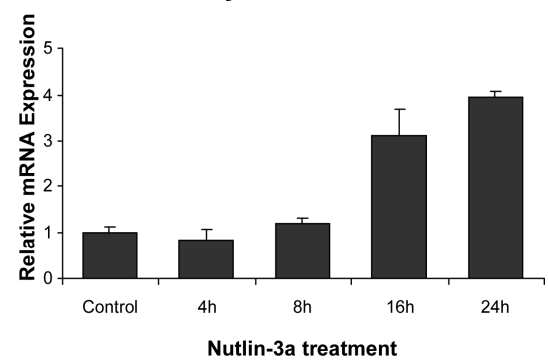

C

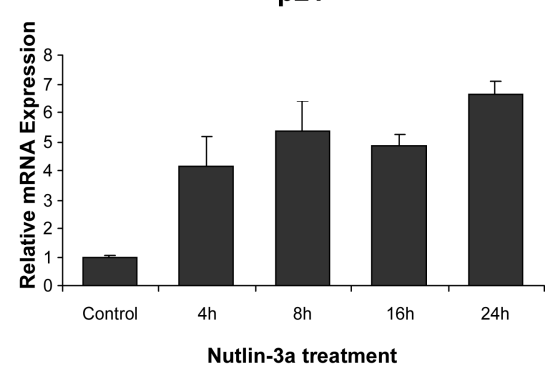

HCT116 in G1 phase

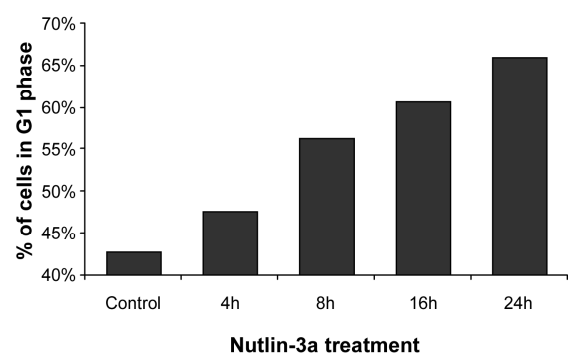

F

NPAT

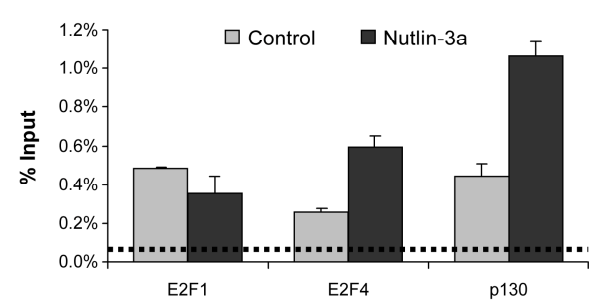

Control

Nutlin-3a 
mRNA polyadenylation (Fig III.4B and Supp Fig III.S6A). Furthermore, we also performed knockdown analyses of the $\mathrm{G}_{1} / \mathrm{S}$ cyclin $\mathrm{E} 1$ (Fig III.4C) and observed decreases in NPAT protein levels (Fig III.4D) and total replication-dependent histone gene expression, but significant increases in polyadenylated histone gene transcripts (Fig III.4E and Supp Fig III.6B). These effects were also independent of p53 status as the knockdown of cyclin E1 in p53 null HCT116 cells also resulted in similar effects on histone gene expression and pre-mRNA 3' end processing as seen in wild-type HCT116 cells (Supp Fig III.S7).

The anti-neoplastic drug hydroxyurea (HU) inhibits DNA replication thereby causing an arrest in $G_{1}$ or early $S$ phase of the cell-cycle and eventually leads to the formation of double-strand breaks and the induction of a DNA damage response (Rappold et al., 2001; Saintigny et al., 2001). Therefore, we also tested whether HU treatment may also affect replication-dependent histone mRNA 3' end processing. Indeed, HU treatment for $48 \mathrm{~h}$ reciprocally decreased total histone mRNA levels and increased polyadenylated mRNA levels irrespective of p53 status (Fig III.4F and Supp Fig III.6C and data not shown).

\section{NPAT regulates histone pre-mRNA 3' end processing}

The presence of NPAT at both the TSS and 3' end processing sites on histone genes, combined with the inverse correlation between NPAT and polyadenylated histone mRNA expression, suggested that NPAT may function to regulate both replication-dependent histone transcription and 3' end processing. Thus, we transfected HCT116 cells with two individual small interfering RNAs (siRNAs) against NPAT and performed gene expression analyses. Consistent with previous reports, NPAT

Fig III.4: Accumulation of polyadenylated replication-dependent histone mRNAs is induced following a $\mathbf{G}_{\mathbf{1}} / \mathbf{G}_{\mathbf{0}}$ cell-cycle arrest. (A) HCT116 cells were grown in serum free medium for $24 \mathrm{~h}$ and cell-cycle distribution was analyzed by propidium iodide-based flow cytometry as in Fig III.1A. (B) Serum starvation downregulates total HIST1H2BD but increases mRNA polyadenylation. Gene expression levels were normalized and expressed as in Fig III.1; mean values + s.d., $n=2$. (C-E) Cyclin E1 knockdown similarly decreases NPAT and replication-dependent histone gene expression and induces histone mRNA polyadenylation. (C) The effectiveness of cyclin E1 knockdown was assessed by randomprimed quantitative reverse transcriptase PCR in HCT116 cells at $52 \mathrm{~h}$ after transfection with control or cyclin E1 siRNAs. (D) Western blot analysis was performed on protein samples prepared in parallel to the samples in (C and E) using NPAT or HSC70 antibodies. HSC70 is shown as a loading control. (E) The expression of total and polyadenylated HIST1H2BD mRNA was assessed as in Fig III.1 using the same RNA samples as in (C). Gene expression levels were normalized and expressed as in Fig III.1; mean values + s.d., $n=2$. (F) The expression of total and polyadenylated HIST1H2BD was analyzed as in Fig III.1C after treatment with $2 \mathrm{mM}$ hydroxyurea for $48 \mathrm{~h}$. Gene expression levels were normalized and expressed as in Fig III.1; mean values + s.d., $n=2$. 
A
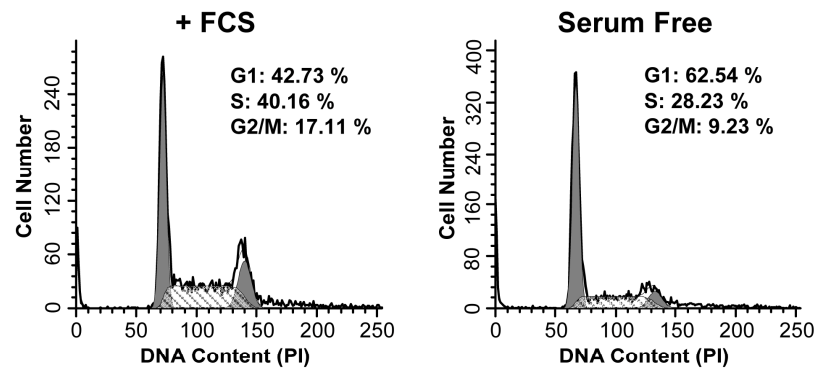

B

$$
\square+\text { FCS } \quad \square \text { Serum Free }
$$

Total HIST1H2BD

PolyA HIST1H2BD
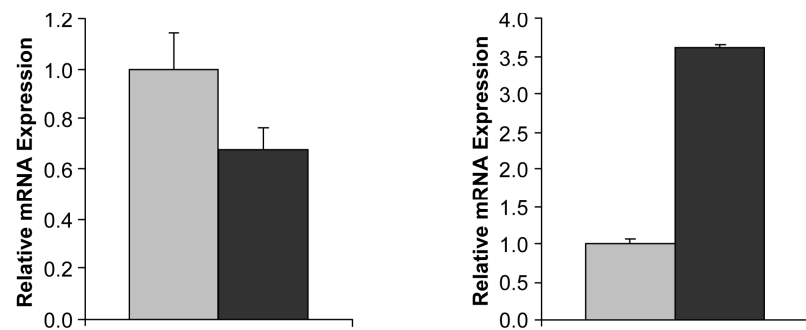

C

$\square$ Control siRNA $\square$ Cyclin E1 siRNA

D SiRNA

Cyclin E1
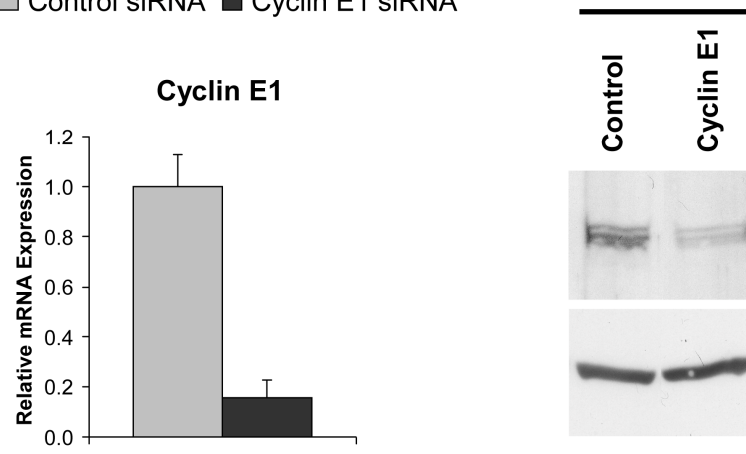

E

$\square$ Control siRNA $\square$ Cyclin E1 siRNA

Total HIST1H2BD

PolyA HIST1H2BD
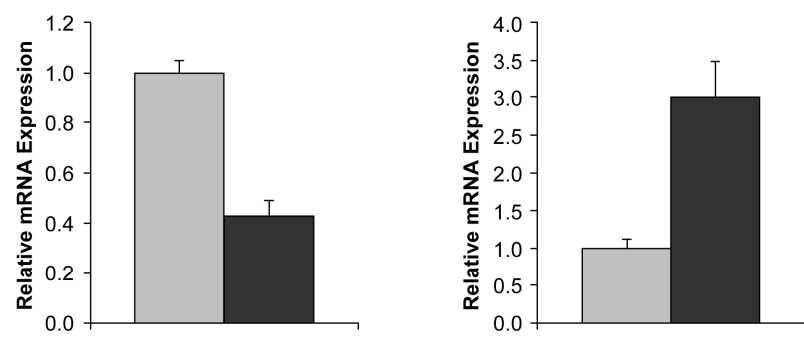

F

$\square$ Control

Hydroxyurea $48 \mathrm{~h}$

Total HIST1H2BD

PolyA HIST1H2BD
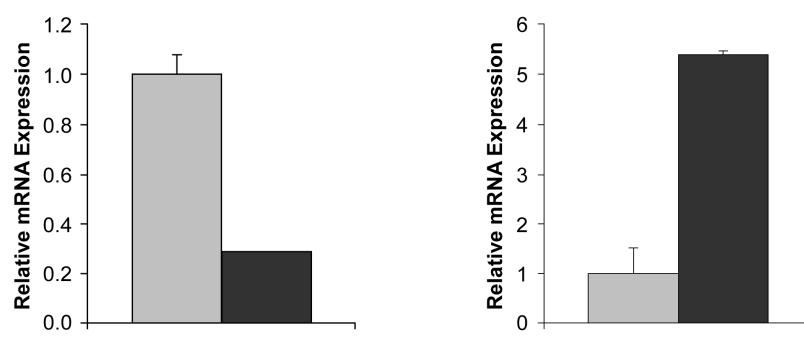
knockdown (Fig III.5C, Supp Fig III.S8C,D) resulted in a decrease in total histone mRNA levels (Fig III.5A,B, Supp Fig III.S8A,B). In contrast, and similar to Nutlin-3a treatment or cyclin E1 knockdown, NPAT knockdown resulted in a dramatic increase in the levels of polyadenylated histone mRNAs (Fig III.5A,B, Supp Fig III.S8A,B) similar to that observed following CDK9 knockdown (Supp Fig III.S8E) (Pirngruber et al., 2009b).

\section{NPAT helps recruit CDK9 to histone genes}

Given the similarities between the effects of Nutlin-3a treatment and NPAT knockdown on histone pre-mRNA $3^{\text {' }}$ end processing and the role of CDK9 in suppressing histone mRNA polyadenylation, we tested whether NPAT and the CDK9/cyclin T1 complex interact in vitro using glutathione $S$-transferase (GST) pulldown analyses. On the basis of the presence of an RXL cyclin-binding motif at positions 1062-1066 of the NPAT protein (Wei et al., 2003) and a previous report indicating that another RXL domain directly interacts with a CDK9 complex (Simone et al., 2002a), we tested whether this region (amino acids 801-1100) of NPAT may also interact with the CDK9/cyclin T1 complex. Indeed, as shown in Fig III.5D, the GSTNPAT $^{801-1100}$ fusion protein, but not GST alone, was capable of pulling down both cyclin T1 and CDK9.

To verify this interaction in vivo, we performed co-immunoprecipitation analyses with antibodies against CDK9 and NPAT. Indeed, immunoprecipitation of CDK9 resulted in a specific co-immunoprecipitation of NPAT (Fig III.5E). Similarly, immunoprecipitation of NPAT also co-immunoprecipitated cyclin T1 and CDK9 as detected with two separate CDK9 antibodies.

We further tested whether the NPAT-CDK9 interaction is of functional consequence by performing ChIP analyses on two histone genes and a control locus following transfection with either control or NPAT siRNAs. As observed following Nutlin-3a treatment, NPAT knockdown resulted in decreases in both CDK9 and PAF1 recruitment (Fig III.6A,B). These effects were most pronounced at $0.5 \mathrm{~kb}$, consistent with the roles of NPAT, CDK9 and PAF1 in controlling 3' end processing. The effectiveness of the NPAT knockdown was verified by ChIP against NPAT (Fig III.6C). These effects were also specific for NPAT knockdown, as the transfection of two different control siRNAs did not appreciably affect CDK9, PAF1 or NPAT recruitment compared with mock-transfected cells (Supp Fig III.S9A-C). 
A

$\square$ Control siRNA

Total HIST1H2BD

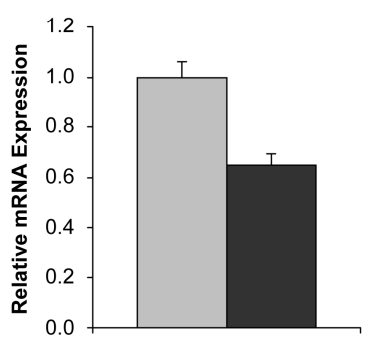

B

Total HIST1H2AC

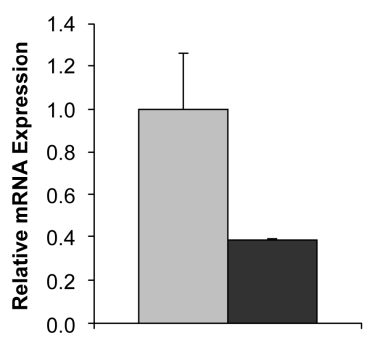

C

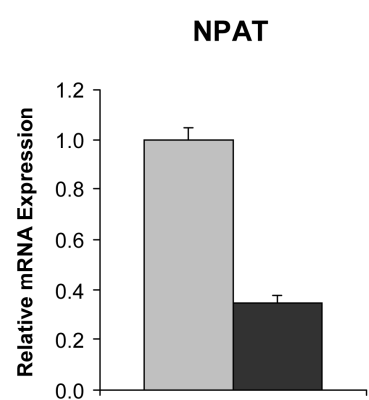

E

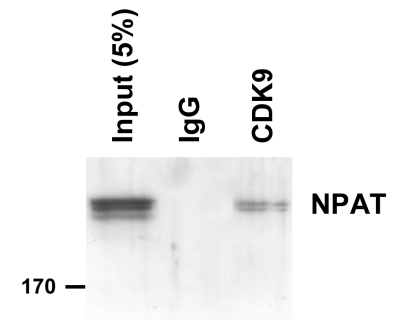

NPAT SIRNA

PolyA HIST1H2BD

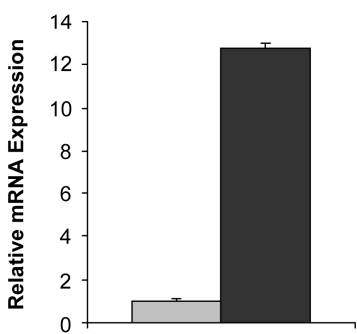

PolyA HIST1H2AC
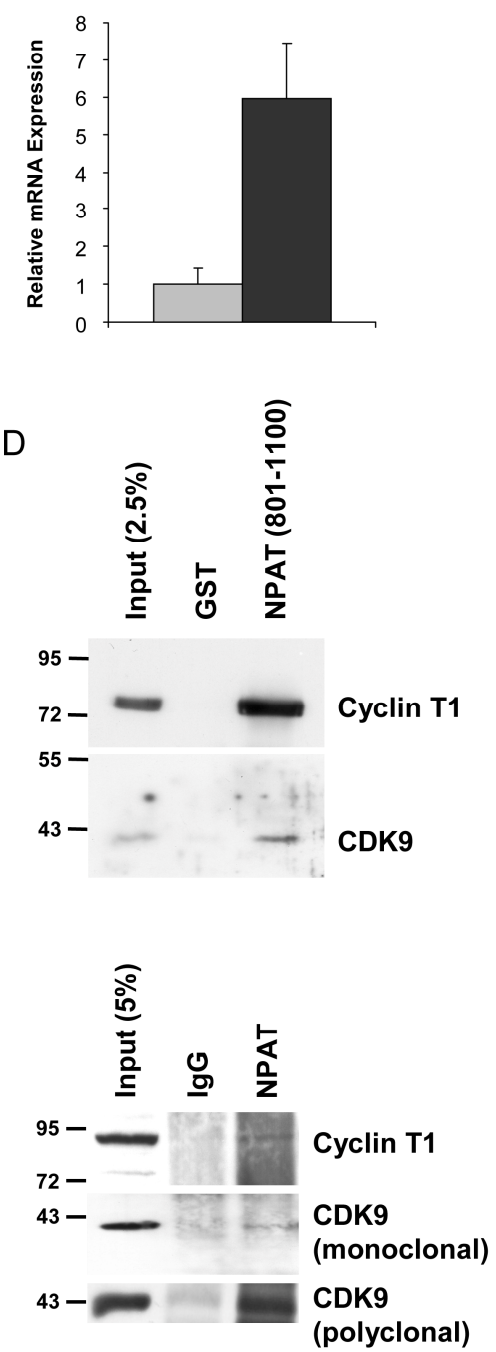

Fig III.5: NPAT expression is essential for optimal levels of replication-dependent gene transcription and proper pre-mRNA 3 ' end processing. (A,B) The expression of total and polyadenylated HIST1H2BD (A) and HIST1H2AC (B) mRNAs was assessed in HCT116 cells $72 \mathrm{~h}$ after transfection with control or NPAT siRNAs as in Fig III.1. (C) The effectiveness of NPAT knockdown was assessed by random-primed quantitative reverse transcriptase PCR using the same RNA samples as in (A). Gene expression levels were normalized and expressed as in Fig III.1; mean values + s.d., $n=2$. (D,E) Complex formation between NPAT and CDK9/cyclin T1. (D) in vitro GST pull-down analysis was performed using in vitro transcribed and translated P-TEFb (CDK9/cyclin T1) as a bait for bacterially expressed and purified GST or GST-NPAT ${ }^{801-1100}$, which contains the RXL cyclin-binding motif. (E) HCT116 cell lysates were immunoprecipitated with nonspecific $\mathrm{IgG}$ or antibodies against endogenous NPAT and CDK9. Endogenous co-immunoprecipitated proteins were revealed by western blot analysis against CDK9, cyclin T1 and NPAT. 


\section{Discussion}

The induction of p53-dependent cell-cycle arrest has an important role in the control of cellular proliferation and may help protect cells against the effects of certain chemotherapeutic agents (Carvajal et al., 2005; Kranz and Dobbelstein, 2006). The induction of polyadenylated mRNAs produced from replication-dependent histone genes following $p 53$-induced $G_{1}$ cell-cycle arrest may be significant as the polyadenylated histone transcripts have a longer half-life than the normally processed transcripts from the same genes (Kirsh et al., 1989) and are translated in a cell cycleindependent manner. This may be important, for example, in quiescent or terminally differentiated cells in which the, albeit lower, levels of polyadenylated histone mRNAs (compared with non-polyadenylated expressed during $\mathrm{S}$ phase) would serve to maintain proper DNA packaging and chromatin structure in the absence of DNA replication. Although the existence of polyadenylated canonical histone transcripts produced from replication-dependent histone genes has been previously reported in mammalian cells (Kirsh et al., 1989; Narita et al., 2007; Pirngruber et al., 2009b), the mechanisms that control when and how these transcripts are produced remained a mystery. Our data provide the first evidence that the induction of a $G_{1}$ cell-cycle arrest following p53 accumulation, serum withdrawal, cyclin E1 knockdown or HU treatment leads to an increase in the production of polyadenylated histone transcripts produced from canonical replication-dependent histone genes. We have shown that in the case of Nutlin-3a treatment this regulation is dependent upon both p53 and its downstream target p21. Our results suggest a time-dependent model in which p53 induction leads to an increase in p21 gene expression. This in turn causes an accumulation of cells in the $\mathrm{G}_{1}$ phase and leads to a decrease in E2F transcriptional activity, which ultimately decreases NPAT gene expression. We have shown that NPAT forms a complex with CDK9 and cyclin T1 and thereby likely functions to modulate chromatin modifications through the recruitment of the RNA polymerase II-associated PAF complex. On the basis of these data and the model we previously described (Pirngruber et al., 2009b; Pirngruber et al., 2009a), we propose that NPAT gene expression during normal cellcycle progression leads to the recruitment of the P-TEFb complex (CDK9/Cyclin T1; Fig III.6D), thereby facilitating PAF1 recruitment and histone H2B monoubiquitination (not shown). This in turn increases both the transcription and efficiency of canonical histone mRNA 3' end processing. In contrast, during $G_{1} / G_{0}$ cell-cycle arrest, NPAT expression is decreased and P-TEFb is less efficiently recruited (Fig III.6E). This results 
A

$\square$ Control siRNA $\square$ NPAT siRNA \#1
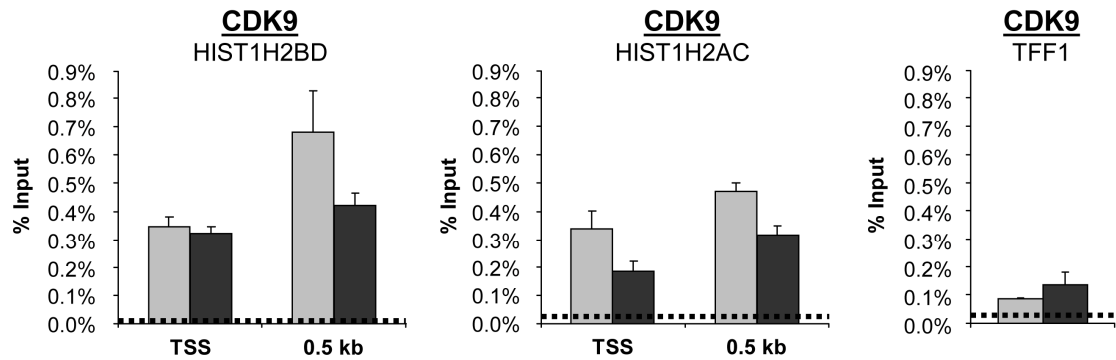

B
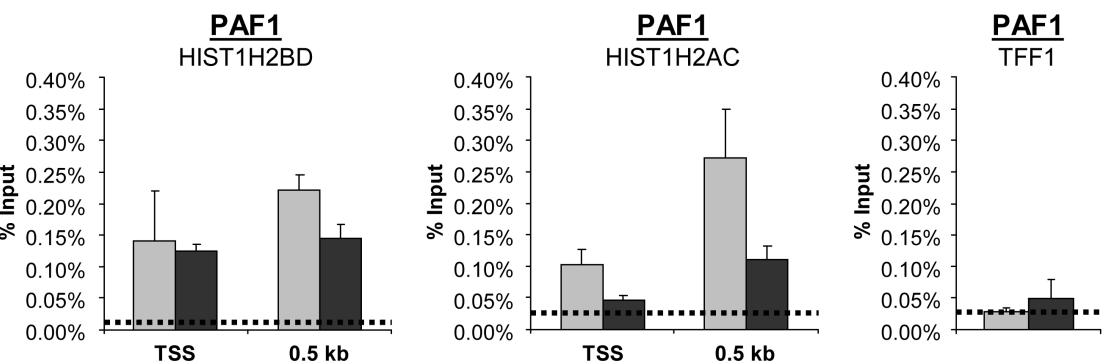

C
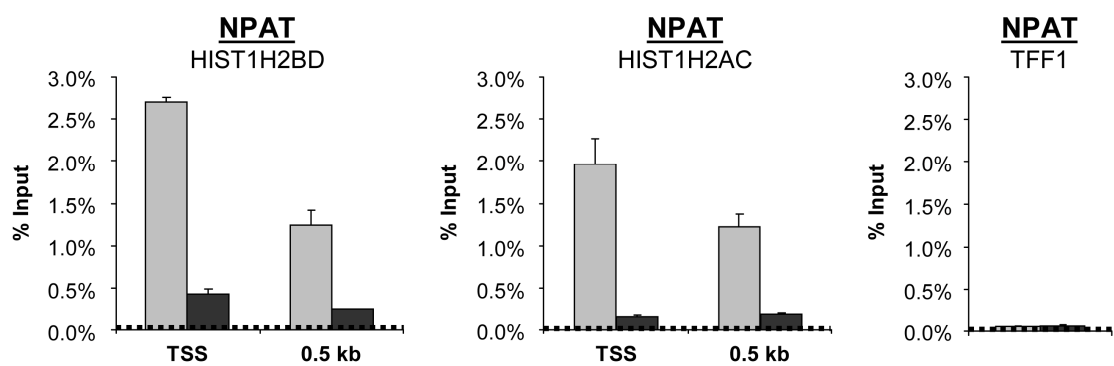

D

\section{Normal cell cycle progression}

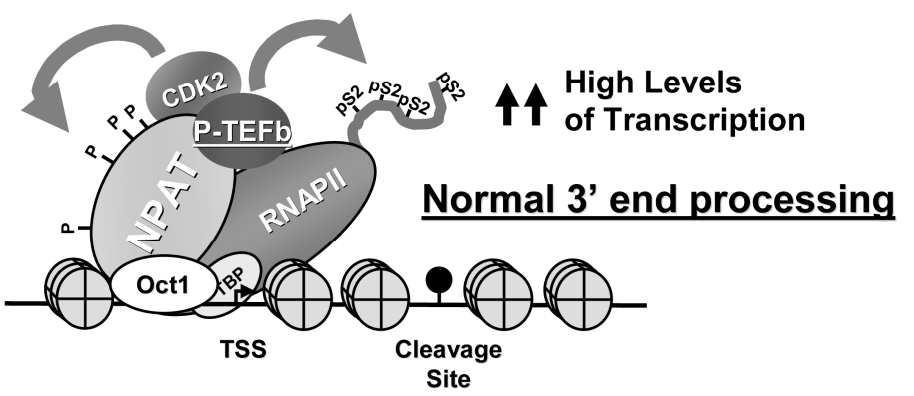

$E$

Following G1/G0 cell cycle arrest

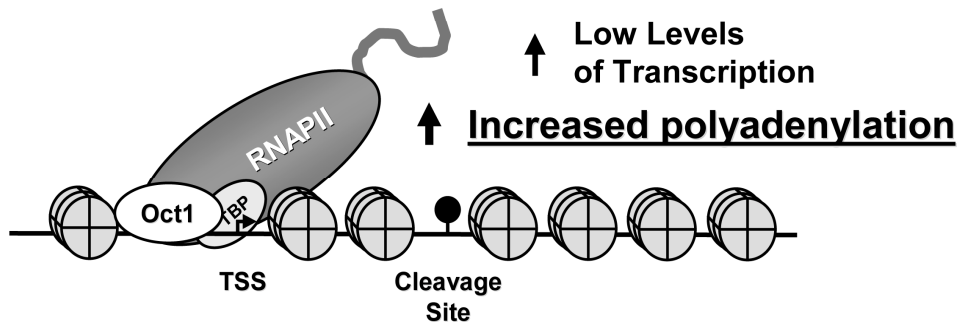


Fig III.6: NPAT knockdown mimics p53-induced $G_{1}$ cell-cycle arrest and is required for optimal recruitment of CDK9 and PAF1. Protein recruitment to a negative control locus (TFF1) and the TSS and $0.5 \mathrm{~kb}$ regions of HISTIH2BD and HIST1H2AC was investigated by ChIP analysis as in Fig III.2 using antibodies against CDK9 (A), PAF1 (B) and NPAT as a knockdown control (C). qChIP values were normalized as in Fig III.2; mean values + s.d., $n=3$. The experimental background (control IgG) is shown as a dotted line. (D,E) Proposed model of NPAT/P-TEFb interactions in controlling the mode of replication-dependent histone mRNA transcription and 3' end processing. (D) During normal cell-cycle progression NPAT is expressed in an E2F-dependent manner and recruited to histone genes through other factors such as Oct1 and is activated through phosphorylation by CDK2/cyclin E1. NPAT binding increases $\mathrm{P}-\mathrm{TEFb}$ (CDK9/cyclin T1) recruitment, thereby increasing histone gene transcription and canonical 3' end processing through increased RNA polymerase II CTD phosphorylation and subsequent recruitment of PAF1 and histone $\mathrm{H} 2 \mathrm{~B}$ monoubiquitination. (E) Owing to the decreased expression of NPAT during $\mathrm{G}_{1} / \mathrm{G}_{0}$ cell-cycle arrest, less P-TEFb is recruited to the replication-dependent histone genes, thereby decreasing overall transcription and increasing the frequency of histone mRNA polyadenylation.

in decreased overall levels of replication-dependent histone gene transcription but an increase in cell cycle-independent 3' end processing (polyadenylation).

The correct packaging of DNA into chromatin is essential for maintaining genomic integrity and structure during normal cell-cycle progression. To maintain this structure, cells use a number of intricate mechanisms to control histone gene expression including gene transcription, pre-mRNA processing, mRNA transport, translation and degradation. With the exception of histone gene expression, most of the remaining regulatory steps depend upon the recognition of the unique 3' end stem-loop structure. Therefore, the expression of alternative 3' end-processed (polyadenylated) mRNAs produced from normally replication-dependent histone genes may be a previously unrecognized, but relatively simple, mechanism of producing canonical histones outside the $\mathrm{S}$ phase. This extra source of $\mathrm{S}$ phase-independent histones may be advantageous for quiescent, terminally differentiated cells or tumor cells. Consistent with a potential positive role of polyadenylated histone mRNAs during tumorigenesis, the overexpression of a transgene that produces a polyadenylated mRNA encoding a canonical core histone was sufficient to increase epidermal growth factor-induced cellular proliferation (Choi et al., 2005). Furthermore, the expression of polyadenylated canonical histone mRNAs may be important for cells experiencing DNA damage. The polyubiquitination and exchange of core histones following DNA damage is essential for the cellular DNA damage response (Huen et al., 2007; Mailand et al., 2007; Wang et al., 2006). Therefore, newly synthesized histone proteins (that is, from the polyadenylated histone mRNAs) may help maintain proper chromatin structure subsequent to DNA repair. In this way, the accumulation of polyadenylated histone transcripts may be an additional way in which p53 accumulation helps maintain genomic integrity. Future studies, which specifically address the role of these transcripts in the protective effects of p53 accumulation before DNA damage and the expression of 
these transcripts in primary tumor samples, will further clarify their physiological importance.

\section{Materials and Methods}

\section{Cell culture, transfection and western blot analysis}

HCT116 cells were grown in McCoy's medium containing 10\% fetal bovine serum (FBS) and $1 \times$ penicillin/streptomycin and treated with Nutlin-3a (Sigma, Munich, Germany) as indicated. Transfection of siRNAs (listed in Supp Table III.S1) was performed using Lipofectamine RNAiMAX (Invitrogen, Heidelberg, Germany) according to the manufacturer's instructions. Western blot analysis was performed using the antibodies listed in Supp Table III.S2.

\section{Co-immunoprecipitation and GST pull-down analyses}

Co-immunoprecipitation analysis was performed as previously described (Shin et al., 2007) using the indicated antibodies as listed in Supp Table III.S2. GST pull-down analyses were performed as previously described (Johnsen et al., 2002).

\section{ChIP and gene expression analysis}

ChIP analyses using the antibodies and dilutions listed in Supp Table III.S2 were performed as previously described (Pirngruber et al., 2009b). RNA was harvested with Qiazol (Qiagen, Hilden, Germany) according to the manufacturer's instructions and reverse transcribed using either oligo-dT or random nonamer primers as indicated in the respective figure legends. DNA samples (ChIP or cDNA) were analyzed by SYBR Green-based real-time PCR using a relative standard curve and a PCR buffer $(75 \mathrm{mM}$ Tris- $\mathrm{HCl}$ (pH 8.8), $20 \mathrm{mM}\left(\mathrm{NH}_{4}\right)_{2} \mathrm{SO}_{4}, 0.01 \%$ Tween-20) containing $3 \mathrm{mM} \mathrm{MgCl} 2,200$ $\mu \mathrm{M}$ dNTPs, $0.5 \mathrm{U}$ per reaction Taq DNA polymerase (PrimeTech, Minsk, Belarus), 0.25\% Triton X-100, 1:80,000 SYBR Green I (Roche, Mannheim, Germany), 300 mM trehalose and 300 nM primers. Primers used are listed in Supp Tables III.S3 and III.S4. Experimental background was determined by performing a control ChIP with a nonspecific IgG. The average of the background signals for all primer pairs were similar and are graphically represented by the dotted line. All quantitative reverse transcriptase PCRs were normalized to RPLPO before additional normalization and statistical analyses. 


\section{Immunofluorescence microscopy}

Indirect immunofluorescence stainings were performed as previously described (Lemm et al., 2006) using the antibodies as listed in Supp Table III.S2. Fluorescent-labeled samples were visualized under identical conditions for control and Nutlin-3a-treated cells using a Zeiss LSM Meta 510 confocal microscope (Oberkochen, Germany).

\section{Flow cytometry}

Cells were prepared for propidium iodide-based flow cytometric cell-cycle analysis as described (Kranz and Dobbelstein, 2006) and analyzed using a Guava EasyCyte plus (Guava Technologies, Hayward, CA), and data were analyzed and graphically displayed using ModFit (Verity Software House, Topsham, ME). 5-Bromodeoxyuridine analyses were performed essentially as described (Kranz et al., 2008) using the antibodies listed in Supp Table III.S2. Annexin V apoptosis assays were performed using the Guava Nexin Reagent (Guava Technologies) according to the manufacturer's instructions. 


\subsubsection{Supplementary Material}

\section{Supplementary Tables}

Supp Table III.S1: siRNAs utilized for knockdown studies in a 5' to 3' orientation.

\begin{tabular}{|c|c|c|c|}
\hline Target Gene & siRNA Target Sequence & Source & Reference \\
\hline CDK9 & TAGGGACATGAAGGCTGCTAA & $\begin{array}{l}\text { Qiagen } \\
\text { (validated) }\end{array}$ & (Pirngruber et al., 2009b) \\
\hline Cyclin E1 & GCTTCGGCCTTGTATCATTTT & $\begin{array}{l}\text { Ambion } \\
\text { (validated) }\end{array}$ & - \\
\hline Control (Qiagen) & - & Qiagen & - \\
\hline $\begin{array}{l}\text { Control (ABI) } \\
\text { Neg. Cont \#2 }\end{array}$ & 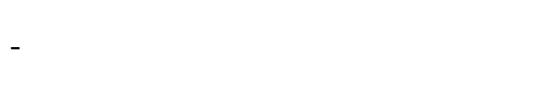 & $\mathrm{ABI}$ & - \\
\hline NPAT \#1 & GGCACAUCUUCAAACAAUA & Qiagen & (Barcaroli et al., 2006a) \\
\hline NPAT \#2 & GGUUUGCUGGCAGUCAGAG & Qiagen & (Gao et al., 2003) \\
\hline
\end{tabular}

Supp Table III.S2: Antibodies used for ChIP, IP, western blot analyses and Immunofluorescence and the respective dilutions.

\begin{tabular}{|c|c|c|c|c|c|c|c|}
\hline $\begin{array}{l}\text { Target } \\
\text { Protein }\end{array}$ & Clone & Cat. Nr. & ChIP & IP & WB & IF & Source/Reference \\
\hline BrdU & - & ab8955 & - & - & - & - & Abcam \\
\hline CDK9 & $\mathrm{C}-20$ & sc-484 & $1 \mu \mathrm{g}$ & $3 \mu \mathrm{g}$ & $1: 1000$ & - & Santa Cruz \\
\hline CDK9 & D-7 & sc-13130 & - & - & $1: 1000$ & - & Santa Cruz \\
\hline Coilin & $5 \mathrm{P} 10$ & - & - & - & - & $1: 500$ & (Almeida et al., 1998) \\
\hline Cyclin T1 & $\mathrm{H}-245$ & sc-10750 & - & - & $1: 1000$ & - & Santa Cruz \\
\hline $\mathrm{E} 2 \mathrm{~F} 1$ & $\mathrm{C}-20$ & sc-193 & $2 \mu \mathrm{g}$ & - & - & - & Santa Cruz \\
\hline E2F4 & A-20 & sc-1082 & $2 \mu \mathrm{g}$ & - & - & - & Santa Cruz \\
\hline $\begin{array}{l}\text { Histone } \\
\mathrm{H} 2 \mathrm{~B}\end{array}$ & - & $07-731$ & - & - & $1: 3000$ & - & Upstate \\
\hline Hsc70 & B6 & sc-7298 & - & - & $1: 25,000$ & - & Santa Cruz \\
\hline $\begin{array}{l}\text { IgG (Non- } \\
\text { specific) }\end{array}$ & - & ab46540 & $1 \mu g$ & $3 \mu \mathrm{g}$ & - & - & Abcam \\
\hline NPAT & - & - & $2 \mu 1$ & $5 \mu 1$ & $1: 1000$ & $1: 5000$ & (Zhao et al., 2000) \\
\hline P130 & $\mathrm{C}-20$ & sc-317 & $2 \mu \mathrm{g}$ & - & - & - & Santa Cruz \\
\hline P21 & EA10 & OP64 & - & - & $1: 500$ & - & Calbiochem \\
\hline P53 & DO-1 & sc-126 & - & - & $1: 1000$ & - & Santa Cruz \\
\hline PAF1 & - & $\begin{array}{l}\mathrm{pAb}-015- \\
050\end{array}$ & $1 \mu \mathrm{g}$ & - & - & - & $\begin{array}{l}\text { Diagenode } \\
\text { (Zhu } \text { et al., 2005a) }\end{array}$ \\
\hline $\mathrm{RB}$ & - & 554136 & - & - & $1: 250$ & - & BD Biosciences \\
\hline $\begin{array}{l}\text { RNAPII } \\
\text { (N-Term) }\end{array}$ & $\mathrm{N}-20$ & sc- 899 & - & - & - & - & Santa Cruz \\
\hline $\begin{array}{l}\text { Donkey } \\
\text { Anti Mouse } \\
\text { IgG-HRP } \\
\text { Donkey }\end{array}$ & - & $\begin{array}{l}715-036- \\
150\end{array}$ & - & - & $1: 10,000$ & - & $\begin{array}{l}\text { Jackson } \\
\text { ImmunoResearch }\end{array}$ \\
\hline $\begin{array}{l}\text { Anti } \\
\text { Rabbit IgG- } \\
\text { HRP }\end{array}$ & - & $\begin{array}{l}711-036- \\
152\end{array}$ & - & - & $1: 10,000$ & - & $\begin{array}{l}\text { Jackson } \\
\text { ImmunoResearch }\end{array}$ \\
\hline $\begin{array}{l}\text { Donkey } \\
\text { Anti Rabbit }\end{array}$ & - & $\begin{array}{l}711-165- \\
152\end{array}$ & - & - & - & $1: 200$ & $\begin{array}{l}\text { Jackson } \\
\text { ImmunoResearch }\end{array}$ \\
\hline
\end{tabular}


IgG-Cy3

Goat Anti

Mouse IgG-

A21202

$1: 500$

Molecular Probes

Alexa 488

Supp Table III.S3: Primers utilized for chromatin immunoprecipitation analyses in 5' to 3' orientation. Primers not obtained from other previous studies were designed using the PrimerBlast program (http://www.ncbi.nlm.nih.gov/tools/primer-blast/).

\begin{tabular}{lll} 
ChIP Primers & & Source \\
\hline HIST1H2BD TSS F & ACCTCATTTGAATACCGCATCT & (Pirngruber et al., 2009b) \\
HIST1H2BD TSS R & TGGTAGGTTCAGGCATCGTAG & (Pirngruber et al., 2009b) \\
HIST1H2BD +0.5kb F & TCTTTTAAGAGCCACGCATGT & (Pirngruber et al., 2009b) \\
HIST1H2BD +0.5kb R & CGTAACATTAAAGATTGGTACTGTGG & (Pirngruber et al., 2009b) \\
HIST1H2AC TSS F & AAAAGCGGCCATGTTTTACA & (Pirngruber et al., 2009b) \\
HIST1H2AC TSS R & AAAAATCACCAAAACCAGCG & (Pirngruber et al., 2009b) \\
HIST1H2AC +0.5kb F & CCCGGAAACGCTATCAAAC & This manuscript. \\
HIST1H2AC +0.5kb R & TAGTAACAGCGCTCCCCAAA & This manuscript. \\
NPAT E2F-BS F & CAGGAGCTGTCGCTGTGTTTG & (Gao et al., 2003) \\
NPAT E2F-BS R & GGCTACGTCCGAGGGTAACAA & (Gao et al., 2003) \\
P107 E2F-BS F & AGGCAGACGGTGGATGACAACAC & (Takahashi et al., 2000) \\
P107 E2F-BS R & TCAGCGTGGGGCTTGTCCTCGAA & (Takahashi et al., 2000) \\
TFF1 +6kb F & CAGGCTTCTCCCTTGATGAAT & This manuscript. \\
TFF1 +6kb R & ACACCCACCTTCCACAACAC & This manuscript.
\end{tabular}

Supp Table III.S4: Primers utilized for gene expression analyses in 5' to 3' orientation. Primers not obtained from other previous studies were designed using the PrimerBlast program (http://www.ncbi.nlm.nih.gov/tools/primer-blast/).

RT-PCR Primers

Cyclin E1 F

Cyclin E1 R

HIST1H2BD Total F

HIST1H2BD Total R

HIST1H2BD PolyA F

HIST1H2BD PolyA R

HIST1H2AC Total F

HIST1H2AC Total R

HIST1H2AC PolyA F

HIST1H2AC PolyA R

NPAT F

NPAT R

MDM2 F

MDM2 R

p21 F
Source

This manuscript.

This manuscript.

(Pirngruber et al., 2009b)

(Pirngruber et al., 2009b)

(Pirngruber et al., 2009b)

(Pirngruber et al., 2009b)

(Pirngruber et al., 2009b)

(Pirngruber et al., 2009b)

(Pirngruber et al., 2009b)

(Pirngruber et al., 2009b)

This manuscript.

This manuscript.

(Hobom and Dobbelstein, 2004)

(Hobom and Dobbelstein, 2004)

(Gomes et al., 2006) 
p21 R

GCTGAGAGGGTACTGAAGGGAAA

(Gomes et al., 2006)

RPLP0 F

\section{Supplementary Figures}

A
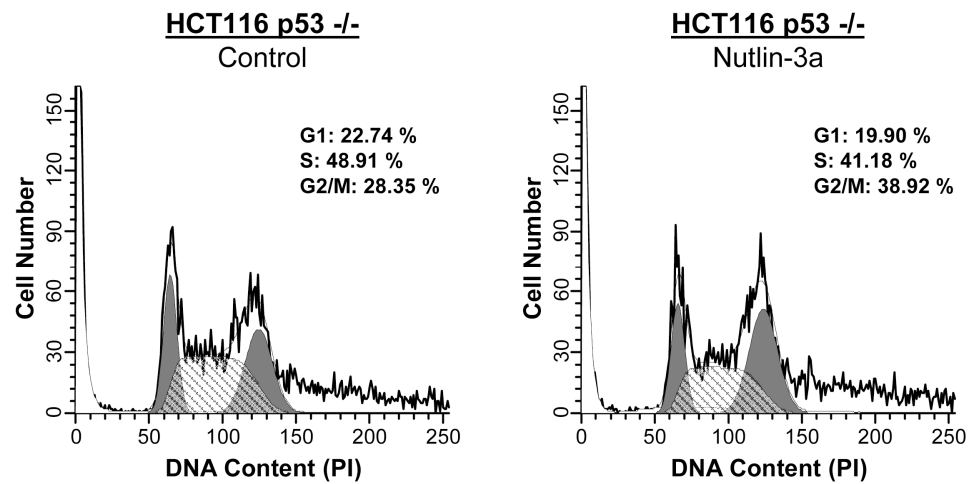

B
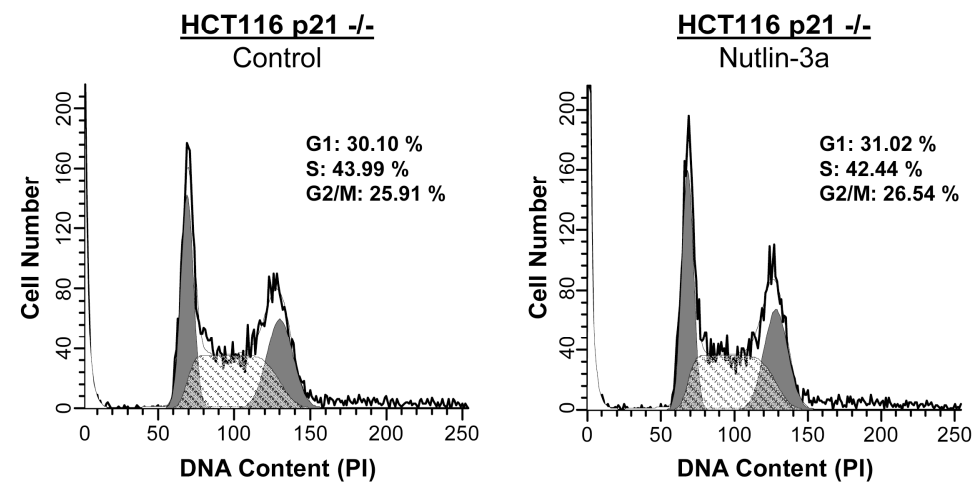

Supp Fig III.S1: Nutlin-3a treatment does not affect cell-cycle progression in the absence of p53 or p21. HCT116 cells which were null for either p53 (A) or p21 (B) were treated with $8 \mu \mathrm{M}$ Nutlin-3a for 24 $\mathrm{h}$ and analyzed by flow cytometry for cell cycle analyses as in Fig III.1A. Note that no significant change in any of the cell-cycle phases was observed. 

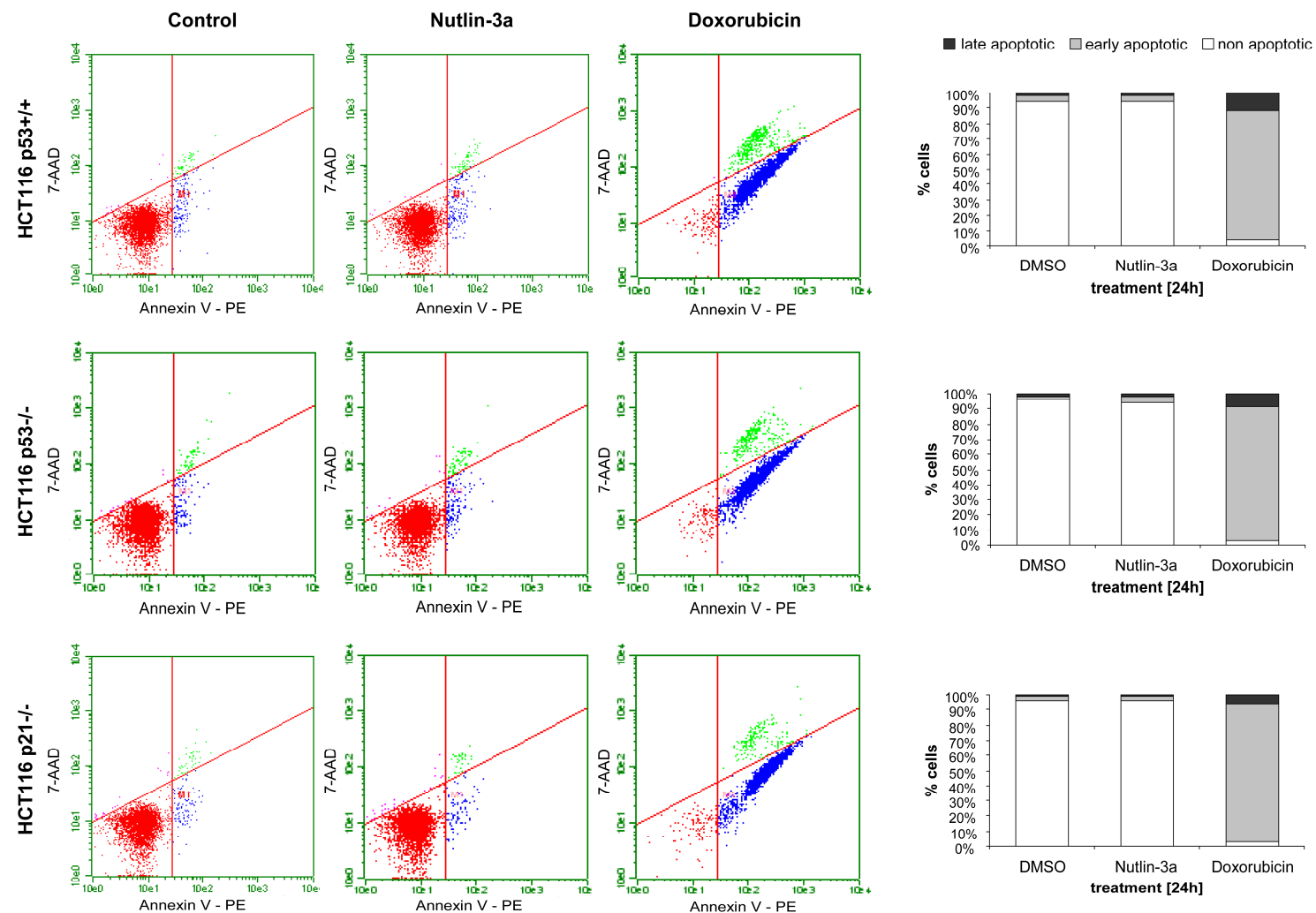

Supp Fig III.S2: Nutlin-3a treatment does not significantly induce apoptosis in HCT116 cells irrespective of the p53 and p21 status. Apoptosis in untreated wild-type (p53+/+), p53 null (p53-/-) or p21 null (p21-/-) HCT116 cells was compared to treatments with Nutlin-3a ( $8 \mu \mathrm{M}, 24 \mathrm{~h})$ or Doxorubicin (as a positive control, $5 \mu \mathrm{M}, 24 \mathrm{~h}$ ) and analyzed using the Guava Nexin Kit (Guava Technologies) according to the manufacturer's protocol. Cells in the lower left gated quadrant (Annexin V negative/7AAD negative) reflect non-apoptotic cells, cells in the lower right quadrant (Annexin V positive/7-AAD negative) reflect early apoptotic cells, and cells in the upper right quadrant (Annexin V positive/7-AAD positive) reflect late apoptotic cells. The fractions of gated cells were quantitated and are graphically represented for each cell line on the right.

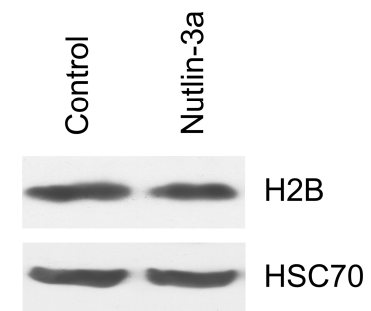

Supp Fig III.S3: Total H2B protein levels do not change following Nutlin-3a treatment. The expression of $\mathrm{H} 2 \mathrm{~B}$ protein levels was investigated in protein samples from HCT116 cells $24 \mathrm{~h}$ after treatment with DMSO (control) or Nutlin-3a $(8 \mu \mathrm{M})$ using an antibody that detects $\mathrm{H} 2 \mathrm{~B}$. HSC70 is shown as a loading control. 


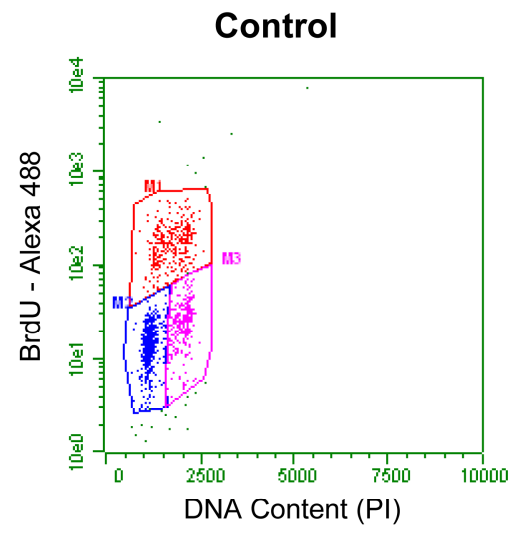

$4 \mathrm{~h}$
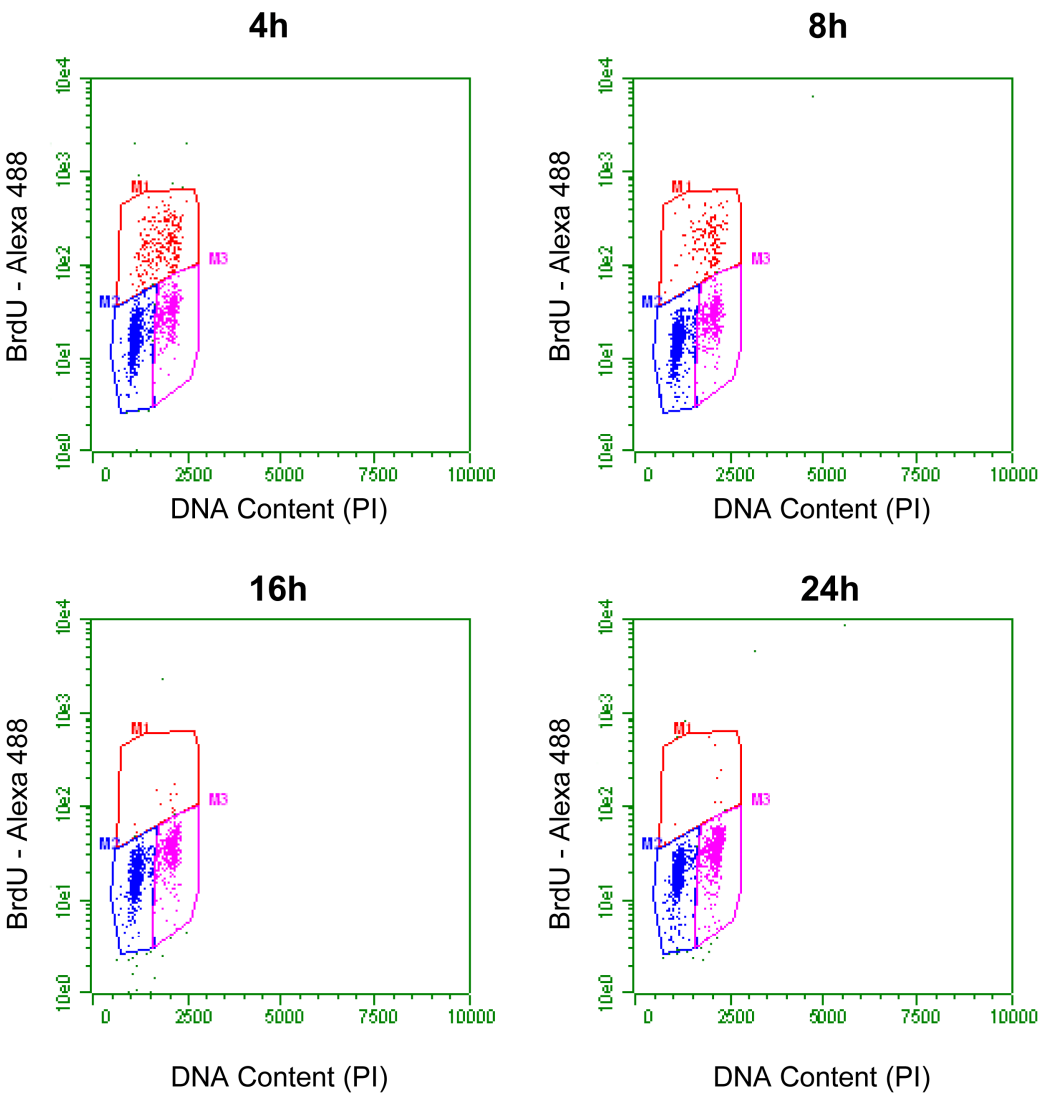

Supp Fig III.S4: Nutlin-3a time-dependently decreases the fraction of HCT116 cells in S phase. The fraction of cells in S phase following Nutlin-3a treatment $(8 \mu \mathrm{M})$ was investigated by combined BrdU/PI staining and analyzed by flow cytometry. The fraction of $\mathrm{S}$ phase cells (upper gated fraction) was compared to the total cell counts and is graphically represented in Fig III.3D. 
A

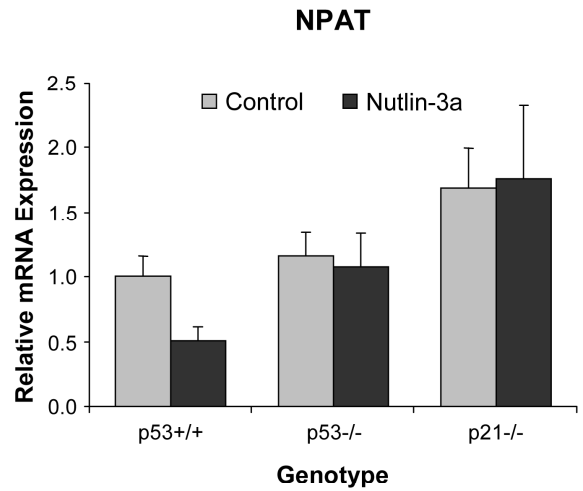

B
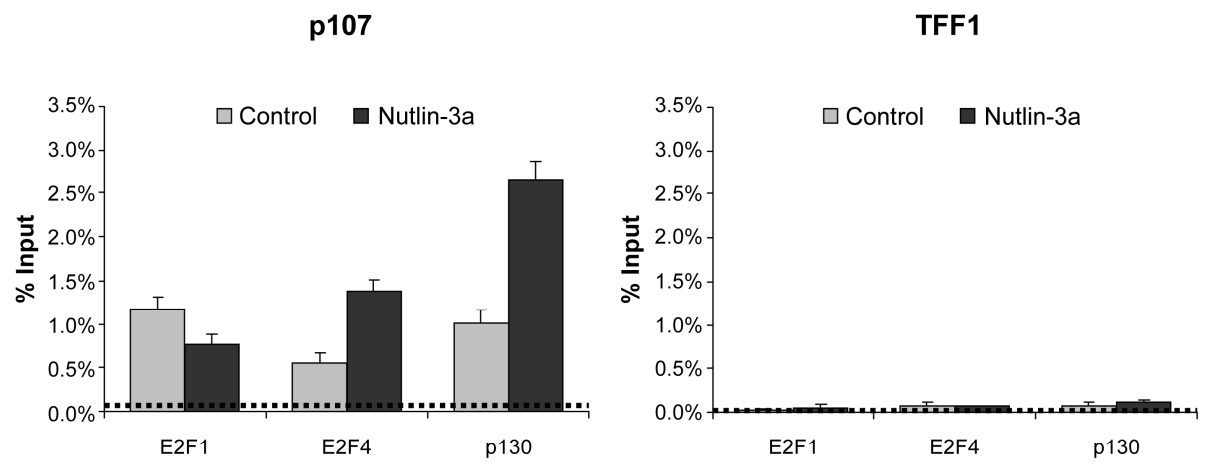

C

HCT116 cells

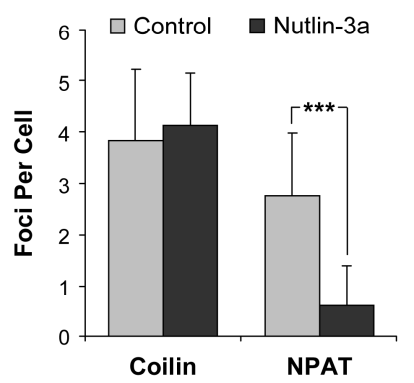

NPAT

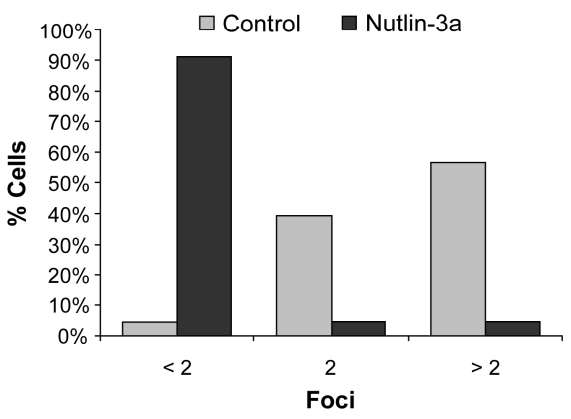

Supp Fig III.S5: (A) NPAT downregulation depends upon an intact p53-p21 axis. The expression of NPAT mRNA was analyzed by random-primed qRT-PCR as in Fig III.3B using the same samples shown in Fig III.1C-E. Gene expression levels were normalized and expressed as in Fig III.1; mean values + s.d., $n=3$. (B) The profile of E2F1, E2F4 and p130 binding to the E2F target gene p107 following Nutlin-3a treatment. The same samples as in Fig III.3F were analyzed by ChIP for the binding of E2F1, E2F4 and p130 to the 107 (left panel) and TFF1 (control locus, right panel) genes. qChIP values were normalized as in Fig III.2; mean values + s.d., $n=3$. The experimental background is shown as a dotted line. (C) P53-induced cell-cycle arrest does not affect Cajal body integrity. The effects of Nutlin-3a treatment on NPAT-positive histone locus body and Cajal body structures were quantitated by counting the number of NPAT-positive or Coilin-positive foci in cells from at least three random fields for each treatment which were photographed by confocal laser microscopy as shown in Fig III.3G. The average number of foci only significantly differed for NPAT (left panel, $n=23$ ). These effects were even more noticeable when the number of cells with less than 2, exactly two, or more than 2 NPAT foci were counted. Note that while most untreated cells contain at least two NPAT foci, more than $90 \%$ of Nutlin-3a treated cells have 0-1 NPAT foci/cell. 
A

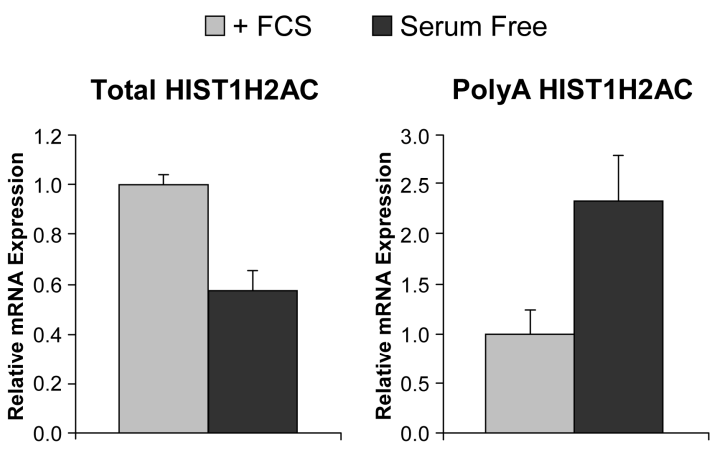

B

$\square$ Control siRNA $\square$ Cyclin E1 siRNA

Total HIST1H2AC

PolyA HIST1H2AC
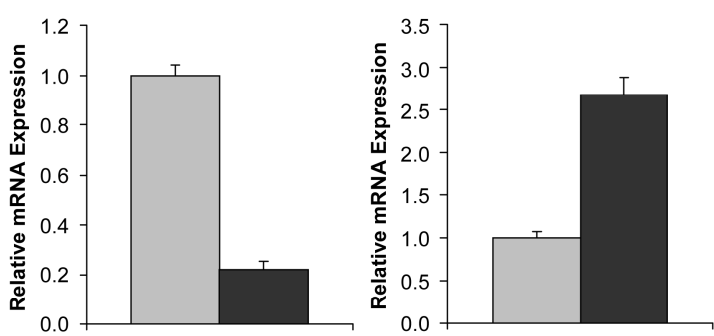

C $\square$ Control

Hydroxyurea 48h

Total HIST1H2AC

PolyA HIST1H2AC
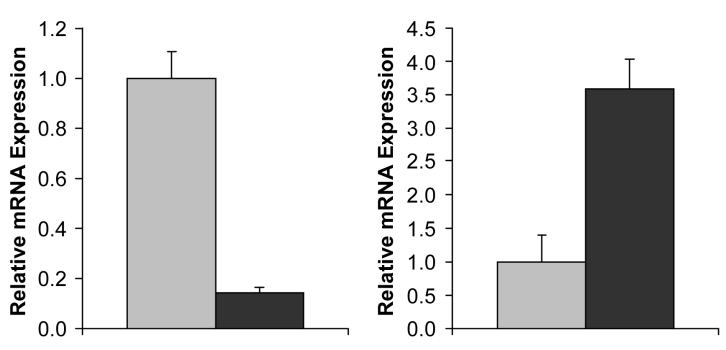

Supp Fig III.S6: Accumulation of polyadenylated replication-dependent histone mRNAs is induced following a $\mathbf{G}_{\mathbf{1}} / \mathbf{G}_{\mathbf{0}}$ cell-cycle arrest. (A) Serum starvation down-regulates total HIST1H2AC mRNA levels but increases mRNA polyadenylation. HIST1H2AC gene expression levels from the samples analyzed in Fig III.4B were normalized and expressed as in Fig III.1; mean values + s.d., $n=2$. (B) Cyclin E1 knockdown similarly controls the expression of total and polyadenylated HIST1H2BD (Fig III.4E) and HIST1H2AC mRNA (B) as assessed in Fig III.1 using the same RNA samples as in Fig III.4C,E. Gene expression levels were normalized and expressed as in Fig III.1; mean values + s.d., $n=2$. (C) The expression of total and polyadenylated HIST1H2AC was analyzed as in Fig III.1D with the same samples shown in Fig III.4F after treatment of HCT116 cells with $2 \mathrm{mM}$ hydroxyurea for $48 \mathrm{~h}$. Gene expression levels were normalized and expressed as in Fig III.1; mean values + s.d., $n=2$. 
A

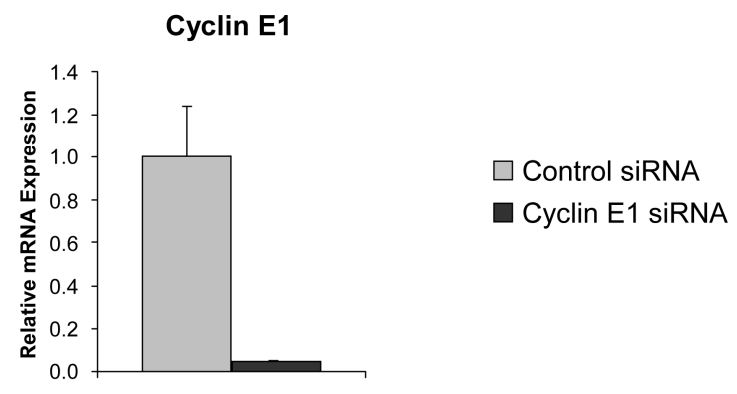

B

$\square$ Control siRNA $\square$ Cyclin E1 siRNA

Total HIST1H2BD

PolyA HIST1H2BD
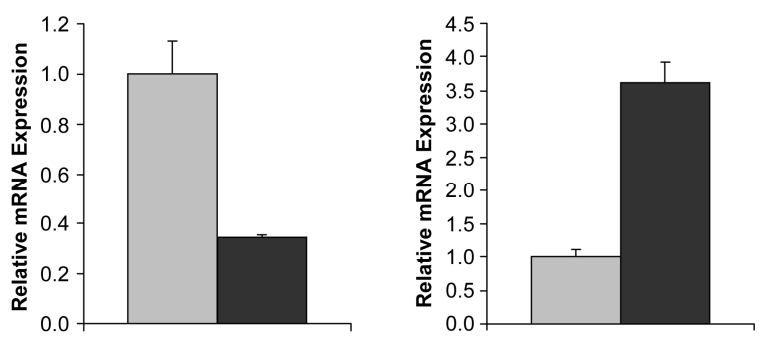

C

Total HIST1H2AC

PolyA HIST1H2AC
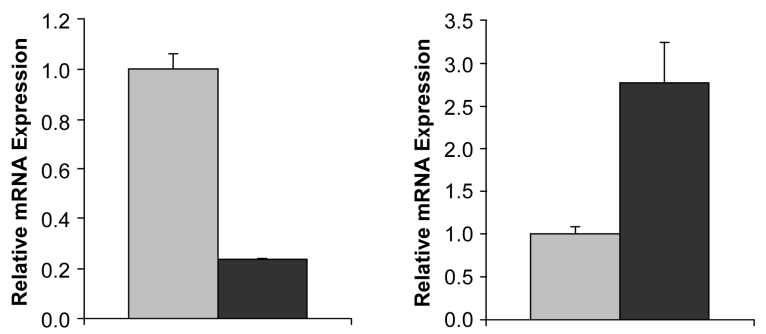

Supp Fig III.S7: The effect of Cyclin E1 on replication-dependent histone gene expression and premRNA 3' end processing is independent of p53 status. The Cyclin E1 siRNA utilized in Fig III.4C-E and Supp Fig III.S6B was transfected into p53 null (p53-/-) HCT116 cells and analyzed for the expression of Cyclin E1 (A), total and polyadenylated HIST1H2BD (B) as well as total and polyadenylated HIST1H2AC (C) mRNAs. Gene expression levels were normalized and expressed as in Fig III.1; mean values + s.d., $n=2$. 
A

$\square$ Control siRNA $\square$ NPAT siRNA \#2

Total HIST1H2BD

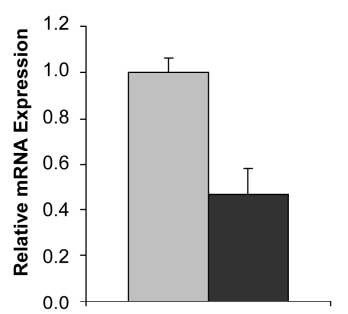

B Total HIST1H2AC

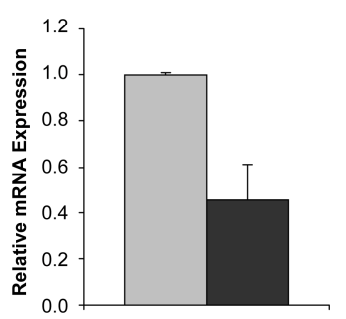

C

$\square$ Control siRNA $\square$ NPAT siRNA \#2

NPAT

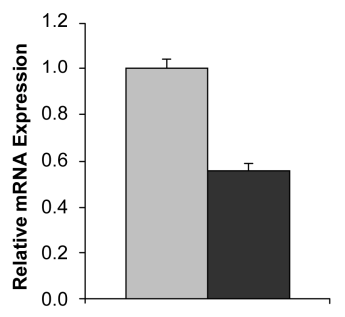

E

$\square$ Control siRNA $\square$ CDK9 siRNA
PolyA HIST1H2BD

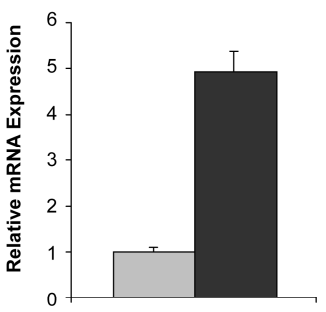

PolyA HIST1H2AC

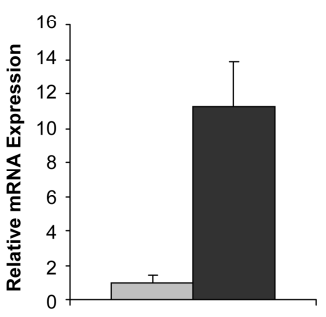

D

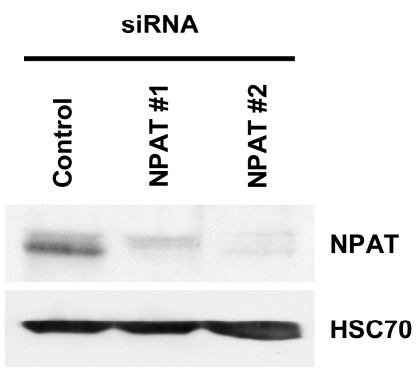

PolyA HIST1H2BD

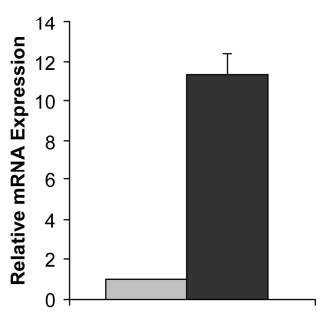

Supp Fig III.S8: NPAT expression is essential for optimal levels of replication-dependent gene transcription and proper mRNA 3' end processing. A second siRNA targeting NPAT (siRNA \#2) was used to perform similar analyses as in Fig III.5A-C. (A,B) The effects of this siRNA on the expression of total and polyadenylated HIST1H2BD (A) and HIST1H2AC (B) mRNAs was assessed in HCT116 cells $72 \mathrm{~h}$ after transfection with control or NPAT siRNAs as in Fig III.5. (C) The effectiveness of NPAT siRNA \#2 was assessed by random-primed qRT-PCR using the same RNA samples as in (A,B). Gene expression levels were normalized and expressed as in Fig III.1; mean values + s.d., $n=2$. (D) The knockdown of NPAT protein levels following transfection with either NPAT siRNA were verified by western blot analysis in cell lysates prepared in parallel to the mRNA samples shown in Fig III.5A-C and Supp Fig III.S8A-C. (E) The effects of CDK9 knockdown on replication-dependent histone mRNA 3' end processing were confirmed in HCT116 cells $72 \mathrm{~h}$ after transfection with control or CDK9 siRNAs. Gene expression levels were normalized and expressed as in Fig III.1; mean values + s.d., $n=2$. 
A

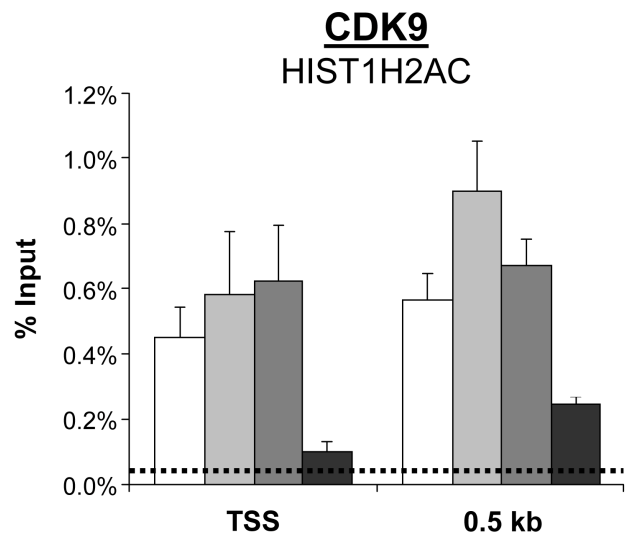

$\square$ mock

$\square$ Control siRNA (Qiagen)

$\square$ Control siRNA (ABI)

- NPAT SIRNA \#1

B

PAF1

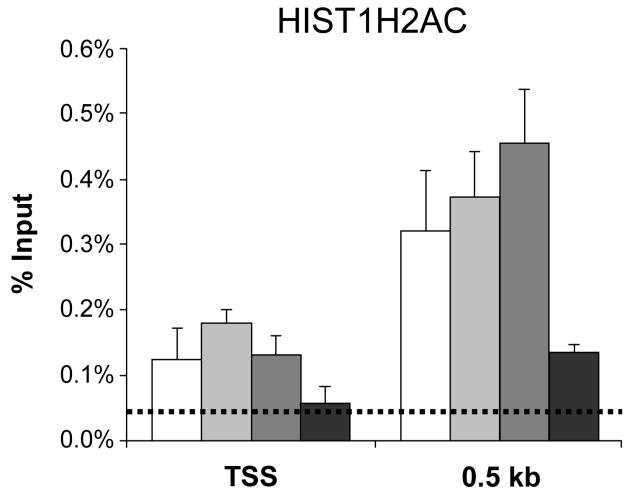

C

NPAT

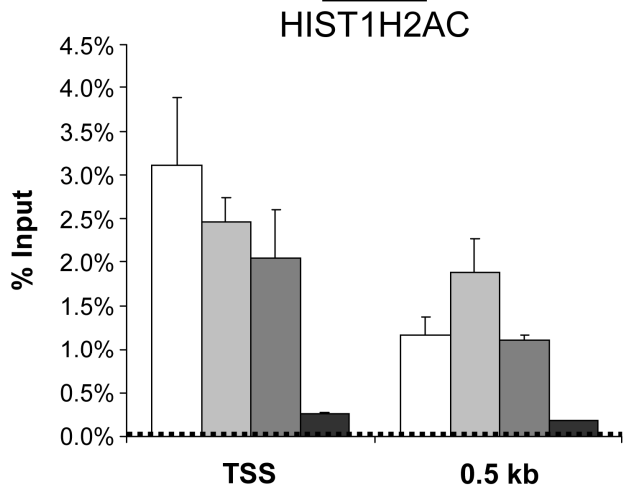

Supp Fig III.S9: Decreased CDK9, PAF1 and NPAT recruitment to replication-dependent histone genes is a specific effect of NPAT knockdown and not due to non-specific effects of siRNA transfection. Chromatin immunoprecipitation experiments were performed as in Fig III.2 and III.6 using HCT116 cells that were mock transfected (i.e., water only), transfected with two different control siRNAs, or a specific siRNA against NPAT for $72 \mathrm{~h}$. The recruitment of CDK9 (A), PAF1 (B) and NPAT (C) was investigated at both the transcriptional start site (TSS) and 3' end cleavage site $(0.5 \mathrm{~kb})$ of the HISTIH2AC gene. Only minor differences between the three control samples were observed. In contrast, substantial differences in the recruitment of all three factors between the control and NPAT knockdown cells was observed particularly at $0.5 \mathrm{~kb}$. 


\section{General Discussion}

\subsection{Chromatin structure, histone modifications and the CTD of RNAPII}

Chromatin structure, histone modifications and the phosphorylation status of the CTD of RNAPII have been associated with transcriptional regulation (Berger, 2007; Buratowski, 2003; Egloff and Murphy, 2008). Our concern was to get insights into the function of CTD phosphorylation, especially Ser2 phosphorylation by CDK9, in influencing posttranslational histone modifications. Whereas quite a lot was already known in yeast, this aspect still needed to be investigated in humans. Thus, our main focus was to explore the connection between CTD phosphorylation and histone $\mathrm{H} 2 \mathrm{~B}$ monoubiquitination which led us to a model in which CDK9 regulates not only H2Bub1 but a hierarchy of histone modifications by CTD-dependent and -independent mechanisms. Hence, we discovered new and important similarities and distinctions between the functions of P-TEFb (CDK9/Cyclin T1) in yeast and humans.

\subsubsection{A CTD-independent way for monoubiquitination of human histone H2B?}

As already mentioned in section 1.2.1.1 on page 11f., monoubiquitination of histone H2B is coupled to ongoing transcription (Osley, 2006; Shilatifard, 2006). For investigation of the distribution of H2Bub1 on active human genes Minsky et al. generated an anti-H2Bub1 monoclonal antibody (Minsky et al., 2008). Genome wide analysis of H2Bub1 distribution revealed a preferred association of monoubiquitinated histone $\mathrm{H} 2 \mathrm{~B}$ with the transcribed region of highly expressed genes, more precisely, H2Bub1 levels starting near the transcriptional start site gradually increased into the transcribed region. To obtain these data a total of 16,101 genes were used for a ChIPmicroarray. Interestingly, this distribution pattern of H2Bub1 on active genes resembles that of Ser2-phosphorylated RNAPII on the activated p21 gene (Gomes et al., 2006). In this study twenty different positions on the active $p 21$ gene were investigated by ChIP analysis using an antibody specific for Ser2-phosphorylated RNAPII. This correlation led us to the hypothesis that H2Bub1 could be dependent upon P-Ser2 CTD and thus upon the activity of CDK9, which phosphorylates Ser2 residues of the CTD in humans (Marshall et al., 1996). Although P-Ser2 and H2Bub1 are disconnected in yeast and instead P-Ser5 of the CTD seems to play a role since inactivation of Kin28p, the yeast 
Ser5 kinase, leads to decreased H2Bub1 levels, a link between H2Bub1 and CTD phosphorylation in humans has not been investigated so far (Xiao et al., 2005).

Our results indeed show that CDK9-dependent phosphorylation of RNAPII CTD at Ser2 is important for monoubiquitination of histone H2B. Knockdown of CDK9 as well as deletion or mutation of the RNAPII CTD led to decreased H2Bub1 protein expression whereas overexpression of CDK9 resulted in an increased level of H2Bub1 (illustrated in Fig I.2B-D on page 38). Our model suggests that P-Ser2 serves as a platform for the binding of the PAF complex which stimulates the activity of RNF20/40 which then monoubiquitinates histone H2B. This model has already been established for yeast with the exception of P-Ser5 being the CTD modification that plays a role in recruiting the yeast PAF complex to the elongating RNAPII (Wood et al., 2003b; Xiao et al., 2005).

While inhibition and knockdown of CDK9 led to a strong decrease to almost non-detectable H2Bub1 protein levels, either deletion of the whole CTD or mutation of the Ser2 residue of the CTD to alanine that can not be phosphorylated resulted in only a moderate loss of H2Bub1 (illustrated in Fig I.2B,D on page 38). This suggests that $\mathrm{H} 2 \mathrm{Bub} 1$ is regulated primarily through CDK9-dependent CTD phosphorylation but could in addition also be regulated in a different way.

In yeast, the E2 ubiquitin ligase implemented in monoubiquitination of histone H2B, Rad6p, is phosphorylated on Ser120 by Bur1p, a yeast homolog of human CDK9, and this is critical for fully catalyzing monoubiquitination of histone $\mathrm{H} 2 \mathrm{~B}$ in vivo (Wood et al., 2005). Interestingly, this effect appears to be independent of CTD phosphorylation since the phosphorylation of CTD at Ser2 and Ser5 is unaffected in Burlp mutant strains (Keogh et al., 2003). The human homolog of Rad6p was also shown to be phosphorylated at Ser120 by cyclin-dependent kinases in vivo (Sarcevic et al., 2002). Since CDK9 likely has various target proteins and is the homolog of Bur1p it could be possible that it can also phosphorylate the human Rad6p homolog UBE2A. Unpublished results of our group show that CDK9 can indeed phosphorylate UBE2A and that this can occur in the absence of the CTD.

Additionally, our ChIP data show a clear decrease of RNF20, RNF40 and PAF recruitment to the $H I S T 1 H 2 B D$ gene upon knockdown of CDK9 (illustrated in Fig I.4C on page 42). However, there is still a detectable level of these proteins on the gene. Why then is H2Bub1 still essentially undetectable on this gene although there are detectable levels of RNF20, RNF40 and PAF? Maybe this is because the function of UBE2A is 
impaired in the absence of phosphorylation by CDK9. Having this second "pathway" the strong decrease in $\mathrm{H} 2 \mathrm{Bub} 1$ on the HIST1H2BD gene despite the moderate decrease in binding of RNF20, RNF40 and PAF to this gene after CDK9 knockdown could be explained.

Another support for a second way of monoubiquitination of H2B by UBE2A is the fact that this protein plays an important role in DNA damage response (Game and Chernikova, 2009). For DNA repair the chromatin cohesion has to be altered meaning that nucleosomes have to be removed and later on replaced by histone chaperones. H2Bub1 has been shown to be essential for the function of the FACT chaperone in yeast which removes an $\mathrm{H} 2 \mathrm{~A} / \mathrm{H} 2 \mathrm{~B}$ dimer from nucleosomes (Laribee et al., 2007). Interestingly, FACT was shown to play a role in exchanging the variant histone $\mathrm{H} 2 \mathrm{AX}$ with the canonical histone $\mathrm{H} 2 \mathrm{~A}$ by facilitating both incorporation or dissociation of H2AX from the nucleosome (Heo et al., 2008). Phosphorylation of H2AX which is implicated in the cellular response to DNA damage facilitates FACT-mediated dissociation of $\mathrm{H} 2 \mathrm{AX}$ via nucleosomal destabilization whereas ADP-ribosylation of the FACT subunit SUPT16H results in release of FACT from the nucleosome and thus stabilization. These results suggest a critical role for FACT during DNA repair processes. It is tempting to speculate that a histone octamer containing phosphorylated $\mathrm{H} 2 \mathrm{AX}$ and H2Bub1 might serve as an even more efficient substrate for FACT. In support of a role for $\mathrm{H} 2 \mathrm{Bub} 1$ in controlling the DNA damage response, collaborative work from our group together with the group of Moshe Oren showed an effect of the H2B E3 ubiquitin ligase RNF20 on apoptosis induced by 5-Fluorouracil (5-FU), a chemotherapeutic agent that incorporates into DNA and leads to DNA damage (Shema et al., 2008). Depletion of RNF20 resulted in a decrease in apoptotic p53-positive cells and is thus thought to be required for maintaining maximal p53 functionality. Moreover, RNF20 also sustains the expression of the tumor protein p53 binding protein 1 (TP53BP1) which is an important component of the cellular response to double-strand DNA breaks. Rad6p is known to play an important role in DNA damage response as well (Game et al., 2009). However, a possible implementation of H2Bub1 in DNA repair mechanisms has not been demonstrated. Indeed, Rad6p not only monoubiquitinates histone $\mathrm{H} 2 \mathrm{~B}$ but also the proliferating cell nuclear antigen (PCNA) protein and this pathway is rather known to play a function in DNA damage response. However, altogether these studies suggest a possible role of $\mathrm{H} 2 \mathrm{Bub} 1$ in altering the chromatin structure upon DNA damage. 


\subsubsection{The role of H2Bub1 in chromatin assembly and disassembly}

As already mentioned in section 3.1 on page 95 , monoubiquitination of histone $\mathrm{H} 2 \mathrm{~B}$ is suggested to play an important role in altering the cohesion of the chromatin and was thereby thought to be implemented in opening and loosening nucleosomes (Laribee et al., 2007; Pavri et al., 2006). More detailed, H2Bub1 was shown to interact with Spt16p in yeast, a subunit of the histone $\mathrm{H} 2 \mathrm{~A} / \mathrm{H} 2 \mathrm{~B}$ chaperone FACT, and was thought to control nucleosome eviction in front of elongating RNAPII (Pavri et al., 2006). Controversely, in 2003 it was already suggested that FACT could play a role in reversing nucleosome disruption that occurs during RNAPII elongation and could thus prevent internal initiation from cryptic promoters which was observed if Spt16p was lost (Mason and Struhl, 2003). In addition, previous studies in yeast revealed that H2B monoubiquitination increases as cells enter prophase and decreases after metaphase/anaphase transition (Mueller et al., 1985; Sarcevic et al., 2002). This leads to the suggestion that after DNA replication $\mathrm{H} 2 \mathrm{Bub} 1$ could play a role in reassembling nucleosomes so that the DNA can be properly condensed before mitosis takes place. With regard to our results, this would mean that a decrease in H2Bub1 would lead to improper condensation of chromatin prior to mitosis and thus defects in cell cycle distribution. However, neither knockdown of CDK9 which we showed is important for monoubiquitination of $\mathrm{H} 2 \mathrm{~B}$ nor knockdown of RNF20/40 had an effect on cell cycle distribution (data not shown). If $\mathrm{H} 2 \mathrm{Bub} 1$ functions more in nucleosome reassembly than in nucleosome removal thus still needs to be investigated.

Furthermore, recent studies left the question unanswered if H2Bub1 directly stimulates transcription or in contrast if transcription facilitates H2Bub1. In this regard, Kim and co-workers recently showed that $\mathrm{H} 2 \mathrm{Bub} 1$ alone is unable to directly affect transcription through chromatin but that ongoing transcription is required for efficient H2B monoubiquitination (Kim et al., 2009). This provides an explanation why H2Bub1-dependent $\mathrm{H} 3 \mathrm{~K} 4 \mathrm{me} 3$ is a mark for recent transcription ( $\mathrm{Ng}$ et al., 2003). Interestingly, in contrast to Pavri et al., they claim that they could not detect a direct function for H2Bub1 in activating FACT in vitro (Pavri et al., 2006). However, this could in part be due to the usage of the nucleosome assembly protein 1 (NAP1), rather than FACT, as a histone chaperone in their studies. Altogether, their results are consistent with those of Fleming et al., that showed a role for $\mathrm{H} 2 \mathrm{Bub} 1$ in vivo in reassembling nucleosomes after passage of RNAPII but no function in depletion of nucleosomes during RNAPII passage (Fleming et al., 2008). Thus, these results on this 
topic give evidence that $\mathrm{H} 2 \mathrm{Bub} 1$ is rather a consequence of transcription and is important for events that follow the passage of RNAPII instead of directly functioning in stimulating transcriptional elongation (Kim et al., 2009).

Recently, the E3 ubiquitin ligase RNF20 was shown to function in regulating a subset of human genes (Shema et al., 2008). In these RNF20-repressed genes H2B is highly ubiquitinated and additionally marks for poised RNAPII can be found. H2Bub1 may in this case stabilize the nucleosomes downstream of RNAPII leading to a block for early elongation. The deubiquitinating enzyme USP22 could then be recruited to the paused gene by activators and function in promoting elongation via $\mathrm{H} 2 \mathrm{~B}$ deubiquitination (Espinosa, 2008). These findings thus propose a function of $\mathrm{H} 2 \mathrm{Bub} 1$ in nucleosome stability rather then opening the chromatin and can be supported by a recent model by Chandrasekharan et al., where H2Bub1 first stabilizes nucleosomes in front of RNAPII to enhance the binding of FACT. A destabilization could then be the result of deubiquitination by Ubp8p thus enhancing the function of FACT in disassembling the nucleosomes and allowing the passage of RNAPII. The newly reassembled nucleosomes upstream of RNAPII localization could then again be stabilized by H2Bub1 (Chandrasekharan et al., 2009). These two models implicate that both ubiquitination and deubiquitination of H2B are crucial for RNAPII passage and thus productive elongation and that chromatin structure plays an important part in this process.

In respect to our model in which $\mathrm{H} 2 \mathrm{Bub} 1$ functions in proper replicationdependent histone mRNA 3' end processing, the hypothesis of H2Bub1 being crucial for nucleosome reassembly rather then eviction could be explained as follows. If $\mathrm{H} 2 \mathrm{Bub} 1$ is lost as a result of CDK9 depletion, the reassembly and stabilization of nucleosomes does not take place properly anymore resulting in a nucleosome poor region that allows RNAPII to read past the normal canonical 3' end processing site until it reaches a polyadenylation signal further downstream. Like that the observed increase in polyadenylated replication-dependent histone mRNAs could be explained. A similar mechanism has been proposed for the role of chromatin structure in controlling alternative splicing (Perales and Bentley, 2009). High nucleosome occupancy or a specific histone modification pattern in exons could lead to lower elongation rate in exons in comparison to introns and could thus have an influence on splicing. It is suggested that a slow elongation rate favors the inclusion of exons whereas a fast elongation rate favors exon skipping (De la Mata et al., 2003). 


\subsubsection{Chromatin structure versus CTD-code: What gives the signal for productive transcription?}

As discussed, chromatin structure seems to play an important role in efficient transcription. However, it was shown that the phosphorylation pattern of the CTD of RNAPII serves as a platform for a variety of proteins that are implemented in premRNA capping, 3' end processing, splicing, transcription elongation, termination and chromatin modification appearing to play the major role in effective transcription (Phatnani and Greenleaf, 2006). For example, the cleavage factors CPSF73 and CSTF77 are specifically recruited to the RNAPII machinery during transcription and this is impeded if cells are treated with DRB which inhibits the phosphorylation of Ser2 of the CTD (Glover-Cutter et al., 2008). Furthermore the histone H3 chaperone SUPT6H directly interacts with phosphorylated CTD at Ser2 pointing out the importance of CTD phosphorylation in recruiting factors necessary for effective transcription (Yoh et al., 2007).

Recently, CDK8 was shown to play a positive role in regulating transcriptional elongation of genes that are part of the serum response network via phosphorylation of the CTD of RNAPII (Donner et al., 2010). CDK8 is a cyclin-dependent kinase that is associated with the Mediator complex which itself is part of the preinitiation complex and probably acts as a molecular bridge to facilitate RNAPII recruitment to the promoter (Cantin et al., 2003; Kuras and Struhl, 1999; Li et al., 1999). Although mostly characterized as a transcriptional repressor and lately identified as a potent oncoprotein, Donner et al. showed that CDK8 acts as a positive regulator of immediate-early genes (Ding et al., 2008; Firestein et al., 2008; Knuesel et al., 2009). Interestingly, siRNAmediated depletion of CDK8 resulted in a gene specific decrease in Ser2- and Ser5phosphorylation of the CTD as a result of impaired recruitment of CDK7 and CDK9. Moreover, CDK8 was shown to interact with BRD4, a bromodomain containing protein that is implicated in the recruitment of $\mathrm{P}-\mathrm{TEFb}$ (see section 1.1.2 on page 3f.) to multiple human genes, and upon knockdown of CDK8 the recruitment of BRD4 was significantly decreased (Hargreaves et al., 2009). A further observed effect of CDK8 depletion was a decrease in the elongation rate since slower elongation complexes containing hypophosphorylated RNAPII could be detected in nuclear run-on experiments. Thus, a strong correlation between CDK8 recruitment, CTD phosphorylation and RNAPII elongation rates was demonstrated (Donner et al., 2010). These results by Donner et al. assume a time-dependent function. In the early stage of 
transcription it is difficult for RNAPII to move through the gene resulting in a slow elongation rate but after ejection of some downstream nucleosomes the elongation rate could increase. Thus, a main function of the CTD of RNAPII seems to be the recruitment of proteins that change the chromatin structure in the transcribed region which is achieved by different patterns of CTD phosphorylation resulting in the recruitment of different proteins leading to a slower or faster elongation rate.

As already mentioned, the CTD of RNAPII also plays a role in mRNA 3' end processing since Ser2-phosphorylated CTD was shown to recruit 3' end processing factors to elongating RNAPII (Ahn et al., 2004; Meinhart and Cramer, 2004). Admittedly, although the CTD is necessary for efficient processing it is not sufficient since attaching the CTD onto RNAPII or T7 RNAP does not enable productive processing (Natalizio et al., 2009). So what is the importance of chromatin structure in efficient transcription? Splicing was for example shown to be connected to chromatin structure since it was shown to be influenced by chromatin remodelling ATPases (Batsche et al., 2006; Sims III et al., 2007). In addition, alternative splicing is thought to be affected by the rate of elongation since a fast elongation rate is thought to favor exon skipping whereas a slow elongation rate is suggested to favor exon inclusion (De la Mata et al., 2003). The elongation rate could in turn be defined by nucleosome occupancy or particular histone modifications (Perales and Bentley, 2009). Furthermore, during transcription positive and negative supercoiling in front of and behind the RNAPII, respectively, is established and positive supercoiling was shown to eject histone $\mathrm{H} 2 \mathrm{~A} / \mathrm{H} 2 \mathrm{~B}$ dimers from nucleosomes in vitro (Levchenko et al., 2005).

These results suggest an important role for both CTD phosphorylation and chromatin structure in efficient transcription but their specific functions and interactions still need to be further explored.

\subsubsection{A role for pausing in mRNA 3' end formation}

In most RNAPII transcribed genes, the inhibition of CDK9 results in a dramatic decrease of transcription. However, transcription of the U2 snRNA gene is only mildly affected although P-TEFb is normally recruited (Medlin et al., 2005). NELF is recruited to the end of a promoter proximal region of nucleosome depletion. Interestingly, the length of the nucleosome free region varies in different genes so that the NELF/P-TEFb elongation checkpoint can either lie close to the transcription start site or rather a few hundred base pairs further downstream. Is this checkpoint then still implicated in the 
regulation of transcriptional elongation? Medlin and co-workers showed that in the U2 snRNA this checkpoint lies at the end of the transcribed region so that $\mathrm{P}-\mathrm{TEFb}$ is not required for transcription elongation (Medlin et al., 2005). Furthermore, the same group found that NELF is implemented in the transcriptional termination of the U2 snRNA gene rather than pausing since it is only found in the end of the transcription unit (Egloff et al., 2009). Interestingly, ChIP results revealed that CDK9 could be detected throughout the whole $U 2$ gene whereas it was recruited to the $\beta$-actin gene only at the point where the histone occupancy increases. These results indicate that an increase in histone density may be important for the recruitment of NELF and suggests that chromatin structure plays a central role in deciding whether pausing of RNAPII occurs and which function this elongation checkpoint has (Egloff et al., 2009).

A question that arises from these observations is if promoter proximal pausing of RNAPII prior to productive elongation is really a process in which RNAPII halts and afterwards proceeds or if it is rather an early termination pathway and stalled RNAPII is found there because of aberrant termination and thus abortive transcription. It is not possible to distinguish between paused or early terminated RNAPII. In mammalian, Drosophila melanogaster and yeast sequencing analysis revealed the widespread existence of short, promoter-associated transcripts of which a part is rapidly degraded by the exosome indicating that they are released from the elongating RNAPII complex (Carninci, 2009; Preker et al., 2008). Buratowski thus speaks of early and late termination pathways (Buratowski, 2009). NELF has been described as a negative elongation factor that inhibits productive elongation shortly after initiation and was thus termed a 5' pausing factor (Peterlin and Price, 2006). Upon phosphorylation by CDK9, NELF leaves the elongating complex and productive elongation can proceed. Interestingly, NELF was also found to be required for proper termination of snRNAs and replication-dependent histone mRNAs (Egloff et al., 2009; Narita et al., 2007; Wagner et al., 2007). In contrast to the notion that each gene either exclusively uses an early or a late termination pathway, Buratowski proposes a kinetic competition model for termination which is dependent upon the phosphorylation status of the CTD and the timely recruitment of negative and positive transcription elongation factors (Buratowski, 2009).

While Buratowski points out the importance of CTD phosphorylation and transcription factor recruitment in termination and 3' end formation, Perales and Bentley rather hold chromatin structure responsible for these events. They underscore 
their opinion with the fact that poly(A) sites are associated with their own chromatin signature and suggest that an impact on the transcriptional elongation rate might influence 3' end processing (Perales and Bentley, 2009; Spies et al., 2009).

Interestingly, in Drosophila melanogaster a role for chromatin structure in histone mRNA 3' end processing has been proposed since the histone variants H3.3 and $\mathrm{H} 2 \mathrm{Av}$ and the histone chaperone anti-silencing function 1 (Asf1) are crucial for proper histone mRNA 3' end formation (Wagner et al., 2007). In regard to these results, our model suggests that specific CDK9-dependent phosphorylation and monoubiquitination of $\mathrm{H} 2 \mathrm{~B}$ serve as markers that allow the correct recognition of the histone mRNA 3' end cleavage site by specific mRNA 3' end processing proteins.

\subsection{Polyadenylated replication-dependent histone transcripts: formation and possible functions}

mRNAs transcribed from replication-dependent histone genes are not normally polyadenylated like all other RNAPII transcribed mRNAs but instead end in an evolutionary highly conserved 3' end stem-loop structure (Dominski and Marzluff, 1999; Marzluff et al., 2008). In addition, their transcription is cell cycle-dependent with levels increasing at the beginning of $S$ phase dropping to basal levels at the end of $S$ phase (Heintz et al., 1983; Sittman et al., 1983). Interestingly, replication-dependent histone mRNAs contain both a stem-loop structure and a downstream polyadenylation site suggesting the possibility of two pathways for the production of either canonically processed or polyadenylated mRNAs. Indeed, polyadenylated replication-dependent histone transcripts could be observed in several studies suggesting their production under certain circumstances and moreover a possible physiological role (Collart et al., 1991; Kirsh et al., 1989; Narita et al., 2007).

A recent study showed that in the absence of the tumor suppressor parafibromin, which is a component of the PAF complex, replication-dependent histone transcripts are not properly processed following the stem-loop structure but polyadenylated (Farber et al., 2010). Interestingly, the yeast Paf1p complex is necessary for the recruitment of 3' processing factors and the human PAF1 complex associates with the 3' mRNA processing factors CPSF and CSTF (Rozenblatt-Rosen et al., 2009; Sheldon et al., 2005). Since PAF plays a role in the CTD-dependent H2B monoubiquitination, these effects could, in addition, be partially due to chromatin modifications. In our studies we could investigate a connection between CDK9 activity and the status of polyadenylated 
histone transcripts as well as the fact that a $G_{1}$ cell cycle arrest, induced by p53 accumulation, serum starvation, Cyclin E knockdown or the DNA damaging reagent hydroxyurea leads to an increase in polyadenylated histone transcripts. However, the reason for the decision of the polyadenylated pathway and the mode for this switch still remain to be elucidated.

\subsubsection{Is polyadenylation of replication-dependent histone transcripts a result of splicing?}

Replication-dependent histone pre-mRNAs transcribed from canonical histone genes do not contain introns and are thus not spliced (Dominski and Marzluff, 1999, Marzluff et al., 2008). Rather, the recognition of splice junctions excludes the possibility of normal 3' end processing of replication-dependent histone mRNAs. Thus, in our studies, the recognition of splice sites in replication-dependent histone premRNAs could impede proper processing 3' to the stem-loop structure and thus lead to the usage of a downstream polyadenylation signal. Besides splicing, also spurious intragenic transcription initiation could result in the production of polyadenylated transcripts which initiate transcription downstream of the canonical histone mRNA 3' end processing site and would thus be recognized using the oligonucleotides used in the experiment illustrated in Fig I.3D,E on page 41. It was shown that by inserting one or two introns into the open reading frame of a gene containing the histone 3' end resulted in the usage of a cryptic 3' polyadenylation site and this was independent of the distance between the 3' end and the polyadenylation signal (Pandey et al., 1990). So what is the critical step in deciding whether to produce canonically processed or polyadenylated replication-dependent histone mRNAs? To address the question if splicing or spurious intragenic transcriptional initiation could be the reason for the production of polyadenylated histone mRNAs, we measured the poladenylation status of histone transcripts of a non-spliced histone gene, namely HIST2H2AA, and could detect an increase of polyadenylated histone mRNAs after siRNA mediated depletion of CDK9 (illustrated in Supp Fig I.S2A on page 52). In addition, the increase in polyadenylated transcripts of spliced histone transcripts (HIST1H2BD and HIST1H2AC) by using oligonucleotides that span the exon/exon junctions of each spliced transcript which is produced from a long primary transcript was measured (illustrated in Fig II.2A,B on page 60). No changes in the increase of polyadenylated replication-dependent histone mRNA transcripts could be observed compared to the usage of oligonucleotides that do 
not span the exon/exon junctions. These results suggest that at least our observations were not due to splicing defects and hypothesize that splicing is not the reason for the production of polyadenylated replication-dependent histone transcripts.

\subsubsection{Function and regulation of NPAT in replication-dependent histone mRNA 3' end processing.}

As already mentioned in section 1.3.2.4 on page 27f., NPAT is essential for $\mathrm{S}$ phase entry and activation of histone gene transcription (Ma et al., 2000; Wei et al., 2003; Ye et al., 2003). Thus, it is crucial for cell proliferation and proper cell cycle regulation. In addition to NPAT, another protein has been shown to play an important role in the regulation of histone transcription. FADD-like IL-1 $\beta$-converting enzyme (FLICE) associated huge protein (FLASH) was initially identified as a component of the apoptosis signalling complex called the death-inducing signalling complex (DISC) (Imai et al., 1999). However, its role in activation of caspase 8 has been questioned since the existence of domains mediating interactions between proteins of the apoptosis machinery could not be confirmed (Koonin et al., 1999). Despite descriptions of being a cytoplasmic protein, FLASH was found in subnuclear foci of the nucleus, more precisely it was shown to colocalize with coilin and NPAT to Cajal bodies (Barcaroli et al., 2006b). Interestingly, FLASH and NPAT interact in vivo independent of coilin and are both localized to histone locus bodies (HLBs). Moreover, they are both important for proper $\mathrm{S}$ phase expression of histone genes and required for maintenance of Cajal body structure. However, despite these similarities, they do not share any significant regions of sequence similarity and more importantly, depletion of NPAT leads to an inhibition of S phase entry whereas depletion of FLASH blocks progression through $\mathrm{S}$ phase (Barcaroli et al., 2006a; Ye et al., 2003).

Recently, the N-terminus of FLASH was found to interact with the U7 snRNP component LSM11 (Yang et al., 2009). Furthermore, depletion of the ortholog of human FLASH in Drosophila melanogaster (dFLASH) resulted in polyadenylation of replication-dependent histone pre-mRNAs indicating a role for FLASH in proper 3' end processing of histone pre-mRNA (Yang et al., 2009). Since the stimulation of 3' end cleavage is not dependent upon SLBP and a more efficient recruitment of U7 snRNP to the cleavage site, Yang et al. hypothesize that FLASH interacts with LSM11 and with an unidentified component of the processing machinery that recruits or activates the 3 ' endonuclease CPSF73. 
Our results showed that siRNA mediated depletion of NPAT resulted in a loss of proper replication-dependent histone mRNA 3' end processing and led to polyadenylation of these transcripts (illustrated in Fig III.5A,B on page 79). The similar functions of FLASH and NPAT and the fact that FLASH is implicated in histone mRNA 3' end processing supports our data and leads to the hypothesis that NPAT not only plays a role in histone transcription but is also important for proper histone mRNA 3 ' end processing. To test the hypothesis if its role is even more related to proper processing than to regulation of histone transcription one could add a constitutive not cell cycle-regulated promoter to a histone gene and look for recruitment of NPAT. Since NPAT is believed to be recruited through factors that are histone specific, binding of NPAT to this artificial construct would suggest that NPAT is rather implemented in processing than in transcription.

As already mentioned in section 1.3.2.4 on page 27f., NPAT is phosphorylated by Cyclin E/CDK2 and this leads to stimulation of NPAT's ability to promote S phase entry and histone gene transcription (Ma et al., 2000; Zhao et al., 1998). In the Nterminus a LisH-like motif was found to be critical for the ability of NPAT to activate histone gene expression but not important for promoting $\mathrm{S}$ phase entry (illustrated in Fig 7 on page 106) (Wei et al., 2003). Moreover, studies on the sequence of NPAT revealed a RXL-like motif which is known to facilitate interaction with cyclins in other CDK substrates (illustrated in Fig 7 on page 106) (Adams et al., 1996; Schulman et al., 1998; Zhu et al., 1995).

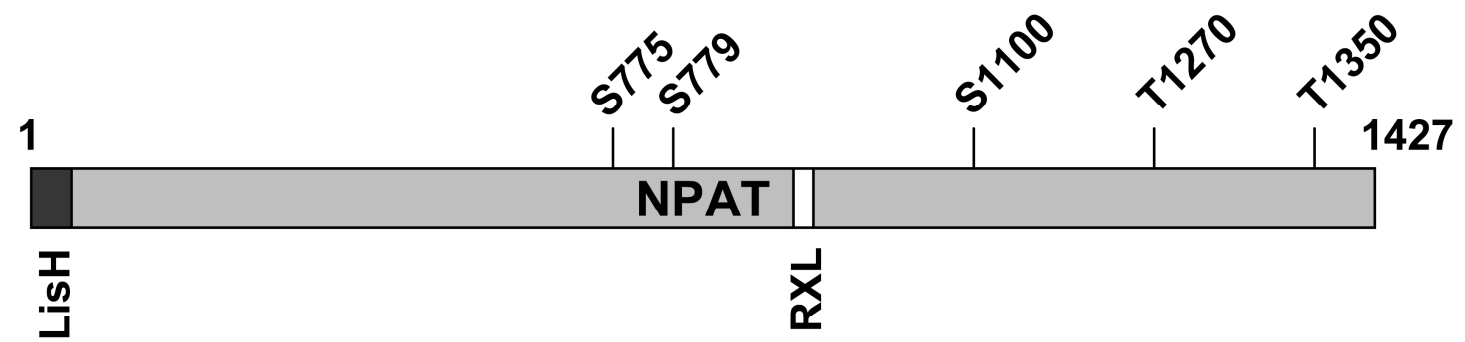

(3-35) $(1062-1066)$

Fig 7: Schematic diagram of NPAT. Shown are the N-terminal LisH-domain, the RXL-domain (the cyclin interaction motif) and the five known CDK phosphorylation sites (modified from Wei et al., 2003).

Our GST-pulldown experiment revealed an in vitro interaction of an NPAT fragment (amino acids 801-1100) that contains the RXL-domain (amino acids 10621066) with the P-TEFb components Cyclin T1 and CDK9 (illustrated in Fig III.5D on 
page 79). Furthermore, immunoprecipitated endogenous NPAT could coimmunoprecipitate Cyclin T1 as well as CDK9 (illustrated in Fig III.5E on page 79). Our model hypothesizes that NPAT recruits $\mathrm{P}-\mathrm{TEFb}$ to histone gene promoters which then promotes transcription by phosphorylation of Ser2 of the RNAPII CTD, NELF and SUPT5H, and promotes processing through the recruitment of histone processing enzymes through modifications to chromatin structure (illustrated in Fig. III.6D,E on page 81). Taking into consideration that CDK9 is a Ser/Thr kinase and that the NPAT protein contains at least 5 phosphorylation sites (Wei et al., 2003) one could speculate if CDK9 also phosphorylates NPAT as well. In this scenario NPAT could recruit P-TEFb via direct interactions and would then itself be regulated through phosphorylation by CDK9.

Accumulation of the cell cycle inhibitor p21 via Nutlin-3a treatment that inhibits the interaction of MDM2 with p53 and thus leads to stabilization and accumulation of p53, resulted in a decrease of NPAT protein expression (illustrated in Fig III.3E on page 75). Interestingly, it seems that before a decrease of the overall protein expression occurred, the phosphorylation status of NPAT was reduced. We hypothesize that the E2F/RB pathway directly regulates NPAT expression but that the p21-dependent inhibition of CDKs regulate the phosphorylation status of NPAT which is a quicker effect than the overall transcriptional regulation. It could well be that unphosphorylated NPAT is recruited to the promoter of a histone gene and is then activated upon phosphorylation by Cyclin E/CDK2 (or perhaps CDK9 as hypothesized above).

As a CDK inhibitory protein (CKI) p21 directly binds to Cyclin E/CDK2 and blocks its activity thus preventing phosphorylation of RB and keeping E2F inactive (Sherr and Roberts, 2004). The same is true for the CKIs p27 and p57 although their functions in mammalian development are distinct (Nakayama and Nakayama, 1998; Sherr and Roberts, 2004). Interestingly, p21 seems to be less effective in inhibiting the Cyclin E/CDK2-dependent phosphorylation of NPAT compared to p27 and p57, more precisely, p57 was shown to be the most effective inhibitor of the Cyclin E/CDK2/NPAT/HINFP pathway which may be explained by its ability to directly form a complex with NPAT (Mitra et al., 2009).

A recent study also revealed a function of p57 in negatively regulating the phosphorylation of the CTD of RNAPII in an E2F1-dependent manner (Ma et al., 2010). Interestingly, in this study p57 was shown to interact with CDK7 and CDK9 in vivo and to inhibit both Ser2- and Ser5-phosphorylation of the CTD. Furthermore, E2F1 
was required for p57 to block CTD phosphorylation and E2F1 and p57 were found to directly interact and form stable complexes that can be detected on E2F-regulated promoters. Notably, the detected activity of p57 was not an indirect consequence of regulating $\mathrm{RB}$ phosphorylation since HeLa cells which were amongst others used in these studies express the E7 oncogene that inactivates RB. Ma et al. drew a model in which p57 binds to E2F associated with an E2F-regulated promoter and inhibits the phosphorylation of the CTD of RNAPII at P-Ser2 and P-Ser5 resulting in suppression of the transcription of E2F target genes.

Since our studies revealed the dependence of monoubiquitination of histone $\mathrm{H} 2 \mathrm{~B}$ on CDK9 activity, massive changes in $\mathrm{H} 2 \mathrm{Bub} 1$ levels should be expected to occur after accumulation of the CDK inhibitor p57 through CDK9 inhibition. Taking into account the well known tumor suppressor function of p57, a change in H2Bub1 levels would point out a more direct connection between posttranslational histone modifications and cell cycle regulation resulting in modification of the chromatin structure and tumor suppression.

\subsection{3 $G_{0} / G_{1}$ cell cycle arrest but not a $G_{2}$ arrest seems to be relevant for the production of polyadenylated histone mRNAs}

Our results show an increase in polyadenylated replication-dependent histone transcripts upon induction of a $G_{1}$ cell cycle arrest. This could either be achieved by accumulation of p53 upon treatment with the chemical MDM2 inhibitor Nutlin-3a, by serum-starvation, DNA damage induction via HU treatment or siRNA mediated depletion of Cyclin E1 or Cyclin D1 in the colorectal cancer cell line HCT116 (illustrated in Fig III.1C,D on page 70, Fig III.4B,E,F on page 77, Supp Fig III.S6 on page 91 and data not shown). But is a $G_{1}$ arrest crucial for an increase of polyadenylated replication-dependent histone transcripts or could any other cell cycle arrest like, for example, $\mathrm{G}_{2}$ arrest have the same effects? Interestingly, the effects in HCT116 cells observed upon Nutlin-3a treatment could not be observed in the human osteosarcoma cell line U2 OS although this cell line is also wildtype for p53. Our cell cycle analysis revealed that after p53 accumulation U2 OS cells do not show a distinct $\mathrm{G}_{1}$ arrest like HCT116 cells but rather go into $G_{1}$ and $G_{2}$ phase evenly (data not shown). Thus, a general cell cycle arrest is not sufficient to generate the effects observed in HCT116 cells. In $\mathrm{G}_{1}$ phase the cell gets ready for its function in $\mathrm{S}$ phase, namely DNA replication where histones are needed. Afterwards, when cells enter $G_{2}$ phase there is no need for 
the production of histones anymore and moreover cells do not remain for a long time in this cell cycle phase. Importantly, there are mechanisms in place which prevent histone expression like the degradation of histone transcripts, NPAT and SLBP. Thus, it would make more sense to produce additional histone transcripts if a $G_{1}$ arrest occurs than if cells go into $G_{2}$ arrest. It also remains possible that Nutlin-3a might put HCT116 cells into $G_{0}$ phase, meaning permanent cell cycle arrest instead of a $G_{1}$ arrest. This would make sense since quiescent cells or terminally differentiated cells reside in $G_{0}$ phase and could thus make use of polyadenylated histone transcripts over time as histones become degraded and need to be replaced. Unfortunately, a $\mathrm{G}_{0}$ arrest can not be distinguished from a $\mathrm{G}_{1}$ arrest by common flow cytometric analysis. However, by removal of Nutlin3a subsequent to treatment one could examine if the cells are able to reenter the cell cycle and thus answer the question if cells are put into permanent cell cycle arrest or not.

Since siRNA mediated depletion of either Cyclin E1 or Cyclin D1 in HCT116 p53-/- cells that are null for p53 revealed the same results as in HCT116 p53 wildtype cells, namely an increase in polyadenylated histone mRNAs, the regulation of the switch between the canonical histone processing and polyadenylation pathways seem to be independent of p53 itself but due to cell cycle arrest.

\subsubsection{The diverse mechanisms by which CDK9 controls histone mRNA 3' end processing}

In our studies knockdown of CDK9, NELF-E, SUPT5H, the PAF component CTR9, Cyclin T1, RNF20 or RNF40 resulted in an increase of polyadenylated histone mRNAs produced from replication-dependent histone genes. Interestingly, although the knockdowns were comparably efficient, siRNA mediated depletion of CDK9 resulted in an approximately 15-fold increase whereas RNF20- or RNF40-knockdown led to only a two-fold increase in polyadenylated histone mRNAs (illustrated in Fig I.3D,E on page 41). Since H2Bub1 is a direct target of RNF20/40 activity it seems to not be the most downstream target of CDK9 which affects histone mRNA processing. As already mentioned, CDK9 has multiple targets that could play a role in controlling proper replication-dependent histone mRNA 3' end processing. Since knockdown of NELF-E and SUPT5H also resulted in an increase of polyadenylated histone transcripts and both are targets of CDK9, the larger effect of CDK9 knockdown on the production of polyadenylated histone mRNAs is likely a sum of each event. CDK9 knockdown thus 
probably affects not only the CTD-dependent regulation of $\mathrm{H} 2 \mathrm{Bub} 1$ but also the function of SUPT5H and NELF-E in proper histone mRNA 3' end processing. Interestingly, in our studies siRNA-mediated depletion of SUPT5H had almost no effect on $\mathrm{H} 2 \mathrm{Bub}$ and $\mathrm{H} 3 \mathrm{~K} 4 \mathrm{me} 3$ but led to a considerable decrease of H3K36me3 protein levels. In addition, an approximately 6-fold increase of polyadenylated histone mRNAs could be detected. This confirms the suggestion that polyadenylation of histone transcripts can occur independent of H2Bub1 as well. Since CDK9 depletion affects not only $\mathrm{H} 2 \mathrm{Bub} 1$ and $\mathrm{H} 3 \mathrm{~K} 4 \mathrm{me} 3$ but also $\mathrm{H} 3 \mathrm{~K} 36 \mathrm{me} 3$, H3K36me 3 could also play a role in controlling the polyadenylation of histone mRNAs. The effect of depletion of the H3K36 methylating protein SETD2 on histone mRNA polyadenylation confirms this (illustrated in Fig II.2C on page 60). Despite a possible RNAPII CTD-independent function of $\mathrm{H} 3 \mathrm{~K} 36 \mathrm{me} 3$ in polyadenylation of histone mRNAs, a function of the capping enzyme for the recruitment of 3' end processing factors should also be considered. Since SUPT5H interacts with the capping enzyme (CE), a depletion of SUPT5H could result in less recruitment of the $\mathrm{CE}$ and thus less recruitment of the 3' end processing machinery. A function for the $\mathrm{CE}$ in elongation, termination and 3' end processing has already been suggested (Adamson et al., 2005; Glover-Cutter et al., 2008; Perales and Bentley, 2009). Interestingly, a recent study in Schizosaccharomyces pombe shows that the CTD of Spt5p and RNAPII have functionally overlapping roles in capping enzyme recruitment (Schneider et al., 2010). Shortening of Spt5p CTD resulted in growth defects and this could be rescued by coexpressing RNAPII CTD. More interestingly, the mutation of both CTDs had an even more severe effect and suggested that Spt5p CTD and RNAPII CTD act synergistically. However, since phosphorylation of RNAPII CTD at Ser2 and/or Spt5p CTD on Thr1 are not essential for viability of S. pombe, CDK9 must phosphorylate another essential target site in vivo. Nevertheless, these data support both RNAPII CTD-dependent and -independent functions of CDK9 activity.

Recently, NELF and CBC80 have been shown to play an important role in proper 3' end processing of replication-dependent histone transcripts since their knockdown resulted in an increase in polyadenylated histone mRNAs (Narita et al., 2007). The effect of siRNA mediated depletion of NELF on the production of polyadenylated replication-dependent histone transcripts was repeated and confirmed in our studies (illustrated in Supp Fig I.S2B on page 52). Interestingly, NELF is generally considered to play an opposing role to CDK9 in transcriptional regulation but knockdown of either protein similarly led to an increase in polyadenylated histone 
transcripts. How could this be explained? Probably these two proteins play distinct roles during different cell cycle phases. For example, NELF might repress the expression of replication-dependent histone transcripts outside of $\mathrm{S}$ phase and CDK9 could, in contrast, play a role in ensuring proper processing of replication-dependent histone mRNAs during DNA replication. In case of a knockdown of either protein, derepression of histone transcripts outside of S phase in the absence of SLBP (following NELF-E knockdown) or deregulation of proper processing during $\mathrm{S}$ phase (following CDK9 knockdown) would similarly result in an increase of polyadenylated histone mRNAs in asynchronous cell cultures. Since this is only a speculation further studies in synchronized cell cultures would be necessary to proof this hypothesis. For example, one could synchronize cells and examine the presence of NELF and CDK9 in each cell cycle phase. Moreover, synchronized cells in which NELF or CDK9 has been depleted by siRNA transfection could be harvested in different cell cycle phases and tested for the amount of polyadenylated histone transcripts to determine if the effects of NELF and CDK9 knockdown are indeed cell cycle phase specific.

Interestingly, using a high-throughput yeast two-hybrid system a binary proteinprotein interaction of the nuclear cap binding protein subunit 1 (NCBP1) also known as CBP80 and RNF40 was detected (Rual et al., 2005). Based on these results one could speculate if NELF, CBP80 and RNF20/40 could somehow collaborate in the regulation of replication-dependent histone mRNA 3' end processing for example in such a way that NELF and CBP80 could recruit RNF20/40 to transcribed histone genes. The activation of RNF20/40 could then require CTD binding factors that are recruited upon CTD phosphorylation. Thus, an only partial effect of each individual in regulating replication-dependent histone mRNA 3' end processing may perhaps be explained.

\subsubsection{What is the molecular mechanism which controls the mode of replication-dependent histone mRNA 3' end processing?}

As already mentioned, in addition to depletion of CDK9, RNF20/40, NELF, CBP80 or SLBP, the induction of $\mathrm{a}_{1}$ arrest increased the production of polyadenylated histone transcripts as well. This could be shown by a $\mathrm{G}_{1}$ arrest induced by activating the p53 pathway, serum starvation, DNA damage reagents or Cyclin E depletion (illustrated in Fig III.1C,D on page 70, Fig III.4B,E,F on page 77, Supp Fig III.S6 on page 91, Supp Fig III.7B,C on page 92). 
Cells are arrested in $\mathrm{G}_{1}$ phase if they experience stress, DNA damage or other harms that would make proper DNA replication unfavorable. Thus, either repair mechanisms are necessary or if damage is too severe the cell goes into apoptosis. Since the transcription of replication-dependent histone genes is restricted to $S$ phase and histone mRNAs have very short half-lives, there is only a very low basal level of histone transcripts produced in other cell cycle phases. If cells experience DNA damage for example, histones need to be exchanged and thus it is reasonable that in this case polyadenylated histone transcripts produced outside of S phase which exhibit cell cycleindependent stability and translation might be advantageous (Kirsh et al., 1989). What exactly the molecular signal is that leads to the production of histone transcripts without a 3' end stem-loop structure but instead containing a poly(A) tail is not known. In fact, a signal for the production of polyadenylated histone mRNAs may not be necessary if a low basal level of histone gene transcription occurs in the absence of canonical 3 ' end processing factors since these transcripts would likely become polyadenylated if not recognized by cell cycle-regulated processing factors such as NPAT or SLBP.

Our results showed that depletion of CDK9 resulted in an increase in the production of polyadenylated histone transcripts and importantly less recruitment of SLBP and LSM11 to the cleavage site of the examined histone gene (illustrated in Fig I.4D on page 42). This observation leads to the suggestion that if proper 3' end processing can not occur because of the lack of crucial processing factors like SLBP and LSM11 as part of the U7 snRNP, polyadenylated histone transcripts are produced. Since the expression of SLBP is restricted to S phase but absolutely necessary for transport, stabilization and translation of processed replication-dependent histone transcripts (Marzluff et al., 2008), cells that experience cell cycle arrest would not be able to produce histones unless they would choose a second, SLBP-independent, polyadenylation pathway.

Knockdown of SLBP in human cells was shown to result in an increase of cells in S phase and reduction of cell proliferation (Sullivan et al., 2009). Interestingly, it was observed that most of the histone transcripts are properly expressed but are retained in the nucleus pointing out a role for SLBP in histone mRNA export. Does that mean that the polyadenylated histone mRNA transcripts that we observed in our studies are not being translated? Since polyadenylated histone mRNA transcripts were found on polyribosomes in mammalian cells this indicates that they are indeed translated and that polyadenylation does not affect their translatability but rather likely allows for cell 
cycle-independent transport and translation (Kirsh et al., 1989; Moss et al., 1994). However, the importance of polyadenylated histone transcripts still needs to be investigated.

\subsubsection{Possible physiological functions of polyadenylated replication- dependent histone mRNA transcripts}

As already mentioned, in addition to normal replication-dependent histone transcripts that end in a 3' end stem-loop structure, replication-dependent histone mRNAs containing a poly(A) tail have also been reported (Challoner et al., 1989; Cheng et al., 1989; Collart et al., 1991; Kirsh et al., 1989; Moss et al., 1994). Thus, mammalian histone genes contain signals for the formation of a stem-loop structure as well as signals for the production of a poly(A) tail. Why then, if polyadenylated histone transcripts are thought to just be a result of processing defects, has the possibility of either producing transcripts containing a stem-loop or a poly(A) tail been conserved throughout evolution? If polyadenylated histone transcripts had no physiological role, they would not be produced, but under certain conditions they are indeed (Collart et al., 1991; Kirsh et al., 1989; Narita et al., 2007). But what is the physiological function of polyadenylated mRNAs produced from replication-dependent histone genes?

Their increased stability due to their poly(A) tail and their cell cycle-independent translation (Kirsh et al., 1989) could provide an advantage to non-dividing normal cells or rapidly dividing tumor cells. Long living terminally differentiated cells like nerve cells, muscle cells, osteocytes or memory B cells live without being replicated. For maintaining proper DNA packaging and chromatin structure in the absence of DNA replication, for example when histones are degraded by natural processes, there would be no possible source of new histones if all histone synthesis were replicationdependent. Polyadenylated histone transcripts instead could be translated replicationindependently and thus provide a source of histones for non-dividing cells. Since these are only speculations, it would be interesting to investigate if such cell types produce polyadenylated histone transcripts under specific circumstances like differentiation or tumorigenesis as well.

Furthermore, microarray analysis revealed changes in the expression of polyadenylated replication-dependent histone mRNAs connected to cancer (Abba et al., 2005; Ehlers et al., 2008; Martinez et al., 2007; Zhao et al., 2004). In estrogen receptoralpha positive breast cancers the polyadenylated histone transcript of the HISTIH2BD 
gene was for example shown to be overexpressed and in invasive lobular carcinomas (ILCs) the expression of 11 different histone gene transcripts were downregulated (Abba et al., 2005; Zhao et al., 2004). Another study identified a downregulation in the expression of the polyadenylated replication-dependent histone transcripts of the HIST1H2BD and HIST1H2BG genes in human papilloma virus positive (HPV+) head and neck squamous cell carcinomas in comparison to HPV negative (HPV-) ones (Martinez et al., 2007). Taking the observation of polyadenylated replication-dependent histone transcripts being differentially expressed in cancers in comparison to normal tissues into consideration, polyadenylated histone mRNAs could provide an advantage to rapidly dividing cells by supplying an additional source of histones translated outside of $\mathrm{S}$ phase. Both terminally differentiated cells or rapidly dividing cancer cells would thus gain an advantage in producing additional histones translated outside of $\mathrm{S}$ phase.

To gain more knowledge about the biological significance of polyadenylated histone transcripts it would be interesting to investigate which histone transcripts are most highly polyadenylated. By utilizing siRNA-mediated depletion of those specific mRNAs one could treat cells with or without chemotherapeutics and afterwards perform proliferation assays, colony formation assays and flow cytometry analysis to check, for example, for physiological differences in transcription, survival and genomic stability.

Finally, the evolutionary conserved existence of downstream polyadenylation signals in replication-dependent histone genes points to a usage and function and further studies will aim to investigate the physiological role of a switch from canonical histone mRNA 3' end processing towards polyadenylation. 


\section{Summary and Conclusions}

In the presented studies we investigated a connection between CTD phosphorylation and posttranslational histone modifications and identified new mechanisms for the production of polyadenylated replication-dependent histone transcripts. CDK9 activity that leads to phosphorylation of Ser2 of RNAPII CTD was shown to be crucial for maintaining overall H2Bub1 levels and moreover H3K4me3 and H3K36me3 levels. Besides Ser2 other CDK9 targets like SUPT5H, NELF-E and probably the human homolog of yeast Rad6p seem to be implicated in these histone modifications. Interestingly, CDK9 activity and $\mathrm{H} 2 \mathrm{Bub} 1$ help to maintain proper histone mRNA 3' end processing since depletion of CDK9 resulted in less recruitment of specific mRNA 3' end processing factors to histone genes leading to read-through of RNAPII to an alternative downstream polyadenylation signal and thus production of polyadenylated replication-dependent histone mRNAs. We could further show that the induction of a $\mathrm{G}_{1} / \mathrm{G}_{0}$ cell cycle arrest following p53 activation, serum starvation, treatment with the DNA damaging reagent hydroxyurea (HU) or following Cyclin E1 depletion leads to a decrease in total histone gene expression and an increase in polyadenylated histone transcripts. Inducing a $\mathrm{G}_{1} / \mathrm{G}_{0}$ arrest negatively affected E2Fdependent transcription of the histone-specific transcriptional regulator NPAT. We could show that NPAT can directly interact with P-TEFb (CDK9/Cyclin T1) via its cyclin binding RXL motif and created a model in which NPAT recruits P-TEFb to histone genes to ensure proper 3' end processing (illustrated in Fig 8 on page 116f.). Thus we provide evidence that $\mathrm{G}_{1} / \mathrm{G}_{0}$ arrest alters replication-dependent histone 3 ' end processing leading to the production of polyadenylated histone transcripts. We hypothesize that the production of additional histone transcripts outside of $\mathrm{S}$ phase may provide an advantage for quiescent or terminally differentiated cells by serving to maintain proper DNA packaging and chromatin structure in the absence of DNA replication. 


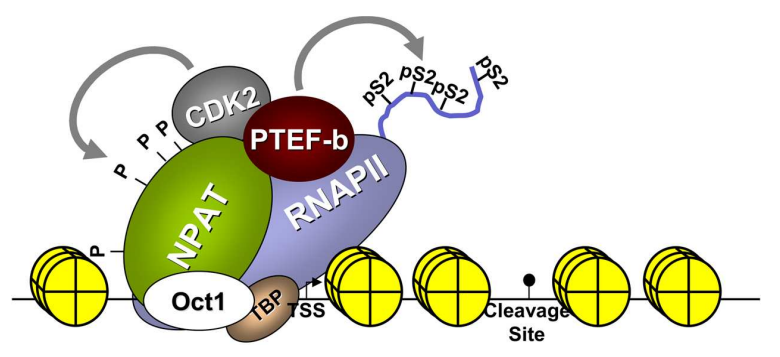

$\underline{\text { Normal cell cycle progression }}$ (S phase)
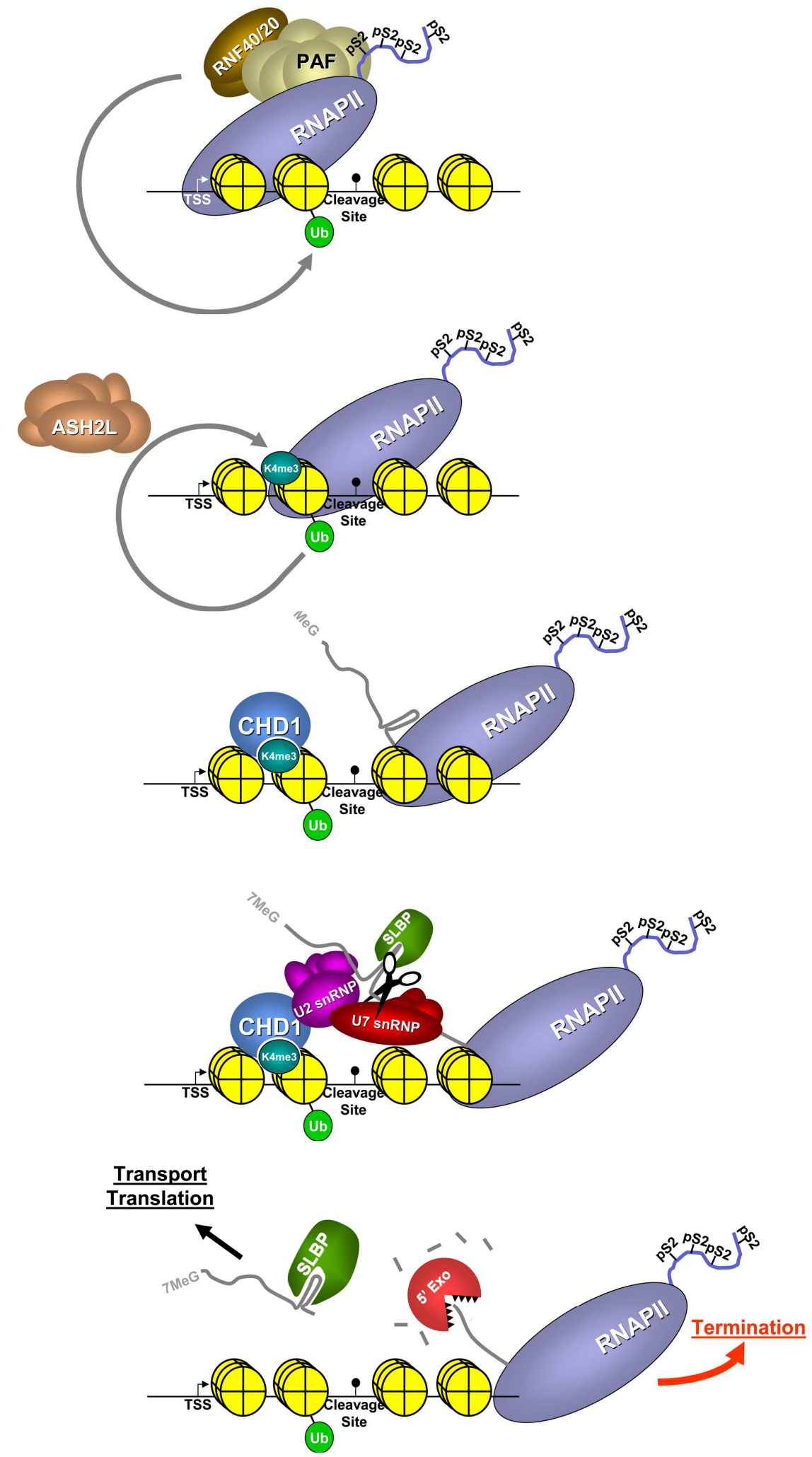


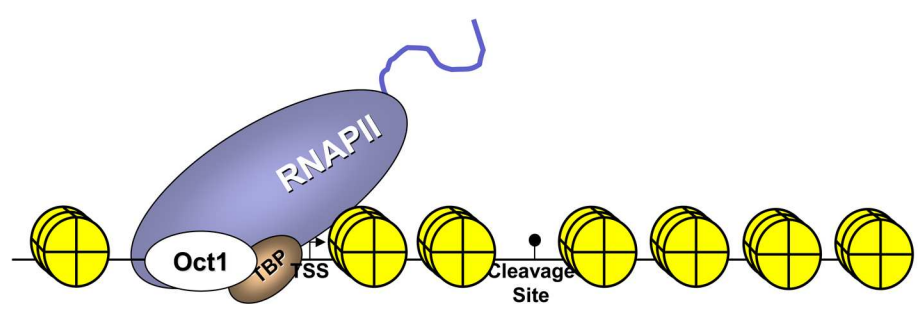

$\underline{\text { Upon } \mathrm{G}_{1} / \underline{\mathrm{G}}_{0} \text { cell cycle arrest }}$

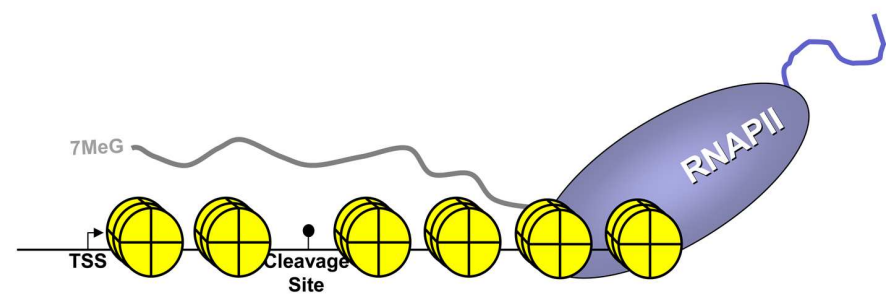

Fig 8: Schematic representation for the role of $G_{1} / G_{0}$ arrest in controlling the mode of replicationdependent histone 3' end formation. (A) In the beginning of $S$ phase NPAT binds to histone gene promoters via factors like OCT1, gets phosphorylated and thus activated by Cyclin E/CDK2 and recruits $\mathrm{P}-\mathrm{TEFb}$. CDK9 functions in phosphorylation of Ser2 of RNAPII CTD leading to the recruitment of PAF and to monoubiquitination of histone H2B by RNF20/40. H2Bub1 is known to be a prerequisite for ASH2L-dependent H3K4me3 that is recognized and bound by CHD1 which can recruit U2 snRNP that functions together with U7 snRNP and SLBP in proper 3' end processing and termination. (B) Upon $\mathrm{G}_{1} / \mathrm{G}_{0}$ arrest NPAT expression is decreased leading to less recruitment of NPAT and P-TEFb finally resulting in mistaking the 3 ' end cleavage site and thus readthrough of RNAPII to a downstream polyadenylation site (composed from the models in publication II and III). 


\section{Reference List}

Abba, M.C., Hu, Y., Sun, H., Drake, J.A., Gaddis, S., Baggerly, K., Sahin, A., and Aldaz, C.M. (2005). Gene expression signature of estrogen receptor alpha status in breast cancer. BMC. Genomics 6, 37.

Abbas, T. and Dutta, A. (2009). p21 in cancer: intricate networks and multiple activities. Nat. Rev. Cancer 9, 400-414.

Adams, P.D., Sellers, W.R., Sharma, S.K., Wu, A.D., Nalin, C.M., and Kaelin, W.G. Jr. (1996). Identification of a cyclin-cdk2 recognition motif present in substrates and p21-like cyclin-dependent kinase inhibitors. Mol. Cell Biol. 16, 6623-6633.

Adamson, T.E., Shutt, D.C., and Price, D.H. (2005). Functional coupling of cleavage and polyadenylation with transcription of mRNA. J. Biol. Chem. 280, 3226232271.

Agalioti, T., Chen, G., and Thanos, D. (2002). Deciphering the transcriptional histone acetylation code for a human gene. Cell 111, 381-392.

Ahn, S.H., Kim, M., and Buratowski, S. (2004). Phosphorylation of serine 2 within the RNA polymerase II C-terminal domain couples transcription and 3' end processing. Mol. Cell 13, 67-76.

Akhtar, M.S., Heidemann, M., Tietjen, J.R., Zhang, D.W., Chapman, R.D., Eick, D., and Ansari, A.Z. (2009). TFIIH kinase places bivalent marks on the carboxyterminal domain of RNA polymerase II. Mol. Cell 34, 387-393.

Allan, J., Hartman, P.G., Crane-Robinson, C., and Aviles, F.X. (1980). The structure of histone $\mathrm{H} 1$ and its location in chromatin. Nature 288, 675-679.

Almeida, F., Saffrich, R., Ansorge, W., and Carmo-Fonseca, M. (1998). Microinjection of anti-coilin antibodies affects the structure of coiled bodies. J. Cell Biol 142, 899912.

Andersson, R., Enroth, S., Rada-Iglesias, A., Wadelius, C., and Komorowski, J. (2009). Nucleosomes are well positioned in exons and carry characteristic histone modifications. Genome Res. 19, 1732-1741.

Aressy, B. and Ducommun, B. (2008). Cell cycle control by the CDC25 phosphatases. Anticancer Agents Med. Chem. 8, 818-824.

Arnold, D.R., Francon, P., Zhang, J., Martin, K., and Clarke, H.J. (2008). Stem-loop binding protein expressed in growing oocytes is required for accumulation of mRNAs encoding histones $\mathrm{H} 3$ and $\mathrm{H} 4$ and for early embryonic development in the mouse. Dev. Biol 313, 347-358.

Azzouz, T.N., Gruber, A., and Schümperli, D. (2005). U7 snRNP-specific Lsm11 protein: dual binding contacts with the $100 \mathrm{kDa}$ zinc finger processing factor (ZFP100) and a ZFP100-independent function in histone RNA 3' end processing. Nucleic Acids Res. 33, 2106-2117. 
Baker, S.J., Fearon, E.R., Nigro, J.M., Hamilton, S.R., Preisinger, A.C., Jessup, J.M., van Tuinen, P., Ledbetter, D.H., Barker, D.F., Nakamura, Y., White, R., and Vogelstein, B. (1989). Chromosome 17 deletions and p53 gene mutations in colorectal carcinomas. Science 244, 217-221.

Bandara, L.R., Buck, V.M., Zamanian, M., Johnston, L.H., and La Thangue, N.B. (1993). Functional synergy between DP-1 and E2F-1 in the cell cycle-regulating transcription factor DRTF1/E2F. EMBO J. 12, 4317-4324.

Barcaroli, D., Bongiorno-Borbone, L., Terrinoni, A., Hofmann, T.G., Rossi, M., Knight, R.A., Matera, A.G., Melino, G., and De Laurenzi, V. (2006a). FLASH is required for histone transcription and S-phase progression. Proc. Natl. Acad. Sci. U. S. A $103,14808-14812$.

Barcaroli, D., Dinsdale, D., Neale, M.H., Bongiorno-Borbone, L., Ranalli, M., Munarriz, E., Sayan, A.E., McWilliam, J.M., Smith, T.M., Fava, E., Knight, R.A., Melino, G., and De Laurenzi, V. (2006b). FLASH is an essential component of Cajal bodies. Proc. Natl. Acad. Sci. U. S. A 103, 14802-14807.

Barski, A., Cuddapah, S., Cui, K., Roh, T.Y., Schones, D.E., Wang, Z., Wei, G., Chepelev, I., and Zhao, K. (2007). High-resolution profiling of histone methylations in the human genome. Cell 129, 823-837.

Batsche, E., Yaniv, M., and Muchardt, C. (2006). The human SWI/SNF subunit Brm is a regulator of alternative splicing. Nat. Struct. Mol. Biol. 13, 22-29.

Bell, O., Wirbelauer, C., Hild, M., Scharf, A.N., Schwaiger, M., MacAlpine, D.M., Zilbermann, F., van Leeuwen, F., Bell, S.P., Imhof, A., Garza, D., Peters, A.H., and Schubeler, D. (2007). Localized H3K36 methylation states define histone H4K16 acetylation during transcriptional elongation in Drosophila. EMBO J. 26, 49744984.

Belotserkovskaya, R. and Reinberg, D. (2004). Facts about FACT and transcript elongation through chromatin. Curr. Opin. Genet. Dev. 14, 139-146.

Bentley, D. (2002). The mRNA assembly line: transcription and processing machines in the same factory. Curr. Opin. Cell Biol. 14, 336-342.

Bentley, D.L. (2005). Rules of engagement: co-transcriptional recruitment of premRNA processing factors. Curr. Opin. Cell Biol. 17, 251-256.

Berger, S.L. (2002). Histone modifications in transcriptional regulation. Curr. Opin. Genet. Dev. 12, 142-148.

Berger, S.L. (2007). The complex language of chromatin regulation during transcription. Nature 447, 407-412.

Bernstein, B.E., Humphrey, E.L., Erlich, R.L., Schneider, R., Bouman, P., Liu, J.S., Kouzarides, T., and Schreiber, S.L. (2002). Methylation of histone H3 Lys 4 in coding regions of active genes. Proc. Natl. Acad. Sci. U. S. A 99, 8695-8700.

Bernstein, B.E., Mikkelsen, T.S., Xie, X., Kamal, M., Huebert, D.J., Cuff, J., Fry, B., Meissner, A., Wernig, M., Plath, K., Jaenisch, R., Wagschal, A., Feil, R., Schreiber, 
S.L., and Lander, E.S. (2006). A bivalent chromatin structure marks key developmental genes in embryonic stem cells. Cell 125, 315-326.

Besson, A., Dowdy, S.F., and Roberts, J.M. (2008). CDK inhibitors: cell cycle regulators and beyond. Dev. Cell 14, 159-169.

Birnstiel, M.L., Busslinger, M., and Strub, K. (1985). Transcription termination and 3' processing: the end is in site! Cell 41, 349-359.

Birnstiel, M.L., Schaufele, F.J., (1988). Structure and function of minor snRNPs. In: Birnstiel, M.L. (Ed.), Structure and function of major and minor small ribonucleoprotein particles. Springer-Verlag, Berlin, pp. 155-182.

Boeing, S., Rigault, C., Heidemann, M., Eick, D., and Meisterernst, M. (2010). RNA polymerase II C-terminal heptarepeat domain Ser-7 phosphorylation is established in a mediator-dependent fashion. J. Biol. Chem. 285, 188-196.

Bongiorno-Borbone, L., De Cola, A., Barcaroli, D., Knight, R.A., Di Ilio, C., Melino, G., and De Laurenzi, V (2010). FLASH degradation in response to UV-C results in histone locus bodies disruption and cell-cycle arrest. Oncogene 29, 802-810.

Bongiorno-Borbone, L., De Cola, A., Vernole, P., Finos, L., Barcaroli, D., Knight, R.A., Melino, G., and De Laurenzi, V (2008). FLASH and NPAT positive but not Coilin positive Cajal Bodies correlate with cell ploidy. Cell Cycle 7, 2357-2367.

Bres, V., Yoh, S.M., and Jones, K.A. (2008). The multi-tasking P-TEFb complex. Curr. Opin. Cell Biol 20, 334-340.

Briggs, S.D., Xiao, T., Sun, Z.W., Caldwell, J.A., Shabanowitz, J., Hunt, D.F., Allis, C.D., and Strahl, B.D. (2002). Gene silencing: trans-histone regulatory pathway in chromatin. Nature 418, 498.

Brookes, E. and Pombo, A. (2009). Modifications of RNA polymerase II are pivotal in regulating gene expression states. EMBO Rep. 10, 1213-1219.

Brown, M.A., Sims, R.J. III, Gottlieb, P.D., and Tucker, P.W. (2006). Identification and characterization of Smyd2: a split SET/MYND domain-containing histone H3 lysine 36-specific methyltransferase that interacts with the $\operatorname{Sin} 3$ histone deacetylase complex. Mol. Cancer 5, 26.

Brush, D., Dodgson, J.B., Choi, O.R., Stevens, P.W., and Engel, J.D. (1985). Replacement variant histone genes contain intervening sequences. Mol. Cell Biol. 5, 1307-1317.

Buratowski, S. (2003). The CTD code. Nat. Struct. Biol 10, 679-680.

Buratowski, S. (2009). Progression through the RNA polymerase II CTD cycle. Mol. Cell 36, 541-546.

Cajal, S.R. (1903). Un sencillo método de coloración selectiva del retículo protoplásmico y sus efectos en los diversos órganos nerviosos de vertebrados e invertebrados. Tra. Lab. Invest. Biol. 2, 129-221. 
Cakmakci, N.G., Lerner, R.S., Wagner, E.J., Zheng, L., and Marzluff, W.F. (2008). SLIP1, a factor required for activation of histone mRNA translation by the stemloop binding protein. Mol. Cell Biol. 28, 1182-1194.

Cantin, G.T., Stevens, J.L., and Berk, A.J. (2003). Activation domain-mediator interactions promote transcription preinitiation complex assembly on promoter DNA. Proc. Natl. Acad. Sci. U. S. A 100, 12003-12008.

Cao, L., Faha, B., Dembski, M., Tsai, L.H., Harlow, E., and Dyson, N. (1992). Independent binding of the retinoblastoma protein and p107 to the transcription factor E2F. Nature 355, 176-179.

Carninci, P. (2009). Molecular biology: The long and short of RNAs. Nature 457, 974975.

Carrozza, M.J., Li, B., Florens, L., Suganuma, T., Swanson, S.K., Lee, K.K., Shia, W.J., Anderson, S., Yates, J., Washburn, M.P., and Workman, J.L. (2005). Histone H3 methylation by Set 2 directs deacetylation of coding regions by Rpd3S to suppress spurious intragenic transcription. Cell 123, 581-592.

Carvajal, D., Tovar, C., Yang, H., Vu, B.T., Heimbrook, D.C., and Vassilev, L.T. (2005). Activation of p53 by MDM2 antagonists can protect proliferating cells from mitotic inhibitors. Cancer Res. 65, 1918-1924.

Challoner, P.B., Moss, S.B., and Groudine, M. (1989). Expression of replicationdependent histone genes in avian spermatids involves an alternate pathway of mRNA 3'-end formation. Mol. Cell Biol 9, 902-913.

Chandrasekharan, M.B., Huang, F., and Sun, Z.W. (2009). Ubiquitination of histone H2B regulates chromatin dynamics by enhancing nucleosome stability. Proc. Natl. Acad. Sci. U. S. A 106, 16686-16691.

Chapman, R.D., Heidemann, M., Albert, T.K., Mailhammer, R., Flatley, A., Meisterernst, M., Kremmer, E., and Eick, D. (2007). Transcribing RNA polymerase II is phosphorylated at CTD residue serine-7. Science 318, 1780-1782.

Chaubet, N., Chaboute, M.E., Clement, B., Ehling, M., Philipps, G., and Gigot, C. (1988). The histone H3 and H4 mRNAs are polyadenylated in maize. Nucleic Acids Res. 16, 1295-1304.

Chellappan, S.P., Hiebert, S., Mudryj, M., Horowitz, J.M., and Nevins, J.R. (1991). The E2F transcription factor is a cellular target for the RB protein. Cell 65, 1053-1061.

Cheng, G.H., Nandi, A., Clerk, S., and Skoultchi, A.I. (1989). Different 3'-end processing produces two independently regulated mRNAs from a single $\mathrm{H} 1$ histone gene. Proc. Natl. Acad. Sci. U. S. A 86, 7002-7006.

Chiu, Y.L., Cao, H., Jacque, J.M., Stevenson, M., and Rana, T.M. (2004). Inhibition of human immunodeficiency virus type 1 replication by RNA interference directed against human transcription elongation factor P-TEFb (CDK9/CyclinT1). J. Virol. $78,2517-2529$. 
Cho, H., Orphanides, G., Sun, X., Yang, X.J., Ogryzko, V., Lees, E., Nakatani, Y., and Reinberg, D. (1998). A human RNA polymerase II complex containing factors that modify chromatin structure. Mol. Cell Biol 18, 5355-5363.

Choi, H.S., Choi, B.Y., Cho, Y.Y., Mizuno, H., Kang, B.S., Bode, A.M., and Dong, Z. (2005). Phosphorylation of histone $\mathrm{H} 3$ at serine 10 is indispensable for neoplastic cell transformation. Cancer Res. 65, 5818-5827.

Chu, Y., Simic, R., Warner, M.H., Arndt, K.M., and Prelich, G. (2007). Regulation of histone modification and cryptic transcription by the Bur1 and Paf1 complexes. EMBO J. 26, 4646-4656.

Chu, Y., Sutton, A., Sternglanz, R., and Prelich, G. (2006). The BUR1 cyclin-dependent protein kinase is required for the normal pattern of histone methylation by SET2. Mol. Cell Biol 26, 3029-3038.

Claudio, P.P., Cui, J., Ghafouri, M., Mariano, C., White, M.K., Safak, M., Sheffield, J.B., Giordano, A., Khalili, K., Amini, S., and Sawaya, B.E. (2006). Cdk9 phosphorylates p53 on serine 392 independently of CKII. J. Cell Physiol 208, 602612.

Cobrinik, D., Whyte, P., Peeper, D.S., Jacks, T., and Weinberg, R.A. (1993). Cell cyclespecific association of E2F with the p130 E1A-binding protein. Genes Dev. 7, 2392-2404.

Collart, D., Ramsey-Ewing, A., Bortell, R., Lian, J., Stein, J., and Stein, G. (1991). Isolation and characterization of a cDNA from a human histone $\mathrm{H} 2 \mathrm{~B}$ gene which is reciprocally expressed in relation to replication-dependent $\mathrm{H} 2 \mathrm{~B}$ histone genes during HL60 cell differentiation. Biochemistry 30, 1610-1617.

Corden, J.L. (1990). Tails of RNA polymerase II. Trends Biochem. Sci. 15, 383-387.

Corden, J.L. (2007). Transcription. Seven ups the code. Science 318, 1735-1736.

Corden, J.L., Cadena, D.L., Ahearn, J.M. Jr., and Dahmus, M.E. (1985). A unique structure at the carboxyl terminus of the largest subunit of eukaryotic RNA polymerase II. Proc. Natl. Acad. Sci. U. S. A 82, 7934-7938.

Dahmus, M.E. (1981). Phosphorylation of eukaryotic DNA-dependent RNA polymerase. Identification of calf thymus RNA polymerase subunits phosphorylated by two purified protein kinases, correlation with in vivo sites of phosphorylation in HeLa cell RNA polymerase II. J. Biol. Chem. 256, 3332-3339.

Dahmus, M.E. (1995). Phosphorylation of the C-terminal domain of RNA polymerase II. Biochim. Biophys. Acta 1261, 171-182.

Danckwardt, S., Hentze, M.W., and Kulozik, A.E. (2008). 3' end mRNA processing: molecular mechanisms and implications for health and disease. EMBO J. 27, 482498.

Dantonel, J.C., Murthy, K.G., Manley, J.L., and Tora, L. (1997). Transcription factor TFIID recruits factor CPSF for formation of 3' end of mRNA. Nature 389, 399-402. 
Das, R., Yu, J., Zhang, Z., Gygi, M.P., Krainer, A.R., Gygi, S.P., and Reed, R. (2007). SR proteins function in coupling RNAP II transcription to pre-mRNA splicing. Mol. Cell 26, 867-881.

Davie, J.R. and Murphy, L.C. (1990). Level of ubiquitinated histone H2B in chromatin is coupled to ongoing transcription. Biochemistry 29, 4752-4757.

Davila, L.M. and Samuelsson, T. (2008). Early evolution of histone mRNA 3' end processing. RNA. 14, 1-10.

De Koning, L., Corpet, A., Haber, J.E., and Almouzni, G. (2007). Histone chaperones: an escort network regulating histone traffic. Nat. Struct. Mol. Biol. 14, 997-1007.

De la Luna, S., Burden, M.J., Lee, C.W., and La Thangue, N.B. (1996). Nuclear accumulation of the E2F heterodimer regulated by subunit composition and alternative splicing of a nuclear localization signal. J. Cell Sci. 109 ( Pt 10), 24432452.

De la Mata, M., Alonso, C.R., Kadener, S., Fededa, J.P., Blaustein, M., Pelisch, F., Cramer, P., Bentley, D., and Kornblihtt, A.R. (2003). A slow RNA polymerase II affects alternative splicing in vivo. Mol. Cell 12, 525-532.

De Luca, A., Esposito, V., Baldi, A., Claudio, P.P., Fu, Y., Caputi, M., Pisano, M.M., Baldi, F., and Giordano, A. (1997). CDC2-related kinase PITALRE phosphorylates $\mathrm{pRb}$ exclusively on serine and is widely expressed in human tissues. J. Cell Physiol $172,265-273$.

DeGregori, J. and Johnson, D.G. (2006). Distinct and Overlapping Roles for E2F Family Members in Transcription, Proliferation and Apoptosis. Curr. Mol. Med. 6, 739-748.

Dehe, P.M., Pamblanco, M., Luciano, P., Lebrun, R., Moinier, D., Sendra, R., Verreault, A., Tordera, V., and Geli, V. (2005). Histone H3 lysine 4 monomethylation does not require ubiquitination of histone H2B. J. Mol. Biol. 353, 477484.

DeLeo, A.B., Jay, G., Appella, E., Dubois, G.C., Law, L.W., and Old, L.J. (1979). Detection of a transformation-related antigen in chemically induced sarcomas and other transformed cells of the mouse. Proc. Natl. Acad. Sci. U. S. A 76, 2420-2424.

DelSal, G., Loda, M., and Pagano, M. (1996). Cell cycle and cancer: critical events at the G1 restriction point. Crit Rev. Oncog. 7, 127-142.

DeRan, M., Pulvino, M., Greene, E., Su, C., and Zhao, J. (2008). Transcriptional activation of histone genes requires NPAT-dependent recruitment of TRRAPTip60 complex to histone promoters during the G1/S phase transition. Mol. Cell Biol 28, 435-447.

Ding, N., Zhou, H., Esteve, P.O., Chin, H.G., Kim, S., Xu, X., Joseph, S.M., Friez, M.J., Schwartz, C.E., Pradhan, S., and Boyer, T.G. (2008). Mediator links epigenetic silencing of neuronal gene expression with $\mathrm{x}$-linked mental retardation. Mol. Cell 31, 347-359. 
Dominski, Z. and Marzluff, W.F. (1999). Formation of the 3 ' end of histone mRNA. Gene 239, 1-14.

Dominski, Z. and Marzluff, W.F. (2007). Formation of the 3 ' end of histone mRNA: getting closer to the end. Gene 396, 373-390.

Dominski, Z., Erkmann, J.A., Yang, X., Sanchez, R., and Marzluff, W.F. (2002). A novel zinc finger protein is associated with U7 snRNP and interacts with the stemloop binding protein in the histone pre-mRNP to stimulate 3'-end processing. Genes Dev. 16, 58-71.

Dominski, Z., Yang, X.C., and Marzluff, W.F. (2005). The polyadenylation factor CPSF-73 is involved in histone-pre-mRNA processing. Cell 123, 37-48.

Donner, A.J., Ebmeier, C.C., Taatjes, D.J., and Espinosa, J.M. (2010). CDK8 is a positive regulator of transcriptional elongation within the serum response network. Nat. Struct. Mol. Biol.

Dover, J., Schneider, J., Tawiah-Boateng, M.A., Wood, A., Dean, K., Johnston, M., and Shilatifard, A. (2002). Methylation of histone H3 by COMPASS requires ubiquitination of histone H2B by Rad6. J. Biol Chem. 277, 28368-28371.

Dyson, N. (1998). The regulation of E2F by pRB-family proteins. Genes Dev. 12, 22452262.

Edmunds, J.W., Mahadevan, L.C., and Clayton, A.L. (2008). Dynamic histone H3 methylation during gene induction: HYPB/Setd2 mediates all H3K36 trimethylation. EMBO J. 27, 406-420.

Edwalds-Gilbert, G., Veraldi, K.L., and Milcarek, C. (1997). Alternative poly(A) site selection in complex transcription units: means to an end? Nucleic Acids Res. 25, 2547-2561.

Egloff, S. and Murphy, S. (2008). Cracking the RNA polymerase II CTD code. Trends Genet. 24, 280-288.

Egloff, S., Al Rawaf, H., O'Reilly, D., and Murphy, S. (2009). Chromatin structure is implicated in "late" elongation checkpoints on the U2 snRNA and beta-actin genes. Mol. Cell Biol. 29, 4002-4013.

Egloff, S., O'Reilly, D., Chapman, R.D., Taylor, A., Tanzhaus, K., Pitts, L., Eick, D., and Murphy, S. (2007). Serine-7 of the RNA polymerase II CTD is specifically required for snRNA gene expression. Science 318, 1777-1779.

Ehlers, A., Oker, E., Bentink, S., Lenze, D., Stein, H., and Hummel, M. (2008). Histone acetylation and DNA demethylation of B cells result in a Hodgkin-like phenotype. Leukemia 22, 835-841.

Eischen, C.M. and Lozano, G. (2009). p53 and MDM2: antagonists or partners in crime? Cancer Cell 15, 161-162. 
Eitoku, M., Sato, L., Senda, T., and Horikoshi, M. (2008). Histone chaperones: 30 years from isolation to elucidation of the mechanisms of nucleosome assembly and disassembly. Cell Mol. Life Sci. 65, 414-444.

El-Deiry, W.S., Kern, S.E., Pietenpol, J.A., Kinzler, K.W., and Vogelstein, B. (1992). Definition of a consensus binding site for p53. Nat. Genet. 1, 45-49.

Espinosa, J.M. (2008). Histone H2B ubiquitination: the cancer connection. Genes Dev. 22, 2743-2749.

Fahrner, K., Yarger, J., and Hereford, L. (1980). Yeast histone mRNA is polyadenylated. Nucleic Acids Res. 8, 5725-5737.

Fang, S., Jensen, J.P., Ludwig, R.L., Vousden, K.H., and Weissman, A.M. (2000). $\mathrm{Mdm} 2$ is a RING finger-dependent ubiquitin protein ligase for itself and p53. J. Biol. Chem. 275, 8945-8951.

Farber, L.J., Kort, E.J., Wang, P., Chen, J., and Teh, B.T. (2010). The tumor suppressor parafibromin is required for posttranscriptional processing of histone mRNA. Mol. Carcinog. 49, 215-223.

Fasanaro, P., Capogrossi, M.C., and Martelli, F. (2010). Regulation of the endothelial cell cycle by the ubiquitin-proteasome system. Cardiovasc. Res. 85, 272-280.

Finlay, C.A., Hinds, P.W., and Levine, A.J. (1989). The p53 proto-oncogene can act as a suppressor of transformation. Cell 57, 1083-1093.

Firestein, R., Bass, A.J., Kim, S.Y., Dunn, I.F., Silver, S.J., Guney, I., Freed, E., Ligon, A.H., Vena, N., Ogino, S., Chheda, M.G., Tamayo, P., Finn, S., Shrestha, Y., Boehm, J.S., Jain, S., Bojarski, E., Mermel, C., Barretina, J., Chan, J.A., Baselga, J., Tabernero, J., Root, D.E., Fuchs, C.S., Loda, M., Shivdasani, R.A., Meyerson, M., and Hahn, W.C. (2008). CDK8 is a colorectal cancer oncogene that regulates beta-catenin activity. Nature 455, 547-551.

Fischle, W., Wang, Y., and Allis, C.D. (2003). Histone and chromatin cross-talk. Curr. Opin. Cell Biol 15, 172-183.

Fleming, A.B., Kao, C.F., Hillyer, C., Pikaart, M., and Osley, M.A. (2008). H2B ubiquitylation plays a role in nucleosome dynamics during transcription elongation. Mol. Cell 31, 57-66.

Ford, L.P., Bagga, P.S., and Wilusz, J. (1997). The poly(A) tail inhibits the assembly of a 3'-to-5' exonuclease in an in vitro RNA stability system. Mol. Cell Biol. 17, 398406.

Freedman, D.A., Wu, L., and Levine, A.J. (1999). Functions of the MDM2 oncoprotein. Cell Mol. Life Sci. 55, 96-107.

Frey, M.R. and Matera, A.G. (1995). Coiled bodies contain U7 small nuclear RNA and associate with specific DNA sequences in interphase human cells. Proc. Natl. Acad. Sci. U. S. A 92, 5915-5919. 
Friend, K., Lovejoy, A.F., and Steitz, J.A. (2007). U2 snRNP binds intronless histone pre-mRNAs to facilitate U7-snRNP-dependent 3' end formation. Mol. Cell 28, 240252.

Fritah, A., Saucier, C., Mester, J., Redeuilh, G., and Sabbah, M. (2005). p21WAF1/CIP1 selectively controls the transcriptional activity of estrogen receptor alpha. Mol. Cell Biol. 25, 2419-2430.

Fu, J., Yoon, H.G., Qin, J., and Wong, J. (2007). Regulation of P-TEFb elongation complex activity by CDK9 acetylation. Mol. Cell Biol. 27, 4641-4651.

Fu, T.J., Peng, J., Lee, G., Price, D.H., and Flores, O. (1999). Cyclin K functions as a CDK9 regulatory subunit and participates in RNA polymerase II transcription. J. Biol Chem. 274, 34527-34530.

Fujinaga, K., Irwin, D., Huang, Y., Taube, R., Kurosu, T., and Peterlin, B.M. (2004). Dynamics of human immunodeficiency virus transcription: $\mathrm{P}-\mathrm{TEFb}$ phosphorylates $\mathrm{RD}$ and dissociates negative effectors from the transactivation response element. Mol. Cell Biol 24, 787-795.

Game, J.C. and Chernikova, S.B. (2009). The role of RAD6 in recombinational repair, checkpoints and meiosis via histone modification. DNA Repair (Amst) 8, 470-482.

Gao, G., Bracken, A.P., Burkard, K., Pasini, D., Classon, M., Attwooll, C., Sagara, M., Imai, T., Helin, K., and Zhao, J. (2003). NPAT expression is regulated by E2F and is essential for cell cycle progression. Mol. Cell Biol 23, 2821-2833.

Gaubatz, S., Lindeman, G.J., Ishida, S., Jakoi, L., Nevins, J.R., Livingston, D.M., and Rempel, R.E. (2000). E2F4 and E2F5 play an essential role in pocket proteinmediated G1 control. Mol. Cell 6, 729-735.

Geng, Y., Eaton, E.N., Picon, M., Roberts, J.M., Lundberg, A.S., Gifford, A., Sardet, C., and Weinberg, R.A. (1996). Regulation of cyclin E transcription by E2Fs and retinoblastoma protein. Oncogene 12, 1173-1180.

Ghule, P.N., Dominski, Z., Yang, X.C., Marzluff, W.F., Becker, K.A., Harper, J.W., Lian, J.B., Stein, J.L., van Wijnen, A.J., and Stein, G.S. (2008). Staged assembly of histone gene expression machinery at subnuclear foci in the abbreviated cell cycle of human embryonic stem cells. Proc. Natl. Acad. Sci. U. S. A 105, 16964-16969.

Giacinti, C. and Giordano, A. (2006). RB and cell cycle progression. Oncogene 25, 5220-5227.

Gick, O., Kramer, A., Keller, W., and Birnstiel, M.L. (1986). Generation of histone mRNA 3' ends by endonucleolytic cleavage of the pre-mRNA in a snRNPdependent in vitro reaction. EMBO J. 5, 1319-1326.

Gilmour, D.S. and Lis, J.T. (1986). RNA polymerase II interacts with the promoter region of the noninduced hsp70 gene in Drosophila melanogaster cells. Mol. Cell Biol. 6, 3984-3989. 
Glover-Cutter, K., Kim, S., Espinosa, J., and Bentley, D.L. (2008). RNA polymerase II pauses and associates with pre-mRNA processing factors at both ends of genes. Nat. Struct. Mol. Biol 15, 71-78.

Gomes, N.P., Bjerke, G., Llorente, B., Szostek, S.A., Emerson, B.M., and Espinosa, J.M. (2006). Gene-specific requirement for P-TEFb activity and RNA polymerase II phosphorylation within the p53 transcriptional program. Genes Dev. 20, 601612.

Grana, X., De Luca, A., Sang, N., Fu, Y., Claudio, P.P., Rosenblatt, J., Morgan, D.O., and Giordano, A. (1994). PITALRE, a nuclear CDC2-related protein kinase that phosphorylates the retinoblastoma protein in vitro. Proc. Natl. Acad. Sci. U. S. A 91, 3834-3838.

Guenther, M.G., Jenner, R.G., Chevalier, B., Nakamura, T., Croce, C.M., Canaani, E., and Young, R.A. (2005). Global and Hox-specific roles for the MLL1 methyltransferase. Proc. Natl. Acad. Sci. U. S. A 102, 8603-8608.

Guenther, M.G., Levine, S.S., Boyer, L.A., Jaenisch, R., and Young, R.A. (2007). A chromatin landmark and transcription initiation at most promoters in human cells. Cell 130, 77-88.

Harbour, J.W. and Dean, D.C. (2000). The Rb/E2F pathway: expanding roles and emerging paradigms. Genes Dev. 14, 2393-2409.

Hargreaves, D.C., Horng, T., and Medzhitov, R. (2009). Control of inducible gene expression by signal-dependent transcriptional elongation. Cell 138, 129-145.

He, N., Jahchan, N.S., Hong, E., Li, Q., Bayfield, M.A., Maraia, R.J., Luo, K., and Zhou, Q. (2008). A La-related protein modulates 7SK snRNP integrity to suppress P-TEFb-dependent transcriptional elongation and tumorigenesis. Mol. Cell 29, 588599.

Heintz, N., Sive, H.L., Roeder, R.G. (1983). Regulation of human histone gene expression: kinetics of accumulation and changes in the rate of synthesis and in the half-lives of individual histone mRNAs during the HeLa cell cycle. Mol. Cell Biol. 4, 539-550.

Helin, K., Harlow, E., and Fattaey, A. (1993). Inhibition of E2F-1 transactivation by direct binding of the retinoblastoma protein. Mol. Cell Biol. 13, 6501-6508.

Henikoff, S. (2000). Heterochromatin function in complex genomes. Biochim. Biophys. Acta 1470, O1-O8.

Henry, K.W., Wyce, A., Lo, W.S., Duggan, L.J., Emre, N.C., Kao, C.F., Pillus, L., Shilatifard, A., Osley, M.A., and Berger, S.L. (2003). Transcriptional activation via sequential histone H2B ubiquitylation and deubiquitylation, mediated by SAGAassociated Ubp8. Genes Dev. 17, 2648-2663.

Heo, K., Kim, H., Choi, S.H., Choi, J., Kim, K., Gu, J., Lieber, M.R., Yang, A.S., and An, W. (2008). FACT-mediated exchange of histone variant H2AX regulated by phosphorylation of H2AX and ADP-ribosylation of Spt16. Mol. Cell 30, 86-97. 
Herz,H.M., Nakanishi,S., and Shilatifard,A. (2009). The curious case of bivalent marks. Dev. Cell 17, 301-303.

Hitomi, M. and Stacey, D.W. (1999). Cyclin D1 production in cycling cells depends on ras in a cell-cycle-specific manner. Curr. Biol. 9, 1075-1084.

Ho, C.K. and Shuman, S. (1999). Distinct roles for CTD Ser-2 and Ser-5 phosphorylation in the recruitment and allosteric activation of mammalian mRNA capping enzyme. Mol. Cell 3, 405-411.

Hobom, U. and Dobbelstein, M. (2004). E1B-55-kilodalton protein is not required to block p53-induced transcription during adenovirus infection. J. Virol. 78, 76857697.

Hochegger, H., Takeda, S., and Hunt, T. (2008). Cyclin-dependent kinases and cellcycle transitions: does one fit all? Nat. Rev. Mol. Cell Biol. 9, 910-916.

Hochstrasser, M. (1996). Protein degradation or regulation: Ub the judge. Cell 84, 813815.

Hollstein, M., Sidransky, D., Vogelstein, B., and Harris, C.C. (1991). p53 mutations in human cancers. Science 253, 49-53.

Huang, Y., Fang, J., Bedford, M.T., Zhang, Y., and Xu, R.M. (2006). Recognition of histone $\mathrm{H} 3$ lysine-4 methylation by the double tudor domain of JMJD2A. Science $312,748-751$.

Huen, M.S., Grant, R., Manke, I., Minn, K., Yu, X., Yaffe, M.B., and Chen, J. (2007). RNF8 transduces the DNA-damage signal via histone ubiquitylation and checkpoint protein assembly. Cell 131, 901-914.

Huet, X., Rech, J., Plet, A., Vie, A., and Blanchard, J.M. (1996). Cyclin A expression is under negative transcriptional control during the cell cycle. Mol. Cell Biol. 16, 3789-3798.

Hwang, W.W., Venkatasubrahmanyam, S., Ianculescu, A.G., Tong, A., Boone, C., and Madhani, H.D. (2003). A conserved RING finger protein required for histone H2B monoubiquitination and cell size control. Mol. Cell 11, 261-266.

Iankova, I., Petersen, R.K., Annicotte, J.S., Chavey, C., Hansen, J.B., Kratchmarova, I., Sarruf, D., Benkirane, M., Kristiansen, K., and Fajas, L. (2006). Peroxisome proliferator-activated receptor gamma recruits the positive transcription elongation factor $\mathrm{b}$ complex to activate transcription and promote adipogenesis. Mol. Endocrinol. 20, 1494-1505.

Imai, T., Yamauchi, M., Seki, N., Sugawara, T., Saito, T., Matsuda, Y., Ito, H., Nagase, T., Nomura, N., and Hori, T. (1996). Identification and characterization of a new gene physically linked to the ATM gene. Genome Res. 6, 439-447.

Imai, Y., Kimura, T., Murakami, A., Yajima, N., Sakamaki, K., and Yonehara, S. (1999). The CED-4-homologous protein FLASH is involved in Fas-mediated activation of caspase- 8 during apoptosis. Nature $398,777-785$. 
Ivanov, D., Kwak, Y.T., Guo, J., and Gaynor, R.B. (2000). Domains in the SPT5 protein that modulate its transcriptional regulatory properties. Mol. Cell Biol 20, 2970-2983.

Iwakuma, T. and Lozano, G. (2003). MDM2, an introduction. Mol. Cancer Res. 1, 9931000.

Izaurralde, E., Lewis, J., McGuigan, C., Jankowska, M., Darzynkiewicz, E., and Mattaj, I.W. (1994). A nuclear cap binding protein complex involved in pre-mRNA splicing. Cell 78, 657-668.

Jang, M.K., Mochizuki, K., Zhou, M., Jeong, H.S., Brady, J.N., and Ozato, K. (2005). The bromodomain protein $\mathrm{Brd} 4$ is a positive regulatory component of $\mathrm{P}-\mathrm{TEFb}$ and stimulates RNA polymerase II-dependent transcription. Mol. Cell 19, 523-534.

Jenuwein, T. and Allis, C.D. (2001). Translating the histone code. Science 293, 10741080.

Johnsen, S.A., Güngör, C., Prenzel, T., Riethdorf, S., Riethdorf, L., Taniguchi-Ishigaki, N., Rau, T., Tursun, B., Furlow, J.D., Sauter, G., Pantel, K., Gannon, F., and Bach, I. (2009). Regulation of estrogen-dependent transcription by the LIM cofactors CLIM and RLIM in breast cancer. Cancer Res. 69, 128-136.

Johnsen, S.A., Subramaniam, M., Monroe, D.G., Janknecht, R., and Spelsberg, T.C. (2002). Modulation of transforming growth factor beta (TGFbeta)/Smad transcriptional responses through targeted degradation of TGFbeta-inducible early gene-1 by human seven in absentia homologue. J. Biol. Chem. 277, 30754-30759.

Johnson, D.G. and Schneider-Broussard, R. (1998). Role of E2F in cell cycle control and cancer. Front Biosci. 3, d447-d448.

Kaygun, H. and Marzluff, W.F. (2005). Regulated degradation of replication-dependent histone mRNAs requires both ATR and Upf1. Nat. Struct. Mol. Biol. 12, 794-800.

Keogh, M.C., Podolny, V., and Buratowski, S. (2003). Bur1 kinase is required for efficient transcription elongation by RNA polymerase II. Mol. Cell Biol 23, 70057018.

Kim, J., Guermah, M., McGinty, R.K., Lee, J.S., Tang, Z., Milne, T.A., Shilatifard, A., Muir, T.W., and Roeder, R.G. (2009). RAD6-Mediated transcription-coupled H2B ubiquitylation directly stimulates H3K4 methylation in human cells. Cell 137, 459471.

Kim, J., Hake, S.B., and Roeder, R.G. (2005a). The human homolog of yeast BRE1 functions as a transcriptional coactivator through direct activator interactions. Mol. Cell 20, 759-770.

Kim, J.B. and Sharp, P.A. (2001). Positive transcription elongation factor B phosphorylates hSPT5 and RNA polymerase II carboxyl-terminal domain independently of cyclin-dependent kinase-activating kinase. J. Biol Chem. 276, 12317-12323. 
Kim, M., Krogan, N.J., Vasiljeva, L., Rando, O.J., Nedea, E., Greenblatt, J.F., and Buratowski, S. (2004). The yeast Rat1 exonuclease promotes transcription termination by RNA polymerase II. Nature 432, 517-522.

Kim, T.H., Barrera, L.O., Zheng, M., Qu, C., Singer, M.A., Richmond, T.A., Wu, Y., Green, R.D., and Ren, B. (2005b). A high-resolution map of active promoters in the human genome. Nature 436, 876-880.

Kirsh, A.L., Groudine, M., and Challoner, P.B. (1989). Polyadenylation and U7 snRNPmediated cleavage: alternative modes of RNA 3' processing in two avian histone H1 genes. Genes Dev. 3, 2172-2179.

Kizer, K.O., Phatnani, H.P., Shibata, Y., Hall, H., Greenleaf, A.L., and Strahl, B.D. (2005). A novel domain in Set 2 mediates RNA polymerase II interaction and couples histone H3 K36 methylation with transcript elongation. Mol. Cell Biol 25, 3305-3316.

Knuesel, M.T., Meyer, K.D., Bernecky, C., and Taatjes, D.J. (2009). The human CDK8 subcomplex is a molecular switch that controls Mediator coactivator function. Genes Dev. 23, 439-451.

Kobayashi, H., Stewart, E., Poon, R.Y., and Hunt, T. (1994). Cyclin A and cyclin B dissociate from p34cdc2 with half-times of 4 and $15 \mathrm{~h}$, respectively, regardless of the phase of the cell cycle. J. Biol. Chem. 269, 29153-29160.

Koken, M.H., Reynolds, P., Jaspers-Dekker, I., Prakash, L., Prakash, S., Bootsma, D., and Hoeijmakers, J.H. (1991). Structural and functional conservation of two human homologs of the yeast DNA repair gene RAD6. Proc. Natl. Acad. Sci. U. S. A 88, 8865-8869.

Kolasinska-Zwierz, P., Down, T., Latorre, I., Liu, T., Liu, X.S., and Ahringer, J. (2009). Differential chromatin marking of introns and expressed exons by H3K36me3. Nat. Genet. 41, 376-381.

Kolev, N.G. and Steitz, J.A. (2005). Symplekin and multiple other polyadenylation factors participate in 3'-end maturation of histone mRNAs. Genes Dev. 19, 25832592.

Komarnitsky, P., Cho, E.J., and Buratowski, S. (2000). Different phosphorylated forms of RNA polymerase II and associated mRNA processing factors during transcription. Genes Dev. 14, 2452-2460.

Konev, A.Y., Tribus, M., Park, S.Y., Podhraski, V., Lim, C.Y., Emelyanov, A.V., Vershilova, E., Pirrotta, V., Kadonaga, J.T., Lusser, A., and Fyodorov, D.V. (2007). CHD1 motor protein is required for deposition of histone variant H3.3 into chromatin in vivo. Science 317, 1087-1090.

Koonin, E.V., Aravind, L., Hofmann, K., Tschopp, J., and Dixit, V.M. (1999). Apoptosis. Searching for FLASH domains. Nature 401, 662-663.

Kornberg, R.D. (1974). Chromatin structure: a repeating unit of histones and DNA. Science $184,868-871$. 
Kornberg, R.D. (1977). Structure of chromatin. Annu. Rev. Biochem. 46, 931-954.

Kranz, D. and Dobbelstein, M. (2006). Nongenotoxic p53 activation protects cells against S-phase-specific chemotherapy. Cancer Res. 66, 10274-10280.

Kranz, D., Dohmesen, C., and Dobbelstein, M. (2008). BRCA1 and Tip60 determine the cellular response to ultraviolet irradiation through distinct pathways. J. Cell Biol 182, 197-213.

Krek, W., Livingston, D.M., and Shirodkar, S. (1993). Binding to DNA and the retinoblastoma gene product promoted by complex formation of different E2F family members. Science 262, 1557-1560.

Krivtsov, A.V., Feng, Z., Lemieux, M.E., Faber, J., Vempati, S., Sinha, A.U., Xia, X., Jesneck, J., Bracken, A.P., Silverman, L.B., Kutok, J.L., Kung, A.L., and Armstrong, S.A. (2008). H3K79 methylation profiles define murine and human MLL-AF4 leukemias. Cancer Cell 14, 355-368.

Krogan, N.J., Kim, M., Ahn, S.H., Zhong, G., Kobor, M.S., Cagney, G., Emili, A., Shilatifard, A., Buratowski, S., and Greenblatt, J.F. (2002). RNA polymerase II elongation factors of Saccharomyces cerevisiae: a targeted proteomics approach. Mol. Cell Biol. 22, 6979-6992.

Krogan, N.J., Kim, M., Tong, A., Golshani, A., Cagney, G., Canadien, V., Richards, D.P., Beattie, B.K., Emili, A., Boone, C., Shilatifard, A., Buratowski, S., and Greenblatt, J. (2003). Methylation of histone H3 by Set2 in Saccharomyces cerevisiae is linked to transcriptional elongation by RNA polymerase II. Mol. Cell Biol 23, 4207-4218.

Krueger, B.J., Jeronimo, C., Roy, B.B., Bouchard, A., Barrandon, C., Byers, S.A., Searcey, C.E., Cooper, J.J., Bensaude, O., Cohen, E.A., Coulombe, B., and Price, D.H. (2008). LARP7 is a stable component of the 7SK snRNP while P-TEFb, HEXIM1 and hnRNP A1 are reversibly associated. Nucleic Acids Res. 36, 22192229.

Kuras, L. and Struhl, K. (1999). Binding of TBP to promoters in vivo is stimulated by activators and requires Pol II holoenzyme. Nature 399, 609-613.

Kussie, P.H., Gorina, S., Marechal, V., Elenbaas, B., Moreau, J., Levine, A.J., and Pavletich, N.P. (1996). Structure of the MDM2 oncoprotein bound to the p53 tumor suppressor transactivation domain. Science 274, 948-953.

Lane, D.P. and Crawford, L.V. (1979). T antigen is bound to a host protein in SV40transformed cells. Nature 278, 261-263.

Laribee, R.N., Fuchs, S.M., and Strahl, B.D. (2007). H2B ubiquitylation in transcriptional control: a FACT-finding mission. Genes Dev. 21, 737-743.

Laribee, R.N., Krogan, N.J., Xiao, T., Shibata, Y., Hughes, T.R., Greenblatt, J.F., and Strahl, B.D. (2005). BUR kinase selectively regulates H3 K4 trimethylation and $\mathrm{H} 2 \mathrm{~B}$ ubiquitylation through recruitment of the PAF elongation complex. Curr. Biol $15,1487-1493$. 
Lee, T.I. and Young, R.A. (2000). Transcription of eukaryotic protein-coding genes. Annu. Rev. Genet. 34, 77-137.

Lee, T.I., Jenner, R.G., Boyer, L.A., Guenther, M.G., Levine, S.S., Kumar, R.M., Chevalier, B., Johnstone, S.E., Cole, M.F., Isono, K., Koseki, H., Fuchikami, T., Abe, K., Murray, H.L., Zucker, J.P., Yuan, B., Bell, G.W., Herbolsheimer, E., Hannett, N.M., Sun, K., Odom, D.T., Otte, A.P., Volkert, T.L., Bartel, D.P., Melton, D.A., Gifford, D.K., Jaenisch, R., and Young, R.A. (2006). Control of developmental regulators by Polycomb in human embryonic stem cells. Cell 125, 301-313.

Lee, W.H., Bookstein, R., Hong, F., Young, L.J., Shew, J.Y., and Lee, E.Y. (1987). Human retinoblastoma susceptibility gene: cloning, identification, and sequence. Science 235, 1394-1399.

Lemm, I., Girard, C., Kuhn, A.N., Watkins, N.J., Schneider, M., Bordonné, R., and Lührmann, R. (2006). Ongoing U snRNP biogenesis is required for the integrity of Cajal bodies. Mol. Biol Cell 17, 3221-3231.

Levchenko, V., Jackson, B., and Jackson, V. (2005). Histone release during transcription: displacement of the two $\mathrm{H} 2 \mathrm{~A}-\mathrm{H} 2 \mathrm{~B}$ dimers in the nucleosome is dependent on different levels of transcription-induced positive stress. Biochemistry $44,5357-5372$.

Li, B., Carey, M., and Workman, J.L. (2007). The role of chromatin during transcription. Cell 128, 707-719.

Li, B., Howe, L., Anderson, S., Yates, J.R. III, and Workman, J.L. (2003). The Set2 histone methyltransferase functions through the phosphorylated carboxyl-terminal domain of RNA polymerase II. J. Biol Chem. 278, 8897-8903.

Li, M., Phatnani, H.P., Guan, Z., Sage, H., Greenleaf, A.L., and Zhou, P. (2005a). Solution structure of the Set2-Rpb1 interacting domain of human Set2 and its interaction with the hyperphosphorylated C-terminal domain of Rpb1. Proc. Natl. Acad. Sci. U. S. A 102, 17636-17641.

Li, Q., Price, J.P., Byers, S.A., Cheng, D., Peng, J., and Price, D.H. (2005b). Analysis of the large inactive P-TEFb complex indicates that it contains one 7SK molecule, a dimer of HEXIM1 or HEXIM2, and two P-TEFb molecules containing Cdk9 phosphorylated at threonine 186. J. Biol. Chem. 280, 28819-28826.

Li, X.Y., Virbasius, A., Zhu, X., and Green, M.R. (1999). Enhancement of TBP binding by activators and general transcription factors. Nature 399, 605-609.

Licatalosi, D.D., Geiger, G., Minet, M., Schroeder, S., Cilli, K., McNeil, J.B., and Bentley, D.L. (2002). Functional interaction of yeast pre-mRNA 3' end processing factors with RNA polymerase II. Mol. Cell 9, 1101-1111.

Linzer, D.I. and Levine, A.J. (1979). Characterization of a 54K dalton cellular SV40 tumor antigen present in SV40-transformed cells and uninfected embryonal carcinoma cells. Cell 17, 43-52. 
Listerman, I., Sapra, A.K., and Neugebauer, K.M. (2006). Cotranscriptional coupling of splicing factor recruitment and precursor messenger RNA splicing in mammalian cells. Nat. Struct. Mol. Biol 13, 815-822.

Liu, J.L., Murphy, C., Buszczak, M., Clatterbuck, S., Goodman, R., and Gall, J.G. (2006). The Drosophila melanogaster Cajal body. J. Cell Biol. 172, 875-884.

Lohr, K., Moritz, C., Contente, A., and Dobbelstein, M. (2003). p21/CDKN1A mediates negative regulation of transcription by p53. J. Biol Chem. 278, 32507-32516.

Luger, K., Mader, A.W., Richmond, R.K., Sargent, D.F., and Richmond, T.J. (1997). Crystal structure of the nucleosome core particle at 2.8 A resolution. Nature 389, 251-260.

Lundberg, A.S. and Weinberg, R.A. (1998). Functional inactivation of the retinoblastoma protein requires sequential modification by at least two distinct cyclin-cdk complexes. Mol. Cell Biol. 18, 753-761.

Ma, T., Van Tine, B.A., Wei, Y., Garrett, M.D., Nelson, D., Adams, P.D., Wang, J., Qin, J., Chow, L.T., and Harper, J.W. (2000). Cell cycle-regulated phosphorylation of p220(NPAT) by cyclin E/Cdk2 in Cajal bodies promotes histone gene transcription. Genes Dev. 14, 2298-2313.

Ma, Y., Chen, L., Wright, G.M., Pillai, S.R., Chellappan, S.P., and Cress, W.D. (2010). CDKN1C negatively regulates RNA polymerase II C-terminal domain phosphorylation in an E2F1-dependent manner. J. Biol. Chem.

Mailand, N., Bekker-Jensen, S., Faustrup, H., Melander, F., Bartek, J., Lukas, C., and Lukas, J. (2007). RNF8 ubiquitylates histones at DNA double-strand breaks and promotes assembly of repair proteins. Cell 131, 887-900.

Malumbres, M. and Barbacid, M. (2005). Mammalian cyclin-dependent kinases. Trends Biochem. Sci. 30, 630-641.

Mandal, S.S., Chu, C., Wada, T., Handa, H., Shatkin, A.J., and Reinberg, D. (2004). Functional interactions of RNA-capping enzyme with factors that positively and negatively regulate promoter escape by RNA polymerase II. Proc. Natl. Acad. Sci. U. S. A 101, 7572-7577.

Manley, J.L. (2002). Nuclear coupling: RNA processing reaches back to transcription. Nat. Struct. Biol. 9, 790-791.

Margaritis, T. and Holstege, F.C. (2008). Poised RNA polymerase II gives pause for thought. Cell 133, 581-584.

Markert, A., Grimm, M., Martinez, J., Wiesner, J., Meyerhans, A., Meyuhas, O., Sickmann, A., and Fischer, U. (2008). The La-related protein LARP7 is a component of the 7SK ribonucleoprotein and affects transcription of cellular and viral polymerase II genes. EMBO Rep. 9, 569-575.

Marshall, N.F. and Price, D.H. (1992). Control of formation of two distinct classes of RNA polymerase II elongation complexes. Mol. Cell Biol 12, 2078-2090. 
Marshall, N.F., Peng, J., Xie, Z., and Price, D.H. (1996). Control of RNA polymerase II elongation potential by a novel carboxyl-terminal domain kinase. J. Biol Chem. $271,27176-27183$.

Marshall, R.M. and Grana, X. (2006). Mechanisms controlling CDK9 activity. Front Biosci. 11, 2598-2613.

Martinez, I., Wang, J., Hobson, K.F., Ferris, R.L., and Khan, S.A. (2007). Identification of differentially expressed genes in HPV-positive and HPV-negative oropharyngeal squamous cell carcinomas. Eur. J. Cancer 43, 415-432.

Marzluff, W.F. (1992). Histone 3' ends: essential and regulatory functions. Gene Expr. 2, 93-97.

Marzluff, W.F., Gongidi, P., Woods, K.R., Jin, J., and Maltais, L.J. (2002). The human and mouse replication-dependent histone genes. Genomics 80, 487-498.

Marzluff, W.F., Wagner, E.J., and Duronio, R.J. (2008). Metabolism and regulation of canonical histone mRNAs: life without a poly(A) tail. Nat. Rev. Genet. 9, 843-854.

Mason, P.B. and Struhl, K. (2003). The FACT complex travels with elongating RNA polymerase II and is important for the fidelity of transcriptional initiation in vivo. Mol. Cell Biol. 23, 8323-8333.

Matera, A.G. (2006). Drosophila Cajal bodies: accessories not included. J. Cell Biol. 172, 791-793.

Mayr, C. and Bartel, D.P. (2009). Widespread Shortening of 3'UTRs by Alternative Cleavage and Polyadenylation Activates Oncogenes in Cancer Cells. Cell 138, 673684.

McCracken, S., Fong, N., Rosonina, E., Yankulov, K., Brothers, G., Siderovski, D., Hessel, A., Foster, S., Shuman, S., and Bentley, D.L. (1997). 5'-Capping enzymes are targeted to pre-mRNA by binding to the phosphorylated carboxy-terminal domain of RNA polymerase II. Genes Dev. 11, 3306-3318.

McGinty, R.K., Kim, J., Chatterjee, C., Roeder, R.G., and Muir, T.W. (2008). Chemically ubiquitylated histone H2B stimulates hDot1L-mediated intranucleosomal methylation. Nature 453, 812-816.

Medlin, J., Scurry, A., Taylor, A., Zhang, F., Peterlin, B.M., and Murphy, S. (2005). P$\mathrm{TEFb}$ is not an essential elongation factor for the intronless human U2 snRNA and histone H2b genes. EMBO J. 24, 4154-4165.

Meinhart, A. and Cramer, P. (2004). Recognition of RNA polymerase II carboxyterminal domain by 3'-RNA-processing factors. Nature 430, 223-226.

Meinhart, A., Kamenski, T., Hoeppner, S., Baumli, S., and Cramer, P. (2005). A structural perspective of CTD function. Genes Dev. 19, 1401-1415.

Michels, A.A., Fraldi, A., Li, Q., Adamson, T.E., Bonnet, F., Nguyen, V.T., Sedore, S.C., Price, J.P., Price ,D.H., Lania ,L., and Bensaude ,O. (2004). Binding of the 
7SK snRNA turns the HEXIM1 protein into a P-TEFb (CDK9/cyclin T) inhibitor. EMBO J. 23, 2608-2619.

Michels, A.A., Nguyen, V.T., Fraldi, A., Labas, V., Edwards, M., Bonnet, F., Lania, L., and Bensaude, O. (2003). MAQ1 and 7SK RNA interact with CDK9/cyclin T complexes in a transcription-dependent manner. Mol. Cell Biol. 23, 4859-4869.

Mikkelsen, T.S., Ku, M., Jaffe, D.B., Issac, B., Lieberman, E., Giannoukos, G., Alvarez, P., Brockman, W., Kim, T.K., Koche, R.P., Lee, W., Mendenhall, E., O'Donovan, A., Presser, A., Russ, C., Xie, X., Meissner, A., Wernig, M., Jaenisch, R., Nusbaum, C., Lander, E.S., and Bernstein, B.E. (2007). Genome-wide maps of chromatin state in pluripotent and lineage-committed cells. Nature 448, 553-560.

Minsky, N., Shema, E., Field, Y., Schuster, M., Segal, E., and Oren, M. (2008). Monoubiquitinated $\mathrm{H} 2 \mathrm{~B}$ is associated with the transcribed region of highly expressed genes in human cells. Nat. Cell Biol 10, 483-488.

Mitchison, J.M. (1971). The Biology of the Cell Cycle (Cambridge: Cambridge University Press).

Mitra, P., Ghule, P.N., van der Deen, M., Medina, R., Xie, R.L., Holmes, W.F., Ye, X., Nakayama, K.I., Harper, J.W., Stein, J.L., Stein, G.S., and van Wijnen, A.J. (2009). CDK inhibitors selectively diminish cell cycle controlled activation of the histone H4 gene promoter by p220NPAT and HiNF-P. J. Cell Physiol 219, 438-448.

Mitra, P., Xie, R.L., Medina, R., Hovhannisyan, H., Zaidi, S.K., Wei, Y., Harper, J.W., Stein, J.L., van Wijnen, A.J., and Stein, G.S. (2003). Identification of HiNF-P, a key activator of cell cycle-controlled histone $\mathrm{H} 4$ genes at the onset of S phase. Mol. Cell Biol. 23, 8110-8123.

Morgan, D.O. (1995). Principles of CDK regulation. Nature 374, 131-134.

Moss, S.B., Ferry, R.A., and Groudine, M. (1994). An alternative pathway of histone mRNA 3' end formation in mouse round spermatids. Nucleic Acids Res. 22, 31603166.

Mowry, K.L. and Steitz, J.A. (1987). Identification of the human U7 snRNP as one of several factors involved in the 3' end maturation of histone premessenger RNA's. Science 238, 1682-1687.

Mueller, C.L., Porter, S.E., Hoffman, M.G., and Jaehning, J.A. (2004). The Paf1 complex has functions independent of actively transcribing RNA polymerase II. Mol. Cell 14, 447-456.

Mueller, R.D., Yasuda, H., Hatch, C.L., Bonner, W.M., and Bradbury, E.M. (1985). Identification of ubiquitinated histones $2 \mathrm{~A}$ and $2 \mathrm{~B}$ in Physarum polycephalum. Disappearance of these proteins at metaphase and reappearance at anaphase. J. Biol. Chem. 260, 5147-5153.

Muse, G.W., Gilchrist, D.A., Nechaev, S., Shah, R., Parker, J.S., Grissom, S.F., Zeitlinger, J., and Adelman, K. (2007). RNA polymerase is poised for activation across the genome. Nat. Genet. 39, 1507-1511. 
Nakayama, K. and Nakayama, K. (1998). Cip/Kip cyclin-dependent kinase inhibitors: brakes of the cell cycle engine during development. Bioessays 20, 1020-1029.

Narita, T., Yamaguchi, Y., Yano, K., Sugimoto, S., Chanarat, S., Wada, T., Kim, D.K., Hasegawa, J., Omori, M., Inukai, N., Endoh, M., Yamada, T., and Handa, H. (2003). Human transcription elongation factor NELF: identification of novel subunits and reconstitution of the functionally active complex. Mol. Cell Biol. 23, 1863-1873.

Narita, T., Yung, T.M., Yamamoto, J., Tsuboi, Y., Tanabe, H., Tanaka, K., Yamaguchi, Y., and Handa, H. (2007). NELF interacts with CBC and participates in 3' end processing of replication-dependent histone mRNAs. Mol. Cell 26, 349-365.

Natalizio, B.J., Robson-Dixon, N.D., and Garcia-Blanco, M.A. (2009). The Carboxylterminal Domain of RNA Polymerase II Is Not Sufficient to Enhance the Efficiency of Pre-mRNA Capping or Splicing in the Context of a Different Polymerase. J. Biol. Chem. 284, 8692-8702.

Nelson, J.D., Denisenko, O., and Bomsztyk, K. (2006). Protocol for the fast chromatin immunoprecipitation (ChIP) method. Nat. Protoc. 1, 179-185.

Ng, H.H., Robert, F., Young, R.A., and Struhl, K. (2003). Targeted recruitment of Set1 histone methylase by elongating Pol II provides a localized mark and memory of recent transcriptional activity. Mol. Cell 11, 709-719.

Ng, H.H., Xu, R.M., Zhang, Y., and Struhl, K. (2002). Ubiquitination of histone H2B by Rad6 is required for efficient Dot1-mediated methylation of histone H3 lysine 79. J. Biol. Chem. 277, 34655-34657.

Nguyen, V.T., Kiss, T., Michels, A.A., and Bensaude, O. (2001). 7SK small nuclear RNA binds to and inhibits the activity of CDK9/cyclin T complexes. Nature 414, 322-325.

Nigg, E.A. (2001). Cell cycle regulation by protein kinases and phosphatases. Ernst. Schering. Res. Found. Workshop 19-46.

Nilsson, I. and Hoffmann, I. (2000). Cell cycle regulation by the Cdc25 phosphatase family. Prog. Cell Cycle Res. 4, 107-114.

Norbury, C. and Nurse, P. (1992). Animal cell cycles and their control. Annu. Rev. Biochem. 61, 441-470.

Nurse, P. (2000). A long twentieth century of the cell cycle and beyond. Cell 100, 7178.

Ogris, E., Rotheneder, H., Mudrak, I., Pichler,A. , and Wintersberger, E. (1993). A binding site for transcription factor E2F is a target for trans activation of murine thymidine kinase by polyomavirus large $\mathrm{T}$ antigen and plays an important role in growth regulation of the gene. J. Virol. 67, 1765-1771.

Ohtani, K., DeGregori, J., and Nevins, J.R. (1995). Regulation of the cyclin E gene by transcription factor E2F1. Proc. Natl. Acad. Sci. U. S. A 92, 12146-12150. 
Onel, K. and Cordon-Cardo, C. (2004). MDM2 and prognosis. Mol. Cancer Res. 2, 1-8.

Ormondroyd, E., de la Luna, S., and La Thangue, N.B. (1995). A new member of the DP family, DP-3, with distinct protein products suggests a regulatory role for alternative splicing in the cell cycle transcription factor DRTF1/E2F. Oncogene 11, 1437-1446.

Orphanides, G., Lagrange, T., and Reinberg, D. (1996). The general transcription factors of RNA polymerase II. Genes Dev. 10, 2657-2683.

Orphanides, G., LeRoy, G., Chang, C.H., Luse, D.S., and Reinberg, D. (1998). FACT, a factor that facilitates transcript elongation through nucleosomes. Cell 92, 105-116.

Orphanides, G., Wu, W.H., Lane, W.S., Hampsey, M., and Reinberg, D. (1999). The chromatin-specific transcription elongation factor FACT comprises human SPT16 and SSRP1 proteins. Nature 400, 284-288.

Osley, M.A. (1991). The regulation of histone synthesis in the cell cycle. Annu. Rev. Biochem. 60, 827-861.

Osley, M.A. (2004). H2B ubiquitylation: the end is in sight. Biochim. Biophys. Acta 1677, 74-78.

Osley, M.A. (2006). Regulation of histone H2A and H2B ubiquitylation. Brief. Funct. Genomic. Proteomic. 5, 179-189.

Pal, M. and Luse, D.S. (2003). The initiation-elongation transition: lateral mobility of RNA in RNA polymerase II complexes is greatly reduced at $+8 /+9$ and absent by +23. Proc. Natl. Acad. Sci. U. S. A 100, 5700-5705.

Pandey, N.B. and Marzluff, W.F. (1987). The stem-loop structure at the 3' end of histone mRNA is necessary and sufficient for regulation of histone mRNA stability. Mol. Cell Biol. 7, 4557-4559.

Pandey, N.B., Chodchoy, N., Liu, T.J., and Marzluff, W.F. (1990). Introns in histone genes alter the distribution of 3' ends. Nucleic Acids Res. 18, 3161-3170.

Parker, R. and Song, H. (2004). The enzymes and control of eukaryotic mRNA turnover. Nat. Struct. Mol. Biol. 11, 121-127.

Pavri, R., Zhu, B., Li, G., Trojer, P., Mandal, S., Shilatifard, A., and Reinberg, D. (2006). Histone H2B monoubiquitination functions cooperatively with FACT to regulate elongation by RNA polymerase II. Cell 125, 703-717.

Pearson, B.E., Nasheuer, H.P., and Wang, T.S. (1991). Human DNA polymerase alpha gene: sequences controlling expression in cycling and serum-stimulated cells. Mol. Cell Biol. 11, 2081-2095.

Peng, J., Marshall, N.F., and Price, D.H. (1998a). Identification of a cyclin subunit required for the function of Drosophila P-TEFb. J. Biol. Chem. 273, 13855-13860.

Peng, J., Zhu, Y., Milton, J.T., and Price, D.H. (1998b). Identification of multiple cyclin subunits of human P-TEFb. Genes Dev. 12, 755-762. 
Perales, R. and Bentley, D. (2009). "Cotranscriptionality": the transcription elongation complex as a nexus for nuclear transactions. Mol. Cell 36, 178-191.

Peterlin, B.M. and Price, D.H. (2006). Controlling the elongation phase of transcription with P-TEFb. Mol. Cell 23, 297-305.

Peterson, C.L. and Laniel, M.A. (2004). Histones and histone modifications. Curr. Biol. 14, R546-R551.

Phatnani, H.P. and Greenleaf, A.L. (2006). Phosphorylation and functions of the RNA polymerase II CTD. Genes Dev. 20, 2922-2936.

Pickart, C.M. (2001). Ubiquitin enters the new millennium. Mol. Cell 8, 499-504.

Pillai, R.S., Grimmler, M., Meister, G., Will, C.L., Lührmann, R., Fischer,U., and Schümperli, D. (2003). Unique Sm core structure of U7 snRNPs: assembly by a specialized SMN complex and the role of a new component, Lsm11, in histone RNA processing. Genes Dev. 17, 2321-2333.

Ping, Y.H., Chu, C.Y., Cao, H., Jacque, J.M., Stevenson, M., and Rana, T.M. (2004). Modulating HIV-1 replication by RNA interference directed against human transcription elongation factor SPT5. Retrovirology. 1, 46.

Pirngruber, J., Shchebet, A., and Johnsen, S.A. (2009a). Insights into the function of the human $\mathrm{P}-\mathrm{TEFb}$ component CDK9 in the regulation of chromatin modifications and co-transcriptional mRNA processing. Cell Cycle 8, 3636-3642.

Pirngruber, J., Shchebet, A., Schreiber, L., Shema, E., Minsky, N., Chapman, R.D., Eick, D., Aylon, Y., Oren, M., and Johnsen, S.A. (2009b). CDK9 directs H2B monoubiquitination and controls replication-dependent histone mRNA 3' end processing. EMBO Rep. 10, 894-900.

Pokholok, D.K., Harbison, C.T., Levine, S., Cole, M., Hannett, N.M., Lee, T.I., Bell, G.W., Walker, K., Rolfe, P.A., Herbolsheimer, E., Zeitlinger, J., Lewitter, F., Gifford, D.K., and Young, R.A. (2005). Genome-wide map of nucleosome acetylation and methylation in yeast. Cell 122, 517-527.

Polager, S. and Ginsberg, D. (2009). p53 and E2f: partners in life and death. Nat. Rev. Cancer 9, 738-748.

Poznic, M. (2009). Retinoblastoma protein: a central processing unit. J. Biosci. 34, 305312.

Pray-Grant, M.G., Daniel, J.A., Schieltz, D., Yates, J.R. III, and Grant, P.A. (2005). Chd1 chromodomain links histone H3 methylation with. Nature 433, 434-438.

Preiss, T. and Hentze, M.W. (1998). Dual function of the messenger RNA cap structure in poly(A)-tail-promoted translation in yeast. Nature 392, 516-520.

Preker, P., Nielsen, J., Kammler, S., Lykke-Andersen, S., Christensen, M.S., Mapendano, C.K., Schierup, M.H., and Jensen, T.H. (2008). RNA exosome depletion reveals transcription upstream of active human promoters. Science 322, 1851-1854. 
Price, D.H. (2008). Poised polymerases: on your mark...get set...go! Mol. Cell 30, 7-10.

Proudfoot, N. (2004). New perspectives on connecting messenger RNA 3' end formation to transcription. Curr. Opin. Cell Biol. 16, 272-278.

Proudfoot,N.J., Furger,A., and Dye,M.J. (2002). Integrating mRNA processing with transcription. Cell 108, 501-512.

Radhakrishnan, S.K. and Gartel, A.L. (2006). CDK9 phosphorylates p53 on serine residues 33, 315 and 392. Cell Cycle 5, 519-521.

Rappold, I., Iwabuchi, K., Date, T., and Chen, J. (2001). Tumor suppressor p53 binding protein 1 (53BP1) is involved in DNA damage-signaling pathways. J. Cell Biol $153,613-620$.

Rayasam, G.V., Wendling, O., Angrand, P.O., Mark, M., Niederreither, K., Song, L., Lerouge, T., Hager, G.L., Chambon, P., and Losson, R. (2003). NSD1 is essential for early post-implantation development and has a catalytically active SET domain. EMBO J. 22, 3153-3163.

Reinberg, D. and Sims, R.J. III (2006). de FACTo nucleosome dynamics. J. Biol. Chem. 281, 23297-23301.

Renner, D.B., Yamaguchi, Y., Wada, T., Handa, H., and Price, D.H. (2001). A highly purified RNA polymerase II elongation control system. J. Biol. Chem. 276, 4260142609.

Richards, E.J. and Elgin, S.C. (2002). Epigenetic codes for heterochromatin formation and silencing: rounding up the usual suspects. Cell 108, 489-500.

Robzyk, K., Recht, J., and Osley, M.A. (2000). Rad6-dependent ubiquitination of histone H2B in yeast. Science 287, 501-504.

Rodriguez, C.R., Cho, E.J., Keogh, M.C., Moore, C.L., Greenleaf, A.L., and Buratowski, S. (2000). Kin28, the TFIIH-associated carboxy-terminal domain kinase, facilitates the recruitment of mRNA processing machinery to RNA polymerase II. Mol. Cell Biol. 20, 104-112.

Romano, G. and Giordano, A. (2008). Role of the cyclin-dependent kinase 9-related pathway in mammalian gene expression and human diseases. Cell Cycle 7, 36643668 .

Rowley, J.D. (1998). The critical role of chromosome translocations in human leukemias. Annu. Rev. Genet. 32, 495-519.

Rozenblatt-Rosen, O., Nagaike, T., Francis, J.M., Kaneko, S., Glatt, K.A., Hughes, C.M., LaFramboise, T., Manley, J.L., and Meyerson, M. (2009). The tumor suppressor Cdc73 functionally associates with CPSF and CstF 3 ' mRNA processing factors. Proc. Natl. Acad. Sci. U. S. A 106, 755-760.

Rozovskaia, T., Feinstein, E., Mor, O., Foa, R., Blechman, J., Nakamura, T., Croce, C.M., Cimino, G., and Canaani, E. (2001). Upregulation of Meis1 and HoxA9 in 
acute lymphocytic leukemias with the $\mathrm{t}(4: 11)$ abnormality. Oncogene $20,874-$ 878.

Rual, J.F., Venkatesan, K., Hao, T., Hirozane-Kishikawa, T., Dricot, A., Li, N., Berriz, G.F., Gibbons, F.D., Dreze, M., Ayivi-Guedehoussou, N., Klitgord, N., Simon, C., Boxem, M., Milstein, S., Rosenberg, J., Goldberg, D.S., Zhang, L.V., Wong, S.L., Franklin, G., Li, S., Albala, J.S., Lim, J., Fraughton, C., Llamosas, E., Cevik, S., Bex, C., Lamesch, P., Sikorski, R.S., Vandenhaute, J., Zoghbi, H.Y., Smolyar, A., Bosak, S., Sequerra, R., Doucette-Stamm, L., Cusick, M.E., Hill, D.E., Roth, F.P., and Vidal, M. (2005). Towards a proteome-scale map of the human protein-protein interaction network. Nature 437, 1173-1178.

Saintigny, Y., Delacote, F., Vares, G., Petitot, F., Lambert, S., Averbeck, D., and Lopez, B.S. (2001). Characterization of homologous recombination induced by replication inhibition in mammalian cells. EMBO J. 20, 3861-3870.

Santos-Rosa, H., Schneider, R., Bannister, A.J., Sherriff, J., Bernstein, B.E., Emre, N.C., Schreiber, S.L., Mellor, J., and Kouzarides, T. (2002). Active genes are trimethylated at K4 of histone H3. Nature 419, 407-411.

Sarcevic, B., Mawson, A., Baker, R.T., and Sutherland, R.L. (2002). Regulation of the ubiquitin-conjugating enzyme hHR6A by CDK-mediated phosphorylation. EMBO J. 21, 2009-2018.

Saunders, A., Core, L.J., and Lis, J.T. (2006). Breaking barriers to transcription elongation. Nat. Rev. Mol. Cell Biol. 7, 557-567.

Schneider, J., Wood, A., Lee, J.S., Schuster, R., Dueker, J., Maguire, C., Swanson, S.K., Florens, L., Washburn, M.P., and Shilatifard, A. (2005). Molecular regulation of histone $\mathrm{H} 3$ trimethylation by COMPASS and the regulation of gene expression. Mol. Cell 19, 849-856.

Schneider, S., Pei, Y., Shuman, S., and Schwer, B. (2010). Separable functions of the fission yeast Spt5 CTD in capping enzyme binding and transcription elongation overlap with those of the RNA polymerase II CTD. Mol. Cell Biol.

Schroeder, S.C., Schwer, B., Shuman, S., and Bentley, D. (2000). Dynamic association of capping enzymes with transcribing RNA polymerase II. Genes Dev. 14, 2435 2440.

Schulman, B.A., Lindstrom, D.L., and Harlow, E. (1998). Substrate recruitment to cyclin-dependent kinase 2 by a multipurpose docking site on cyclin A. Proc. Natl. Acad. Sci. U. S. A 95, 10453-10458.

Schulze, A., Zerfass, K., Spitkovsky, D., Middendorp, S., Berges, J., Helin, K., JansenDurr, P., and Henglein, B. (1995). Cell cycle regulation of the cyclin A gene promoter is mediated by a variant E2F site. Proc. Natl. Acad. Sci. U. S. A 92, 11264-11268.

Schwartz, S., Meshorer, E., and Ast, G. (2009). Chromatin organization marks exonintron structure. Nat. Struct. Mol. Biol 16, 990-995. 
Shahbazian, M.D., Zhang, K., and Grunstein, M. (2005). Histone H2B ubiquitylation controls processive methylation but not monomethylation by Dot1 and Set1. Mol. Cell 19, 271-277.

Sheldon, K.E., Mauger, D.M., and Arndt, K.M. (2005). A requirement for the Saccharomyces cerevisiae Paf1 complex in snoRNA 3' end formation. Mol. Cell 20, 225-236.

Shema, E., Tirosh, I., Aylon, Y., Huang, J., Ye, C., Moskovits, N., Raver-Shapira, N., Minsky, N., Pirngruber, J., Tarcic, G., Hublarova, P., Moyal, L., Gana-Weisz, M., Shiloh, Y., Yarden, Y., Johnsen, S.A., Borivoj, V., Berger, S.L., and Oren, M. (2008). The histone H2B-specific ubiquitin ligase RNF20/hBRE1 acts as a putative tumor suppressor through selective regulation of gene expression. Genes Dev. 22, 2664-2.

Sherr, C.J. and Roberts, J.M. (1999). CDK inhibitors: positive and negative regulators of G1-phase progression. Genes Dev. 13, 1501-1512.

Sherr, C.J. and Roberts, J.M. (2004). Living with or without cyclins and cyclindependent kinases. Genes Dev. 18, 2699-2711.

Shi, X., Hong, T., Walter, K.L., Ewalt, M., Michishita, E., Hung, T., Carney, D., Pena, P., Lan, F., Kaadige, M.R., Lacoste, N., Cayrou, C., Davrazou, F., Saha, A., Cairns, B.R., Ayer, D.E., Kutateladze, T.G., Shi, Y., Cote, J., Chua, K.F., and Gozani, O. (2006). ING2 PHD domain links histone H3 lysine 4 methylation to active gene repression. Nature 442, 96-99.

Shi, Y., Di Giammartino, D.C., Taylor, D., Sarkeshik, A., Rice, W.J., Yates, J.R. III, Frank, J., and Manley, J.L. (2009). Molecular architecture of the human pre-mRNA 3' processing complex. Mol. Cell 33, 365-376.

Shilatifard, A. (2006). Chromatin modifications by methylation and ubiquitination: implications in the regulation of gene expression. Annu. Rev. Biochem. 75, 243269.

Shin, S., Rossow, K.L., Grande, J.P., and Janknecht, R. (2007). Involvement of RNA helicases p68 and p72 in colon cancer. Cancer Res. 67, 7572-7578.

Shirodkar, S., Ewen, M., Decaprio, J.A., Morgan, J., Livingston, D.M., and Chittenden, T. (1992). The transcription factor E2F interacts with the retinoblastoma product and a p107-cyclin A complex in a cell cycle-regulated manner. Cell 68, 157-166.

Shore, S.M., Byers, S.A., Maury, W., and Price, D.H. (2003). Identification of a novel isoform of Cdk9. Gene 307, 175-182.

Shuman, S. (2001). Structure, mechanism, and evolution of the mRNA capping apparatus. Prog. Nucleic Acid Res. Mol. Biol. 66, 1-40.

Simone, C., Bagella, L., Bellan, C., and Giordano, A. (2002a). Physical interaction between $\mathrm{pRb}$ and cdk9/cyclinT2 complex. Oncogene 21, 4158-4165. 
Simone, C., Stiegler, P., Bagella, L., Pucci, B., Bellan, C., De Falco, G., De Luca, A., Guanti, G., Puri, P.L., and Giordano, A. (2002b). Activation of MyoD-dependent transcription by cdk9/cyclin T2. Oncogene $21,4137-4148$.

Sims, R.J. III and Reinberg, D. (2006). Histone H3 Lys 4 methylation: caught in a bind? Genes Dev. 20, 2779-2786.

Sims, R.J. III and Reinberg, D. (2009). Processing the H3K36me3 signature. Nat. Genet. 41, 270-271.

Sims, R.J. III, Belotserkovskaya, R., and Reinberg, D. (2004). Elongation by RNA polymerase II: the short and long of it. Genes Dev. 18, 2437-2468.

Sims, R.J. III, Millhouse, S., Chen, C.F., Lewis, B.A., Erdjument-Bromage, H., Tempst, P., Manley, J.L., and Reinberg, D. (2007). Recognition of trimethylated histone H3 lysine 4 facilitates the recruitment of transcription postinitiation factors and premRNA splicing. Mol. Cell 28, 665-676.

Singer, M.S., Kahana, A., Wolf, A.J., Meisinger, L.L., Peterson, S.E., Goggin, C., Mahowald, M., and Gottschling, D.E. (1998). Identification of high-copy disruptors of telomeric silencing in Saccharomyces cerevisiae. Genetics 150, 613-632.

Sisodia, S.S., Sollner-Webb, B., and Cleveland, D.W. (1987). Specificity of RNA maturation pathways: RNAs transcribed by RNA polymerase III are not substrates for splicing or polyadenylation. Mol. Cell Biol. 7, 3602-3612.

Sittman, D.B., Graves, R.A., and Marzluff, W.F. (1983). Histone mRNA concentrations are regulated at the level of transcription and mRNA degradation. Proc. Natl. Acad. Sci. U. S. A. 7, 1849-1853.

Spies, N., Nielsen, C.B., Padgett, R.A., and Burge, C.B. (2009). Biased chromatin signatures around polyadenylation sites and exons. Mol. Cell 36, 245-254.

Steward, M.M., Lee, J.S., O'Donovan, A., Wyatt, M., Bernstein, B.E., and Shilatifard, A. (2006). Molecular regulation of H3K4 trimethylation by ASH2L, a shared subunit of MLL complexes. Nat. Struct. Mol. Biol 13, 852-854.

Strahl, B.D. and Allis, C.D. (2000). The language of covalent histone modifications. Nature 403, 41-45.

Strahl, B.D., Grant, P.A., Briggs, S.D., Sun, Z.W., Bone, J.R., Caldwell, J.A., Mollah, S., Cook, R.G., Shabanowitz, J., Hunt, D.F., and Allis, C.D. (2002). Set2 is a nucleosomal histone $\mathrm{H} 3$-selective methyltransferase that mediates transcriptional repression. Mol. Cell Biol. 22, 1298-1306.

Su, C., Gao, G., Schneider, S., Helt, C., Weiss, C., O'Reilly, M.A., Bohmann, D., and Zhao, J. (2004). DNA damage induces downregulation of histone gene expression through the G1 checkpoint pathway. EMBO J. 23, 1133-1143.

Suganuma, T. and Workman, J.L. (2008). Crosstalk among histone modifications. Cell $135,604-607$. 
Sullivan, E., Santiago, C., Parker, E.D., Dominski, Z., Yang, X., Lanzotti, D.J., Ingledue, T.C., Marzluff, W.F., and Duronio, R.J. (2001). Drosophila stem loop binding protein coordinates accumulation of mature histone mRNA with cell cycle progression. Genes Dev. 15, 173-187.

Sullivan, K.D., Mullen, T.E., Marzluff, W.F., and Wagner, E.J. (2009). Knockdown of SLBP results in nuclear retention of histone mRNA. RNA. 14, 459-472.

Sun, X.J., Wei, J., Wu, X.Y., Hu, M., Wang, L., Wang, H.H., Zhang, Q.H., Chen, S.J., Huang, Q.H., and Chen, Z. (2005). Identification and characterization of a novel human histone H3 lysine 36-specific methyltransferase. J. Biol Chem. 280, 3526135271 .

Sun, Z.W. and Allis, C.D. (2002). Ubiquitination of histone H2B regulates H3 methylation and gene silencing in yeast. Nature 418, 104-108.

Svejstrup, J.Q., Vichi, P., and Egly, J.M. (1996). The multiple roles of transcription/repair factor TFIIH. Trends Biochem. Sci. 21, 346-350.

Takagaki, Y. and Manley, J.L. (2000). Complex protein interactions within the human polyadenylation machinery identify a novel component. Mol. Cell Biol. 20, 15151525.

Takahashi, Y., Rayman, J.B., and Dynlacht, B.D. (2000). Analysis of promoter binding by the E2F and $\mathrm{pRB}$ families in vivo: distinct E2F proteins mediate activation and repression. Genes Dev. 14, 804-816.

Tenney, K. and Shilatifard, A. (2005). A COMPASS in the voyage of defining the role of trithorax/MLL-containing complexes: linking leukemogensis to covalent modifications of chromatin. J. Cell Biochem. 95, 429-436.

Thoma, F., Koller, T., and Klug, A. (1979). Involvement of histone H1 in the organization of the nucleosome and of the salt-dependent superstructures of chromatin. J. Cell Biol. 83, 403-427.

Turner, B.M. (2007). Defining an epigenetic code. Nat. Cell Biol. 9, 2-6.

Tyagi, S., Chabes, A.L., Wysocka, J., and Herr, W. (2007). E2F activation of S phase promoters via association with HCF-1 and the MLL family of histone H3K4 methyltransferases. Mol. Cell 27, 107-119.

Vairo, G., Livingston, D.M., and Ginsberg, D. (1995). Functional interaction between E2F-4 and p130: evidence for distinct mechanisms underlying growth suppression by different retinoblastoma protein family members. Genes Dev. 9, 869-881.

Vasserot, A.P., Schaufele, F.J., and Birnstiel, M.L. (1989). Conserved terminal hairpin sequences of histone mRNA precursors are not involved in duplex formation with the U7 RNA but act as a target site for a distinct processing factor. Proc. Natl. Acad. Sci. U. S. A 86, 4345-4349.

Vassilev, L.T. (2007). MDM2 inhibitors for cancer therapy. Trends Mol. Med. 13, 2331. 
Vassilev, L.T., Vu, B.T., Graves, B., Carvajal, D., Podlaski, F., Filipovic, Z., Kong, N., Kammlott, U., Lukacs, C., Klein, C., Fotouhi, N., and Liu, E.A. (2004). In vivo activation of the p53 pathway by small-molecule antagonists of MDM2. Science $303,844-848$.

Vazquez, A., Bond, E.E., Levine, A.J., and Bond, G.L. (2008). The genetics of the p53 pathway, apoptosis and cancer therapy. Nat. Rev. Drug Discov. 7, 979-987.

Vermeulen, K., Van Bockstaele, D.R., and Berneman, Z.N. (2003). The cell cycle: a review of regulation, deregulation and therapeutic targets in cancer. Cell Prolif. 36, 131-149.

Vinciguerra, P. and Stutz, F. (2004). mRNA export: an assembly line from genes to nuclear pores. Curr. Opin. Cell Biol. 16, 285-292.

Wada, T., Orphanides, G., Hasegawa, J., Kim, D.K., Shima, D., Yamaguchi, Y., Fukuda, A., Hisatake, K., Oh, S., Reinberg, D., and Handa, H. (2000). FACT relieves DSIF/NELF-mediated inhibition of transcriptional elongation and reveals functional differences between P-TEFb and TFIIH. Mol. Cell 5, 1067-1072.

Wada, T., Takagi, T., Yamaguchi, Y., Ferdous, A., Imai, T., Hirose, S., Sugimoto, S., Yano, K., Hartzog, G.A., Winston, F., Buratowski, S., and Handa, H. (1998a). DSIF, a novel transcription elongation factor that regulates RNA polymerase II processivity, is composed of human Spt4 and Spt5 homologs. Genes Dev. 12, 343356.

Wada, T., Takagi, T., Yamaguchi, Y., Watanabe, D., and Handa, H. (1998b). Evidence that $\mathrm{P}-\mathrm{TEFb}$ alleviates the negative effect of DSIF on RNA polymerase IIdependent transcription in vitro. EMBO J. 17, 7395-7403.

Wagner, E.J. and Marzluff, W.F. (2006). ZFP100, a component of the active U7 snRNP limiting for histone pre-mRNA processing, is required for entry into $\mathrm{S}$ phase. Mol. Cell Biol 26, 6702-6712.

Wagner, E.J., Berkow, A., and Marzluff, W.F. (2005). Expression of an RNAi-resistant SLBP restores proper S-phase progression. Biochem. Soc. Trans. 33, 471-473.

Wagner, E.J., Burch, B.D., Godfrey, A.C., Salzler, H.R., Duronio, R.J., and Marzluff, W.F. (2007). A genome-wide RNA interference screen reveals that variant histones are necessary for replication-dependent histone pre-mRNA processing. Mol. Cell 28, 692-699.

Wang, H., Zhai, L., Xu, J., Joo, H.Y., Jackson, S., Erdjument-Bromage, H., Tempst, P., Xiong, Y., and Zhang, Y. (2006). Histone H3 and H4 ubiquitylation by the CUL4DDB-ROC1 ubiquitin ligase facilitates cellular response to DNA damage. Mol. Cell 22, 383-394.

Wang, Z.F., Whitfield, M.L., Ingledue, T.C., III, Dominski, Z., and Marzluff, W.F. (1996). The protein that binds the 3' end of histone mRNA: a novel RNA-binding protein required for histone pre-mRNA processing. Genes Dev. 10, 3028-3040.

Weake, V.M. and Workman, J.L. (2008). Histone ubiquitination: triggering gene activity. Mol. Cell 29, 653-663. 
Wei, Y., Jin, J., and Harper, J.W. (2003). The cyclin E/Cdk2 substrate and Cajal body component p220(NPAT) activates histone transcription through a novel LisH-like domain. Mol. Cell Biol 23, 3669-3680.

Weinberg, R.A. (1995). The retinoblastoma protein and cell cycle control. Cell 81, 323330.

Weiner, A.M. (2005). E Pluribus Unum: 3' end formation of polyadenylated mRNAs, histone mRNAs, and U snRNAs. Mol. Cell 20, 168-170.

Wells, D. and Kedes, L. (1985). Structure of a human histone cDNA: evidence that basally expressed histone genes have intervening sequences and encode polyadenylylated mRNAs. Proc. Natl. Acad. Sci. U. S. A 82, 2834-2838.

Wen, Y. and Shatkin, A.J. (1999). Transcription elongation factor hSPT5 stimulates mRNA capping. Genes Dev. 13, 1774-1779.

Wetterberg, I., Zhao, J., Masich, S., Wieslander, L., and Skoglund, U. (2001). In situ transcription and splicing in the Balbiani ring 3 gene. EMBO J. 20, 2564-2574.

Whitfield, M.L., Kaygun, H., Erkmann, J.A., Townley-Tilson, W.H., Dominski, Z., and Marzluff, W.F. (2004). SLBP is associated with histone mRNA on polyribosomes as a component of the histone mRNP. Nucleic Acids Res. 32, 4833-4842.

Wickens, M., Anderson, P., and Jackson, R.J. (1997). Life and death in the cytoplasm: messages from the 3' end. Curr. Opin. Genet. Dev. 7, 220-232.

Wickliffe, K., Williamson, A., Jin, L., and Rape, M. (2009). The multiple layers of ubiquitin-dependent cell cycle control. Chem. Rev. 109, 1537-1548.

Williams, A.S. and Marzluff, W.F. (1995). The sequence of the stem and flanking sequences at the 3 ' end of histone mRNA are critical determinants for the binding of the stem-loop binding protein. Nucleic Acids Res. 23, 654-662.

Wilson, A.C. (2007). Setting the stage for S phase. Mol. Cell 27, 176-177.

Wilusz, C.J., Wormington, M., and Peltz, S.W. (2001). The cap-to-tail guide to mRNA turnover. Nat. Rev. Mol. Cell Biol. 2, 237-246.

Wood, A. and Shilatifard, A. (2006). Bur1/Bur2 and the Ctk complex in yeast: the split personality of mammalian P-TEFb. Cell Cycle 5, 1066-1068.

Wood, A., Krogan, N.J., Dover, J., Schneider, J., Heidt, J., Boateng, M.A., Dean, K., Golshani, A., Zhang, Y., Greenblatt, J.F., Johnston, M., and Shilatifard, A. (2003a). Bre1, an E3 ubiquitin ligase required for recruitment and substrate selection of Rad6 at a promoter. Mol. Cell 11, 267-274.

Wood, A., Schneider, J., Dover, J., Johnston, M., and Shilatifard, A. (2003b). The Paf1 complex is essential for histone monoubiquitination by the Rad6-Bre1 complex, which signals for histone methylation by COMPASS and Dot1p. J. Biol Chem. 278, 34739-34742. 
Wood, A., Schneider, J., Dover, J., Johnston, M., and Shilatifard, A. (2005). The Bur1/Bur2 complex is required for histone H2B monoubiquitination by Rad6/Bre1 and histone methylation by COMPASS. Mol. Cell 20, 589-599.

Wood, A., Shukla, A., Schneider, J., Lee, J.S., Stanton, J.D., Dzuiba, T., Swanson, S.K., Florens, L., Washburn, M.P., Wyrick, J., Bhaumik, S.R., and Shilatifard, A. (2007). Ctk complex-mediated regulation of histone methylation by COMPASS. Mol. Cell Biol 27, 709-720.

Wu, C.H. and Gall, J.G. (1993). U7 small nuclear RNA in C snurposomes of the Xenopus germinal vesicle. Proc. Natl. Acad. Sci. U. S. A 90, 6257-6259.

Wu, J., Huen, M.S., Lu, L.Y., Ye, L., Dou, Y., Ljungman, M., Chen, J., and Yu, X. (2009). Histone ubiquitination associates with BRCA1-dependent DNA damage response. Mol. Cell Biol 29, 849-860.

Wu, S.Y. and Chiang, C.M. (2007). The double bromodomain-containing chromatin adaptor Brd4 and transcriptional regulation. J. Biol. Chem. 282, 13141-13145.

Wyce, A., Xiao, T., Whelan, K.A., Kosman, C., Walter, W., Eick, D., Hughes, T.R., Krogan, N.J., Strahl, B.D., and Berger, S.L. (2007). H2B ubiquitylation acts as a barrier to Ctk1 nucleosomal recruitment prior to removal by Ubp8 within a SAGArelated complex. Mol. Cell 27, 275-288.

Xiao, T., Hall, H., Kizer, K.O., Shibata, Y., Hall, M.C., Borchers, C.H., and Strahl, B.D. (2003). Phosphorylation of RNA polymerase II CTD regulates H3 methylation in yeast. Genes Dev. 17, 654-663.

Xiao, T., Kao, C.F., Krogan, N.J., Sun, Z.W., Greenblatt, J.F., Osley, M.A., and Strahl, B.D. (2005). Histone H2B ubiquitylation is associated with elongating RNA polymerase II. Mol. Cell Biol. 25, 637-651.

Xiao, T., Shibata, Y., Rao, B., Laribee, R.N., O'Rourke, R., Buck, M.J., Greenblatt, J.F., Krogan, N.J., Lieb, J.D., and Strahl, B.D. (2007). The RNA polymerase II kinase Ctk1 regulates positioning of a 5' histone methylation boundary along genes. Mol. Cell Biol 27, 721-731.

Yamada, T., Yamaguchi, Y., Inukai, N., Okamoto, S., Mura, T., and Handa, H. (2006). $\mathrm{P}$-TEFb-mediated phosphorylation of $\mathrm{hSpt} 5 \mathrm{C}$-terminal repeats is critical for processive transcription elongation. Mol. Cell 21, 227-237.

Yamaguchi, Y., Inukai, N., Narita, T., Wada, T., and Handa, H. (2002). Evidence that negative elongation factor represses transcription elongation through binding to a DRB sensitivity-inducing factor/RNA polymerase II complex and RNA. Mol. Cell Biol 22, 2918-2927.

Yamaguchi, Y., Takagi, T., Wada, T., Yano, K., Furuya, A., Sugimoto, S., Hasegawa, J., and Handa, H. (1999). NELF, a multisubunit complex containing RD, cooperates with DSIF to repress RNA polymerase II elongation. Cell 97, 41-51.

Yan, B., Yang, X., Lee, T.L., Friedman, J., Tang, J., Van Waes, C., and Chen, Z. (2007). Genome-wide identification of novel expression signatures reveal distinct patterns and prevalence of binding motifs for $\mathrm{p} 53$, nuclear factor-kappaB and other 
signal transcription factors in head and neck squamous cell carcinoma. Genome Biol 8, R78.

Yang, X.C., Burch, B.D., Yan, Y., Marzluff, W.F., and Dominski, Z. (2009). FLASH, a proapoptotic protein involved in activation of caspase-8, is essential for 3 ' end processing of histone pre-mRNAs. Mol. Cell 36, 267-278.

Yang, Z., Yik, J.H., Chen, R., He, N., Jang, M.K., Ozato, K., and Zhou, Q. (2005). Recruitment of $\mathrm{P}-\mathrm{TEFb}$ for stimulation of transcriptional elongation by the bromodomain protein Brd4. Mol. Cell 19, 535-545.

Yang, Z., Zhu, Q., Luo, K., and Zhou, Q. (2001). The 7SK small nuclear RNA inhibits the CDK9/cyclin T1 kinase to control transcription. Nature 414, 317-322.

Ye, X., Wei, Y., Nalepa, G., and Harper, J.W. (2003). The cyclin E/Cdk2 substrate p220(NPAT) is required for S-phase entry, histone gene expression, and Cajal body maintenance in human somatic cells. Mol. Cell Biol 23, 8586-8600.

Yeo, M., Lee, S.K., Lee, B., Ruiz, E.C., Pfaff, S.L., and Gill, G.N. (2005). Small CTD phosphatases function in silencing neuronal gene expression. Science 307, 596600 .

Yeo, M., Lin, P.S., Dahmus, M.E., and Gill, G.N. (2003). A novel RNA polymerase II C-terminal domain phosphatase that preferentially dephosphorylates serine 5. J. Biol Chem. 278, 26078-26085.

Yik, J.H., Chen, R., Nishimura, R., Jennings, J.L., Link, A.J., and Zhou, Q. (2003). Inhibition of P-TEFb (CDK9/Cyclin $\mathrm{T}$ ) kinase and RNA polymerase II transcription by the coordinated actions of HEXIM1 and 7SK snRNA. Mol. Cell 12, 971-982.

Yoh, S.M., Cho, H., Pickle, L., Evans, R.M., and Jones, K.A. (2007). The Spt6 SH2 domain binds Ser2-P RNAPII to direct Iws1-dependent mRNA splicing and export. Genes Dev. 21, 160-174.

Zeitlinger, J., Stark, A., Kellis, M., Hong, J.W., Nechaev, S., Adelman, K., Levine, M., and Young, R.A. (2007). RNA polymerase stalling at developmental control genes in the Drosophila melanogaster embryo. Nat. Genet. 39, 1512-1516.

Zhang, X.Y., Varthi, M., Sykes, S.M., Phillips, C., Warzecha, C., Zhu, W., Wyce, A., Thorne, A.W., Berger, S.L., and McMahon, S.B. (2008). The putative cancer stem cell marker USP22 is a subunit of the human SAGA complex required for activated transcription and cell-cycle progression. Mol. Cell 29, 102-111.

Zhang, Y. (2003). Transcriptional regulation by histone ubiquitination and deubiquitination. Genes Dev. 17, 2733-2740.

Zhang, Y. (2006). It takes a PHD to interpret histone methylation. Nat. Struct. Mol. Biol. 13, 572-574.

Zhao, H., Langerod, A., Ji, Y., Nowels, K.W., Nesland, J.M., Tibshirani, R., Bukholm, I.K., Karesen, R., Botstein, D., Borresen-Dale, A.L., and Jeffrey, S.S. (2004). 
Different gene expression patterns in invasive lobular and ductal carcinomas of the breast. Mol. Biol Cell 15, 2523-2536.

Zhao, J. (2004). Coordination of DNA synthesis and histone gene expression during normal cell cycle progression and after DNA damage. Cell Cycle 3, 695-697.

Zhao, J., Dynlacht, B., Imai, T., Hori, T., and Harlow, E. (1998). Expression of NPAT, a novel substrate of cyclin E-CDK2, promotes S-phase entry. Genes Dev. 12, 456461.

Zhao, J., Kennedy, B.K., Lawrence, B.D., Barbie, D.A., Matera, A.G., Fletcher, J.A., and Harlow, E. (2000). NPAT links cyclin E-Cdk2 to the regulation of replicationdependent histone gene transcription. Genes Dev. 14, 2283-2297.

Zheng, L., Dominski, Z., Yang, X.C., Elms, P., Raska, C.S., Borchers, C.H., and Marzluff, W.F. (2003a). Phosphorylation of stem-loop binding protein (SLBP) on two threonines triggers degradation of SLBP, the sole cell cycle-regulated factor required for regulation of histone mRNA processing, at the end of S phase. Mol. Cell Biol. 23, 1590-1601.

Zheng, L., Roeder, R.G., and Luo, Y. (2003b). S phase activation of the histone H2B promoter by OCA-S, a coactivator complex that contains GAPDH as a key component. Cell 114, 255-266.

Zhou, M., Deng, L., Lacoste, V., Park, H.U., Pumfery, A., Kashanchi, F., Brady, J.N., and Kumar, A. (2004). Coordination of transcription factor phosphorylation and histone methylation by the P-TEFb kinase during human immunodeficiency virus type 1 transcription. J. Virol. 78, 13522-13533.

Zhu, B., Mandal, S.S., Pham, A.D., Zheng, Y., Erdjument-Bromage, H., Batra, S.K., Tempst, P., and Reinberg, D. (2005a). The human PAF complex coordinates transcription with events downstream of RNA synthesis. Genes Dev. 19, 16681673.

Zhu, B., Zheng, Y., Pham, A.D., Mandal, S.S., Erdjument-Bromage, H., Tempst, P., and Reinberg, D. (2005b). Monoubiquitination of human histone H2B: the factors involved and their roles in HOX gene regulation. Mol. Cell 20,601-611.

Zhu, L., Harlow, E., and Dynlacht, B.D. (1995). p107 uses a p21CIP1-related domain to bind cyclin/cdk2 and regulate interactions with E2F. Genes Dev. 9, 1740-1752.

zur Hausen, H. and de Villiers, E.M. (1994). Human papillomaviruses. Annu. Rev. Microbiol. 48, 427-447. 


\section{Acknowledgements}

First of all, I would like to thank my supervisor Prof. Dr. Steven A. Johnsen for his intense support and guidance throughout the last three years. I am thankful for his excellent scientific supervision, his inspirational and encouraging ideas and all his help. Moreover, I want to thank him for the friendly atmosphere he created in our group and for being available at all times.

I am grateful to Prof. Dr. Matthias Dobbelstein for giving me the opportunity to work in his department of Molecular Oncology and for interesting and helpful discussions and suggestions.

I thank my PhD committee members Prof. Dr. Heidi Hahn and Prof. Dr. Holger Reichardt for their "outside" perspective to my work, their interest in my project, their time and support. In addition, I would like to thank Prof. Dr. Heidi Hahn for being my second referee.

I want to thank Prof. Dr. Reinhardt Lührmann for giving me the opportunity to perform some experiments at his department of Cellular Biochemistry at the MPI for Biophysical Chemistry and Dr. Ira Lemm for her help and guidance during these experiments.

I thank all the lab members of the Dobbelstein, the Moll and in particular the Johnsen group for the nice and friendly atmosphere in the lab and for helping me in one way or the other to carry out my work successfully. My thanks go especially to Antje and Cathrin for their technical assistance and support and to Isa, Lisa, Richard, Tanja, Theresa and Ulli for being excellent colleagues and friends.

Finally, my parents, my sister and my life partner Stephan deserve special mention. I want to thank them for their continuous support, their constant encouragement, their understanding and help and for being there for me at every step of the way. 


\section{Curriculum Vitae}

\section{Personal data}

$\begin{array}{ll}\text { Name: } & \text { Judith Pirngruber } \\ \text { Date of birth: } & \text { June } 3^{\text {rd }}, 1982 \\ \text { Place of birth: } & \text { Oldenburg, Germany } \\ \text { Nationality: } & \text { German and Austrian }\end{array}$

\section{Education}

since $04 / 2007$

Doctoral thesis

Georg-August-Universität Göttingen

Thesis: "Epigenetic Regulation of Replication-Dependent Histone mRNA 3' End Processing”

10/2002 - 02/2007 Diploma studies in Biology

Carl-von-Ossietzky Universität Oldenburg

Thesis: "Functional Morphology of the retina of a Proteinkinase

$C \alpha$-deficient mouse line"

08/1994 - 06/2001 Gymnasium Bad Zwischenahn/Edewecht

Abitur

Major subjects: Biology and French

08/1992 - 07/1994 Orientierungsstufe Friedrichsfehn

08/1988 - 06/1992 Grundschule Friedrichsfehn (Elementary School)

\section{Awards}

since $10 / 2008$

Excellent individual stipend of the "Göttingen Graduate School for Neurosciences and Molecular Biosciences (GGNB)"

\section{Additional publications}

Ecke, I., Petry, F., Rosenberger, A., Tauber, S., Mönkemeyer, S., Hess,I., Dullin, C., Kimmina, S., Pirngruber, J., Johnsen, S.A., Uhmann, A., Nitzki, F., Wojnowski, L., Schulz-Schaeffer, W., Witt, O., and Hahn, H. (2009). Antitumor effects of a combined 5-aza-2'deoxycytidine and valproic acid treatment on rhabdomyosarcoma and medulloblastoma in Ptch mutant mice. Cancer Res. 69, 887-895.

Shema, E., Tirosh, I., Aylon, Y., Huang, J., Ye, C., Moskovits, N., Raver-Shapira, N., Minsky, N., Pirngruber, J., Tarcic, G., Hublarova, P., Moyal, L., Gana-Weisz, M., Shiloh, Y., Yarden, Y., Johnsen, S.A., Borivoj, V., Berger, S.L., and Oren, M. (2008). The histone H2B-specific ubiquitin ligase RNF20/hBRE1 acts as a putative tumor suppressor through selective regulation of gene expression. Genes Dev. 22, 2664-2. 